\title{
Convergent Synthesis of a Complex Oxime Library Using Chemical Domain Shuffling
}

\author{
Shun Su, Dayle E. Acquilano, Jeevanandam Arumugasamy, Aaron B. Beeler, Erin L. Eastwood, \\ Joshua R. Giguere, Ping Lan, Xiaoguang Lei, Geanna K. Min, Adam R. Yeager, Ya Zhou, James S. \\ Panek, John K. Snyder, Scott E. Schaus, ${ }^{\star}$ and John A. Porco, Jr. * \\ Center for Chemical Methodology and Library Development at Boston University (CMLD-BU) \\ Department of Chemistry, Life and Science Building, Boston University, \\ 24 Cummington Street, Boston, Massachusetts 02215
}

\section{Supporting Information}

\section{General Experimental Procedures}

${ }^{1} \mathrm{H}$ NMR spectra were recorded on a Varian Mercury $400 \mathrm{MHz}$ spectrometer at ambient temperature in $\mathrm{CDCl}_{3}$ unless otherwise stated. ${ }^{13} \mathrm{C} \mathrm{NMR}$ spectra were recorded at $100 \mathrm{MHz}$ spectrometer (unless otherwise stated) at ambient temperature. Chemical shifts are reported in parts per million relative to chloroform $\left({ }^{1} \mathrm{H}, \delta 7.24 ;{ }^{13} \mathrm{C}, \delta 77.23\right)$. Data for ${ }^{1} \mathrm{H} \mathrm{NMR}$ are reported as follows: chemical shift, integration, multiplicity $(\operatorname{app}=$ apparent, par obsc $=$ partially obscure, ovrlp $=$ overlapping, $\mathrm{s}=$ singlet, $\mathrm{d}=$ doublet, $\mathrm{t}=$ triplet, $\mathrm{q}=$ quartet, $\mathrm{m}=$ multiplet) and coupling constants. All ${ }^{13} \mathrm{C}$ NMR spectra were recorded with complete proton decoupling. Infrared spectra were recorded on a Nicolet Nexus 470 FTIR + Continu $\mu \mathrm{M}$ microscope. Optical rotations were recorded on an AUTOPOL III digital polarimeter at $589 \mathrm{~nm}$, and are recorded as $[\alpha]_{\mathrm{D}}$ (concentration in grams/100 $\mathrm{mL}$ solvent). HPLC-ELSD-MS analysis was performed on a Waters HPLC-MS system equipped with Waters 600 HPLC pump, Waters 2996 photodiode array detector, Micromass ZQ Quadrupole Mass Spectrometer, and Sedere Sedex 75 ELS detector (XTerra ${ }^{\circledR} \mathrm{RP}_{8}, 5 \mu \mathrm{m}, 4.6 \times 30 \mathrm{~mm}$; Atlantis ${ }^{\mathrm{TM}} \mathrm{dC}_{18}, 5 \mu \mathrm{m}, 4.6 \times 50 \mathrm{~mm}$ ). Preparative HPLC was performed on an XTerra ${ }^{\circledR}$ Prep $\mathrm{RP}_{18}$ column $(5 \mu \mathrm{m}, 19 \times 50 \mathrm{~mm})$. Analytical thin layer chromatography was performed on $0.25 \mathrm{~mm}$ silica gel $60-\mathrm{F}$ plates. Flash chromatography was performed on a CombiFlash ${ }^{\circledR}$ Sq16X system (Teledyne ISCO) using normal phase RediSep ${ }^{\text {TM }}$ flash columns. For library synthesis, the AutoChem MiniMapper ${ }^{\mathrm{TM}}$ was emploied for liquid handling operations. Microwave-assisted reactions were carried out using a CEM Discover Explorer system (CEM Corporation, Matthews, NC.). Reactions involving solid-phase reagents were performed using a Quest 210 (Argonaut, Redwood City, CA.). Sample evaporation was performed using GeneVac ${ }^{\circledR}$ EZ-2 and HT-4 evaporator systems. Final products were weighted using Balance Automator ${ }^{\mathrm{TM}}$ (AutoChem). Ozonolysis was conducted using a WELSBACH ozone generator. Supported liquid-liquid extraction performed using 5.0 mL ISOLUTE ${ }^{\circledR}$ HM-N Supported Liquid Extraction columns (part \# 800-0100CM, Argonaut). Solid-supported methyl isatoic anhydride resin (PL-MIA, part \# 3405-3679, 2.57 $\mathrm{mmol} / \mathrm{g}$ ) was obtained from Polymer Laboratories. PS-Diethylenetriamine resin was prepared according to the literature procedure. ${ }^{1}$ All other reagents were used as supplied by Sigma-Aldrich, Lancaster Synthesis, Strem, and Argonaut Technologies. Methylene chloride, tetrahydrofuran, methanol, benzene were purified by passing through two packed columns of neutral alumina (Glass Contour, Irvine, CA).

Library enumeration was carried out using ChemDraw for Excel (8.0 April 15, 2003). Calculations of cLogP and total polar surface area (TPSA) were performed using the Molinspiration mitools toolkit software package (http://www.molinspiration.com). Arthur ${ }^{\mathrm{TM}}$ Suite Reaction Planner (Synthematix, Inc.) was used for experimental procedure planning.

${ }^{1}$ Parlow, J. J.; Mischke, D. A.; Woodard, S. S. J. Org. Chem. 1997, 62, 5908-5919. 


\section{Monomer Syntheses}

\subsection{Synthesis of carbonyl domain monomers.}

Scheme 1. Synthesis of 18.

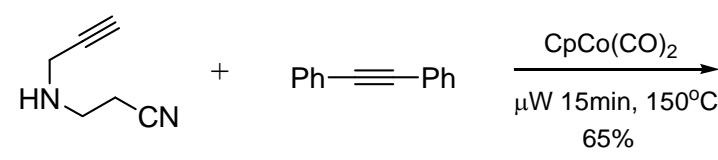

34

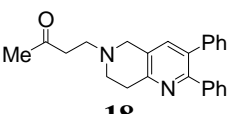

18

4-(6,7-Dimethyl-3,4-dihydro-2H-[1,5]naphthyridin-1-yl)-butan-2-one (18): To a 10 $\mathrm{mL}$ high pressure tube under an atmosphere of nitrogen was added $34(0.50 \mathrm{~g}, 4.6$ mmol), diphenylacetylene (35) (2.74 g, $15.4 \mathrm{mmol}), \mathrm{CpCo}(\mathrm{CO})_{2}(200 \mathrm{mg}, 1.1 \mathrm{~mol})$ and chlorobenzene $(20 \mathrm{~mL})$. The reaction mixture was subjected to microwave irradiation for $15 \mathrm{~min}$ at $150{ }^{\circ} \mathrm{C}(150 \mathrm{~W})$, concentrated, and chromatographed on silica gel (EtOAc:MeOH=3:1) to provide 36 as a yellow solid. m.p. 156.0-158.0 ${ }^{\circ} \mathrm{C} ;{ }^{1} \mathrm{H}$ NMR $\left(400 \mathrm{MHz}, \mathrm{CDCl}_{3}\right) \delta 7.38(\mathrm{~s}, 1 \mathrm{H}), 7.31(\mathrm{~m}, 2 \mathrm{H}), 7.23$ $(\mathrm{m}, 6 \mathrm{H}), 7.13(\mathrm{~m}, 2 \mathrm{H}), 4.14(\mathrm{~s}, 1 \mathrm{H}), 3.33(\mathrm{t}, 2 \mathrm{H}, J=0.8 \mathrm{~Hz}), 3.10(\mathrm{t}, 2 \mathrm{H}, J=0.8 \mathrm{~Hz}) ;{ }^{13} \mathrm{C} \mathrm{NMR}(100$ $\left.\mathrm{MHz}_{2} \mathrm{CDCl}_{3}\right) \delta 155.4,153.8,140.4,140.0,136.7,134.0,130.1,129.8,129.3,128.5,128.2,127.9$, 127.3, 47.1, 44.0, 32.4; IR (thin film) vmax 3256, 3056, 2927, 1601, 1578, 1550, 1427, 1450, 1397, 796, 768, 738, $686 \mathrm{~cm}^{-1}$; LRMS [M+H] $]^{+}$calculated for $\mathrm{C}_{20} \mathrm{H}_{18} \mathrm{~N}_{2}$ : 287.1548, found: 286.8. 5,6,7,8tetrahydro-2,3-diphenyl-1,6-naphthyridine (36) (146 mg, $0.51 \mathrm{mmol})$ in $\mathrm{MeCN}(5 \mathrm{~mL})$, methyl vinyl ketone $(0.083 \mathrm{~mL}, 1.02 \mathrm{mmol})$ and ytterbium (III) triflate $(31.6 \mathrm{mg}, 0.051 \mathrm{mmol})$ were added to a 1 neck round bottom flask under an atmosphere of nitrogen, and the mixture was heated at reflux for 20 $\mathrm{h}$. The reaction mixture was then passed through a small silica gel plug and concentrated. The residue was chromatographed on silica gel (EtOAc:hexanes $=8: 1)$ to afford 18 as a pale yellow oil (136 $\mathrm{mg}$, $0.383 \mathrm{mmol}, 75 \%) .{ }^{1} \mathrm{H} \mathrm{NMR}\left(400 \mathrm{MHz}, \mathrm{CDCl}_{3}\right) \delta 7.36(\mathrm{~s}, 1 \mathrm{H}), 7.29(\mathrm{~m}, 2 \mathrm{H}), 7.23(\mathrm{~m}, 6 \mathrm{H}), 7.12(\mathrm{~m}$, 2H), $3.72(\mathrm{~s}, 2 \mathrm{H}), 3.14(\mathrm{t}, 2 \mathrm{H}, J=6.8 \mathrm{~Hz}), 2.90(\mathrm{~m}, 2 \mathrm{H}), 2.67(\mathrm{t}, 2 \mathrm{H}, J=6.8 \mathrm{~Hz}), 2.21(\mathrm{~s}, 3 \mathrm{H}) ;{ }^{13} \mathrm{C}$ NMR $\left(100 \mathrm{MHz}, \mathrm{CDCl}_{3}\right) \delta 207.9,155.4,153.5,140.3,140.0,136.8,133.7,129.9,129.7,128.3,128.0$, $127.7,127.1,60.5,55.0,52.4,51.1,41.8,32.3,30.4$; IR (thin film) vmax 3058, 2930, 2821, 1712 , $1649,1601,1554,1452,1429,1376,1259,1171,1113,1036,918 \mathrm{~cm}^{-1}$; CIHRMS [M] $]^{+}$calculated for $\mathrm{C}_{24} \mathrm{H}_{24} \mathrm{~N}_{2} \mathrm{O}: 356.1889$, found: 356.1900 .

Scheme 2. Synthesis of 19.
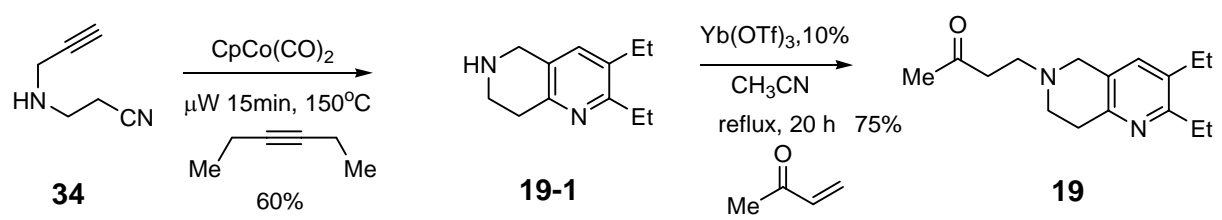

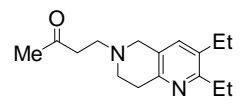

19

4-(2,3-Diethyl-7,8-dihydro-1,6-naphthyridin-6(5H)-yl)butan-2-one (19): To a 10 $\mathrm{mL}$ high pressure tube under an atmosphere of nitrogen, 34 (0.50 g, $4.6 \mathrm{mmol})$, hex-3yne $(1.27 \mathrm{~g}, 15.4 \mathrm{mmol})$ and $\mathrm{CpCo}(\mathrm{CO})_{2}(200 \mathrm{mg}, 1.1 \mathrm{~mol})$ were mixed with chlorobenzene $(20 \mathrm{~mL}){ }^{2}$ The reaction was subjected to microwave irradiation for 15

\footnotetext{
${ }^{2}$ For a review of construction of pyridine rings by metal-mediated [ $\left.2+2+2\right]$ cycloaddition, see: Varela, J. A.; Saa, C. Chem. Rev. 2003, 103, 3787-3802.
} 
min at $150{ }^{\circ} \mathrm{C}(150 \mathrm{~W})$. The mixture was cooled to rt, concentrated, and chromatographed on silica gel (EtOAc: $\mathrm{MeOH}=3: 1$ ) to provide 19-1 as a yellow oil. 2,3-diethyl-5,6,7,8-tetrahydro-1,6-naphthyridine (19-1) $(240 \mathrm{mg}, 1.27 \mathrm{mmol})$, methyl vinyl ketone $(0.232 \mathrm{~mL}, 2.8 \mathrm{mmol})$, and ytterbium(III) triflate $^{3}$ (90 mg, $0.146 \mathrm{mmol}$ ) were added into a 1-neck round-bottom flask containing $5 \mathrm{~mL}$ of MeCN under an atmosphere of nitrogen and the mixture was heated to reflux for 20 hours. The mixture was filtered, concentrated, and chromatographed on silica gel (EtOAc:MeOH=3:1) to yield $\mathbf{1 9}$ as a yellow oil (265 $\mathrm{mg}, 1.02 \mathrm{mmol}, 75 \%) .{ }^{1} \mathrm{H}$ NMR $\left(400 \mathrm{MHz}, \mathrm{CDCl}_{3}\right) \delta 6.99(\mathrm{~s}, 1 \mathrm{H}), 3.50(\mathrm{~s}, 2 \mathrm{H}), 2.88(\mathrm{t}, 2 \mathrm{H}, J=6.8$ $\mathrm{Hz}), 2.74(\mathrm{~m}, 4 \mathrm{H}), 2.66(\mathrm{~m}, 4 \mathrm{H}), 2.52(\mathrm{q}, 2 \mathrm{H}, J=7.0 \mathrm{~Hz}), 2.11(\mathrm{~s}, 3 \mathrm{H}), 1.14(\mathrm{t}, 3 \mathrm{H}, J=7.0 \mathrm{~Hz}) ; 1.11(\mathrm{t}$, $3 \mathrm{H}, J=7.0 \mathrm{~Hz}) ;{ }^{13} \mathrm{C} \mathrm{NMR}\left(100 \mathrm{MHz}, \mathrm{CDCl}_{3}\right) \delta 208,159.3,151.1,134.5,133.8,127.1,54.9,52.2$, 51.0, 41.7, 31.7, 30.2, 27.9, 24.6, 15.0, 14.4; IR (thin film) vmax 2935, 2875, 1710, 1454, 1375, 1281, 1257, 1165, $1032 \mathrm{~cm}^{-1}$; CIHRMS [M] calculated for $\mathrm{C}_{16} \mathrm{H}_{24} \mathrm{~N}_{2} \mathrm{O}: 261.1897$, found: 261.1946.

4-(6,7-Dimethyl-3,4-dihydro-2H-[1,5]naphthyridin-1-yl)-butan-2-one (20): To a high pressure tube o me were added 1,2,3,4-tetrahydro-6,7-dimethyl-1,5-naphthyridine ${ }^{4}$ (165.0 mg, $1.017 \mathrm{mmol}$ ),

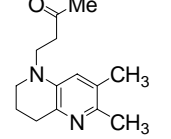
methyl vinyl ketone $(0.498 \mathrm{~mL}, 5.98 \mathrm{mmol})$, ytterbium(III) triflate $(63.1 \mathrm{mg}, 0.102$ $\mathrm{mmol})$, and methylene chloride $(1.5 \mathrm{~mL})$. The reaction mixture was heated at $40{ }^{\circ} \mathrm{C}$ for $12 \mathrm{~h}$ (monitored by ${ }^{1} \mathrm{H}$ NMR), then concentrated and chromatographed on silica gel (520 $10 \% \mathrm{MeOH}$ in $\left.\mathrm{CH}_{2} \mathrm{Cl}_{2}\right)$ to afford the desired product 20 as a yellow oil (160 $\mathrm{mg}, 0.692$ mmol, 68\%). ${ }^{1} \mathrm{H}$ NMR (400 MHz, $\left.\mathrm{CDCl}_{3}\right) \delta 6.56(\mathrm{~s}, 1 \mathrm{H}), 3.47(\mathrm{t}, 2 \mathrm{H}, J=6.8 \mathrm{~Hz}), 3.14(\mathrm{t}, 2 \mathrm{H}, J=5.6$ $\mathrm{Hz}), 2.80(\mathrm{t}, 2 \mathrm{H}, J=6.8 \mathrm{~Hz}), 2.65(\mathrm{t}, 2 \mathrm{H}, J=7.2 \mathrm{~Hz}), 2.27(\mathrm{~s}, 3 \mathrm{H}), 2.124(\mathrm{~s}, 3 \mathrm{H}), 2.116(\mathrm{~s}, 3 \mathrm{H}), 2.07$ (m, 2H), $1.92(\mathrm{~m}, 2 \mathrm{H}) ;{ }^{13} \mathrm{C}$ NMR $\left(100 \mathrm{MHz}, \mathrm{CDCl}_{3}\right) \delta 208.0,143.0,140.3,139.2,129.4,119.2,48.9$, 45.4, 39.7, 30.7, 30.4, 21.9, 21.0, 19.3; IR (thin film) vmax 3386, 2940, 1710, 1595, 1486, 1367, 1337, 1261, 1168, $867 \mathrm{~cm}^{-1}$; CIHRMS [M] $]^{+}$calculated for $\mathrm{C}_{14} \mathrm{H}_{20} \mathrm{~N}_{2} \mathrm{O}: 231.1584$, found: 231.1649 .

4-(2,3-Dimethyl-6,7,8,9-tetrahydro-pyrido[3,2-b]azepin-5-yl)-butan-2-one (21): To a high pressure

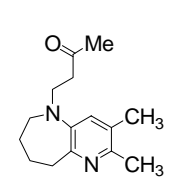

21 tube were added 2,3-dimethyl-6,7,8,9-tetrahydro-5H-pyrido[3,2-b]azepine $(180.0 \mathrm{mg}$, $1.021 \mathrm{mmol})$, methyl vinyl ketone $(0.5 \mathrm{~mL}, 6.01 \mathrm{mmol})$, ytterbium(III) triflate $(63.3 \mathrm{mg}$, $0.102 \mathrm{mmol})$, and methylene chloride $(1.5 \mathrm{~mL})$. The reaction mixture was heated at 40 ${ }^{\circ} \mathrm{C}$ for $12 \mathrm{~h}$ (monitored by ${ }^{1} \mathrm{H} \mathrm{NMR}$ ) then concentrated and chromatographed on silica gel $\left(5-10 \% \mathrm{MeOH}\right.$ in $\left.\mathrm{CH}_{2} \mathrm{Cl}_{2}\right)$ to afford the desired product 21 as a yellow oil $(178 \mathrm{mg}$, $0.725 \mathrm{mmol}, 71 \%) .{ }^{1} \mathrm{H}$ NMR $\left(400 \mathrm{MHz}, \mathrm{CDCl}_{3}\right) \delta 6.93(\mathrm{~s}, 1 \mathrm{H}), 3.40(\mathrm{t}, 2 \mathrm{H}, J=6.8$ $\mathrm{Hz}), 2.88(\mathrm{~m}, 2 \mathrm{H}), 2.66(\mathrm{t}, 2 \mathrm{H}, J=7.2 \mathrm{~Hz}), 2.37(\mathrm{~s}, 3 \mathrm{H}), 2.20(\mathrm{~s}, 3 \mathrm{H}), 2.12(\mathrm{~s}, 3 \mathrm{H}), 1.68(\mathrm{~m}, 2 \mathrm{H}), 1.61$ $(\mathrm{m}, 2 \mathrm{H}) ;{ }^{13} \mathrm{C} \mathrm{NMR}\left(100 \mathrm{MHz}, \mathrm{CDCl}_{3}\right) \delta 208.1,153.2,148.1,145.0,129.0,126.8,54.8,48.6,43.0$, $36.9,30.35,30.26,24.3,21.5,19.1$; IR (thin film) vmax 3396, 2925, 1711, 1590, 1469, 1449, 1361, 1241, 1165, 1129, 992, $886 \mathrm{~cm}^{-1}$; CIHRMS [M] ${ }^{+}$calculated for $\mathrm{C}_{15} \mathrm{H}_{22} \mathrm{~N}_{2} \mathrm{O}$ : 246.1732, found: 246.1715 .

\footnotetext{
${ }^{3}$ Matsubara, S.; Yoshioka, M.; Utimoto, K. Chem. Lett. 1994, 5, 827-830.

${ }^{4}$ a) Neipp, C. E.; Ranslow, P. B.; Wan, Z.; Snyder, J. K. Tetrahedron Lett. 1997, 43, 7499-7502; b) Lahue, B. R.; Lo, S.M.; Wan, Z.-K.; Woo, G. H. C.; Snyder, J. K. J. Org. Chem. 2004, 69, 7171-7182.
} 
Scheme 3. Synthesis of keto-pyran 22.
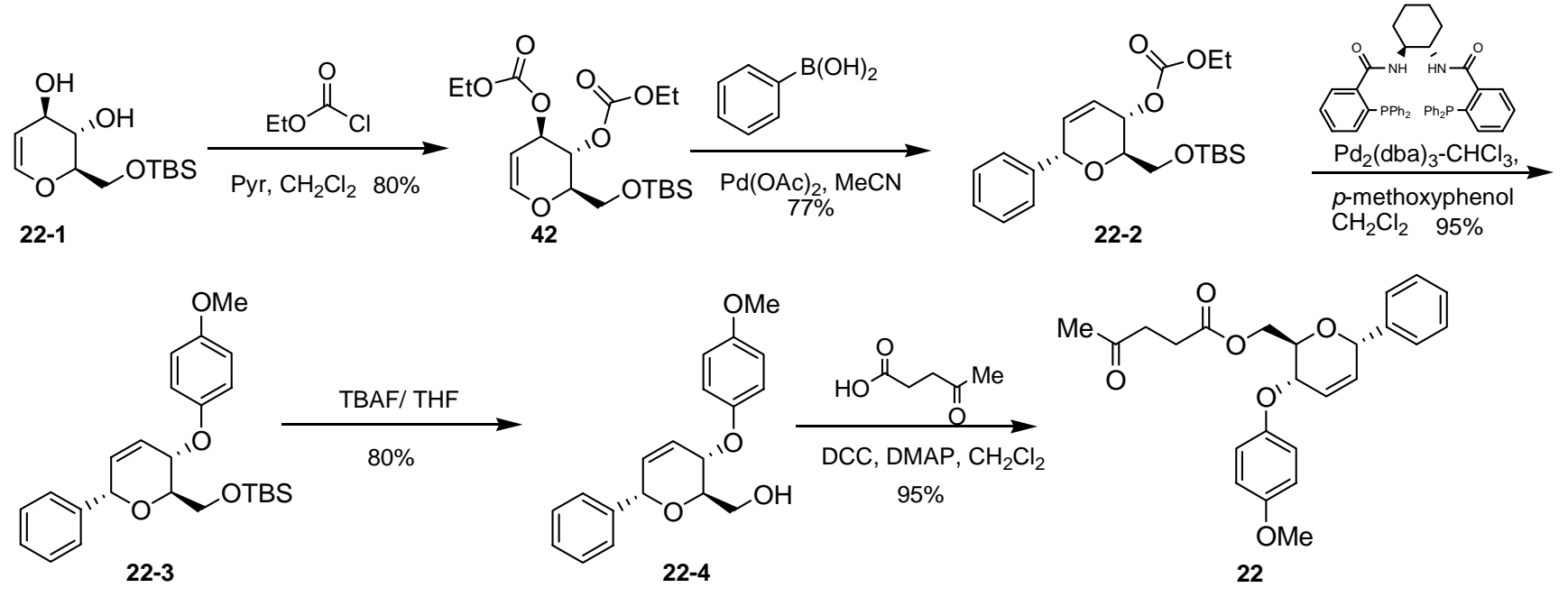

Figure 1. X-ray structure for derivative 22-5.

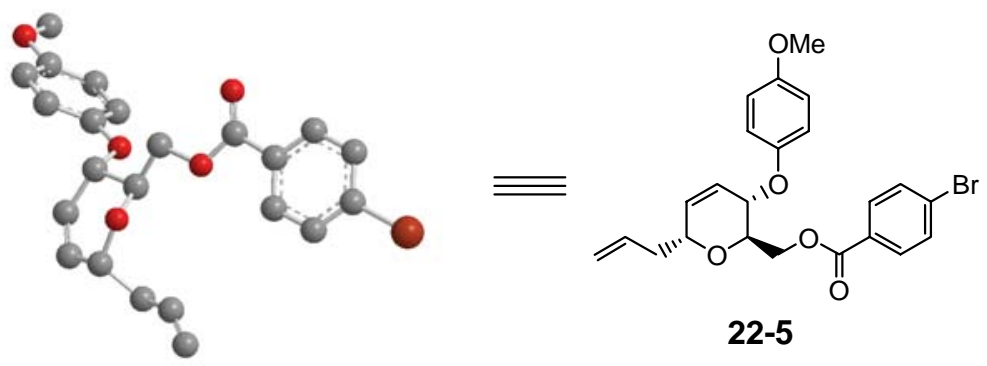

((2R,3S,6S)-3-(4-Methoxyphenoxy)-3,6-dihydro-6-phenyl-2H-pyran-2-yl)methyl 4-oxopentanoate

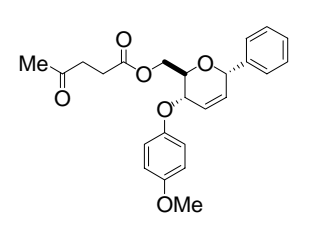

22

(22): 2-(Tert-butyl-dimethyl-silanyloxymethyl)-3,4-dihydro-2 $\mathrm{H}$-pyran-3,4-diol (22-1) $(1.00 \mathrm{~g}, 3.85 \mathrm{mmol})$ was dissolved in pyridine $(1.55 \mathrm{~mL}, 19.2 \mathrm{mmol})$ and methylene chloride $(5 \mathrm{~mL})$ under an argon atmosphere. The reaction was cooled to $0{ }^{\circ} \mathrm{C}$ and ethyl chloroformate $(1.47 \mathrm{~mL}, 15.4 \mathrm{mmol})$ was added. The reaction was allowed to stir overnight, diluted with ethyl acetate, washed with sodium bicarbonate (3x) and brine (1x), dried with $\mathrm{MgSO}_{4}$, and concentrated in vacuo. Chromatography over silica gel with $0-15 \%$ ethyl acetate / hexanes provided compound 42 . The general procedure of Ramnauth et. al. ${ }^{5}$ was utilized for arylation of 42. Carbonic acid 2-(tert-butyldimethyl-silanyloxymethyl)-4-ethoxycarbonyloxy-3,4-dihydro-2 $H$-pyran- 3-yl ester ethyl ester (42) $(1.00 \mathrm{~g}, 2.47 \mathrm{mmol})$ was dissolved in acetonitrile $(10 \mathrm{~mL})$ open to the atmosphere. To the reaction was added phenylboronic acid $(1700 \mathrm{mg}, 14.0 \mathrm{mmol})$ and palladium acetate $(140 \mathrm{mg}, 0.62 \mathrm{mmol})$. The reaction was stirred overnight, diluted with methylene chloride, washed with sodium bicarbonate and brine, concentrated onto silica, and chromatographed with 0-10\% ethyl acetate/hexanes to afford 22-2. Carbonic acid 2-(tert-butyl-dimethyl-silanyloxymethyl)-6-phenyl-3,6-dihydro-2H-pyran-3-yl ester ethyl ester (22-2) $(815 \mathrm{mg}, 2.08 \mathrm{mmol})$, tris(dibenzylideneacetone)dipalladium (0) chloroform adduct (54 mg, $0.052 \mathrm{mmol})$, and $(1 S, \quad 2 S)-(-)-1,2$-diaminocyclohexane- $N, N^{\prime}$-bis $\quad\left(2^{\prime}-\right.$ diphenylphosphinobenzoyl) (70 mg, $0.1 \mathrm{mmol})$ were dissolved in degassed methylene chloride $(10 \mathrm{~mL})$ under an atmosphere of argon. After stirring for 30 minutes, $p$-methoxyphenol (283 $\mathrm{mg}, 2.28 \mathrm{mmol})$ was added to the reaction mixture. The reaction was heated in the CEM Discover Explorer microwave system for 10 minutes at $100{ }^{\circ} \mathrm{C}$ using PowerMax mode $(300 \mathrm{~W})$. The reaction was concentrated onto silica gel and chromatographed with $0-10 \%$ ethyl acetate/hexanes to yield 22-3 as a colorless oil. ${ }^{1} \mathrm{H}$

\footnotetext{
${ }^{5}$ Ramnauth, J.; Poulin, O.; Rakhit, S.; Maddaford, S. P. Org. Lett. 2001, 3, 2013-2015.
} 
NMR $\left(400 \mathrm{MHz}, \mathrm{CDCl}_{3}\right) \delta 0.01(\mathrm{~s}, 1 \mathrm{H}), 0.02(\mathrm{~s}, 1 \mathrm{H}), 0.87(\mathrm{~s}, 9 \mathrm{H}), 3.78(\mathrm{~m}, 4 \mathrm{H}), 3.85(\mathrm{t}, 2 \mathrm{H}, J=6.0$ $\mathrm{Hz}), 4.78(\mathrm{~d}, 1 \mathrm{H}, J=7.2 \mathrm{~Hz}), 6.12(\mathrm{~m}, 2 \mathrm{H}), 6.87(\mathrm{dd}, 2 \mathrm{H}, J=9.2,29.2 \mathrm{~Hz}), 7.32(\mathrm{~m}, 3 \mathrm{H}), 7.48(\mathrm{~d}, 2 \mathrm{H}$, $J=7.2 \mathrm{~Hz}) ;{ }^{13} \mathrm{C} \mathrm{NMR}\left(100 \mathrm{MHz}, \mathrm{CDCl}_{3}\right) \delta-5.4,18.3,25.9,55.6,62.9,72.2,73.8,114.6,116.8,125.8$, $127.8,128.3,130.6,139.5,151.5,154.0$; IR (thin film) vmax 3029, 2955, 2924, 2850, 1507, 1460, 1227, 1087, 1036, 835, 781; CIHRMS [M] ${ }^{+}$calculated for $\mathrm{C}_{25} \mathrm{H}_{34} \mathrm{O}_{4} \mathrm{Si}: 426.2226$, found: 426.2235; $[\alpha]_{\mathrm{D}}^{23}=49.2^{\circ}\left(\mathrm{c}=0.255, \mathrm{CH}_{2} \mathrm{Cl}_{2}\right)$. tert-Butyl-[3-(4-methoxy-phenoxy)-6-phenyl-3,6-dihydro- $2 \mathrm{H}$ pyran-2-ylmethoxy]-dimethyl-silane (22-3) $(328 \mathrm{mg}, 0.768 \mathrm{mmol})$ was dissolved in tetrahydrofuran (4 $\mathrm{mL}$ ) under an atmosphere of argon. A solution of $1 \mathrm{M}$ of tetra- $n$-butylammonium fluoride in tetrahydrofuran $(0.846 \mathrm{~mL})$ was added. After 5 minutes the reaction was concentrated onto silica gel and chromatographed with 6-40\% ethyl acetate/hexanes to afford 22-4. The stereochemistry of 22-4 was established based on analogy to the related structure 22-5 (Fig. 1). [3-(4-Methoxy-phenoxy)-6phenyl-3,6-dihydro-2H-pyran-2-yl]-methanol (22-4) (180.0 mg, $0.576 \mathrm{mmol})$, 4-oxopentanoic acid (73.6 mg, $0.634 \mathrm{mmol})$, 4-dimethylaminopyridine $(3.5 \mathrm{mg}, 0.029 \mathrm{mmol})$, and methylene chloride $(2.00 \mathrm{~mL})$ were added to the reaction flask. The solution was cooled to $0{ }^{\circ} \mathrm{C}$ and $N, N^{\prime}-$ dicyclohexylcarbodiimide $(125 \mathrm{mg}, 0.605 \mathrm{mmol})$ was added. The reaction mixture was stirred for $3 \mathrm{~h}$ $\left(0{ }^{\circ} \mathrm{C}\right.$ to $\left.\mathrm{rt}\right)$, filtered, and subjected to flash chromatography on silica gel $(0-50 \%$ EtOAc/hexane $)$ to afford the desired product 22 as a colorless oil $(220 \mathrm{mg}, 0.547 \mathrm{mmol}, 95 \%) .{ }^{1} \mathrm{H} \mathrm{NMR}(400 \mathrm{MHz}$, $\left.\mathrm{CDCl}_{3}\right) \delta 7.31(\mathrm{~m}, 5 \mathrm{H}), 6.83(\mathrm{~m}, 4 \mathrm{H}), 7.47(\mathrm{~d}, 2 \mathrm{H}, J=7.2 \mathrm{~Hz}), 6.16(\mathrm{~m}, 2 \mathrm{H}), 5.34(\mathrm{bs}, 1 \mathrm{H}), 4.26(\mathrm{~m}$, 2H), $3.86(\mathrm{~m}, 1 \mathrm{H}), 3.76(\mathrm{~s}, 3 \mathrm{H}), 2.68(\mathrm{t}, 2 \mathrm{H}, J=6.0 \mathrm{~Hz}), 2.58(\mathrm{t}, 2 \mathrm{H}, J=6.4 \mathrm{~Hz}), 2.13(\mathrm{~s}, 3 \mathrm{H}) ;{ }^{13} \mathrm{C}$ NMR $\left(100 \mathrm{MHz}, \mathrm{CDCl}_{3}\right) \delta 206.6,172.6,154.5,151.2,139.0,130.4,128.6,128.2,128.1,160.0,117.4$, $114.9,74.3,69.8,69.4,63.8,55.7,37.9,30.0,27.9$; IR (thin film) vmax 3034, 2999, 2953, 2909, 2835 , $1738,1505,1452,1409,1359,1307,1225,1160,1100,1064,828,740 \mathrm{~cm}^{-1}$; CIHRMS [M+H] calculated for $\mathrm{C}_{24} \mathrm{H}_{27} \mathrm{O}_{6}: 411.1808$, found: $411.1824 ;[\alpha]_{\mathrm{D}}{ }^{23}=59.2^{\circ}\left(\mathrm{c}=0.382, \mathrm{CH}_{2} \mathrm{Cl}_{2}\right)$.

Scheme 4. Synthesis of 23.

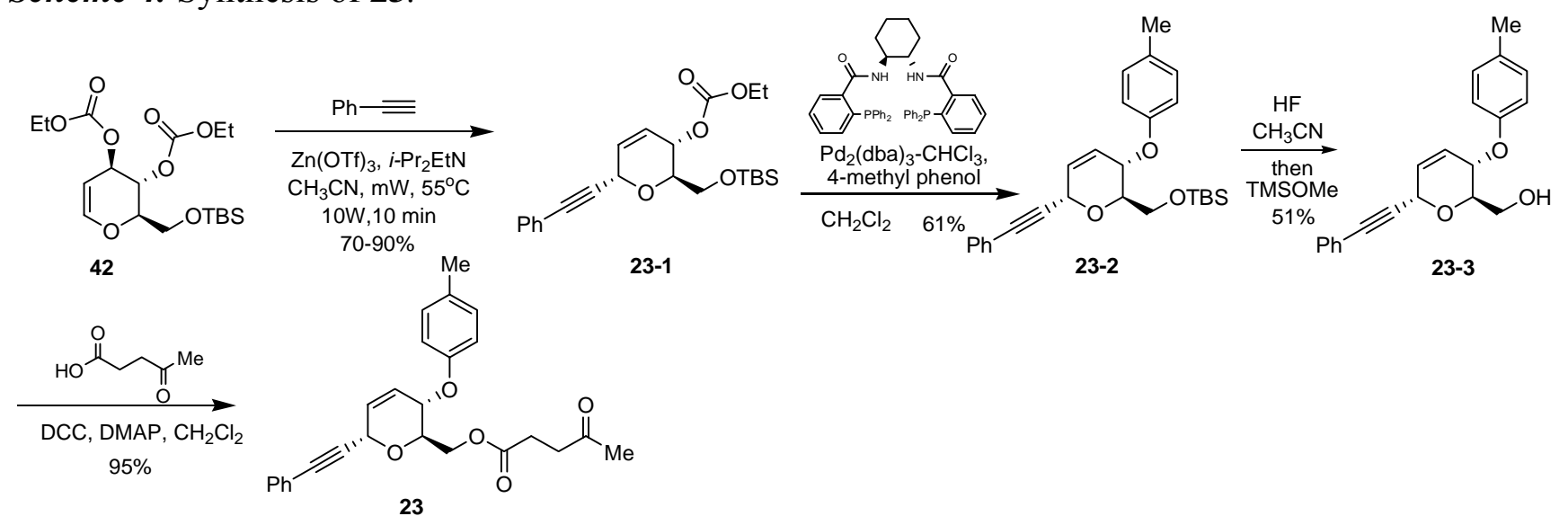

(2R,3S,6S)-3-(p-Tolyloxy)-3,6-dihydro-6-(2-phenylethynyl)-2H-pyran-2-yl)methyl 4-

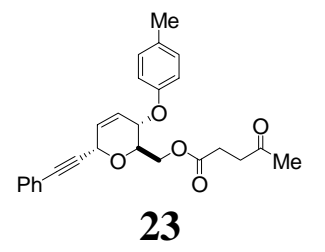

oxopentanoate (23): Phenylacetylene $(200 \mu \mathrm{L}, 1.85 \mathrm{mmol})$ was dissolved in acetonitrile $(5 \mathrm{~mL})$ under an atmosphere of argon. To the reaction was added $N, N$-diisopropylethylamine $(650 \mu \mathrm{L}, 3.7 \mathrm{mmol})$ and zinc triflate $(650 \mathrm{mg}, 3.7$ $\mathrm{mmol})$. The reaction was stirred for 15 minutes at $\mathrm{rt}$ and carbonic acid 2-(tertbutyl-dimethyl-silanyloxymethyl)-4-ethoxycarbonyloxy-3,4-dihydro- $2 \mathrm{H}$ pyran- 3-yl ester ethyl ester (42) (500 mg, $1.24 \mathrm{mmol}$ ) was added. The reaction was heated in a microwave vessel for 15 minutes at $55^{\circ} \mathrm{C}(10 \mathrm{~W})$. The reaction was concentrated onto silica gel and chromatographed on silica gel eluting with $0-20 \%$ ethyl acetate/hexanes to afford 23-1. Carbonic acid 2-(tert-butyl-dimethyl-silanyloxymethyl)-6- 
phenylethynyl-3,6-dihydro-2H-pyran-3-yl ester ethyl ester (23-1) (314 mg, $0.724 \mathrm{mmol}$ ) was dissolved in degassed methylene chloride $(3 \mathrm{~mL})$ under an atmosphere of argon followed by the addition of $(1 S, 2 S)$-(-)-1,2-diaminocyclohexane- $N, N^{\prime}$-bis (2'-diphenylphosphinobenzoyl) (30 $\left.\mathrm{mg}, 0.04 \mathrm{~mol}\right)$ and tris(dibenzylideneacetone)dipalladium(0) chloroform adduct $(20 \mathrm{mg}, 0.019 \mathrm{mmol})$. The deep red reaction was stirred under argon for 20 minutes and turned yellow. To the reaction vessel was added 4methylphenol and the reaction was heated in the Discovery Explorer microwave system $(80 \mathrm{~mL}$ vessel) for 10 minutes at $100{ }^{\circ} \mathrm{C}(300 \mathrm{~W})$. The resulting mixture was concentrated onto silica gel and was chromatographed eluting with 0-20\% ethyl acetate/hexanes to provide 23-2. Tert-butyl-dimethyl-(6phenylethynyl-3-p-tolyloxy-3,6-dihydro-2H-pyran-2-ylmethoxy)-silane (23-2) (482 mg, $1.11 \mathrm{mmol})$ was dissolved in acetonitrile $(10 \mathrm{~mL})$ and the solution was cooled to $0^{\circ} \mathrm{C}$. To the reaction was added a solution of $48 \%$ aq. hydrofluoric acid $(127 \mu \mathrm{L}, 3.66 \mathrm{mmol})$. The reaction was stirred at rt for 100 minutes, methoxytrimethylsilane $(1.0 \mathrm{~mL}, 10 \mathrm{~mol})$ was added, and the reaction was stirred overnight. The reaction was then concentrated onto silica gel and chromatographed with 5-40 \% ethyl acetate/hexanes to provide 23-3. The stereochemical assignment for 23-3 was based on analogy to the structures of 22-4 and 22-5. (6-Phenylethynyl-3-p-tolyloxy-3,6-dihydro-2H-pyran-2-yl)-methanol (233) $(175.0 \mathrm{mg}, 0.546 \mathrm{mmol})$, 4-oxopentanoic acid (69.8 $\mathrm{mg}, 0.601 \mathrm{mmol})$, 4-dimethylaminopyridine (3.3 $\mathrm{mg}, 0.027 \mathrm{mmol})$, and methylene chloride $(2.0 \mathrm{~mL})$ were added to a reaction flask. The solution was cooled to $0{ }^{\circ} \mathrm{C}$ and $N, N^{\prime}$-dicyclohexylcarbodiimide $(118 \mathrm{mg}, 0.574 \mathrm{mmol})$ was added. The reaction mixture was stirred for $3 \mathrm{~h}\left(0{ }^{\circ} \mathrm{C}\right.$ to $\left.\mathrm{rt}\right)$, filtered, and subjected to flash chromatography on silica gel (0-40\% EtOAc/hexane) to afford 23 as a colorless oil $(215 \mathrm{mg}, 0.52 \mathrm{mmol}, 95 \%) .{ }^{1} \mathrm{H}$ NMR $\left(400 \mathrm{MHz}, \mathrm{CDCl}_{3}\right) \delta 7.45(\mathrm{~m}, 2 \mathrm{H}), 7.31(\mathrm{~m}, 3 \mathrm{H}), 7.08(\mathrm{~d}, 2 \mathrm{H}, J=7.6 \mathrm{~Hz}), 6.81(\mathrm{~d}, 2 \mathrm{H}, J=8.8 \mathrm{~Hz})$, $6.01(\mathrm{dd}, 1 \mathrm{H}, J=2.0,11.6 \mathrm{~Hz}), 5.96(\mathrm{ddd}, 1 \mathrm{H}, J=1.6,3.2,10.4 \mathrm{~Hz}), 5.20(\mathrm{~m}, 1 \mathrm{H}), 4.76(\mathrm{~m}, 1 \mathrm{H}), 4.35$ $(\mathrm{m}, 1 \mathrm{H}), 4.28(\mathrm{~m}, 12 \mathrm{H}), 2.70(\mathrm{t}, 2 \mathrm{H}, J=6.8 \mathrm{~Hz}) ; 2.62(\mathrm{t}, 2 \mathrm{H}, J=6.0 \mathrm{~Hz}), 2.27(\mathrm{~s}, 3 \mathrm{H}), 2.11(\mathrm{~s}, 3 \mathrm{H})$; ${ }^{13} \mathrm{C} \mathrm{NMR}\left(100 \mathrm{MHz}, \mathrm{CDCl}_{3}\right) \delta 206.6,172.6,154.9,132.0,131.1,130.3,128.8,128.7,128.4,125.8$, $122.3,115.8,86.6,85.1,70.9,68.7,64.9,63.8,38.0,29.9,28.0,20.6$; IR (thin film) vmax 3032, 2953, $2922,1739,1720,1613,1509,1358,1232,1174,1158,1083,1031,817,759 \mathrm{~cm}^{-1}$; CIHRMS [M+H] calculated for $\mathrm{C}_{26} \mathrm{H}_{27} \mathrm{O}_{5}$ : 419.1858, found: $419.1818 ;[\alpha]_{\mathrm{D}}^{23}=-51.0^{\circ}\left(\mathrm{c}=0.382, \mathrm{CH}_{2} \mathrm{Cl}_{2}\right)$.

Compound was prepared according to: De Raadt, A.; Ferrier, R. J. Carbohydr. Res. 1991, 216, 93-107. $\mathrm{AcO}^{\mathrm{AcO}} \mathrm{C} \mathrm{CHO}$

24

$(E, 5 R, 6 S)-M e t h y l \quad 6-f o r m y l-5-m e t h y l-6-(t o s y l a m i n o) h e x-3-e n o a t e \quad(25): \quad(E, 5 R, 6 S)$-methyl $\quad 7$ hydroxy-5-methyl-6-(tosylamino)hept-3-enoate ${ }^{6}(200 \mathrm{mg}, 0.59 \mathrm{mmol})$ was $\mathrm{OHC}_{\text {Me }}^{\mathrm{NHTS}} \overbrace{\text { coome }}$ dissolved in $\mathrm{CH}_{2} \mathrm{Cl}_{2}(3 \mathrm{~mL})$ and the solution was cooled to $0^{\circ} \mathrm{C}$. To the solution was added $4 \AA$ molecular sieves $(200 \mathrm{mg})$, 4-methylmorpholine $N$-oxide (76 mg, 25 $0.649 \mathrm{mmol})$, and tetrapropylammonium perruthenate $(20 \mathrm{mg}, 0.059 \mathrm{mmol}){ }^{7} \mathrm{The}$ reaction was stirred for $3 \mathrm{~h}\left(0{ }^{\circ} \mathrm{C}-\mathrm{rt}\right)$ and the solution was eluted through a silica

plug (5 g) using EtOAc as solvent. The eluant was concentrated and the resulting aldehyde (25) was used without further purification.

\footnotetext{
${ }^{6}$ For preparation, please see: Su, Q.; Beeler, A. B.; Lobkovsky, E.; Porco, J. A., Jr.; Panek, J. S. Org. Lett. 2003, 5, 21492152.

${ }^{7}$ Ley, S. V.; Norman, J.; Griffith, W. P.; Marsden, S. P. Synthesis 1994, 7, 639-666.
} 


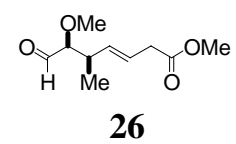

(R,E)-Methyl 5-((S)-formyl(methoxy)methyl)hex-3-enoate $\quad(26): \quad(E, 5 R, 6 S)$ methyl 7-hydroxy-6-methoxy-5-methylhept-3-enoate ${ }^{4}(202 \mathrm{mg}, 1.0 \mathrm{mmol})$ was dissolved in $\mathrm{CH}_{2} \mathrm{Cl}_{2}(3 \mathrm{~mL})$ and the solution was cooled to $0{ }^{\circ} \mathrm{C}$. To the solution was added $4 \AA$ molecular sieves $(200 \mathrm{mg})$, 4-methylmorpholine $N$-oxide (123 mg, 1.05 $\mathrm{mmol})$, and tetrapropylammonium perruthenate $(35.1 \mathrm{mg}, 0.1 \mathrm{mmol})$. The reaction was stirred for $3 \mathrm{~h}\left(0^{\circ} \mathrm{C}-\mathrm{rt}\right)$ and the solution was eluted through a silica plug $(5 \mathrm{~g})$ using EtOAc. The eluant was concentrated and the resulting aldehyde (26) was used in oxime formation without further purification.

Scheme 5. Synthesis of aldehyde 27.

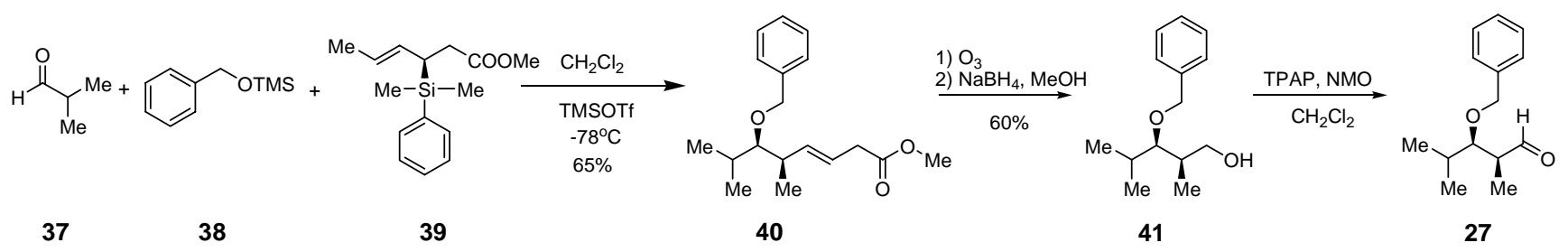

$(E, 5 S, 6 S)$-Methyl 6-(benzyloxy)-5,7-dimethyloct-3-enoate (27): A solution of isobutyraldehyde (37) $(0.64 \mathrm{~mL}, 7.1 \mathrm{mmol})$, benzyloxytrimethylsilane (38), and chiral silane $39(2.8 \mathrm{~mL}, 14.0$ mol) in dry methylene chloride $(10 \mathrm{~mL})$ was cooled to $-78{ }^{\circ} \mathrm{C}$. To the reaction was added trimethylsilyl trifluoromethanesulfonate $(1.4 \mathrm{~mL}, 7.6 \mathrm{mmol})$ dropwise. ${ }^{8}$ The reaction was stirred at $-78{ }^{\circ} \mathrm{C}$ for $18 \mathrm{~h}$ before being diluted with sat. $\mathrm{NaHCO}_{3}$. The mixture was stirred for 2 minutes and extracted with $\mathrm{Et}_{2} \mathrm{O}$. The organic layers were dried with magnesium sulfate, filtered, and concentrated. Silica gel chromatography with $15 \%$ EtOAc/hexanes afforded $\mathbf{4 0}$ as a colorless oil. To a 1-neck round-bottom flask was added $(E, 5 S, 6 S)$-methyl 6-(benzyloxy)-5,7-dimethyloct-3-enoate (40) $(700 \mathrm{mg}, 2.4 \mathrm{mmol})$ and methanol (10 $\mathrm{mL}$ ). The reaction was cooled to $-78{ }^{\circ} \mathrm{C}$ and ozone was bubbled into the solution. After 5 minutes the reaction was quenched with sodium tetrahydroborate ( $100 \mathrm{mg}, 2.6 \mathrm{mmol})$, warmed to $\mathrm{rt}$, and stirred for $4 \mathrm{~h}$. The solvent was removed and the crude residue was purified by silica gel chromatography $(0-50 \%$ EtOAc/Hexane) to afford 41 as a colorless oil (320 mg, $1.44 \mathrm{mmol}, 60 \%) .{ }^{1} \mathrm{H}$ NMR (400 MHz, $\mathrm{CDCl}_{3}$ ) $\delta 7.35(\mathrm{~m}, 5 \mathrm{H}), 4.60(\mathrm{q}, 2 \mathrm{H}, J=11.2 \mathrm{~Hz}), 3.56(\mathrm{ddd}, 2 \mathrm{H}, J=7.6,2.8,6.0,5.6,4.8 \mathrm{~Hz}), 3.24(\mathrm{dd}, 1 \mathrm{H}, J$ $=3.2,4.8 \mathrm{~Hz}), 2.31(\mathrm{bs}, 1 \mathrm{H}), 1.98(\mathrm{~m}, 2 \mathrm{H}), 1.04(\mathrm{~d}, 3 \mathrm{H}, J=6.8 \mathrm{~Hz}), 0.93(\mathrm{~d}, 3 \mathrm{H}, J=3.6 \mathrm{~Hz}), 0.91(\mathrm{~d}$, $3 \mathrm{H}, J=2.8 \mathrm{~Hz}) ;{ }^{13} \mathrm{CNMR}\left(100 \mathrm{MHz}, \mathrm{CDCl}_{3}\right) \delta 139.0,128.5,127.8,127.7,85.8,74.8,66.3,37.7,31.0$, 19.9, 19.6, 11.0; IR (thin film) vmax 3396, 3031, 2963, 2931, 1496, 1095, 1067, $1029 \mathrm{~cm}^{-1}$. CIHRMS $[\mathrm{M}]^{+}$calculated for $\mathrm{C}_{15} \mathrm{H}_{23} \mathrm{O}_{3}$ : 223.1628, found: 223.1631; $[\alpha]_{\mathrm{D}}{ }^{23}=-8.6^{\circ}\left(\mathrm{c}=0.664, \mathrm{CH}_{2} \mathrm{Cl}_{2}\right)$. Compound $41(220 \mathrm{mg}, 1.0 \mathrm{mmol})$ was dissolved in $\mathrm{CH}_{2} \mathrm{Cl}_{2}(3 \mathrm{~mL})$ and the solution was cooled to $0^{\circ} \mathrm{C}$. To the solution was added $4 \AA$ molecular sieves $(220 \mathrm{mg}$ ), 4-methylmorpholine $N$-oxide (123 mg, 1.05 $\mathrm{mmol}$ ) and tetrapropylammonium perruthenate $(35 \mathrm{mg}, 0.1 \mathrm{mmol})$. The reaction was stirred for $3 \mathrm{~h}$ $\left(0^{\circ} \mathrm{C}-\mathrm{rt}\right)$ and the solution was eluted through a silica plug $(5 \mathrm{~g})$ using EtOAc. The eluant was concentrated and the resulting aldehyde (27) was used without further purification.

${ }^{8}$ Panek, J. S.; Yang, M.; Xu, F. J. Org. Chem. 1992 , 57, 5790-5792. 
Scheme 6. Synthesis of 28.

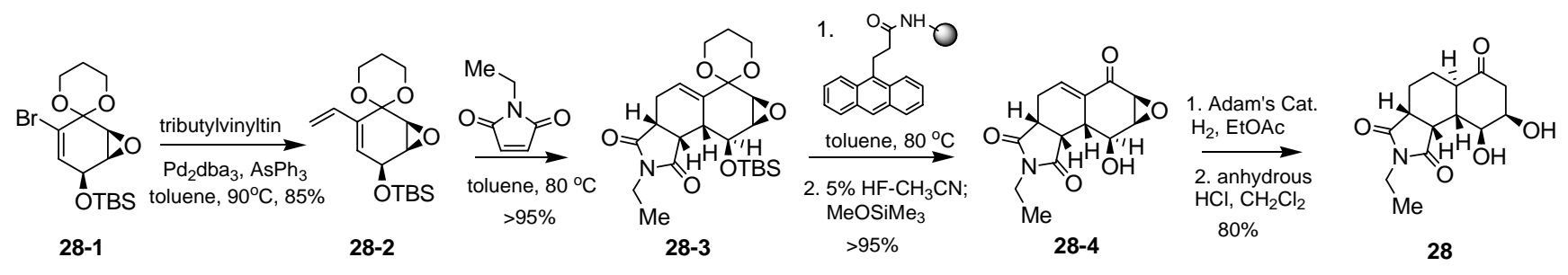

Figure 2. X-ray structure of 28-4.

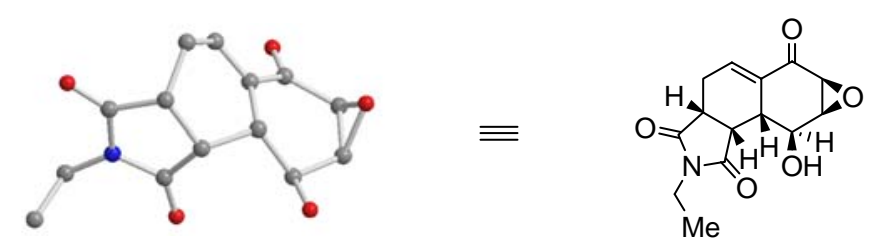

(3aR,5aR,8R,9S,9aS,9bS)-2-Ethyl-octahydro-8,9-dihydroxy-2H-benzo[e]isoindole-1,3,6(9bH)-

trione (28): $\mathrm{Pd}_{2}(\mathrm{dba})_{3}(956 \mathrm{mg}, 1.04 \mathrm{mmol})$, and $\mathrm{AsPh}_{3}(1.652 \mathrm{~g}, 5.40 \mathrm{mmol})$ were

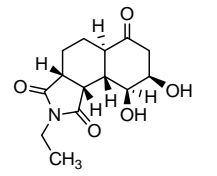

28 placed in a $250 \mathrm{~mL}$ flame-dried round bottom flask. To this mixture was added $20 \mathrm{~mL}$ of anhydrous toluene and the reaction was stirred at $\mathrm{rt}$ for $30 \mathrm{~min}$. To the reaction mixture was added bromide 28-1 $(6.668 \mathrm{~g}, 17.7 \mathrm{mmol})$ in $30 \mathrm{~mL}$ of anhydrous toluene, followed by tributylvinyltin $(6.2 \mathrm{~mL}, 21.2 \mathrm{mmol})$. The reaction mixture was stirred at $90{ }^{\circ} \mathrm{C}$ for $24 \mathrm{~h}$ and concentrated in vacuo. Purification on silica gel $(10 \%$ EtOAc in hexane) provided $4.884 \mathrm{~g}(15.0 \mathrm{mmol}, 85 \%)$ of diene $\mathbf{2 8 - 2}$ as a yellow solid. m.p. 65 $66{ }^{\circ} \mathrm{C} .{ }^{1} \mathrm{H}$ NMR $\left(400 \mathrm{MHz}, \mathrm{CDCl}_{3}\right): \delta 6.44(1 \mathrm{H}, \mathrm{dd}, J=11.2,17.6), 5.61(1 \mathrm{H}, \mathrm{s}), 5.53(1 \mathrm{H}, \mathrm{d}, J=$ 17.2), $5.12(1 \mathrm{H}, \mathrm{d}, J=11.2), 4.67(1 \mathrm{H}, \mathrm{s}), 4.32(3 \mathrm{H}, \mathrm{m}), 4.00(4 \mathrm{H}, \mathrm{m}), 3.45(1 \mathrm{H}, \mathrm{m}), 2.19(1 \mathrm{H}, \mathrm{m}), 1.52$ $(1 \mathrm{H}, \mathrm{d}, J=12.0), 0.91(9 \mathrm{H}, \mathrm{s}), 0.13(6 \mathrm{H}, \mathrm{s}) \mathrm{ppm} ;{ }^{13} \mathrm{C} \mathrm{NMR}\left(75 \mathrm{MHz}, \mathrm{CDCl}_{3}\right): \delta 135.6,132.7,126.0$, 122.7, 115.9, 93.8, 92.5, 67.0, 66.1, 61.4, 60.9, 54.1, 49.3, 25.7 24.3, 18.0, -4.6, -4.8 ppm; IR (neat): $2955,2857,1471,1384,1136,1005 \mathrm{~cm}^{-1}$; HRMS (CI) [M+H] ${ }^{+}$: calcd. for $\mathrm{C}_{17} \mathrm{H}_{28} \mathrm{O}_{4} \mathrm{Si} 325.1836$, found 325.1809. $[\alpha]_{\mathrm{D}}{ }^{23}=+52^{\circ}\left(\mathrm{c}=1.0 \mathrm{CHCl}_{3}\right)$. Diene 28-2 $(324 \mathrm{mg}, 1.0 \mathrm{mmol})$ and $N$-ethyl maleimide (187mg, $1.5 \mathrm{mmol})$ were placed in a Quest $2105 \mathrm{~mL}$ reaction vessel, and dissolved in anhydrous toluene $(5 \mathrm{~mL})$. The reaction mixture was agitated at $80^{\circ} \mathrm{C}$ for $12 \mathrm{~h}$ before 2.0 equiv. of PS-anthracene dienophile scavenger resin $(1 \mathrm{~g}, 1 \mathrm{mmol}, 1.08 \mathrm{mmol} / \mathrm{g})^{9}$ was added to the solution. The reaction mixture was agitated at $80{ }^{\circ} \mathrm{C}$ for an additional $6 \mathrm{~h}$, filtered, and concentrated in vacuo. The residue was dissolved in HPLC grade acetonitrile $(10 \mathrm{~mL})$ and to this solution was added $48 \%$ aq. HF $(1 \mathrm{~mL})$. The reaction mixture was stirred at $\mathrm{rt}$ for $3 \mathrm{~h}$, then quenched by addition of methoxytrimethylsilane $\mathrm{e}^{10}$ $(0.23 \mathrm{~mL}, 1.67 \mathrm{mmol})$. The mixture was stirred at $\mathrm{rt}$ for another $3 \mathrm{~h}$, then concentrated in vacuo to afford 28-4. Single x-ray crystal structure analysis confirmed the stereochemistry of 28-4 (Fig. 2). m.p. 150.0-151.0 ${ }^{\circ} \mathrm{C} ;{ }^{1} \mathrm{H}$ NMR $\left(400 \mathrm{MHz}, \mathrm{CDCl}_{3}\right): \delta 6.91(1 \mathrm{H}, \mathrm{td}, J=3.2,6.8), 4.83(1 \mathrm{H}, \mathrm{dd}, J=5.6,10.8)$, $3.81(1 \mathrm{H}, \mathrm{d}, J=4.4), 3.46(4 \mathrm{H}, \mathrm{m}), 3.17(2 \mathrm{H}, \mathrm{m}), 2.98(1 \mathrm{H}, \mathrm{dd}, J=6.0,17.2), 2.88(1 \mathrm{H}, \mathrm{m}), 2.26(1 \mathrm{H}$, m), $1.07(3 \mathrm{H}, \mathrm{t}, J=7.2) \mathrm{ppm} ;{ }^{13} \mathrm{C} \mathrm{NMR}\left(75 \mathrm{MHz}, \mathrm{CDCl}_{3}\right): \delta 188.3,174.0,173.6,133.1,130.7,60.9$, 54.9, 50.7, 34.3, 32.9, 32.5, 29.5, 19.9, 8.3 ppm; IR (neat): 3446, 2980, 2941, 1699, 1405, 1340, 1228, $1170 \mathrm{~cm}^{-1}$; HRMS (CI) $[\mathrm{M}+\mathrm{H}]^{+}$: calcd. for $\mathrm{C}_{14} \mathrm{H}_{16} \mathrm{NO}_{5} 278.1029$, found $278.1030 .[\alpha]_{\mathrm{D}}{ }^{23}=-46^{\circ}(\mathrm{c}=$ $\left.1.0 \mathrm{CHCl}_{3}\right)$. 28-4 (248 mg, $\left.0.89 \mathrm{mmol}\right)$ and Adam's catalyst $(13.0 \mathrm{mg}, 5$ w.t. \%) were dissolved in distilled EtOAc $(10 \mathrm{~mL})$, and the reaction mixture was stirred under a hydrogen atmosphere at room

${ }^{9}$ For preparation and characterization of the scavenger resin, see: Lei, X.; Porco, J. A., Jr. Org. Lett. 2004, 6, 795-798.
${ }^{10}$ For scavenging of HF using methoxytrimethylsilane, see: Hu, Y.; Porco, J. A., Jr. Tetrahedron Lett. 1998, $39,2711-2714$. - S 8 - 
temperature for $4 \mathrm{~h}$, filtered, and concentrated in vacuo. The crude product (mixture of "cis" and "trans" diastereomers) was purified by silica gel chromatography and dissolved in anhydrous $\mathrm{CH}_{2} \mathrm{Cl}_{2}$ $(10 \mathrm{~mL})$. To this solution was added anhydrous $\mathrm{HCl}(0.8 \mathrm{~mL}, 1.0 \mathrm{M}$ in ether $)$ at $0{ }^{\circ} \mathrm{C}$. The reaction mixture was stirred at $0{ }^{\circ} \mathrm{C}$ for $30 \mathrm{~min}$, then at $\mathrm{rt}$ for an additional $30 \mathrm{~min}$. Concentration in vacuo provided the pure "trans" diastereomer (28) (202 $\mathrm{mg}, 0.722 \mathrm{mmol}, 80 \%)$ as a colorless oil which was used without further purification. ${ }^{1} \mathrm{H} \mathrm{NMR}\left(400 \mathrm{MHz}, \mathrm{CDCl}_{3}\right): \delta 4.70(1 \mathrm{H}, \mathrm{d}, J=10.4), 4.35(1 \mathrm{H}, \mathrm{s})$, $3.50(2 \mathrm{H}, \mathrm{m}), 3.30(2 \mathrm{H}, \mathrm{m}), 2.89(1 \mathrm{H}, \mathrm{dd}, J=4.0,12.4), 2.56(2 \mathrm{H}, \mathrm{m}), 2.40(1 \mathrm{H}, \mathrm{m}), 2.06(1 \mathrm{H}, \mathrm{m})$, $1.86(2 \mathrm{H}, \mathrm{m}), 1.56(2 \mathrm{H}, \mathrm{m}), 1.12(3 \mathrm{H}, \mathrm{t}, J=7.2) \mathrm{ppm} ;{ }^{13} \mathrm{C} \mathrm{NMR}\left(75 \mathrm{MHz}, \mathrm{CDCl}_{3}\right): \delta 206.9,179.4$, $178.5,71.3,70.6,46.1,45.0,40.1,39.4,38.0,33.5,23.1,18.3,13.0$ ppm; HRMS (CI) $[\mathrm{M}+\mathrm{H}]^{+}$: calcd. for $\mathrm{C}_{14} \mathrm{H}_{20} \mathrm{NO}_{5} 282.1341$, found 282.1352. $[\alpha]_{\mathrm{D}}^{23}=+158^{\circ}\left(\mathrm{c}=0.20 \mathrm{CHCl}_{3}\right)$.

Scheme 7. Synthesis of angular epoxyquinol 29.
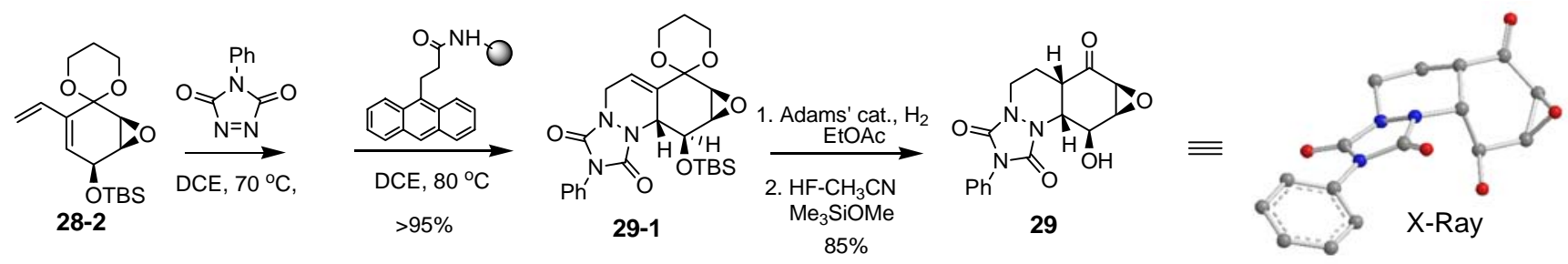

\section{8-Hydroxy-2-phenyl-hexahydro-7-oxa-2,3a,8b-triaza-cyclopenta[a]cyclopropa[g]naphthalene-}

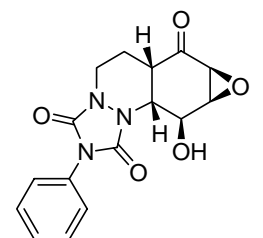

29

1,3,6-trione (29): Diene 28-2 (324 mg, $1.0 \mathrm{mmol}$ ) and $N$-phenyl triazolinedione (262 $\mathrm{mg}, 1.5 \mathrm{mmol}$ ) were placed in a Quest $2105 \mathrm{~mL}$ reaction vessel and dissolved in anhydrous toluene $(5 \mathrm{~mL})$. The reaction mixture was agitated at $70{ }^{\circ} \mathrm{C}$ for $6 \mathrm{~h}$ at which time 1.0 equiv. of the dienophile scavenger resin $(1 \mathrm{~g}, 1 \mathrm{mmol}, 1.08 \mathrm{mmol} / \mathrm{g})$ was added. The reaction mixture was agitated at $70{ }^{\circ} \mathrm{C}$ for an additional $4 \mathrm{~h}$, filtered, and concentrated in vacuo. To the residue (29-1) was added Adam's catalyst (20 w.t. \%), and distilled EtOAc $(5 \mathrm{~mL})$. The reaction mixture was stirred under a hydrogen atmosphere at $\mathrm{rt}$ for 48 $\mathrm{h}$, filtered, and concentrated in vacuo. The residue was dissolved in HPLC grade acetonitrile (10 mL) followed by the addition of $48 \%$ aq. HF $(1 \mathrm{~mL})$. The reaction mixture was stirred at $\mathrm{rt}$ for $24 \mathrm{~h}$ and quenched by addition of methoxytrimethylsilane $(0.23 \mathrm{~mL}, 1.67 \mathrm{mmol})$. After stirring at $\mathrm{rt}$ for $3 \mathrm{~h}$, the reaction mixture was concentrated in vacuo to provide 29 (260 mg, $0.80 \mathrm{mmol}, 85 \%$ from 29-1). X-ray crystal structure analysis was used to confirm the stereochemistry of 29. m.p. 123.0-124.0 ${ }^{\circ} \mathrm{C}$; ${ }^{1} \mathrm{H}$ NMR $\left(400 \mathrm{MHz}, \mathrm{C}_{6} \mathrm{D}_{6}\right): \delta 7.45(5 \mathrm{H}, \mathrm{m}), 4.90(1 \mathrm{H}, \mathrm{dd}, J=5.6,10.4), 4.63(1 \mathrm{H}, \mathrm{t}, J=10.0), 4.15(1 \mathrm{H}, \mathrm{m})$, $3.80(1 \mathrm{H}, \mathrm{d}, J=4.0), 3.50(1 \mathrm{H}, \mathrm{d}, J=4.0), 3.21(1 \mathrm{H}, \mathrm{m}), 2.82(1 \mathrm{H}, \mathrm{m}), 2.68(1 \mathrm{H}, \mathrm{d}, J=10.4), 2.16$ $(1 \mathrm{H}, \mathrm{m}), 1.83(1 \mathrm{H}, \mathrm{m}) \mathrm{ppm} ;{ }^{13} \mathrm{C} \mathrm{NMR}\left(75 \mathrm{MHz}, \mathrm{CDCl}_{3}\right): \delta 201.5,153.5,152.5,131.0,129.2,128.4$, 125.6, 65.8, 57.6, 55.9, 51.9, 44.2, 43.5, 24.2 ppm; HRMS (CI) $[\mathrm{M}+\mathrm{H}]^{+}$: calcd. for $\mathrm{C}_{16} \mathrm{H}_{16} \mathrm{~N}_{3} \mathrm{O}_{5}$ 330.1091, found 330.1037. $[\alpha]_{\mathrm{D}}^{23}=-20^{\circ}\left(\mathrm{c}=0.10 \mathrm{CHCl}_{3}\right)$. 
Scheme 8. Synthesis of 30.
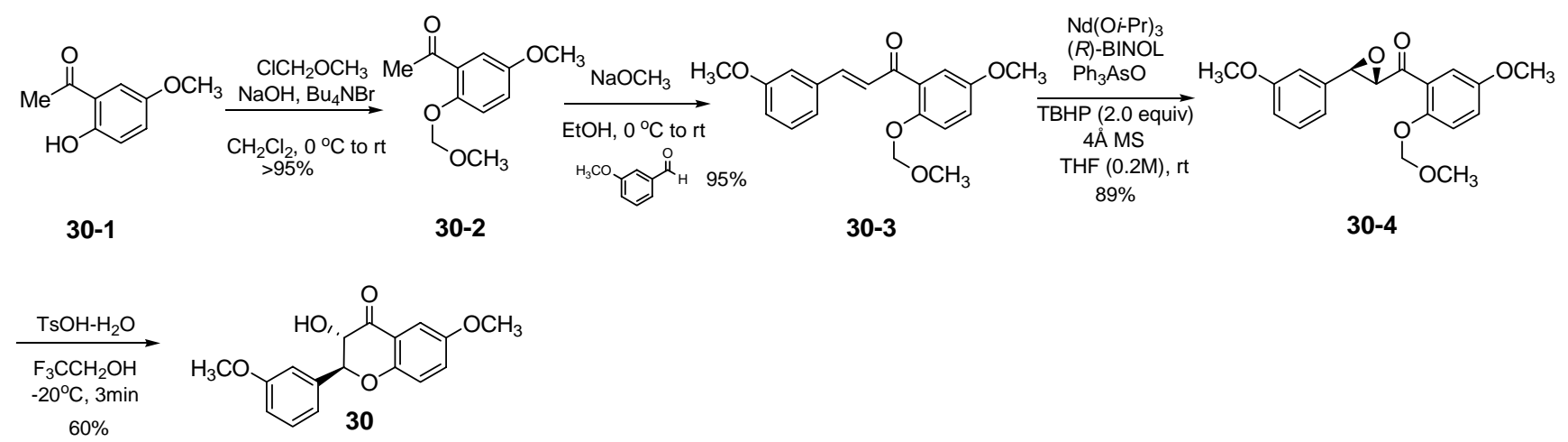

(2S,3S)-3-Hydroxy-6-methoxy-2-(3-methoxyphenyl)chroman-4-one (30): A $500 \mathrm{~mL}$ round bottom

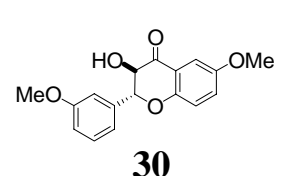
flask was charged with 1-(2-hydroxy-5-methoxyphenyl)ethanone (30-1) (15.33 g, $92.3 \mathrm{mmol}$ ). The substrate was dissolved in sodium hydroxide (4M aq. solution, $100 \mathrm{~mL})$ and methylene chloride $(100 \mathrm{~mL})$ and stirred at room temperature for 30 minutes. tetra- $N$-butylammonium bromide $(7.34 \mathrm{~g}, 22.8 \mathrm{mmol})$ and chloromethyl methyl ether $(11.0 \mathrm{~mL}, 14.5 \mathrm{~mol})$ were added and the solution stirred for $12 \mathrm{~h}$ at room temperature. The reaction was diluted with water $(200 \mathrm{~mL})$ and the organic layer was extracted with dichloromethane $(3 \times 150 \mathrm{~mL})$. The combined organic layers were washed with saturated sodium chloride, dried with sodium sulfate, filtered, and concentrated under reduced pressure. The crude oil was purified through a silica gel plug $(150 \mathrm{~mL})$ using a 1:1 mixture of hexane:ethyl acetate $(600 \mathrm{~mL})$ as eluant to yield 30-2 (19.31 g, $92.0 \mathrm{mmol})$ as a clear oil which was used without further purification. A $100 \mathrm{~mL}$ round bottom flask was charged with 3-methoxy-benzaldehyde $(3.00 \mathrm{~mL}, 24.6 \mathrm{mmol})$ and 30-2 (3.81 g, $18.1 \mathrm{mmol})$. The reagents were dissolved in ethanol $(20.0 \mathrm{~mL})$ and the solution cooled to $0{ }^{\circ} \mathrm{C}$. Sodium methoxide $(2.26 \mathrm{~g}, 41.9 \mathrm{mmol})$ was added and the solution was stirred from $0{ }^{\circ} \mathrm{C}$ to $\mathrm{rt}$ over 12 hours. The reaction was quenched with water $(40 \mathrm{~mL})$ and the organic layer was extracted with ethyl acetate $(3 \times 30 \mathrm{~mL})$. The combined organic layers were washed with saturated sodium chloride $(150 \mathrm{~mL})$, dried with sodium sulfate, filtered, and concentrated under reduced pressure. The crude product mixture was further purified by flash column chromatography over silica gel (20:1 to 1:1 hexane:ethyl acetate) to afford 30-3 $(5.64 \mathrm{~g}, 17.2 \mathrm{mmol})$ as a yellow oil. A $100 \mathrm{~mL}$ round bottom flask was charged with neodymium isopropoxide $(236 \mathrm{mg}, 0.73 \mathrm{mmol})$ and $(R)-(+)-1,1$ '-binaphthalene-2,2'diol $(210 \mathrm{mg}, 0.73 \mathrm{~mol})$. The reagents were dissolved in dry tetrahydrofuran $(27.5 \mathrm{~mL})$ and stirred at room temperature for $1 \mathrm{~h}^{11}$ Triphenylarsine oxide $(238 \mathrm{mg}, 0.73 \mathrm{mmol})$, and $4 \AA$ molecular sieves (4.87 $\mathrm{g}, 1 \mathrm{~g} / \mathrm{mmol}$ substrate) were added and the solution was stirred at room temperature for 1 hour. tert-Butyl hydroperoxide in $n$-decane $(5.0 \mathrm{M}, 1.9 \mathrm{~mL})$ was added and the solution was stirred at room temperature for 30 minutes. (E)-1-(5-Methoxy-2-(methoxymethoxy)phenyl)-3-(3methoxyphenyl)prop-2-en-1-one (30-3) (1.60 g, $4.87 \mathrm{mmol})$ was added and the solution was stirred at room temperature for 24 hours. The reaction was quenched by addition of $50 \mathrm{~mL}$ of a $2.5 \%$ citric acid solution. The organic layer was extracted with ethyl acetate $(3 \times 50 \mathrm{~mL})$, washed with saturated sodium chloride $(250 \mathrm{~mL})$, dried with sodium sulfate, filtered, and concentrated under reduced pressure. The crude product was further purified by flash column chromatography over silica gel (ethyl acetate:hexane, gradient of $=1: 20$ to $1: 4$ ) to yield the desired epoxide 30-4 (1.49 $\mathrm{g}, 4.32 \mathrm{mmol})$ as colorless oil. A $250 \mathrm{~mL}$ round bottom flask was charged with (5-methoxy-2(methoxymethoxy)phenyl)((2S,3R)-3-(3-methoxyphenyl)oxiran-2-yl)methanone (30-4) (760 mg, 2.208 $\mathrm{mmol})$. The substrate was dissolved in 2,2,2-trifluoroethanol $(100 \mathrm{~mL})$ and the solution was cooled to -

${ }^{11}$ Nemoto, T.; Ohshima, T.; Yamaguchi, K.; Shibasaki, M. J. Am. Chem. Soc. 2001, 123, 2725-2732. 
$20{ }^{\circ} \mathrm{C}$ in a water-IPA-dry ice bath. ${ }^{12}$ The solution was stirred at $-20{ }^{\circ} \mathrm{C}$ for 15 minutes. $p$ Toluenesulfonic acid monohydrate $(320.2 \mathrm{mg}, 1.683 \mathrm{mmol})$ was added. The solution stirred at $-20{ }^{\circ} \mathrm{C}$ for 3 minutes. The reaction was quenched by the addition of water $(100 \mathrm{~mL})$ and the organic layer was extracted with dichloromethane $(3 \times 100 \mathrm{~mL})$. The combined organic layers were washed with saturated sodium chloride $(350 \mathrm{~mL})$, dried with sodium sulfate, filtered, and concentrated under reduced pressure. The crude product was further purified by flash column chromatography over silica gel (hexane:ethyl acetate, gradient of 20:3 to 1:1) to afford the desired product $\mathbf{3 0}$ as a white solid (400 $\mathrm{mg}, 1.32 \mathrm{mmol}$ ) in 93\% ee by chiral HPLC analysis (Chiralcel OD-H, 85:15 hexanes:IPA, $1 \mathrm{~mL} / \mathrm{min}$, $254 \mathrm{~nm}, t_{\mathrm{R}}($ minor $)=10.50 \mathrm{~min}, t_{\mathrm{R}}($ major $\left.)=12.27 \mathrm{~min}\right)$; m.p. $119.0-120.5{ }^{\circ} \mathrm{C} ;{ }^{1} \mathrm{H} \mathrm{NMR}(400 \mathrm{MHz}$, $\left.\mathrm{CDCl}_{3}\right) \delta 7.38(\mathrm{t}, 1 \mathrm{H}, J=8.0 \mathrm{~Hz}), 7.32(\mathrm{~d}, 1 \mathrm{H}, J=3.6 \mathrm{~Hz}), 7.15(\mathrm{~m}, 3 \mathrm{H}), 6.97(\mathrm{~m}, 2 \mathrm{H}), 5.08(\mathrm{~d}, 1 \mathrm{H}, J$ $=12.4 \mathrm{~Hz}), 4.61(\mathrm{~d}, 1 \mathrm{H}, J=12.4 \mathrm{~Hz}), 3.85(\mathrm{~s}, 3 \mathrm{H}), 3.83(\mathrm{~s}, 3 \mathrm{H}), 3.72(\mathrm{~s}, 1 \mathrm{H}) ;{ }^{13} \mathrm{C} \mathrm{NMR}(100 \mathrm{MHz}$, $\left.\mathrm{CDCl}_{3}\right) \delta 159.9,156.5,154.7,138.0,129.9,126.3,120.0,119.6,118.4,114.9,113.2,107.5,84.2,73.9$, 56.0, 55.4; IR (thin film) vmax 3462, 3084, 3063, 3036, 3003, 2961, 2924, 2894, 2841, 1692, 1658 , 1602, 1490, 1466, 1344, 1322, $1219 \mathrm{~cm}^{-1}$; $\operatorname{HRMS~(m/z)~(EI)~}[\mathrm{M}]^{+}$calculated for $\mathrm{C}_{17} \mathrm{H}_{16} \mathrm{O}_{5}: 300.0998$, found: $300.0987 ;[\alpha]_{\mathrm{D}}^{23}=-14.7^{\circ}\left(\mathrm{c}=0.865, \mathrm{CH}_{2} \mathrm{Cl}_{2}\right)$.

Scheme 9. Synthesis of 31.

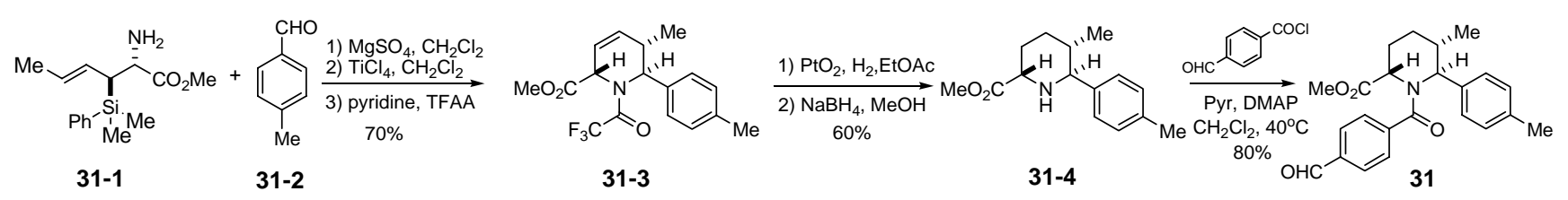

1-(4-Formyl-benzoyl)-5-methyl-6-p-tolyl-piperidine-2-carboxylic acid methyl ester (31): To a

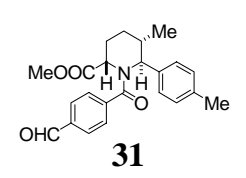
round bottom flask was added 2-amino-3-(dimethyl-phenyl-silanyl)-hex-4-enoic acid methyl ester $^{13}$ (31-1) (1000 mg, $\left.3.61 \mathrm{mmol}\right)$, 4-methylbenzaldehyde (31-2) (0.467 mL, $3.96 \mathrm{mmol}$ ), magnesium sulfate $(870 \mathrm{mg}, 7.2 \mathrm{mmol})$, and $\mathrm{CH}_{2} \mathrm{Cl}_{2}(10$ $\mathrm{mL})$. The mixture was stirred at $\mathrm{rt}$ for $8 \mathrm{~h}$ then filtered through a Celite plug. The solution was concentrated and the crude imine was dissolved in $\mathrm{CH}_{2} \mathrm{Cl}_{2}$. The reaction was cooled to $-78{ }^{\circ} \mathrm{C}$ and titanium tetrachloride $\left(1 \mathrm{M} \mathrm{CH}_{2} \mathrm{Cl}_{2}\right.$ solution, $\left.12 \mathrm{~mL}, 12 \mathrm{mmol}\right)$ was added dropwise. The reaction was stirred overnight $\left(-78{ }^{\circ} \mathrm{C}-\mathrm{rt}\right)$, cooled to $-78{ }^{\circ} \mathrm{C}$, and quenched by addition of sat. $\mathrm{NaHCO}_{3}$. The frozen solid was warmed to $\mathrm{rt}$ during $\sim 1 \mathrm{~h}$, transferred to a separation funnel, and extracted with $\mathrm{CH}_{2} \mathrm{Cl}_{2}(3 \mathrm{x})$. The combined $\mathrm{CH}_{2} \mathrm{Cl}_{2}$ extract were dried over $\mathrm{MgSO}_{4}$, filtered, and concentrated. The residue was dissolved in $\mathrm{CH}_{2} \mathrm{Cl}_{2}(3 \mathrm{~mL})$ and the solution was cooled to $-78{ }^{\circ} \mathrm{C}$ followed by addition of pyridine $(0.73 \mathrm{~mL}, 9 \mathrm{mmol})$ and trifluoroacetic anhydride $(0.56 \mathrm{~mL}, 3.96 \mathrm{~mol})$. The solution was stirred at $-78{ }^{\circ} \mathrm{C}$ for $2 \mathrm{~h}$ and quenched by addition of water. The aqueous phase was extracted with $\mathrm{CH}_{2} \mathrm{Cl}_{2}(3 \mathrm{x})$, and the combined organic solution was dried over $\mathrm{MgSO}_{4}$, concentrated, and chromatographed (0-40\% EtOAc in Hex) to afford 31-3. To a 1-neck round-bottom flask was added 31-3 (0.79 g, $1.95 \mathrm{mmol})$, ethyl acetate $(10 \mathrm{~mL})$, and Adam's catalyst $(90 \mathrm{mg})$. The mixture was purged $3 \mathrm{X}$ with $\mathrm{H}_{2}$ and then stirred under a $\mathrm{H}_{2}$ atmosphere. After $3 \mathrm{~h}$, the mixture was filtered through Celite and washed with EtOAc. The combined solution was concentrated in vacuo and dried in vacuo. The crude product was directly transferred to a flask followed by the addition of methanol $(5 \mathrm{~mL})$. After the solution was cooled to $0{ }^{\circ} \mathrm{C}$, sodium tetrahydroborate $(151 \mathrm{mg}, 4.0 \mathrm{mmol})$ was added in one portion. Vigorous bubbling was observed. After $5 \mathrm{~h}$, sat. $\mathrm{NaHCO}_{3}$ (aq.) was added to quench the reaction, and the aqueous layer was extracted with $\mathrm{CH}_{2} \mathrm{Cl}_{2}(3 \mathrm{x})$. Silica gel chromatography $(0-50 \%$

\footnotetext{
${ }^{12}$ Augustyn, J. A. N.; Bezuidenhoudt, B. C. B.; Ferreira, D. Tetrahedron 1990, 46, 2651-2660.

${ }^{13}$ Huang, H.; Spande, T. F.; Panek, J. S. J. Am. Chem. Soc. 2003, 125, 626.
} 
EtOAc in hexane) provided 31-4 (288 mg, $1.17 \mathrm{mmol}, 60 \%)$ as a colorless oil. (2S,5S,6S)-methyl 5methyl-6-p-tolylpiperidine-2-carboxylate (31-4) (151.0 mg, $0.611 \mathrm{mmol})$, 4-formylbenzoyl chloride (205.8 $\mathrm{mg}, 1.221 \mathrm{mmol})$, 4-dimethylaminopyridine $(7.5 \mathrm{mg}, 0.061 \mathrm{mmol})$, and methylene chloride $(3.00 \mathrm{~mL})$ was added to a high pressure tube. Pyridine $(123.4 \mu \mathrm{L}, 1.526 \mathrm{mmol})$ was added at $\mathrm{rt}$ and then the reaction mixture was heated at $40{ }^{\circ} \mathrm{C}$ for $12 \mathrm{~h}$. The reaction was cooled to $\mathrm{rt}$, washed with sat. $\mathrm{NaHCO}_{3}$, extracted with additional $\mathrm{CH}_{2} \mathrm{Cl}_{2}(2 \times 15 \mathrm{~mL})$, concentrated, and chromatographed $(0-50 \%$ EtOAc/hexane) to afford the desired product 31 as a colorless oil (185 mg, $0.489 \mathrm{mmol}, 80 \%) .{ }^{1} \mathrm{H}$ NMR $\left(400 \mathrm{MHz}, \mathrm{CDCl}_{3}\right) \delta 9.95(\mathrm{~s}, 1 \mathrm{H}), 7.75(\mathrm{~d}, 2 \mathrm{H}, J=7.6 \mathrm{~Hz}), 7.47(\mathrm{~d}, 2 \mathrm{H}, J=7.2 \mathrm{~Hz}), 7.08(\mathrm{~s}$, 4H), $4.54(\mathrm{bs}, 1 \mathrm{H}), 4.46(\mathrm{dd}, 1 \mathrm{H}, J=5.2,8.0 \mathrm{~Hz}), 3.77(\mathrm{~s}, 3 \mathrm{H}), 2.30(\mathrm{~s}, 3 \mathrm{H}), 2.25(\mathrm{~m}, 2 \mathrm{H}), 1.92(\mathrm{~m}$, $1 \mathrm{H}), 1.72(\mathrm{~m}, 1 \mathrm{H}), 1.38(\mathrm{~m}, 1 \mathrm{H}), 1.04(\mathrm{~d}, 3 \mathrm{H}, J=6.8 \mathrm{~Hz}) ;{ }^{13} \mathrm{C} \mathrm{NMR}\left(100 \mathrm{MHz}, \mathrm{CDCl}_{3}\right) \delta 191.7$, $173.5,171.9,142.1,137.9,136.9,129.7,129.6,127.6,126.5,65.1,55.8,52.5,34.7,25.8,22.8,21.0$, 18.9; IR (thin film) vmax 2952, 2871, 1743, 1702, 1641, 1542, 1456, 1382, 1203, $843 \mathrm{~cm}^{-1}$; CIHRMS $[\mathrm{M}+\mathrm{H}]^{+}$calculated for $\mathrm{C}_{23} \mathrm{H}_{26} \mathrm{NO}_{4}: 380.1862$, found: $380.1802 ;[\alpha]_{\mathrm{D}}^{23}=-182.9^{\circ}\left(\mathrm{c}=0.555, \mathrm{CH}_{2} \mathrm{Cl}_{2}\right)$.

\subsection{Synthesis of $N$-alkoxyphthlimides. ${ }^{14}$}

2-((2S,3R)-2,4-Dimethyl-3-(phenoxymethyl)pentyloxy)isoindoline-1,3-dione (Phth-6): To a roundbottom flask was added (2S,3R)-2,4-dimethyl-3-(phenoxymethyl)pentan-1-ol (41) $(150.0 \mathrm{mg}, \quad 0.675 \mathrm{mmol}), \quad N$-hydroxyphthalimide $(143.1 \mathrm{mg}, \quad 0.877 \mathrm{mmol})$, Me tiphenylphosphine $(230.0 \mathrm{mg}, 0.877 \mathrm{mmol})$ and tetrahydrofuran $(5.00 \mathrm{~mL})$. The
Me Me ${ }^{\circ}$. Th Me tiphenylphosphine $(230.0 \mathrm{mg}, 0.877 \mathrm{mmol})$ and tetrahydrofuran $(5.00 \mathrm{~mL})$. The
Me Me ${ }^{\circ}$. Th

Phth-6 was added dropwise. The reaction mixture was stirred for $5 \mathrm{~h}\left(0{ }^{\circ} \mathrm{C}\right.$ to rt $)$, concentrated and then chromatographed (0-40\% EtOAc/Hexane) to afford the desired product Phth-6 as colorless oil (230 mg, $0.624 \mathrm{mmol}, 95 \%) .{ }^{1} \mathrm{H}$ NMR (400 MHz, $\left.\mathrm{CDCl}_{3}\right) \delta 7.84(\mathrm{~m}, 2 \mathrm{H}), 7.75(\mathrm{~m}, 2 \mathrm{H}), 7.41(\mathrm{~m}$, 2H), $7.33(\mathrm{~m}, 2 \mathrm{H}), 7.26(\mathrm{~m}, 1 \mathrm{H}), 4.84(\mathrm{~d}, 1 \mathrm{H}, J=11.6 \mathrm{~Hz}), 4.72(\mathrm{~d}, 1 \mathrm{H}, J=10.8 \mathrm{~Hz}), 4.29(\mathrm{t}, 1 \mathrm{H}, J=$ $8.0 \mathrm{~Hz}), 4.07(\mathrm{dd}, 1 \mathrm{H}, J=5.6,8.4 \mathrm{~Hz}), 3.50(\mathrm{dd}, 1 \mathrm{H}, J=2.8,8.4 \mathrm{~Hz}), 2.32(\mathrm{~m}, 1 \mathrm{H}), 1.96(\mathrm{~m}, 1 \mathrm{H})$, $1.09(\mathrm{~d}, 3 \mathrm{H}, J=6.8 \mathrm{~Hz}), 1.02(\mathrm{~d}, 3 \mathrm{H}, J=7.6 \mathrm{~Hz}), 1.09(\mathrm{~d}, 3 \mathrm{H}, J=7.6 \mathrm{~Hz}) ;{ }^{13} \mathrm{C} \mathrm{NMR}(100 \mathrm{MHz}$, $\left.\mathrm{CDCl}_{3}\right) \delta 163.8,139.5,134.7,129.2,127.7,127.5,123.7,84.3,81.4,75.2,35.0,31.4,19.9,19.8,10.5$; IR (thin film) vmax 3031, 2964, 2876, 1789, 1733, 1468, 1372, 1187, 1127, 994, 877, $700 \mathrm{~cm}^{-1}$; CIHRMS $[\mathrm{M}]^{+}$calculated for $\mathrm{C}_{22} \mathrm{H}_{25} \mathrm{NO}_{4}$ : 367.1784, found: $367.1841 ;[\alpha]_{\mathrm{D}}{ }^{23}=-24.9^{\circ}(\mathrm{c}=0.609$, $\mathrm{CH}_{2} \mathrm{Cl}_{2}$ ).

2-(((2R,3S,6S)-3-(4-Methoxyphenoxy)-3,6-dihydro-6-phenyl-2H-pyran-2-yl)methoxy) isoindoline1,3-dione (Phth-7): To a round bottom flask was added [3-(4-methoxy-phenoxy)-6-

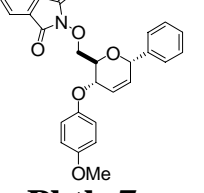
phenyl-3,6-dihydro-2H-pyran-2-yl]-methanol (44) (210.8 mg, $0.675 \mathrm{mmol}$ ), pyridine $(109.1 \mu \mathrm{L}, 1.349 \mathrm{mmol})$, and methylene chloride $(2.00 \mathrm{~mL})$. The solution was cooled to 0 ${ }^{\circ} \mathrm{C}$ and trifluoromethanesulfonic anhydride $(170.3 \mu \mathrm{L}, 1.012 \mathrm{mmol})$ was added dropwise. The reaction mixture was stirred for $1 \mathrm{~h}$ at $0{ }^{\circ} \mathrm{C}$, eluted through a silica gel plug $(5 \mathrm{~g})$

Phth-7 using EtOAc and concentrated in vacuo. $N$-hydroxyphthalimide $(143.1 \mathrm{mg}, 0.877 \mathrm{mmol})$ and acetonitrile $(2.00 \mathrm{~mL})$ were added and the mixture was cooled to $0{ }^{\circ} \mathrm{C}$. 1,8 diazabicyclo[5.4.0] undec-7-ene $(131.2 \mu \mathrm{L}, 0.877 \mathrm{mmol})$ was added dropwise and the reaction was stirred for $1 \mathrm{~h}\left(0{ }^{\circ} \mathrm{C}-\mathrm{rt}\right)$. The reaction mixture was concentrated and was chromatographed $(0-30 \%$ EtOAc/Hexane) to afford the desired product Phth-7 as a pale yellow solid $(215 \mathrm{mg}, 0.473 \mathrm{mmol}$, 70\%). m. p. $125.5-126.0{ }^{\circ} \mathrm{C} ;{ }^{1} \mathrm{H}$ NMR (400 MHz, $\left.\mathrm{CDCl}_{3}\right) \delta 7.77(\mathrm{~m}, 2 \mathrm{H}), 7.70(\mathrm{~m}, 2 \mathrm{H}), 7.38(\mathrm{~m}, 2 \mathrm{H})$,

\footnotetext{
${ }^{14} \mathrm{~N}$-alkoxyphthalimides are named according to the assigned numbers of corresponding alkoxyamine monomers. For example, Phth-6 is a phthalimide precursor to alkoxyamine 6.
} 
$7.33(\mathrm{~m}, 3 \mathrm{H}), 7.01(\mathrm{~d}, 1 \mathrm{H}, J=8.4 \mathrm{~Hz}), 6.86(\mathrm{~d}, 1 \mathrm{H}, J=8.8 \mathrm{~Hz}), 6.21(\mathrm{~d}, 1 \mathrm{H}, J=11.2 \mathrm{~Hz}), 4.72(\mathrm{dd}$, $1 \mathrm{H}, J=1.2 \mathrm{~Hz}, 10.4 \mathrm{~Hz}), 5.33$ (br s, 1h), $5.10(\mathrm{~d}, 1 \mathrm{H}, J=8.0 \mathrm{~Hz}), 4.05(\mathrm{~m}, 1 \mathrm{H}), 3.77(\mathrm{~s}, 3 \mathrm{H}) ;{ }^{13} \mathrm{C}$ NMR $\left(100 \mathrm{MHz}, \mathrm{CDCl}_{3}\right) \delta 163.3,154.6,151.3,138.9,134.5,130.4,129.0,128.6,128.3,125.8$, 123.6, 76.7, 74.5, 70.2, 69.2, 55.8; IR (thin film) vmax 3061, 2931, 1789, 1732, 1505, 1374, 1225, 1187, 1032, 876, $827 \mathrm{~cm}^{-1}$; CIHRMS [M] ${ }^{+}$calculated for $\mathrm{C}_{27} \mathrm{H}_{23} \mathrm{NO}_{6}$ : 457.1525, found: 457.1596; $[\alpha]_{\mathrm{D}}^{23}=23.9^{\mathrm{o}}\left(\mathrm{c}=0.364, \mathrm{CH}_{2} \mathrm{Cl}_{2}\right)$.

Scheme 10. Synthesis of Phth-8.
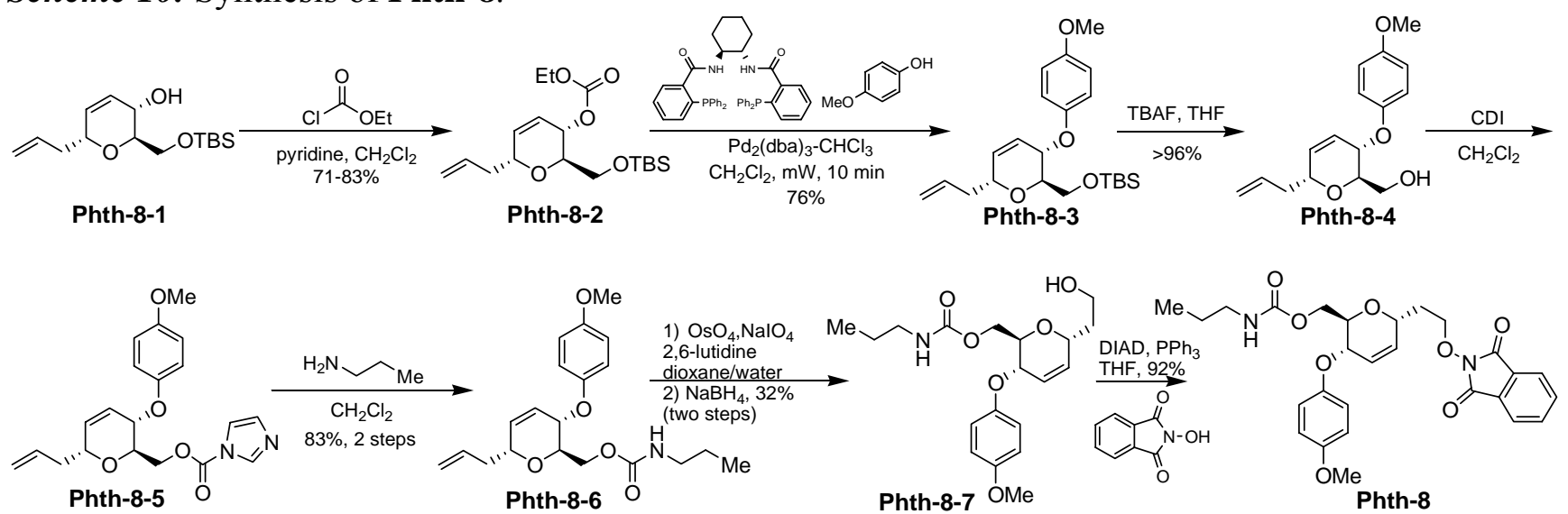

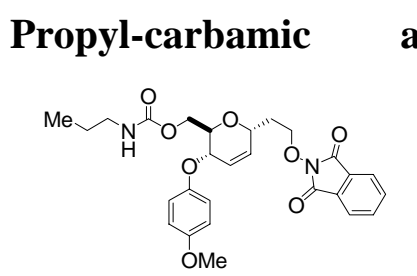

Phth-8

acid 6-[2-(1,3-dioxo-1,3-dihydro-isoindol-2-yloxy)-ethyl]-3-(4-methoxyphenoxy)-3,6-dihydro-2H-pyran-2-ylmethyl ester (Phth-8): Phth-8-1 (3.45 g, $12.1 \mathrm{mmol})$ was dissolved in dichloromethane under an argon atmosphere. Pyridine $(2.94 \mathrm{~mL}, 36.4 \mathrm{mmol})$ was added, the reaction was cooled to $0{ }^{\circ} \mathrm{C}$, and ethyl chloroformate $(2.90 \mathrm{~mL}, 30.4 \mathrm{mmol})$ was added dropwise. The reaction was stirred for 40 minutes, diluted with dichloromethane, and washed with sat. sodium bicarbonate $(2 \mathrm{x})$ and brine

(2x). The organic layers were dried with sodium sulfate, concentrated, and chromatographed over silica gel using 5\% ethyl acetate/hexanes to afford compound Phth-8-2 as a colorless oil. Phth-8-2 (2.0 g, $5.60 \mathrm{mmol})$, tris(dibenzylidene acetone) dipalladium (0) chloroform adduct (145 $\mathrm{mg}, 0.14$ $\mathrm{mmol})$, and $(1 S, 2 S)$-(-)-1,2-diaminocyclohexane- $N, N^{\prime}$-bis (2'-diphenylphosphinobenzoyl) (194 mg, $0.28 \mathrm{mmol})$ were added to a flame-dried microwave vial $(80 \mathrm{~mL})$ and then dissolved in degassed dichloromethane $(20 \mathrm{~mL})$ under an argon atmosphere. After 30 minutes, $p$-methoxyphenol $(1.04 \mathrm{~g}$, $8.41 \mathrm{mmol})$ was added, and the reaction was microwaved at $100{ }^{\circ} \mathrm{C}$ for 10 minutes $(150 \mathrm{~W})$. The solution was concentrated onto silica gel and chromatographed on silica gel using 2-3\% ethyl acetate/ hexanes to yield Phth-8-3 as colorless oil. Phth-8-3 (1.72 g, $4.40 \mathrm{mmol})$ was dissolved in tetrahydrofuran under an argon atmosphere and a solution of $1 \mathrm{M}$ tetrabutylammonium fluoride in tetrahydrofuran $(5.3 \mathrm{~mL}, 5.28 \mathrm{mmol})$ was added. After $1 \mathrm{~h}$, the solution was concentrated and chromatographed over silica gel using 10-20\% ethyl acetate/hexanes to yield Phth-8-4 as a colorless oil. Phth-8-4 (948 mg, $3.43 \mathrm{mmol}$ ) was dissolved in dichloromethane under an argon atmosphere, and $N, N$-carbonyldiimidazole $(611 \mathrm{mg}, 3.77 \mathrm{mmol})$ was added. After 38 minutes, the reaction was concentrated to provide crude substrate Phth-8-5. Phth-8-5 (1.27 g, $3.43 \mathrm{mmol})$ was dissolved in dichloromethane under an argon atmosphere and propylamine $(2.26 \mathrm{~mL}, 27.4 \mathrm{mmol})$ was added dropwise. After $1 \mathrm{~h}$, the solution was diluted with dichloromethane, washed with sodium bicarbonate $(1 \mathrm{x})$, and with brine $(1 \mathrm{x})$. The organic layer was dried with sodium sulfate, concentrated, and chromatographed on silica gel using 3-20\% ethyl acetate/hexanes to provide Phth-8-6. Phth-8-6 (600 $\mathrm{mg}, 1.66 \mathrm{mmol})$ was dissolved in dioxane-water $(3: 1,16 \mathrm{~mL})$. 2, 6-lutidine $(0.38 \mathrm{~mL}, 3.32 \mathrm{mmol})$ was added followed by addition of a $0.02 \mathrm{M}$ solution of osmium tetraoxide in toluene (1.66 $\mathrm{mL}, 0.03 \mathrm{mmol})$ 
and sodium periodate $(1.42 \mathrm{~g}, 6.64 \mathrm{mmol})$. After $2 \mathrm{~h}$, the reaction was diluted with water and extracted with dichloromethane $(3 \mathrm{x})$. The organic layer was washed with brine $(1 \mathrm{x})$, dried with sodium sulfate, and concentrated to yield the crude aldehyde. The aldehyde (107 $\mathrm{mg}, 0.29 \mathrm{mmol})$ was dissolved in methanol under an argon atmosphere, cooled to $0{ }^{\circ} \mathrm{C}$, and sodium borohydride $(22 \mathrm{mg}, 0.59 \mathrm{mmol})$ was added. After 10 minutes, the reaction was warmed to room temperature and stirred for $100 \mathrm{~min}$ (monitored by ${ }^{1} \mathrm{H} \mathrm{NMR}$ ). The solution was concentrated and chromatographed over silica gel using $2 \%$ methanol/ dichloromethane to yield Phth-8-7 as a colorless oil. To the reaction flask was added propyl-carbamic acid 6-(2-hydroxy-ethyl)-3-(4-methoxy-phenoxy)-3,6-dihydro-2H-pyran-2-ylmethyl ester (160.0 mg, $0.438 \mathrm{mmol})$ (Phth-8-7), $N$-hydroxyphthalimide (92.9 $\mathrm{mg}, 0.569$ mmol), triphenylphosphine $(149.3 \mathrm{mg}, 0.569 \mathrm{mmol})$ and tetrahydrofuran $(5.00 \mathrm{~mL})$. The solution was cooled to $0{ }^{\circ} \mathrm{C}$ and diisopropyl azodicarboxylate $(112.1 \mu \mathrm{L}, 0.569 \mathrm{mmol})$ was added dropwise. The reaction mixture was stirred for $5 \mathrm{~h}\left(0{ }^{\circ} \mathrm{C}-\mathrm{rt}\right)$, concentrated and then chromatographed $(0-80 \%$ EtOAc/Hexane $)$ to afford the desired product (Phth-8) as a white solid (205 mg, 0.403 mmol, 92\%). m. p. 92.0-94.0 ${ }^{\circ} \mathrm{C} ;{ }^{1} \mathrm{H}$ NMR $\left(400 \mathrm{MHz}, \mathrm{CDCl}_{3}\right) \delta 7.79(\mathrm{~m}, 2 \mathrm{H}), 7.75(\mathrm{~m}, 2 \mathrm{H}), 6.80(\mathrm{~m}, 4 \mathrm{H}), 5.94(\mathrm{~m}, 2 \mathrm{H}), 5.28$ (br s, $1 \mathrm{H}), 4.55(\mathrm{~d}, 1 \mathrm{H}, J=9.2 \mathrm{~Hz}), 4.49(\mathrm{~d}, 1 \mathrm{H}, J=6.8 \mathrm{~Hz}), 4.39(\mathrm{~m}, 3 \mathrm{H}), 4.15(\mathrm{~m}, 1 \mathrm{H}), 3.96(\mathrm{~m}, 1 \mathrm{H}), 3.74$ (s, 3H), $3.13(\mathrm{dd}, 2 \mathrm{H}, J=6.0 \mathrm{~Hz}, 12.8 \mathrm{~Hz}), 2.17(\mathrm{~m}, 1 \mathrm{H}), 2.04(\mathrm{~m}, 1 \mathrm{H}), 1.54(\mathrm{dd}, 2 \mathrm{H}, J=6.4 \mathrm{~Hz}, 13.2$ $\mathrm{Hz}), 0.92(\mathrm{t}, 3 \mathrm{H}, J=6.8 \mathrm{~Hz}) ;{ }^{13} \mathrm{C}$ NMR $\left(100 \mathrm{MHz}, \mathrm{CDCl}_{3}\right) \delta 164.0,156.7,154.6,151.3,134.9,132.1$, $129.0,125.2,123.8,117.4,115.0,75.6,70.3,69.9,69.3,64.0,55.9,43.1,31.4,23.3$, 11.5; IR (thin film) vmax 3339, 3105, 3043, 2962, 2835, 1857, 1789, 1715, 1692, 1611, 1538, 1504, 1461, 1380, 1266, 1220, 1134, 1035, 888, 830, $783 \mathrm{~cm}^{-1}$; CIHRMS [M] calculated for $\mathrm{C}_{27} \mathrm{H}_{30} \mathrm{~N}_{2} \mathrm{O}_{8}: 511.2002$, found: $511.2099 ;[\alpha]_{\mathrm{D}}^{23}=79.5^{\circ}\left(\mathrm{c}=0.391, \mathrm{CH}_{2} \mathrm{Cl}_{2}\right)$.

Scheme 11. Synthesis of Phth-9.

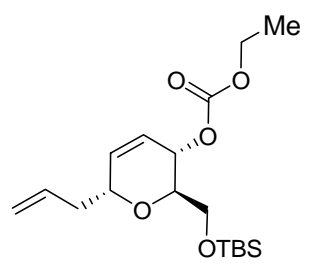

Phth-8-2

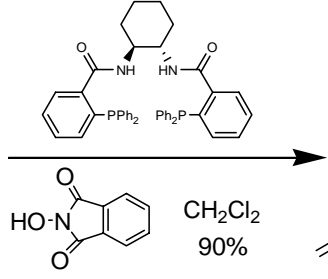

\%

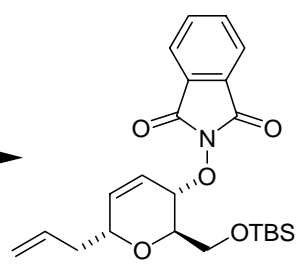

Phth-9-1

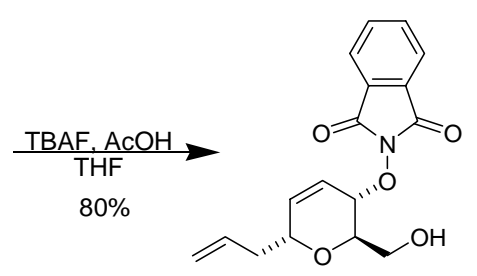

Phth-9

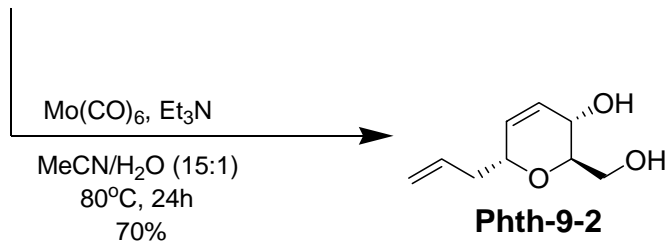

2-((2R,3S,6R) - 6- Allyl - 2- hydroxymethyl - 3,6 - dihydro - 2H- pyran - 3-yloxy) - isoindole - 1,3dione (Phth-9): Carbonic acid 6-allyl-2-(tert-butyl-dimethyl-silanyloxymethyl)-3,6-

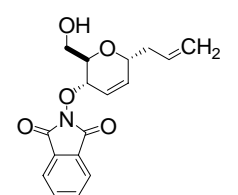

Phth-9 dihydro-2H-pyran-3-yl ester ethyl ester (Phth-8-2) (100 mg, $0.3 \mathrm{mmol})$ was dissolved in degassed methylene chloride $(2 \mathrm{~mL})$ under an atmosphere of argon. $(1 S, 2 S) \quad-(-)-1,2$-diaminocyclohexane- $N, N^{\prime}$-bis (2'-diphenylphosphinobenzoyl) (4.8 $\mathrm{mg}, 0.0070 \mathrm{mmol})$ and tris-(dibenzylideneacetone) dipalladium(0) chloroform adduct $(10 \mathrm{mg}, 0.010 \mathrm{~mol})$ were added to the reaction. After stirring for 30 minutes at $\mathrm{rt}, N$-hydroxyphthalimide $(40 \mathrm{mg}, 0.3 \mathrm{mmol}$ ) was added in one portion. The reaction mixture was subjected to microwave irradiation at $100^{\circ} \mathrm{C}(300 \mathrm{~W})$ for 10 minutes, concentrated onto silica gel, and chromatographed (0-20\% EtOAc/hexanes) to yield Phth-9-1 as colorless oil. The structure of Phth-9-1 was confirmed by conversion to the corresponding compound 
Phth-9-2 (see below). Into a round bottom flask was dissolved 2-[(2R,3S,6R)-6-allyl-2-(tert-butyldimethyl-silanyloxymethyl)-3,6-dihydro-2 $H$-pyran-3-yloxy]-isoindole-1,3-dione (Phth-9-1) (100 mg, $0.233 \mathrm{mmol})$ in tetrahydrofuran $(1 \mathrm{~mL})$ under an atmosphere of argon. To the reaction was added 500 $\mu \mathrm{l}$ of $1 \mathrm{M}$ of tetra- $n$-butylammonium fluoride in tetrahydrofuran $(500 \mu \mathrm{L})$ and acetic acid $(30 \mu \mathrm{L}, 0.50$ $\mathrm{mmol})$. The reaction was allowed to stir at room temperature. After $24 \mathrm{~h}$, the reaction was concentrated onto silica gel and purified by flash column chromatography (5-60\% EtOAc/hexanes) to afford Phth-9 as a white solid $(58.7 \mathrm{mg}, 0.186 \mathrm{mmol}, 80 \%)$. m.p. 80.5-81.5 ${ }^{\circ} \mathrm{C} .{ }^{1} \mathrm{HNMR}\left(400 \mathrm{MHz}, \mathrm{CDCl}_{3}\right) \delta 7.85-$ $7.40(\mathrm{~m}, 4 \mathrm{H}), 6.20-5.94(\mathrm{dd}, 2 \mathrm{H}, J=10.4 \mathrm{~Hz}), 5.83-5.72$ (dddd, $1 \mathrm{H}, J=7.2,3.2,3.6,2.8,7.6,4.0 \mathrm{~Hz}$ ), 4.63-4.61 (dd, $1 \mathrm{H}, J=1.6,5.2 \mathrm{~Hz}), 4.25-4.21(\mathrm{~m}, 1 \mathrm{H}), 4.05-3.94(\mathrm{~m}, 3 \mathrm{H}), 2.46-2.24(\mathrm{qt}, 2 \mathrm{H}, J=8.0$, $6.8 \mathrm{~Hz}) ;{ }^{13} \mathrm{C}\left(100 \mathrm{MHz}, \mathrm{CDCl}_{3}\right) \delta 134.8,134.2,133.8,128.9,123.8,117.7$ 79.1, 71.8, 70.6, 62.3, 37.7; IR (thin film) vmax 3312, 2966, 2933, 1785, 1730, 1642, 1467, 1375, 1310, 1267, 1186, 1121, 1079, $1049 \mathrm{~cm}^{-1}$; LRMS $[\mathrm{M}+\mathrm{H}]^{+}$calculated for $\mathrm{C}_{17} \mathrm{H}_{18} \mathrm{NO}_{5}: 316.1185$, found: $315.9 ;[\alpha]_{\mathrm{D}}^{23}=56.1^{\circ}(\mathrm{c}=$ $0.536, \mathrm{CH}_{2} \mathrm{Cl}_{2}$ ).

Phth-9-2 (2R,3S,6R)-6-Allyl-2-hydroxymethyl-3,6-dihydro-2H-pyran-3-ol: 2-((2R,3S,6R)-6-Allyl2-hydroxymethyl-3,6-dihydro-2H-pyran-3-yloxy)-isoindole-1,3-dione (Phth-9-1, $80 \mathrm{mg}, 0.2 \mathrm{mmol}$ ), $\mathrm{Mo}(\mathrm{CO})_{6}(50 \mathrm{mg}, \quad 0.2 \mathrm{mmol})$, and $\mathrm{Et}_{3} \mathrm{~N}(420 \mu \mathrm{L}, 30 \mathrm{mmol})$ were refluxed at $80^{\circ} \mathrm{C}$ in $\mathrm{MeCN} / \mathrm{H}_{2} \mathrm{O}$ $(15: 1,2 \mathrm{~mL})$ for $24 \mathrm{~h}^{15}$ The reaction was concentrated and purified by flash chromatography over silica gel (5-80\% EtOAc/hexanes) to afford a yellow oil $(23.8 \mathrm{mg}, 0.14 \mathrm{mmol}, 70 \%)$. Spectral data obtained for Phth-27-2 were in agreement with those reported by Nicolaou and coworkers. ${ }^{16}$

Acetic acid 2-acetoxymethyl-6-[2-(1,3-dioxo-1,3-dihydro-isoindol-2-yloxy)-ethyl]-3,6-dihydro-2H-

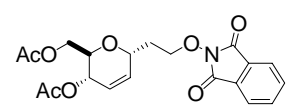

Phth-10 pyran-3-yl ester (Phth-10): To a round bottom flask was added acetic acid 3acetoxy-6-(2-hydroxy-ethyl)-3,6-dihydro-2H-pyran-2-ylmethyl ester (174.2 mg, $0.675 \mathrm{mmol}), N$-hydroxyphthalimide (143.1 mg, $0.877 \mathrm{~mol})$, triphenylphosphine $(230.0 \mathrm{mg}, 0.877 \mathrm{mmol})$ and anhydrous tetrahydrofuran $(5.00 \mathrm{~mL})$. The solution was cooled to $0{ }^{\circ} \mathrm{C}$ and diisopropyl azodicarboxylate $(172.7 \mu \mathrm{L}, 0.877 \mathrm{mmol})$ was added dropwise. The reaction mixture was stirred for $5 \mathrm{~h}\left(0{ }^{\circ} \mathrm{C}-\mathrm{rt}\right)$, concentrated and chromatographed on silica gel $(0-40 \%$ EtOAc/Hexane) to afford the desired product Phth-10 as a white solid (231 mg, $0.574 \mathrm{mmol}, 85 \%)$. m.p. 66.0-68.0 ${ }^{\circ} \mathrm{C} ;{ }^{1} \mathrm{H}$ NMR $\left(400 \mathrm{MHz}, \mathrm{CDCl}_{3}\right) \delta 7.72(\mathrm{~m}, 2 \mathrm{H}), 7.68(\mathrm{~m}, 2 \mathrm{H}), 5.94(\mathrm{~d}, 1 \mathrm{H}, J=10.4$ $\mathrm{Hz}), 5.74(\mathrm{~d}, 1 \mathrm{H}, J=10.4 \mathrm{~Hz}), 5.03(\mathrm{~m}, 1 \mathrm{H}), 4.50(\mathrm{~m}, 1 \mathrm{H}), 4.24(\mathrm{~m}, 2 \mathrm{H}), 4.17(\mathrm{dd}, 1 \mathrm{H}, J=7.6,12.4$ $\mathrm{Hz}), 4.07(\mathrm{dd}, 1 \mathrm{H}, J=1.6,11.2 \mathrm{~Hz}), 3.84(\mathrm{~m}, 1 \mathrm{H}), 1.99(\mathrm{~m}, 8 \mathrm{H}) ;{ }^{13} \mathrm{C} \mathrm{NMR}\left(100 \mathrm{MHz}, \mathrm{CDCl}_{3}\right) \delta$ $170.8,170.4,163.5,134.5,132.9,128.7,123.8,123.5,75.0,69.7,68.3,64.9,62,7,31.7,21.0,20.7$; IR (thin film) vmax 3497, 2958, 2924, 2898, 2485, 1842, 1789, 1715, 1610, 1463, 1373, 1228, 1187 , 1043, 1017, 976, 874, $701 \mathrm{~cm}^{-1}$; CIHRMS $[\mathrm{M}+\mathrm{H}]^{+}$calculated for $\mathrm{C}_{20} \mathrm{H}_{22} \mathrm{NO}_{8}$ : 404.1363, found: $404.1370 ;[\alpha]_{\mathrm{D}}^{23}=58.0^{\circ}\left(\mathrm{c}=0.455, \mathrm{CH}_{2} \mathrm{Cl}_{2}\right)$.

Phth-11 Pprepared according to: Renaudet, O.; Dumy, P. Tetrahedron Lett. 2001, 42, 7575-7578.

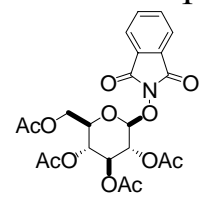

Phth-11

\footnotetext{
${ }^{15}$ Takacs, J.M.; Schroeder, S.D.; Han, J.; Gifford, M.; Jiang, X-T; Saleh, T.; Vayalakkada, S.; Yap, A.H., Org. Lett., 2003, 5, 3595-3598.

${ }^{16}$ Nicolaou, K.C.; Duggan, M.E.; Hwang, C-K, J. Am. Chem. Soc. 1989, 111, 6666-6675.
} 
2-((S)-2-Hydroxy-2-((3aR,5R,6S,6aR)-6-hydroxy-2,2-dimethyl-tetrahydrofuro[2,3-d][1,3]dioxol-

5-yl)ethoxy)isoindoline-1,3-dione (Phth-12): A $100 \mathrm{~mL}$ round-bottom flask was

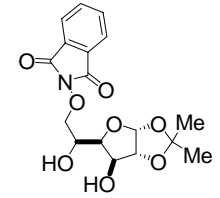

Phth-12 charged with 1,2-O-isopropylidene- $\alpha-D$-glucofuranose $(2.23 \mathrm{~g}, 10.1 \mathrm{mmol})$ and $N$ hydroxyphthalimide $(0.750 \mathrm{~g}, 4.60 \mathrm{mmol})$. The reagents were dissolved in tetrahydrofuran $(50.0 \mathrm{~mL})$ and stirred at room temperature. Triphenylphosphine $(1.20$ $\mathrm{g}, 4.60 \mathrm{mmol})$ and diisopropyl azodicarboxylate $(0.905 \mathrm{~mL}, 4.60 \mathrm{mmol})$ were added. The solution was stirred at room temperature for 8 hours. The solution was concentrated under reduced pressure. The resulting oil was hydrolyzed with water $(100 \mathrm{~mL})$. The organic layer was extracted with ethyl acetate $(3 \times 75 \mathrm{~mL})$, washed with saturated sodium chloride $(250 \mathrm{~mL})$, dried with sodium sulfate, filtered, and concentrated under reduced pressure. The crude product mixture was further purified by flash column chromatography over silica gel (hexane:EtOAc, gradient of 10:1 to 1:1) to afford the product Phth-12 as a white solid (1.21 g, 3.31 mmol, 72\%). m.p. 163.5-164.5 ${ }^{\circ} \mathrm{C} ;{ }^{1} \mathrm{H}$ NMR $\left(400 \mathrm{MHz}, \mathrm{CDCl}_{3}\right) \delta 7.81(\mathrm{~m}, 2 \mathrm{H}), 7.75(\mathrm{~m}, 2 \mathrm{H}), 5.88(\mathrm{~d}$, $1 \mathrm{H}, J=3.6 \mathrm{~Hz}), 4.50(\mathrm{~d}, 1 \mathrm{H}, J=3.6 \mathrm{~Hz}), 4.46(\mathrm{dd}, 1 \mathrm{H}, J=1.2,11.6 \mathrm{~Hz}), 4.40(\mathrm{~d}, 1 \mathrm{H}, J=2.4 \mathrm{~Hz})$, $4.30(\mathrm{~d}, 1 \mathrm{H}, J=7.6 \mathrm{~Hz}), 4.20(\mathrm{~m}, 3 \mathrm{H}), 4.04(\mathrm{dd}, 1 \mathrm{H}, J=2.4,7.6 \mathrm{~Hz}), 3.19(\mathrm{~s}, 1 \mathrm{H}), 1.44(\mathrm{~s}, 3 \mathrm{H}), 1.27$ $(\mathrm{s}, 3 \mathrm{H}) ;{ }^{13} \mathrm{C} \mathrm{NMR}\left(100 \mathrm{MHz}, \mathrm{CDCl}_{3}\right) \delta 164.4,135.0,128.7,124.0,111.9,105.2,84.9,81.3,79.7$, 75.1, 67.1, 26.9, 26.3; IR (thin film) vmax 3427, 2979, 2955, 1787, 1733, 1462, 1415, 1377, 1324, $1276,1252,1221 \mathrm{~cm}^{-1}$; CIHRMS [M] $]^{+}$calculated for $\mathrm{C}_{17} \mathrm{H}_{19} \mathrm{NO}_{8}: 365.1111$, found: $365.1129 ;[\alpha]_{\mathrm{D}}{ }^{23}=$ $+31.2^{\circ}\left(\mathrm{c}=0.573, \mathrm{CH}_{2} \mathrm{Cl}_{2}\right)$.

Scheme 12. Synthesis of 33.<smiles>O=C1c2ccccc2C(=O)N1O</smiles>

Phth-33-1

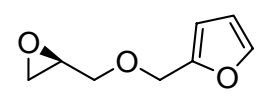

32

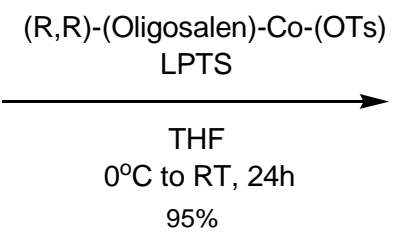

$95 \%$

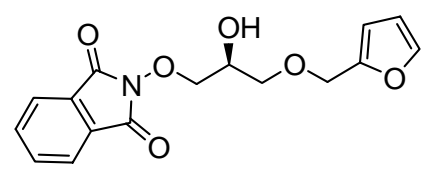

33

2-[3-(Furan-2-ylmethoxy)-2-hydroxy-propoxy]-isoindole-1,3-dione (33):A $10 \mathrm{~mL}$ round-bottom flask was charged with $N$-hydroxyphthalimide (Phth-33-1) $(0.353 \mathrm{~g}, 2.16 \mathrm{mmol})$

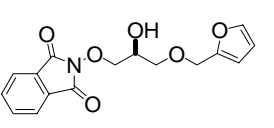

33 and (S)-2-((oxiran-2-ylmethoxy)methyl)furan (32) $(0.350 \mathrm{~g}, 2.27 \mathrm{mmol})$. The reagents were dissolved in tetrahydrofuran $(0.5 \mathrm{~mL})$ and the solution was cooled to $0{ }^{\circ} \mathrm{C}$. $(R, R)$-(Oligosalen)Co(LPTS $)^{17}(0.216 \mathrm{~g}, 0.2 \mathrm{mmol})$ and lutidinium $p$ toluene sulfonate $(30.2 \mathrm{mg}, 0.108 \mathrm{~mol})$ were added. The solution was stirred from $0{ }^{\circ} \mathrm{C}$ to room temperature over $16 \mathrm{~h}$. The reaction was diluted with $5 \mathrm{~mL}$ ethyl acetate and filtered through a plug of silica gel. The plug was washed with $20 \mathrm{~mL}$ ethyl acetate and the filtrate was concentrated under reduced pressure. The crude product was further purified by flash column chromatography over silica gel (5:1 to 1:2 hexane:EtOAc) to afford $\mathbf{3 3}$ as a pale yellow oil $(649 \mathrm{mg}$, $2.05 \mathrm{mmol}, 95 \%) .{ }^{1} \mathrm{H}$ NMR $\left(400 \mathrm{MHz}, \mathrm{CDCl}_{3}\right) \delta 7.85(\mathrm{~m}, 2 \mathrm{H}), 7.78(\mathrm{~m}, 2 \mathrm{H}), 7.38(\mathrm{~s}, 1 \mathrm{H}), 6.32(\mathrm{~s}$, 2H), $4.49(\mathrm{~s}, 2 \mathrm{H}), 4.32(\mathrm{~m}, 1 \mathrm{H}), 4.16(\mathrm{~m}, 1 \mathrm{H}), 4.07(\mathrm{~m}, 1 \mathrm{H}), 3.65(\mathrm{~m}, 1 \mathrm{H}), 3.59(\mathrm{~m}, 2 \mathrm{H}) ;{ }^{13} \mathrm{C} \mathrm{NMR}$ $\left(100 \mathrm{MHz}, \mathrm{CDCl}_{3}\right) \delta 170.7,163.2,151.3,142.6,134.5,128.7,123.4,110.0,109.3,80.1,70.0,67.6$, 65.0; IR (thin film) vmax 3501, 3147, 3120, 2908, 1788, 1722, 1612, 1503, 1468, $1375 \mathrm{~cm}^{-1}$; CIHRMS $[\mathrm{M}+\mathrm{H}]^{+}$calculated for $\mathrm{C}_{16} \mathrm{H}_{15} \mathrm{NO}_{6}: 318.0996$, found: $318.0980 ;[\alpha]_{\mathrm{D}}^{23}=+27.1^{\circ}\left(\mathrm{c}=0.945, \mathrm{CH}_{2} \mathrm{Cl}_{2}\right)$.

${ }^{17}$ Ready, J. M.; Jacobsen, E. N. J. Am. Chem. Soc. 2001, 123, 2687-2688. 


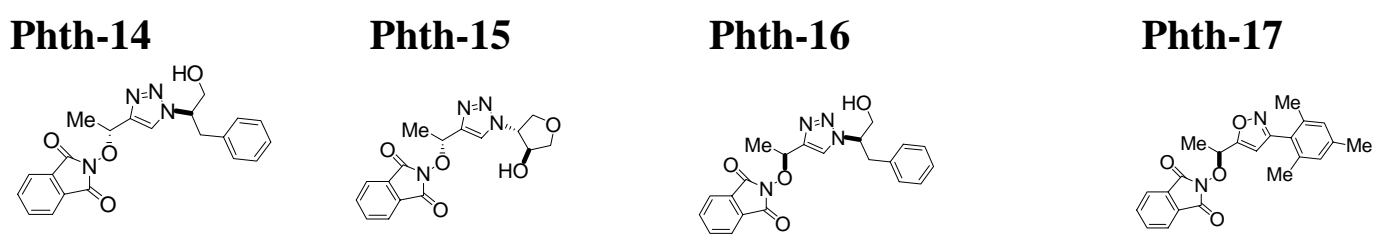

Prepared according to: Su, S.; Giguere, J. R.; Schaus, S. E.; Porco, J. A. Jr., Tetrahedron 2004, 39, 8645-8657.

\subsection{Representative procedure for phthalimide deprotection. ${ }^{18}$}

2-(((2R,3S,6S)-3-(4-Methoxyphenoxy)-3,6-dihydro-6-phenyl-2H-pyran-2-yl)methoxy) isoindoline1,3-dione (7): Phth-7 (215 mg, $0.473 \mathrm{mmol}$ ) was dissolved in $\mathrm{CH}_{2} \mathrm{Cl}_{2}$ and transferred

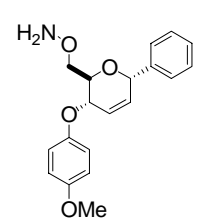
to a $5 \mathrm{~mL}$ reaction vessel on the Quest 210 synthesizer containing pre-swelled PSdiethylenetriamine $(473 \mathrm{mg}, 3.0 \mathrm{mmol} / \mathrm{g}, 1.42 \mathrm{mmol})$. The reaction was agitated for $12 \mathrm{~h}$. After filtration, the resin was washed with an additional $\mathrm{CH}_{2} \mathrm{Cl}_{2}(3 \mathrm{~mL} \times 3)$ and the combined filtrate was concentrated to afford the desired alkoxyamine as a colorless oil ( $147 \mathrm{mg}, 0.45 \mathrm{mmol}, 95 \%)$.

Other alkoxyamine monomers were prepared using a similar procedure. For substrates with limited solubility in $\mathrm{CH}_{2} \mathrm{Cl}_{2}$, an appropriate amount of $\mathrm{MeOH}$ (10-30\%) was added.

\footnotetext{
${ }^{18}$ Stangier, P.; Hindsgaul, O. Synlett 1996, 2, 179.
} 
Crystals of compound 22-5 suitable for x-ray analysis were obtained by slow evaporation from hexane. Crystallographic data have been deposited with the Cambridge Crystallographic Data Centre (CCDC \# 260139). Copies of the data can be obtained free of charge on application to the CCDC, 12 Union Road, Cambridge CB21EZ, UK (fax: (+44)-1223-336-033; e-mail: deposit@,ccdc.cam.ac.uk.

Table 1. Crystal data and structure refinement for 22-5.

Identification code

Empirical formula

Formula weight

Temperature

Wavelength

Crystal system

Space group

Unit cell dimensions

Volume

$\mathrm{Z}$

Density (calculated)

Absorption coefficient

$\mathrm{F}(000)$

Crystal size

Theta range for data collection

Index ranges

Reflections collected

Independent reflections

Completeness to theta $=30.51^{\circ}$

Absorption correction

Max. and min. transmission

Refinement method

Data / restraints / parameters

Goodness-of-fit on $\mathrm{F}^{2}$

Final R indices [I $>2 \operatorname{sigma}(\mathrm{I})]$

$\mathrm{R}$ indices (all data)

Absolute structure parameter

Largest diff. peak and hole
22-5

$\mathrm{C} 23 \mathrm{H} 23 \mathrm{Br} \mathrm{O} 5$

459.32

173(2) K

$0.71073 \AA$

Monoclinic

P2(1)

$\mathrm{a}=10.0462(7) \AA \quad \alpha=90^{\circ}$.

$\mathrm{b}=5.3870(4) \AA \quad \beta=100.504(2)^{\circ}$.

$\mathrm{c}=19.6191(14) \AA \quad \gamma=90^{\circ}$.

1043.97(13) $\AA^{3}$

2

$1.461 \mathrm{Mg} / \mathrm{m}^{3}$

$2.000 \mathrm{~mm}^{-1}$

472

$1.00 \times 0.30 \times 0.15 \mathrm{~mm}^{3}$

2.48 to $30.51^{\circ}$.

$-14<=\mathrm{h}<=14,-6<=\mathrm{k}<=7,-24<=1<=28$

8041

$5322[\mathrm{R}(\mathrm{int})=0.0252]$

$97.8 \%$

\section{SADABS}

0.7535 and 0.2396

Full-matrix least-squares on $\mathrm{F}^{2}$

$5322 / 1 / 343$

0.964

$\mathrm{R} 1=0.0384, \mathrm{wR} 2=0.0819$

$\mathrm{R} 1=0.0539, \mathrm{wR} 2=0.0869$

$0.055(10)$

0.567 and -0.503 e. $\AA^{-3}$ 
Table 2. Atomic coordinates $\left(\mathrm{x} 10^{4}\right)$ and equivalent isotropic displacement parameters $\left(\AA^{2} \times 10^{3}\right)$ for 22-5. $U(e q)$ is defined as one third of the trace of the orthogonalized $U^{i j}$ tensor.

\begin{tabular}{|c|c|c|c|c|}
\hline & $\mathrm{x}$ & $\mathrm{y}$ & $\mathrm{z}$ & $\mathrm{U}(\mathrm{eq})$ \\
\hline $\operatorname{Br}(1)$ & $944(1)$ & $2677(1)$ & $4190(1)$ & $41(1)$ \\
\hline $\mathrm{O}(1)$ & $-5019(2)$ & $14447(4)$ & 2779(1) & $27(1)$ \\
\hline $\mathrm{O}(2)$ & $-8105(2)$ & $11847(3)$ & 1833(1) & $30(1)$ \\
\hline $\mathrm{O}(3)$ & $-12856(2)$ & $12867(6)$ & $-91(1)$ & $36(1)$ \\
\hline $\mathrm{O}(4)$ & $-4033(2)$ & 9901(4) & $2407(1)$ & $30(1)$ \\
\hline $\mathrm{O}(5)$ & $-3783(2)$ & $7402(5)$ & $1524(1)$ & $33(1)$ \\
\hline $\mathrm{C}(1)$ & $-5547(3)$ & $15648(6)$ & $3321(1)$ & $29(1)$ \\
\hline$C(2)$ & $-6994(3)$ & $16409(6)$ & $3081(2)$ & $31(1)$ \\
\hline$C(3)$ & $-7704(3)$ & $15654(5)$ & 2481(2) & $29(1)$ \\
\hline$C(4)$ & $-7166(3)$ & $13852(5)$ & 2019(1) & $26(1)$ \\
\hline$C(5)$ & $-5894(2)$ & $12602(7)$ & $2422(1)$ & $25(1)$ \\
\hline$C(6)$ & $-5117(3)$ & 11212(6) & $1960(2)$ & $30(1)$ \\
\hline$C(7)$ & $-5341(3)$ & $14054(7)$ & $3983(2)$ & $34(1)$ \\
\hline $\mathrm{C}(8)$ & $-3884(3)$ & $13459(10)$ & $4251(2)$ & $64(2)$ \\
\hline$C(9)$ & $-3230(5)$ & $13519(10)$ & $4787(3)$ & $89(2)$ \\
\hline$C(10)$ & $-9526(3)$ & $14298(5)$ & $912(1)$ & $26(1)$ \\
\hline $\mathrm{C}(11)$ & $-10738(3)$ & $14430(6)$ & $440(1)$ & $27(1)$ \\
\hline$C(12)$ & $-11707(2)$ & $12573(8)$ & $407(1)$ & $25(1)$ \\
\hline$C(13)$ & $-11482(3)$ & $10585(5)$ & $859(1)$ & $25(1)$ \\
\hline$C(14)$ & $-10262(3)$ & $10435(5)$ & 1333(1) & $24(1)$ \\
\hline$C(15)$ & $-9288(2)$ & $12262(5)$ & $1353(1)$ & $23(1)$ \\
\hline$C(16)$ & $-13857(3)$ & $10966(8)$ & $-119(2)$ & $41(1)$ \\
\hline$C(17)$ & $-3451(2)$ & $8036(7)$ & $2117(1)$ & $27(1)$ \\
\hline $\mathrm{C}(18)$ & $-2340(2)$ & $6859(5)$ & 2619(1) & $24(1)$ \\
\hline$C(19)$ & $-1939(2)$ & 7774(9) & $3294(1)$ & $31(1)$ \\
\hline$C(20)$ & $-944(3)$ & $6523(6)$ & $3761(1)$ & $32(1)$ \\
\hline$C(21)$ & $-371(2)$ & $4424(6)$ & $3545(1)$ & $28(1)$ \\
\hline $\mathrm{C}(22)$ & $-721(3)$ & $3513(6)$ & $2878(2)$ & $31(1)$ \\
\hline $\mathrm{C}(23)$ & $-1715(3)$ & $4776(6)$ & 2413(1) & $29(1)$ \\
\hline
\end{tabular}


Crystals of compound 28-4 suitable for x-ray analysis were obtained by slow evaporation from $\mathrm{CH}_{2} \mathrm{Cl}_{2}$. Crystallographic data have been deposited with the Cambridge Crystallographic Data Centre (CCDC \# 260141). Copies of the data can be obtained free of charge on application to the CCDC, 12 Union Road, Cambridge CB21EZ, UK (fax: (+44)-1223-336-033; e-mail: deposit@ccdc.cam.ac.uk.

Table 3. Crystal data and structure refinement for 28-4.

\begin{tabular}{|c|c|c|}
\hline Identification code & \multicolumn{2}{|l|}{ 28-4 } \\
\hline Empirical formula & \multicolumn{2}{|c|}{ C14 H15 N O5 * C H2 Cl2 } \\
\hline Formula weight & \multicolumn{2}{|l|}{362.20} \\
\hline Temperature & \multicolumn{2}{|l|}{$173(2) \mathrm{K}$} \\
\hline Wavelength & \multicolumn{2}{|l|}{$0.71073 \AA$} \\
\hline Crystal system & \multicolumn{2}{|l|}{ Monoclinic } \\
\hline Space group & \multicolumn{2}{|l|}{$\mathrm{P} 2(1) / \mathrm{c}$} \\
\hline \multirow[t]{3}{*}{ Unit cell dimensions } & $\mathrm{a}=13.2845(6) \AA$ & $\alpha=90^{\circ}$. \\
\hline & $\mathrm{b}=7.8086(4) \AA$ & $\beta=101.8200(10)^{\circ}$. \\
\hline & $\mathrm{c}=15.4619(7) \AA$ & $\gamma=90^{\circ}$ \\
\hline Volume & \multicolumn{2}{|l|}{$1569.90(13) \AA^{3}$} \\
\hline Z & \multicolumn{2}{|l|}{4} \\
\hline Density (calculated) & \multicolumn{2}{|l|}{$1.532 \mathrm{Mg} / \mathrm{m}^{3}$} \\
\hline Absorption coefficient & \multicolumn{2}{|l|}{$0.439 \mathrm{~mm}^{-1}$} \\
\hline $\mathrm{F}(000)$ & \multicolumn{2}{|l|}{752} \\
\hline Crystal size & \multicolumn{2}{|c|}{$0.50 \times 0.30 \times 0.15 \mathrm{~mm}^{3}$} \\
\hline Theta range for data collection & \multicolumn{2}{|l|}{2.69 to $28.27^{\circ}$. } \\
\hline Index ranges & \multicolumn{2}{|c|}{$-17<=\mathrm{h}<=14,-10<=\mathrm{k}<=10,-20<=\mathrm{l}<=20$} \\
\hline Reflections collected & \multicolumn{2}{|l|}{10965} \\
\hline Independent reflections & \multicolumn{2}{|c|}{$3773[\mathrm{R}(\mathrm{int})=0.0270]$} \\
\hline Completeness to theta $=28.27^{\circ}$ & \multicolumn{2}{|l|}{$96.6 \%$} \\
\hline Absorption correction & \multicolumn{2}{|c|}{ Semiempirical by SADABS } \\
\hline Max. and min. transmission & \multicolumn{2}{|l|}{0.9371 and 0.8105} \\
\hline Refinement method & \multicolumn{2}{|c|}{ Full-matrix least-squares on $\mathrm{F}^{2}$} \\
\hline Data / restraints / parameters & \multicolumn{2}{|l|}{$3773 / 0 / 276$} \\
\hline Goodness-of-fit on $\mathrm{F}^{2}$ & \multicolumn{2}{|l|}{1.275} \\
\hline Final $R$ indices $[\mathrm{I}>2 \operatorname{sigma}(\mathrm{I})]$ & \multicolumn{2}{|c|}{$\mathrm{R} 1=0.0538, \mathrm{wR} 2=0.1591$} \\
\hline $\mathrm{R}$ indices (all data) & \multicolumn{2}{|c|}{$\mathrm{R} 1=0.0646, \mathrm{wR} 2=0.1665$} \\
\hline Largest diff. peak and hole & \multicolumn{2}{|c|}{1.068 and -0.683 e. $\AA^{-3}$} \\
\hline
\end{tabular}


Table 4. Atomic coordinates $\left(\mathrm{x} 10^{4}\right)$ and equivalent isotropic displacement parameters $\left(\AA^{2} \times 10^{3}\right)$ for 28-4. $U(e q)$ is defined as one third of the trace of the orthogonalized $U^{i j}$ tensor.

\begin{tabular}{|c|c|c|c|c|}
\hline & $\mathrm{x}$ & $\mathrm{y}$ & $\mathrm{z}$ & $\mathrm{U}(\mathrm{eq})$ \\
\hline $\mathrm{O}(1)$ & $817(1)$ & 7871(2) & $9692(1)$ & $23(1)$ \\
\hline $\mathrm{O}(2)$ & $574(1)$ & $9655(2)$ & 7781(1) & $25(1)$ \\
\hline $\mathrm{O}(3)$ & $-1688(1)$ & $8161(2)$ & $6415(1)$ & $30(1)$ \\
\hline $\mathrm{O}(4)$ & $-4202(1)$ & $8437(2)$ & 9444(1) & $27(1)$ \\
\hline $\mathrm{O}(5)$ & $-1435(1)$ & $5073(2)$ & $9357(1)$ & $26(1)$ \\
\hline $\mathrm{N}(1)$ & $-2951(1)$ & 6443(2) & $9377(1)$ & $18(1)$ \\
\hline $\mathrm{C}(1)$ & $118(2)$ & $7566(2)$ & $8872(1)$ & $15(1)$ \\
\hline$C(2)$ & $638(2)$ & 7927(2) & $8117(2)$ & $20(1)$ \\
\hline$C(3)$ & $25(2)$ & $8298(3)$ & 7234(1) & $22(1)$ \\
\hline$C(4)$ & $-1121(2)$ & $8408(2)$ & $7130(1)$ & $20(1)$ \\
\hline$C(5)$ & $-1491(2)$ & 8923(2) & $7930(1)$ & $15(1)$ \\
\hline$C(6)$ & $-2377(2)$ & $9766(2)$ & $7857(1)$ & $18(1)$ \\
\hline$C(7)$ & $-2674(2)$ & $10542(3)$ & $8656(1)$ & $20(1)$ \\
\hline$C(8)$ & $-2435(2)$ & 9342(3) & $9467(1)$ & $17(1)$ \\
\hline $\mathrm{C}(9)$ & $-1478(2)$ & $8207(2)$ & $9512(1)$ & $15(1)$ \\
\hline$C(10)$ & $-816(1)$ & $8727(2)$ & $8844(1)$ & $13(1)$ \\
\hline$C(11)$ & $-3317(2)$ & 8094(3) & $9434(1)$ & $18(1)$ \\
\hline$C(12)$ & $-1912(2)$ & 6393(2) & 9399(1) & $17(1)$ \\
\hline$C(13)$ & $-3607(2)$ & 4927(3) & $9327(1)$ & $23(1)$ \\
\hline$C(14)$ & $-3590(2)$ & 4154(4) & $10230(2)$ & $36(1)$ \\
\hline $\mathrm{Cl}(1)$ & $3255(1)$ & $-924(1)$ & $7908(1)$ & $59(1)$ \\
\hline $\mathrm{Cl}(2)$ & $4540(1)$ & 2079(1) & 7813(1) & $40(1)$ \\
\hline $\mathrm{C}(1 \mathrm{~S})$ & $3848(2)$ & $879(4)$ & $8471(2)$ & $36(1)$ \\
\hline
\end{tabular}


Crystals of compound 29 suitable for x-ray analysis were obtained by slow evaporation from $\mathrm{CH}_{2} \mathrm{Cl}_{2}$. Crystallographic data have been deposited with the Cambridge Crystallographic Data Centre (CCDC \# 260140). Copies of the data can be obtained free of charge on application to the CCDC, 12 Union Road, Cambridge CB21EZ, UK (fax: (+44)-1223-336-033; e-mail: deposit@,ccdc.cam.ac.uk.

Table 5. Crystal data and structure refinement for 29.

Identification code

Empirical formula

Formula weight

Temperature

Wavelength

Crystal system

Space group

Unit cell dimensions

Volume

Z

Density (calculated)

Absorption coefficient

$\mathrm{F}(000)$

Crystal size

Theta range for data collection

Index ranges

Reflections collected

Independent reflections

Completeness to theta $=28.28^{\circ}$

Absorption correction

Max. and min. transmission

Refinement method

Data / restraints / parameters

Goodness-of-fit on $\mathrm{F}^{2}$

Final R indices [I $>2 \operatorname{sigma}(\mathrm{I})]$

$\mathrm{R}$ indices (all data)

Absolute structure parameter

Largest diff. peak and hole
29

C16 H15 N3 O5

329.31

173(2) K

$0.71073 \AA$

Orthorhombic

P2(1)2(1)2(1)

$\mathrm{a}=7.2933(10) \AA \quad \alpha=90^{\circ}$.

$\mathrm{b}=8.2345(12) \AA \quad \beta=90^{\circ}$.

$\mathrm{c}=24.418(3) \AA \quad \gamma=90^{\circ}$.

$1466.4(3) \AA^{3}$

4

$1.492 \mathrm{Mg} / \mathrm{m}^{3}$

$0.113 \mathrm{~mm}^{-1}$

688

$0.50 \times 0.15 \times 0.10 \mathrm{~mm}^{3}$

2.61 to $28.28^{\circ}$.

$-9<=\mathrm{h}<=8,-10<=\mathrm{k}<=7,-21<=\mathrm{l}<=32$

7736

3575 [R(int) $=0.0457]$

$99.7 \%$

Semiempirical by SADABS

0.9888 and 0.9456

Full-matrix least-squares on $\mathrm{F}^{2}$

3575 / 0 / 277

1.027

$\mathrm{R} 1=0.0519, \mathrm{wR} 2=0.0941$

$\mathrm{R} 1=0.0850, \mathrm{wR} 2=0.1045$

$0.4(13)$

0.205 and -0.264 e. $\AA^{-3}$ 
Table 6. Atomic coordinates $\left(\mathrm{x} 10^{4}\right)$ and equivalent isotropic displacement parameters $\left(\AA^{2} \times 10^{3}\right)$ for 29. $U(\mathrm{eq})$ is defined as one third of the trace of the orthogonalized Uij tensor.

\begin{tabular}{lcccc}
\hline & $\mathrm{x}$ & $\mathrm{y}$ & $\mathrm{z}$ & $\mathrm{U}(\mathrm{eq})$ \\
\hline $\mathrm{O}(1)$ & & & & \\
$\mathrm{O}(2)$ & $5897(2)$ & $8344(2)$ & $11265(1)$ & $38(1)$ \\
$\mathrm{O}(3)$ & $4917(3)$ & $8545(3)$ & $12514(1)$ & $49(1)$ \\
$\mathrm{O}(4)$ & $304(3)$ & $8228(3)$ & $12769(1)$ & $46(1)$ \\
$\mathrm{O}(5)$ & $951(2)$ & $6254(2)$ & $9878(1)$ & $31(1)$ \\
$\mathrm{N}(1)$ & $5606(2)$ & $4485(2)$ & $11012(1)$ & $33(1)$ \\
$\mathrm{N}(2)$ & $2949(3)$ & $5972(2)$ & $11144(1)$ & $24(1)$ \\
$\mathrm{N}(3)$ & $1638(3)$ & $6672(2)$ & $10788(1)$ & $24(1)$ \\
$\mathrm{C}(1)$ & $3594(3)$ & $5213(2)$ & $10302(1)$ & $22(1)$ \\
$\mathrm{C}(2)$ & $4162(3)$ & $8548(3)$ & $11519(1)$ & $26(1)$ \\
$\mathrm{C}(3)$ & $4419(4)$ & $9487(4)$ & $12043(1)$ & $40(1)$ \\
$\mathrm{C}(4)$ & $3124(4)$ & $9311(4)$ & $12494(1)$ & $45(1)$ \\
$\mathrm{C}(5)$ & $1552(4)$ & $8177(3)$ & $12442(1)$ & $32(1)$ \\
$\mathrm{C}(6)$ & $1472(3)$ & $7062(3)$ & $11945(1)$ & $25(1)$ \\
$\mathrm{C}(7)$ & $3312(3)$ & $6903(3)$ & $11647(1)$ & $24(1)$ \\
$\mathrm{C}(8)$ & $-16(4)$ & $7778(3)$ & $11563(1)$ & $26(1)$ \\
$\mathrm{C}(9)$ & $6379(4)$ & $4474(3)$ & $9812(1)$ & $27(1)$ \\
$\mathrm{C}(10)$ & & & $24(1)$ \\
$\mathrm{C}(11)$ & & & $26(1)$ \\
$\mathrm{C}(12)$ & $192(3)$ & $6833(3)$ & $11032(1)$ & $23(1)$ \\
$\mathrm{C}(13)$ & $1942(3)$ & $6067(3)$ & $10275(1)$ & $30(1)$ \\
$\mathrm{C}(14)$ & $4247(3)$ & $5160(3)$ & $10845(1)$ & $30(1)$ \\
$\mathrm{C}(15)$ & $4483(3)$ & $4424(3)$ & $9851(1)$ & $28(1)$ \\
$\mathrm{C}(16)$ & $3437(4)$ & $3592(3)$ & $9467(1)$ & $9031(1)$ \\
\hline
\end{tabular}




\section{Analytical Scale Rehearsal for Library Syntheses}

General procedure for analytical scale rehearsal: $0.0033 \mathrm{mmol}$ of alkoxyamine (1.1 equiv.) in $1 \mathrm{~mL}$ EtOAc was transferred to a $5 \mathrm{~mL}$ microwave reaction vessel followed by addition of $0.0030 \mathrm{mmol}$ of ketone in $1 \mathrm{~mL}$ EtOAc. The solvents were removed in vacuo using the GeneVac ${ }^{\circledR}$ EZ-2 evaporator. After evaporation, $\mathrm{AcOH}(0.0006 \mathrm{mmol}, 20 \%)$ or PPTS $(0.0006 \mathrm{mmol}, 20 \%)$ was added followed by addition of $0.5 \mathrm{~mL}$ of THF. The reaction mixture was carried out following the conditions listed in Table 7 and concentrated using the GeneVac ${ }^{\circledR}$ EZ-2 evaporation system. The crude product was dissolved in $1 \mathrm{~mL}$ of $\mathrm{CH}_{2} \mathrm{Cl}_{2}$, and transferred to a $5 \mathrm{~mL}$ reaction vessel on the Quest 210 synthesizer containing PL-MIA resin $(5.0 \mathrm{mg}, 2.57 \mathrm{mmol} / \mathrm{g}, 0.0129 \mathrm{mmol})$. Reactions employing PPTS were first dissolved in $\mathrm{CH}_{2} \mathrm{Cl}_{2}$, applied to an SLE cartridge $(1.0 \mathrm{~mL})$ containing sat. $\mathrm{NaHCO}_{3}$, eluted with $10 \mathrm{~mL}$ $\mathrm{CH}_{2} \mathrm{Cl}_{2}$, concentrated, re-dissolved in $\mathrm{CH}_{2} \mathrm{Cl}_{2}$, and subjected to scavenging as described above. The scavenging reaction was agitated for $4 \mathrm{~h}$, the filtrate was collected, and the resin was washed by additional $\mathrm{CH}_{2} \mathrm{Cl}_{2}(2 \times 5 \mathrm{~mL})$. The combined filtrate were concentrated using the GeneVac ${ }^{\circledR} \mathrm{EZ}-2$ to afford the crude product which was dissolved in MeCN and subjected to HPLC-ELSD analysis using a Waters HPLC-MS system (XTerra ${ }^{\circledR R P} 8,5 \mu \mathrm{m}, 4.6 \times 30 \mathrm{~mm}$ column) to determine product purity. Detailed reaction conditions and results are listed in Table 7.

Table 7: Oxime product purities from analytical scale rehearsal. ${ }^{a, b}$

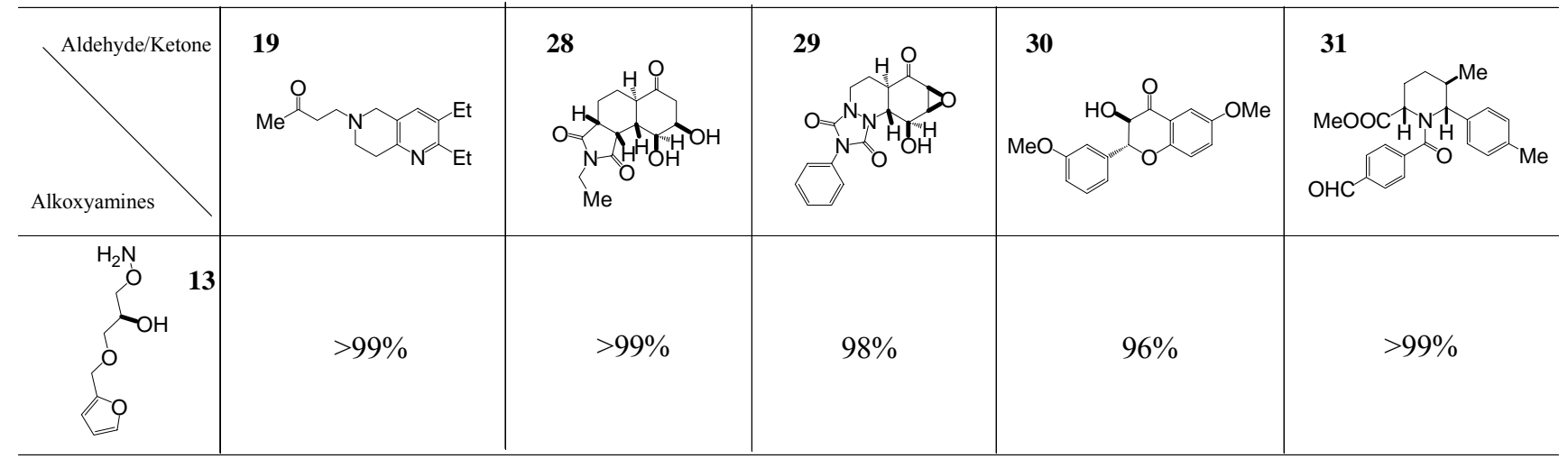

a. Product purity as determined by HPLC-ELSD analysis

b. Reaction conditions: (1) 13+29: 1.0 equiv. alkoxyamine, EtOAc, AcOH, rt, 12h; (2) 13+28, 31: 1.1 equiv. alkoxyamine, EtOAc, $\mathrm{AcOH}, \mathrm{rt}, 12 \mathrm{~h}$; (3) 13+19: 1.1 equiv. alkoxyamine, $\mathrm{AcOH}, \mathrm{THF}, 100{ }^{\circ} \mathrm{C}$, microwave $300 \mathrm{~W}, 20 \mathrm{~min}$; (4) 13+30: 1.5 equiv. alkoxyamine, PPTS, THF, $110^{\circ} \mathrm{C}$, microwave $300 \mathrm{~W}, 45 \mathrm{~min}$. 


\section{Library Synthesis}

4.1 Representative procedure for condensation of alkoxyamines and aldehyde/ketones 28, 29: Aldehyde 26 (3.8 mg, $0.0189 \mathrm{mmol}$, in $1.0 \mathrm{ml}$ EtOAc) was transferred to reaction vessel using the MiniMapper ${ }^{\mathrm{TM}}$ followed by the addition of alkoxyamine $10(5.7 \mathrm{mg}, 0.0208 \mathrm{mmol}$, in $1.0 \mathrm{ml}$ of EtOAc, 1.1 equiv.). The reaction mixture was evaporated on the GeneVac ${ }^{\mathbb{B}}$ EZ-2 evaporator. Upon completion of the evaporation, AcOH $(0.0038 \mathrm{mmol}$ in $0.2 \mathrm{ml} \mathrm{EtOAc}, 20 \%)$ was added followed by the addition of $1 \mathrm{~mL}$ EtOAc. The reaction mixture was stirred at $\mathrm{rt}$ for $3 \mathrm{~h}$, concentrated using the GeneVac ${ }^{\circledR} \mathrm{EZ}-2$, dissolved in $\mathrm{CH}_{2} \mathrm{Cl}_{2}$, and transferred to a $5 \mathrm{~mL}$ reaction vessel on the Quest 210 synthesizer containing PL-MIA resin $(20 \mathrm{mg}, 2.57 \mathrm{mmol} / \mathrm{g}, 0.051 \mathrm{mmol})$. The scavenging reaction was agitated for $4 \mathrm{~h}$, the filtrate was collected, and the resin was washed with additional $\mathrm{CH}_{2} \mathrm{Cl}_{2}(5 \mathrm{~mL} \times 2)$. The combined solution was concentrated on the GeneVac ${ }^{\circledR}$ EZ-2 to afford the desired product $(8.2 \mathrm{mg}, 0.018 \mathrm{mmol}$, $95 \%$ ). The product was dissolved in MeCN and subjected to HPLC-ELSD analysis using a Waters HPLC-MS system (XTerra ${ }^{\circledR R P} P_{8}, 5 \mu \mathrm{m}, 4.6 \times 30 \mathrm{~mm}$ column) to determine product purity. The ratio of $\mathrm{E} / \mathrm{Z}$ oxime isomers was determined by ${ }^{1} \mathrm{H}$ NMR. ${ }^{19}$

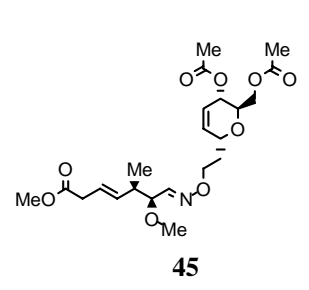

Oxime 45: HPLC-ELSD purity 95\%; E/Z = 3.7:1 ( ${ }^{1} \mathrm{H}$ NMR (400 $\left.\mathrm{MHz}, \mathrm{CDCl}_{3}\right)$ ); ${ }^{1} \mathrm{H}$ NMR (400 MHz, $\mathrm{CDCl}_{3}$, major isomer reported) $\delta 7.16(\mathrm{~d}, 1 \mathrm{H}, J=7.6 \mathrm{~Hz})$, 5.91-5.87 (m, 2H), 5.59-5.47 (m, 2H), 5.13-5.12 (m, 1H), 4.36-4.33 (m, 1H), 4.28$4.10(\mathrm{~m}, 4 \mathrm{H}), 3.91-3.87(\mathrm{~m}, 1 \mathrm{H}), 3.66(\mathrm{~s}, 3 \mathrm{H}), 3.50(\mathrm{dd}, 1 \mathrm{H}, J=6.4,8.0 \mathrm{~Hz}), 3.28$ $(\mathrm{s}, 3 \mathrm{H}), 3.04(\mathrm{~d}, 2 \mathrm{H}, J=5.6 \mathrm{~Hz}), 2.50-2.46(\mathrm{~m}, 1 \mathrm{H}), 2.07(\mathrm{~s}, 3 \mathrm{H}), 2.06(\mathrm{~s}, 3 \mathrm{H})$, $1.97-1.86(\mathrm{~m}, 2 \mathrm{H}), 1.04(\mathrm{~d}, 3 \mathrm{H}, J=6.8 \mathrm{~Hz}) ;{ }^{13} \mathrm{C} \mathrm{NMR}\left(100 \mathrm{MHz}, \mathrm{CDCl}_{3}\right.$, major isomer reported) $\delta 172.4,171.1,149.7,134.9,133.3,123.9,123.2,82.1,70.5,69.7,69.2,65.2,63.1$, 57.2, 52.0, 40.3, 38.1, 32.6, 29.9, 21.3, 21.0, 15.8; CIHRMS $[\mathrm{M}+\mathrm{H}]^{+}$calculated for $\mathrm{C}_{22} \mathrm{H}_{34} \mathrm{NO}_{9}$ : 456.2233, found: 456.2265 .

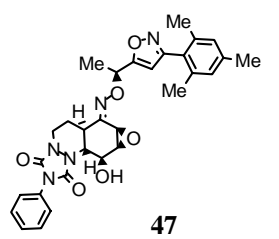

Oxime 47: HPLC-ELSD purity 95.4\%; $\mathrm{E} / \mathrm{Z}=3.3: 1\left({ }^{1} \mathrm{H} \mathrm{NMR}\left(400 \mathrm{MHz}, \mathrm{CDCl}_{3}\right)\right.$ ); ${ }^{1} \mathrm{H}$ NMR (400 MHz, $\mathrm{CDCl}_{3}$, major isomer reported) $\delta 7.52-7.45(\mathrm{~m}, 4 \mathrm{H}), 7.39-7.36$ $(\mathrm{m}, 1 \mathrm{H}), 6.94(\mathrm{~s}, 2 \mathrm{H}), 6.14(\mathrm{~s}, 1 \mathrm{H}), 5.50(\mathrm{q}, 1 \mathrm{H}, J=6.8 \mathrm{~Hz}), 4.59-4.53(\mathrm{~m}, 2 \mathrm{H}), 4.36$ $(\mathrm{d}, 1 \mathrm{H}, J=4.4 \mathrm{~Hz}), 4.15-4.11(\mathrm{~m}, 1 \mathrm{H}), 3.68(\mathrm{~d}, 1 \mathrm{H}, J=3.6 \mathrm{~Hz}), 3.23-3.17(\mathrm{~m}, 1 \mathrm{H})$, 2.94-2.89 (m, 1H), $2.33(\mathrm{~s}, 3 \mathrm{H}), 2.13(\mathrm{~s}, 6 \mathrm{H}), 1.91-1.85(\mathrm{~m}, 3 \mathrm{H}), 1.76(\mathrm{~d}, 3 \mathrm{H}, J=6.8$ $\mathrm{Hz}) ;{ }^{13} \mathrm{C} \mathrm{NMR}\left(100 \mathrm{MHz}, \mathrm{CDCl}_{3}\right.$, both isomer reported) $\delta 172.0,171.4,162.2$, 153.6, 153.2, 152.8, 139.2, 137.4, 137.2, 131.2, 129.4, 128.6, 128.5, 126.0, 125.9, 103.6, 103.0, 94.7, 74.4, 73.2, 66.4, 56.9, 53.7, 53.1, 52.5, 46.3, 44.0, 37.6, 27.1, 22.0, 21.3, 20.5, 19.0, 18.5; CIHRMS $[\mathrm{M}+\mathrm{H}]^{+}$calculated for $\mathrm{C}_{30} \mathrm{H}_{32} \mathrm{~N}_{5} \mathrm{O}_{6}: 558.2353$, found: 558.2411 .

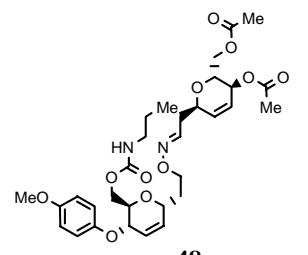

48

Oxime 48: HPLC-ELSD purity $>99 \%$; $\mathrm{E} / \mathrm{Z}=1: 1\left({ }^{1} \mathrm{H}\right.$ NMR $\left.\left(400 \mathrm{MHz}, \mathrm{CDCl}_{3}\right)\right)$; ${ }^{1} \mathrm{H}$ NMR (400 MHz, $\mathrm{CDCl}_{3}$, both isomers reported) $\delta 7.47(\mathrm{t}, 0.5 \mathrm{H}, J=6.0 \mathrm{~Hz}$ ), 6.87-6.81(m, 5.5H), 5.07-5.85 (m, 4H), 5.14-5.13 (m, 1H), $4.83(\mathrm{bs}, 1 \mathrm{H}), 4.56(\mathrm{~d}$, $1 \mathrm{H}, J=7.2 \mathrm{~Hz}), 4.44-4.41(\mathrm{~m}, 2 \mathrm{H}), 4.36-4.10(\mathrm{~m}, 5 \mathrm{H}), 4.00-3.94(\mathrm{~m}, 2 \mathrm{H}), 3.77$ (s, $3 \mathrm{H}), 3.15(\mathrm{dd}, 2 \mathrm{H}, J=6.8,13.2 \mathrm{~Hz}), 2.74-2.44(\mathrm{~m}, 2 \mathrm{H}), 2.103(\mathrm{~s}, 3 \mathrm{H}), 2.099$ (s, $3 \mathrm{H}), 2.07-1.90(\mathrm{~m}, 2 \mathrm{H}), 1.55(\mathrm{dt}, 2 \mathrm{H}, J=7.2,14.8 \mathrm{~Hz}), 0.92(\mathrm{t}, 3 \mathrm{H}, J=7.2 \mathrm{~Hz})$;

${ }^{13} \mathrm{C} \mathrm{NMR}\left(100 \mathrm{MHz}, \mathrm{CDCl}_{3}\right.$, both isomers reported) $\delta 171.1,170.7,156.4,154.5,151.3,147.7,147.4$, $132.6,132.4,132.2,124.9,124.6,124.5,117.4,114.9,70.7,70.4,70.3,70.0,69.8,68.9,64.8,64.1$,

\footnotetext{
${ }^{19}$ For determination of oxime $Z-E$ ratio by ${ }^{1} \mathrm{H}$ NMR, see: (a) Deubner, R.; Holzgrabe, U. Magn. Reson. Chem. 2002, 40, 762-766; (b) Bromidge, S. M.; Brown, F.; Cassidy, F.; Clark, M. S. G.; Dabbs, S.; Hadley, M. S.; Hawkins, J.; Loudon, J. M.; Naylor, C. B.; Orlek, B. S.; Riley, G. J. J. Med. Chem. 1997, 40, 4265-4280.
} 
62.8, 62.7, 55.9, 43.0, 33.8, 32.5, 32.3, 29.9, 23.3, 21.3, 21.0, 11.4; CIHRMS [M+H] ${ }^{+}$calculated for $\mathrm{C}_{31} \mathrm{H}_{43} \mathrm{~N}_{2} \mathrm{O}_{11}: 619.2867$, found: 619.2814 .

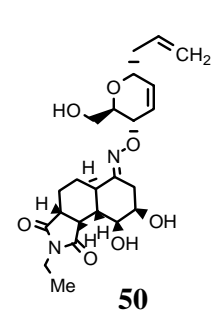

Oxime 50: HPLC-ELSD purity $>99 \%$; $\mathrm{E} / \mathrm{Z}=1: 0\left({ }^{1} \mathrm{H}\right.$ NMR $\left.\left(400 \mathrm{MHz}, \mathrm{CDCl}_{3}\right)\right) ;{ }^{1} \mathrm{H}$ NMR (400 MHz, $\left.\mathrm{CDCl}_{3}\right) \delta$ 5.94-5.80 (m, 4H), 5.16-5.09 (m, 2H), $4.60(\mathrm{~d}, 1 \mathrm{H}, J=10.8$ $\mathrm{Hz}), 4.51-4.46(\mathrm{~m}, 1 \mathrm{H}), 4.27-4.24(\mathrm{~m}, 2 \mathrm{H}), 3.83-3.66(\mathrm{~m}, 3 \mathrm{H}), 3.60-3.51(\mathrm{~m}, 3 \mathrm{H}), 3.32$ $(\mathrm{t}, 1 \mathrm{H}, J=7.2 \mathrm{~Hz}), 2.88-2.82(\mathrm{~m}, 1 \mathrm{H}), 2.50-2.42(\mathrm{~m}, 1 \mathrm{H}), 2.33-2.15(\mathrm{~m}, 3 \mathrm{H}), 1.68-1.61$ $(\mathrm{m}, 1 \mathrm{H}), 1.36-1.25(\mathrm{~m}, 1 \mathrm{H}), 1.18(\mathrm{t}, 3 \mathrm{H}, J=15.2 \mathrm{~Hz}) ;{ }^{13} \mathrm{C} \mathrm{NMR}\left(100 \mathrm{MHz}, \mathrm{CDCl}_{3}\right) \delta$ $179.4,178.5,156.5,134.9,131.3,126.3,117.6,74.2,72.2,71.6,71.4,70.2,63.3,40.8$, $40.4,39.8,39.6,37.9,33.5,30.2,26.1,22.4,13.2$; CIHRMS [M+H] $]^{+}$calculated for $\mathrm{C}_{23} \mathrm{H}_{33} \mathrm{~N}_{2} \mathrm{O}_{7}$ : 449.2288, found: 449.2302 .

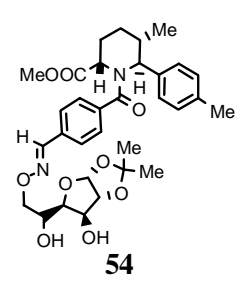

Oxime 54: HPLC-ELSD purity $>99 \%$; $\mathrm{E} / \mathrm{Z}=3.5: 1\left({ }^{1} \mathrm{H}\right.$ NMR $\left.\left(400 \mathrm{MHz}, \mathrm{CDCl}_{3}\right)\right) ;{ }^{1} \mathrm{H}$ NMR (400 MHz, $\mathrm{CDCl}_{3}$, major isomer reported) $\delta 8.07(\mathrm{~s}, 1 \mathrm{H}), 7.48(\mathrm{~d}, 2 \mathrm{H}, J=8.4$ $\mathrm{Hz}), 7.42(\mathrm{~d}, 2 \mathrm{H}, J=8.0 \mathrm{~Hz}), 7.22(\mathrm{~d}, 2 \mathrm{H}, J=8.0 \mathrm{~Hz}), 7.17(\mathrm{~d}, 2 \mathrm{H}, J=8.0 \mathrm{~Hz}), 5.98$ $(\mathrm{d}, 1 \mathrm{H}, J=3.6 \mathrm{~Hz}), 4.67(\mathrm{bs}, 1 \mathrm{H}), 4.54(\mathrm{~d}, 1 \mathrm{H}, J=3.6 \mathrm{~Hz}), 4.45-4.25(\mathrm{~m}, 5 \mathrm{H}), 4.13$ $(\mathrm{dd}, 1 \mathrm{H}, J=2.4,6.0 \mathrm{~Hz}), 3.78(\mathrm{~s}, 3 \mathrm{H}), 3.26-3.24(\mathrm{~m}, 1 \mathrm{H}), 2.34(\mathrm{~s}, 3 \mathrm{H}), 2.23-2.15(\mathrm{~m}$, $1 \mathrm{H}), 1.88-1.83(\mathrm{~m}, 1 \mathrm{H}), 1.74-1.66(\mathrm{~m}, 1 \mathrm{H}), 1.46(\mathrm{~s}, 3 \mathrm{H}), 1.31(\mathrm{~s}, 3 \mathrm{H}), 1.08(\mathrm{~d}, 3 \mathrm{H}, J=$ $6.8 \mathrm{~Hz}) ;{ }^{13} \mathrm{C} \mathrm{NMR}\left(100 \mathrm{MHz}, \mathrm{CDCl}_{3}\right.$, major isomer reported) $\delta 173.9,171.9,149.3,137.7,136.9$, $133.2,129.8,127.9,127.7,127.3,126.4,112.0,105.2,85.3,79.4,75.9,70.6,65.1,55.8,52.5,33.8$, 31.2, 27.0, 26.4, 25.9, 22.3, 21.2, 18.9; CIHRMS [M+H] ${ }^{+}$calculated for $\mathrm{C}_{32} \mathrm{H}_{41} \mathrm{~N}_{2} \mathrm{O}_{9}$ : 597.2812, found: 597.2738 .

4.2 Representative procedure for condensation of alkoxyamines and ketones: Ketone $23 \mathbf{~} 7.9 \mathrm{mg}$, $0.0189 \mathrm{mmol}$, in $1.0 \mathrm{ml}$ EtOAc) was transferred to a microwave reaction vessel using the MiniMapper $^{\mathrm{TM}}$ followed by the addition of alkoxyamine $7(6.8 \mathrm{mg}, 0.0208 \mathrm{mmol}$, in $1.0 \mathrm{ml}$ EtOAc, 1.1 equiv.) and subsequently evaporated using the GeneVac ${ }^{\circledR}$ EZ-2. Upon completion of the evaporation, $\mathrm{AcOH}(0.0038 \mathrm{mmol}$ in $0.2 \mathrm{ml} \mathrm{THF}, 20 \%)$ was added followed by addition of $1 \mathrm{~mL}$ THF. The reaction mixture was subjected to microwave irradiation $\left(100{ }^{\circ} \mathrm{C}, 300 \mathrm{~W}\right)$ for $20 \mathrm{~min}$, concentrated using GeneVac ${ }^{\circledR}$ EZ-2, dissolved in $\mathrm{CH}_{2} \mathrm{Cl}_{2}$, and transferred to a $5 \mathrm{~mL}$ reaction vessel on the Quest 210 synthesizer containing PL-MIA resin $(20 \mathrm{mg}, 2.57 \mathrm{mmol} / \mathrm{g}, 0.051 \mathrm{mmol})$. The scavenging reaction was agitated for $4 \mathrm{~h}$, the filtrate was collected, and the resin was washed with additional $\mathrm{CH}_{2} \mathrm{Cl}_{2}(5$ $\mathrm{mL} \times 2$ ). The combined solution was concentrated on the GeneVac ${ }^{\circledR} \mathrm{EZ}-2$ to afford the desired product (13.3 $\mathrm{mg}, 0.0183 \mathrm{mmol}, 97 \%$ ). The product was dissolved in MeCN and subjected to HPLC-ELSD analysis using the Waters HPLC-MS system $\left(\mathrm{XTerra}^{\circledR} \mathrm{RP}_{8}, 5 \mu \mathrm{m}, 4.6 \times 30 \mathrm{~mm}\right.$ column) to determine product purity. The ratio of $\mathrm{E} / \mathrm{Z}$ oxime isomers was determined by ${ }^{1} \mathrm{H} \mathrm{NMR} .{ }^{19}$

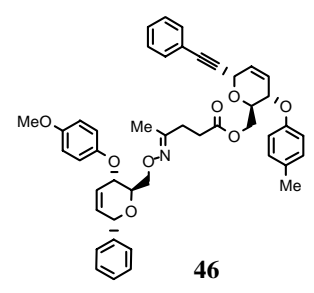

Oxime 46: HPLC-ELSD purity 98.8\%; $\mathrm{E} / \mathrm{Z}=1.5: 1\left({ }^{1} \mathrm{H} \mathrm{NMR}\left(400 \mathrm{MHz}, \mathrm{CDCl}_{3}\right)\right)$; ${ }^{1} \mathrm{H}$ NMR (400 MHz, $\mathrm{CDCl}_{3}$, major isomer reported) $\delta 7.48-7.42(\mathrm{~m}, 4 \mathrm{H}), 7.38-$ $7.29(\mathrm{~m}, 5 \mathrm{H}), 7.11-7.08(\mathrm{~m}, 2 \mathrm{H}), 6.90-6.81(\mathrm{~m}, 5 \mathrm{H}), 6.17-6.14(\mathrm{~m}, 2 \mathrm{H}), 6.05-5.94$ $(\mathrm{m}, 2 \mathrm{H}), 5.35(\mathrm{bs}, 1 \mathrm{H}), 5.24-5.22(\mathrm{~m}, 1 \mathrm{H}), 4.80(\mathrm{~d}, 1 \mathrm{H}, J=6.8 \mathrm{~Hz}), 4.68(\mathrm{t}, 1 \mathrm{H}, J=$ $6.8 \mathrm{~Hz}), 4.53-4.46(\mathrm{~m}, 1 \mathrm{H}), 4.35-4.26(\mathrm{~m}, 3 \mathrm{H}), 4.21-4.16(\mathrm{~m}, 1 \mathrm{H}), 3.99(\mathrm{dt}, 1 \mathrm{H}, J=$ 3.2, 7.6 Hz), 3.77 (s, 3H), 2.54-2.34 (m, 4H), $2.29(\mathrm{~s}, 3 \mathrm{H}), 1.75(\mathrm{~s}, 3 \mathrm{H}) ;{ }^{13} \mathrm{C} \mathrm{NMR}$ $\left(100 \mathrm{MHz}, \mathrm{CDCl}_{3}\right.$, both isomers reported) $\delta 172.9,172.7,157.0,156.0,155.1$, $154.5,151.7,139.6,139.5,132.1,131.2,130.9,130.4,128.9,128.8,128.5,128.13,128.06,126.1$, $125.9,122.4,117.4,115.9,114.9,86.8,85.2,74.1,73.1,71.0,70.5,70.4,65.0,63.9,63.7,31.0,30.5$, 
30.2, 29.9, 25.1, 20.7, 20.2, 14.9; CIHRMS $[\mathrm{M}+\mathrm{H}]^{+}$calculated for $\mathrm{C}_{45} \mathrm{H}_{46} \mathrm{NO}_{8}$ : 728.3223, found: 728.3212 .

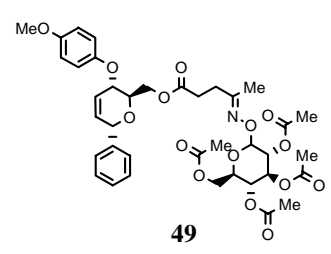

Oxime 49: HPLC-ELSD purity $>99 \%$; $\mathrm{E} / \mathrm{Z}=1: 1\left({ }^{1} \mathrm{H}\right.$ NMR $\left(400 \mathrm{MHz}, \mathrm{CDCl}_{3}\right)$ ); ${ }^{1} \mathrm{H}$ NMR $\left(400 \mathrm{MHz}, \mathrm{CDCl}_{3}\right.$, both isomers reported) $\delta$ 7.44-7.31 (m, 5H), 6.88$6.82(\mathrm{~m}, 4 \mathrm{H}), 6.18(\mathrm{bs}, 2 \mathrm{H}), 5.34(\mathrm{bs}, 1 \mathrm{H}), 5.26-5.22(\mathrm{~m}, 1 \mathrm{H}), 5.17-5.07(\mathrm{~m}, 3 \mathrm{H})$, $4.72(\mathrm{dd}, 1 \mathrm{H}, J=8.0,12.8 \mathrm{~Hz}), 4.32-4.25(\mathrm{~m}, 3 \mathrm{H}), 4.16-4.09(\mathrm{~m}, 1 \mathrm{H}), 3.90-3.86$ (m, $1 \mathrm{H}), 3.80-3.77(\mathrm{~m}, 4 \mathrm{H}), 2.07-1.98(\mathrm{~m}, 12 \mathrm{H}), 1.84(\mathrm{~s}, 1.5 \mathrm{H}), 1.80(\mathrm{~s}, 1.5 \mathrm{H})$; ${ }^{13} \mathrm{C}$ NMR (100 MHz, $\mathrm{CDCl}_{3}$, both isomers reported) $\delta 172.5,172.3,171.0$, $170.5,169.6,169.5,161.2,159.8,154.7,151.3,139.1,130.5,128.7,128.4,128.24,128.17,126.2$, 117.4, 115.0, 102.2, 102.0, 74.5, 74.4, 73.1, 72.1, 70.0, 69.4, 68.3, 68.2, 64.1, 63.9, 61.9, 61.8, 55.9, $30.9,30.2,30.0,25.3,21.0,20.8,20.3,15.4$; CIHRMS $[\mathrm{M}+\mathrm{H}]^{+}$calculated for $\mathrm{C}_{38} \mathrm{H}_{46} \mathrm{NO}_{15}: 756.2867$, found: 756.2952 .

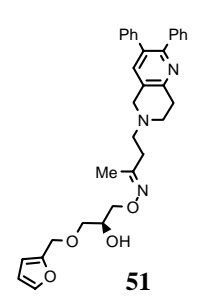

Oxime 51: HPLC-ELSD purity $>99 \%$; E/Z $=3.4: 1\left({ }^{1} \mathrm{H}\right.$ NMR $\left.\left(400 \mathrm{MHz}, \mathrm{CDCl}_{3}\right)\right) ;{ }^{1} \mathrm{H}$ NMR (400 MHz, $\mathrm{CDCl}_{3}$, major isomer reported) $\delta 7.392-7.385(\mathrm{~m}, 2 \mathrm{H}), 7.32-7.29(\mathrm{~m}$, $2 \mathrm{H}), 7.25-7.22(\mathrm{~m}, 5 \mathrm{H}), 7.14-7.12(\mathrm{~m}, 2 \mathrm{H}), 6.34-6.31(\mathrm{~m}, 3 \mathrm{H}), 4.48(\mathrm{~s}, 2 \mathrm{H}), 4.15-4.03(\mathrm{~m}$, $4 \mathrm{H}), 3.88(\mathrm{bs}, 1 \mathrm{H}), 3.55-3.47(3 \mathrm{H}), 3.20(\mathrm{q}, 1 \mathrm{H}, J=5.6 \mathrm{~Hz}), 3.05(\mathrm{bs}, 1 \mathrm{H}), 2.91(\mathrm{bs}, 1 \mathrm{H})$, $2.56(\mathrm{t}, 2 \mathrm{H}, J=7.6 \mathrm{~Hz}), 1.91(\mathrm{~s}, 3 \mathrm{H}) ;{ }^{13} \mathrm{C}$ NMR $\left(100 \mathrm{MHz}, \mathrm{CDCl}_{3}\right.$, major isomer reported) $\delta 157.6,156.8,155.8,151.7,143.0,140.2,139.8,137.0,134.0,130.0,129.7$, $128.4,128.1,127.8,127.2,110.5,109.6,74.4,71.0,70.6,65.3,54.5,53.9,50.8,29.9,20.6,14.8$; CIHRMS $[\mathrm{M}+\mathrm{H}]^{+}$calculated for $\mathrm{C}_{32} \mathrm{H}_{36} \mathrm{~N}_{3} \mathrm{O}_{4}: 526.2706$, found: 526.2755 .

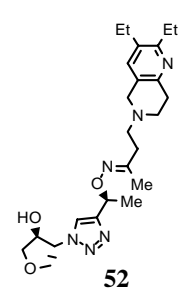

Oxime 52: HPLC-ELSD purity $>99 \%$; $\mathrm{E} / \mathrm{Z}=2.5: 1\left({ }^{1} \mathrm{H}\right.$ NMR $\left.\left(400 \mathrm{MHz}, \mathrm{CD}_{3} \mathrm{OD}\right)\right) ;{ }^{1} \mathrm{H}$ NMR (400 MHz, $\mathrm{CD}_{3} \mathrm{OD}$, major isomer reported) $\delta 7.93(\mathrm{~s}, 1 \mathrm{H}), 7.35(\mathrm{~s}, 1 \mathrm{H}), 5.36(\mathrm{q}, 1 \mathrm{H}$, $J=6.8 \mathrm{~Hz}), 5.03-4.94(\mathrm{~m}, 1 \mathrm{H}), 4.58-4.51(\mathrm{~m}, 1 \mathrm{H}), 4.31-4.02(\mathrm{~m}, 5 \mathrm{H}), 3.90(\mathrm{bs}, 2 \mathrm{H}), 3.77-$ $3.71(\mathrm{~m}, 2 \mathrm{H}), 3.16-2.97(\mathrm{~m}, 4 \mathrm{H}), 2.83(\mathrm{q}, 2 \mathrm{H}, J=8.0 \mathrm{~Hz}), 2.71(\mathrm{q}, 2 \mathrm{H}, J=7.6 \mathrm{~Hz}), 2.60(\mathrm{t}$, $2 \mathrm{H}, J=7.6 \mathrm{~Hz}), 1.89(\mathrm{~s}, 3 \mathrm{H}), 1.61(\mathrm{~d}, 3 \mathrm{H}, J=6.8 \mathrm{~Hz}), 1.25-1.19(\mathrm{~m}, 6 \mathrm{H}) ;{ }^{13} \mathrm{C}$ NMR $(100$ $\mathrm{MHz}, \mathrm{CDCl}_{3}$, both isomers reported) $\delta 166.6,159.8,155.4,150.5,149.6,149.4,136.0$, 135.4, 125.9, 121.5, 120.5, 76.6, 74.7, 74.4, 73.4, 73.0, 71.9, 71.4, 71.0, 68.9, 68.6, 54.1, 52.3, 51.8, $48.3,32.5,29.1,27.2,26.7,24.7,20.1,19.7,19.3,15.0,14.5,14.2$; CIHRMS $[\mathrm{M}+\mathrm{H}]^{+}$calculated for $\mathrm{C}_{24} \mathrm{H}_{37} \mathrm{~N}_{6} \mathrm{O}_{3}: 457.2927$, found: 457.2917 .

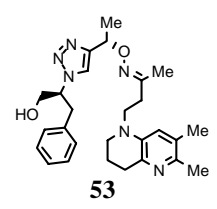

Oxime 53: HPLC-ELSD purity >99\%; E/Z = 2.5:1 ( ${ }^{1} \mathrm{H}$ NMR $\left.\left(400 \mathrm{MHz}, \mathrm{CD}_{3} \mathrm{OD}\right)\right) ;{ }^{1} \mathrm{H}$ NMR $\left(400 \mathrm{MHz}, \mathrm{CD}_{3} \mathrm{OD}\right.$, major isomer reported) $\delta 7.76(\mathrm{~s}, 1 \mathrm{H}), 7.19-7.10(\mathrm{~m}, 3 \mathrm{H})$, $7.04(\mathrm{~d}, 2 \mathrm{H}, J=6.8 \mathrm{~Hz}), 6.87(\mathrm{bs}, 1 \mathrm{H}), 5.26(\mathrm{q}, 1 \mathrm{H}, J=6.8 \mathrm{~Hz}), 4.83-4.80(\mathrm{~m}, 1 \mathrm{H})$, 3.99-3.90 (m, 2H), 3.48-3.42 (m, 2H), 3.27-3.13 (m, 4H), $2.81(\mathrm{t}, 2 \mathrm{H}, J=6.8 \mathrm{~Hz}), 2.41$ $(\mathrm{t}, 2 \mathrm{H}, J=7.6 \mathrm{~Hz}), 2.30(\mathrm{~s}, 3 \mathrm{H}), 2.20(\mathrm{~s}, 3 \mathrm{H}), 1.98-1.92(\mathrm{~m}, 2 \mathrm{H}), 1.86(\mathrm{~s}, 3 \mathrm{H}), 1.54(\mathrm{~d}$, $3 \mathrm{H}, J=6.8 \mathrm{~Hz}) ;{ }^{13} \mathrm{C} \mathrm{NMR}\left(100 \mathrm{MHz}, \mathrm{CDCl}_{3}\right.$, both isomer reported) $\delta 155.6,149.2,141.9,139.8$, $139.5,136.9,130.2,129.2,128.9,127.1,122.3,122.2,120.2,73.5,65.2,65.1,63.7,60.6,48.7,47.8$, $47.1,38.2,32.6,31.8,29.9,29.6,26.9,22.9,21.6,21.0,20.1,19.4,15.3,14.4$; CIHRMS [M+H] ${ }^{+}$ calculated for $\mathrm{C}_{27} \mathrm{H}_{37} \mathrm{~N}_{6} \mathrm{O}_{2}: 477.2978$, found: 477.3038 . 


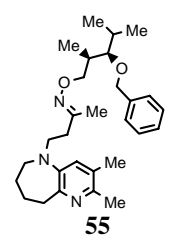

Oxime 55: HPLC-ELSD purity 98.9\%; E/Z = 2.2:1 $\left({ }^{1} \mathrm{H}\right.$ NMR $\left.\left(400 \mathrm{MHz}, \mathrm{CDCl}_{3}\right)\right) ;{ }^{1} \mathrm{H}$ NMR (400 MHz, $\mathrm{CDCl}_{3}$, major isomer reported) $\delta 7.31-7.28(\mathrm{~m}, 5 \mathrm{H}), 6.96(\mathrm{bs}, 1 \mathrm{H}), 4.58$ (bs, $2 \mathrm{H}), 4.08-3.92(\mathrm{~m}, 2 \mathrm{H}), 3.31-3.27(\mathrm{~m}, 2 \mathrm{H}), 3.15-3.08(\mathrm{~m}, 1 \mathrm{H}), 2.98-2.91(\mathrm{~m}, 4 \mathrm{H})$, 2.63-2.60 (m, 1H), $2.46(\mathrm{t}, 1 \mathrm{H}, J=6.8 \mathrm{~Hz}), 2.40(\mathrm{~s}, 3 \mathrm{H}), 2.21-2.14(\mathrm{~m}, 4 \mathrm{H}), 1.95-1.86(\mathrm{~m}$, $4 \mathrm{H}), 1.77-1.60(\mathrm{~m}, 4 \mathrm{H}), 1.03(\mathrm{~d}, 3 \mathrm{H}, J=6.8 \mathrm{~Hz}), 0.95(\mathrm{~d}, 3 \mathrm{H}, J=6.4 \mathrm{~Hz}), 0.90(\mathrm{~d}, 3 \mathrm{H}, J=$ $6.8 \mathrm{~Hz}) ;{ }^{13} \mathrm{C}$ NMR $\left(100 \mathrm{MHz}, \mathrm{CDCl}_{3}\right.$, both isomers reported) $\delta 156.4,156.1,153.7,153.2,148.1$, 148.0, 145.6, 145.5, 145.48, 139.4, 129.1, 128.5, 127.8, 127.7, 127.6, 127.1, 126.9, 85.5, 85.4, 76.6, 76.5, 75.2, 54.4, 54.2, 37.0, 35.4, 35.2, 31.3, 30.4, 30.3, 29.9, 29.1, 24.5, 21.7, 20.7, 19.9, 19.7, 19.3, 14.5, 11.14, 11.06; CIHRMS [M+H] calculated for $\mathrm{C}_{29} \mathrm{H}_{44} \mathrm{~N}_{3} \mathrm{O}_{2}$ : 466.3433, found: 466.3464 .

4.3 Representative procedure for condensation of alkoxyamines and ketone 30: Ketone 30 (5.7 $\mathrm{mg}, 0.0189 \mathrm{mmol}$, in $1 \mathrm{ml}$ EtOAc) was transferred to reaction vessel using MiniMapper ${ }^{\mathrm{TM}}$ followed by addition of alkoxyamine $14(7.4 \mathrm{mg}, 0.0284 \mathrm{mmol}$, in $1.37 \mathrm{ml} \mathrm{EtOAc}, 1.5$ equiv.) and then evaporated using the GeneVac ${ }^{\circledR}$ EZ-2. Upon the completion of evaporation, PPTS $(1.0 \mathrm{mg}, 0.0038 \mathrm{mmol}, 20 \%)$ was added followed by addition of $1 \mathrm{~mL}$ of THF. The reaction mixture was subjected to microwave irradiation $\left(110^{\circ} \mathrm{C}, 300 \mathrm{~W}\right)$ for $45 \mathrm{~min}$, concentrated, dissolved in $\mathrm{CH}_{2} \mathrm{Cl}_{2}$, and applied to an SLE cartridge $(5.0 \mathrm{~mL})$ containing sat. $\mathrm{NaHCO}_{3}$. After eluting with $15 \mathrm{~mL} \mathrm{CH}_{2} \mathrm{Cl}_{2}$, the elutant was concentrated, dissolved in $\mathrm{CH}_{2} \mathrm{Cl}_{2}$, and transferred to a $5 \mathrm{~mL}$ reaction vessel on Quest 210 synthesizer containing PL-MIA resin $(20 \mathrm{mg}, 2.57 \mathrm{mmol} / \mathrm{g}, 0.051 \mathrm{mmol})$. The scavenging reaction was agitated for $4 \mathrm{~h}$, the filtrate was collected, and the resin was washed with additional $\mathrm{CH}_{2} \mathrm{Cl}_{2}(5 \mathrm{~mL} \times 2)$. The combined solution was concentrated on the GeneVac ${ }^{\circledR}$ EZ-2 to afford the desired product $(9.7 \mathrm{mg}$, $0.178 \mathrm{mmol}, 94 \%)$. The product was dissolved in MeCN and subjected to HPLC-ELSD analysis using a Waters HPLC-MS system (XTerra ${ }^{\circledR R P} P_{8}, 5 \mu \mathrm{m}, 4.6 \times 30 \mathrm{~mm}$ column) to determine product purity. The ratio of $\mathrm{E} / \mathrm{Z}$ oxime isomers was determined by ${ }^{1} \mathrm{H}$ NMR. ${ }^{19}$

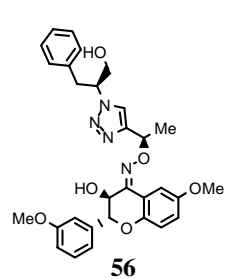

Oxime 56: HPLC-ELSD purity 98.9\%; E/Z = 1.2:1 ( ${ }^{1} \mathrm{H}$ NMR $\left.\left(400 \mathrm{MHz}, \mathrm{CDCl}_{3}\right)\right) ;{ }^{1} \mathrm{H}$ NMR (400 MHz, $\mathrm{CDCl}_{3}$, major isomer reported) $\delta 7.34(\mathrm{t}, 1 \mathrm{H}, J=8.0 \mathrm{~Hz}), 7.27-7.12$ $(\mathrm{m}, 5 \mathrm{H}), 7.06-6.83(\mathrm{~m}, 7 \mathrm{H}), 5.51(\mathrm{q}, 1 \mathrm{H}, J=6.4 \mathrm{~Hz}), 5.16(\mathrm{~d}, 1 \mathrm{H}, J=7.2 \mathrm{~Hz}), 4.92(\mathrm{~d}$, $1 \mathrm{H}, J=6.0 \mathrm{~Hz}), 4.64-4.62(\mathrm{~m}, 1 \mathrm{H}), 4.17-4.02(\mathrm{~m}, 2 \mathrm{H}), 3.81(\mathrm{~s}, 3 \mathrm{H}), 3.76(\mathrm{~s}, 3 \mathrm{H}), 3.26-$ $3.15(\mathrm{~m}, 2 \mathrm{H}), 1.59(\mathrm{~d}, 3 \mathrm{H}, J=6.4 \mathrm{~Hz}) ;{ }^{13} \mathrm{C} \mathrm{NMR}\left(100 \mathrm{MHz}, \mathrm{CDCl}_{3}\right.$, both isomer reported) $\delta 159.8,154.6,153.5,151.7,150.5,150.2,148.0,147.0,139.4,139.0,136.7$, 136.6, 129.7, 129.2, 128.9, 127.3, 122.7, 122.5, 120.2, 120.0, 119.5, 119.1, 118.4, 118.3, 114.5, 114.1, $113.1,112.8,107.3,82.3,81.2,75.6,75.0,69.7,66.7,65.4,65.2,63.8,63.7,56.0,55.9,55.5,55.4$, 38.1, 29.9, 29.7, 20.1, 19.6; CIHRMS [M+H] $]^{+}$calculated for $\mathrm{C}_{30} \mathrm{H}_{33} \mathrm{~N}_{4} \mathrm{O}_{6}: 545.2400$, found: 545.2448.

4.4 Representative procedure for preparation of acetone oximes: Alkoxyamine 7 ( $6.8 \mathrm{mg}, 0.0208$ мео ме ${ }_{\text {ме }} \mathrm{mmol}$, in $1.0 \mathrm{ml} \mathrm{EtOAc)}$ was transferred to a reaction vessel using the MiniMapper $^{\mathrm{TM}}$ followed by the addition of $2 \mathrm{~mL}$ acetone. The reaction was stirred at $\mathrm{rt}$ for $1 \mathrm{~h}$ and concentrated on the GeneVac ${ }^{\circledR}$ EZ-2 to afford the desired product $(7.6 \mathrm{mg}, 0.0208$ mmol, $100 \%$ ). The product was dissolved in MeCN and subjected to HPLC-ELSD analysis using the Waters HPLC-MS system $\left(\mathrm{XTerra}^{\circledR R} \mathrm{R}_{8}, 5 \mu \mathrm{m}, 4.6 \times 30 \mathrm{~mm}\right.$ column$)$ to determine product purity. 


\subsection{Representative procedure for condensation of $O$-methyl hydroxylamine and}

aldehydes/ketones: Ketone $23(7.9 \mathrm{mg}$, $0.0189 \mathrm{mmol}$, in $1.0 \mathrm{ml}$ EtOAc) was transfer to reaction vessel using MiniMapper ${ }^{\mathrm{TM}}$ followed by the addition of $O$-methyl hydroxylamine ( $1.8 \mathrm{mg}, 0.0378 \mathrm{mmol}$, in $1.0 \mathrm{ml} \mathrm{EtOAc,} 2.0$ equiv.) and $\mathrm{AcOH}(0.0038$ mmol in $0.2 \mathrm{ml} \mathrm{EtOAc,} \mathrm{20 \% ).} \mathrm{The} \mathrm{reaction} \mathrm{mixture} \mathrm{was} \mathrm{stirred} \mathrm{at} \mathrm{rt}$ for $12 \mathrm{~h}$, and concentrated using the GeneVac ${ }^{\mathbb{R}}$ EZ-2 to afford the desired product $(8.5 \mathrm{mg}, 0.0189$ mmol, $100 \%$ ). The product was dissolved in MeCN and subjected to HPLC-ELSD analysis using a Waters HPLC-MS system (XTerra ${ }^{\circledR} R_{8}, 5 \mu \mathrm{m}, 4.6 \times 30 \mathrm{~mm}$ column) to determine product purity. For ketone 29, 1.1 equiv of $O$-methyl hydroxylamine was used. For ketone 28, reaction was carried out in $3: 1$ (volume) $\mathrm{CH}_{2} \mathrm{Cl}_{2}: O$-methyl hydroxylamine (20 mmol\% PPTS) under the irradiation of microwave $\left(100{ }^{\circ} \mathrm{C}, 150 \mathrm{~W}, 20 \mathrm{~min}\right)$. 


\section{Experimental Procedure for Biological Assays}

\subsection{Cells and Cell Culture Conditions}

A549 cells were cultured in Dulbecco's modified Eagle's medium (DMEM) supplemented with $10 \%$ fetal bovine serum (FBS), $0.1 \%$ glutamine, and $0.1 \%$ penicillin-streptomycin at $37^{\circ} \mathrm{C}$ in a humidified atmosphere of $5 \% \mathrm{CO}_{2}$.

\subsection{Cell Growth Inhibition Assay}

Exponentially growing cells were diluted to a concentration of $6 \times 10^{4}$ cells $/ \mathrm{ml}$ and seeded in 96-well flat-bottom microtiter plates $(50 \mu \mathrm{l} /$ well $)$. The assay plates were incubated for 24 hours at $37^{\circ} \mathrm{C}$ in a humidified atmosphere of $5 \% \mathrm{CO}_{2}$. The library was dissolved in dimethyl sulfoxide to a concentration of $0.5 \mathrm{mM}$. The stock solutions were further diluted in media to $0.02 \mathrm{mM}$. Fifty microliters of the diluted compounds were transferred to the assay plates resulting in a treatment concentration of $10 \mu \mathrm{M}$. The cell cultures were incubated with the compounds for 48 hours at $37^{\circ} \mathrm{C}$ in a humidified atmosphere of $5 \% \mathrm{CO}_{2}$. Cell proliferation was quantified using the CellTiter $96^{\circledR} \mathrm{AQ}_{\text {ueous }}$ One Solution Cell Proliferation Assay (Promega Corporation). The assay solution contains Owen's reagent, MTS: 3(4,5-dimethylthiazol-2-yl)-5-(3-carboxymethoxyphenyl)-2-(4-sulfophenyl)-2H-tetrazolium inner salt, ${ }^{20}$ and an electron coupling reagent, phenazine ethosulfate. Twenty microliters of the CellTiter $96^{\circledR}$ $\mathrm{AQ}_{\text {ueous }}$ One Solution Reagent were added to the wells, and the cultures were incubated for one hour at $37{ }^{\circ} \mathrm{C}$ in a humidified atmosphere of $5 \% \mathrm{CO}_{2}$. The absorbance of the wells was measured at $490 \mathrm{~nm}$ using a microplate reader to monitor the formation of the formazan product resulting from the bioreduction of the MTS tetrazolium compound by metabolically active cells to determine cell viability. After the initial library screen, the procedure was repeated using serial dilutions of the lead compounds to determine $\mathrm{IC}_{50}$ values. The final concentration of the compounds ranged from $40 \mu \mathrm{M}$ to $78 \mathrm{nM}$. The $\mathrm{IC}_{50}$ values were calculated using the least squares method. Each assay was run in triplicate.

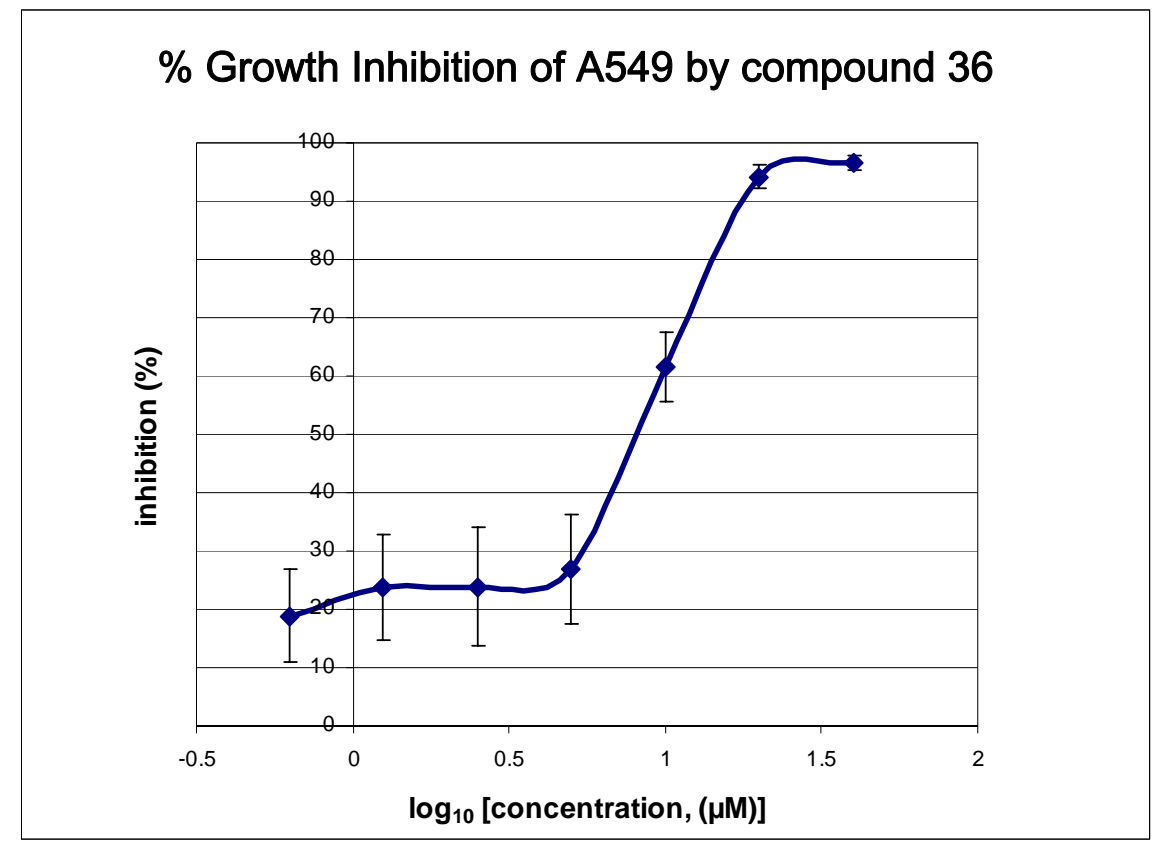

Figure 3. Growth inhibition curve (\%) of A549 treated with compound 36.

${ }^{20}$ Barltrop, J. A.; Owen, T. C.; Cory, A. H.; Cory, J. G. Bioorg. Med. Chem. Lett. 1991, 1, 611-614. 


\section{Representative ${ }^{1} \mathrm{H}$ and ${ }^{13} \mathrm{C}$ NMR Spectra ${ }^{21}$}

18
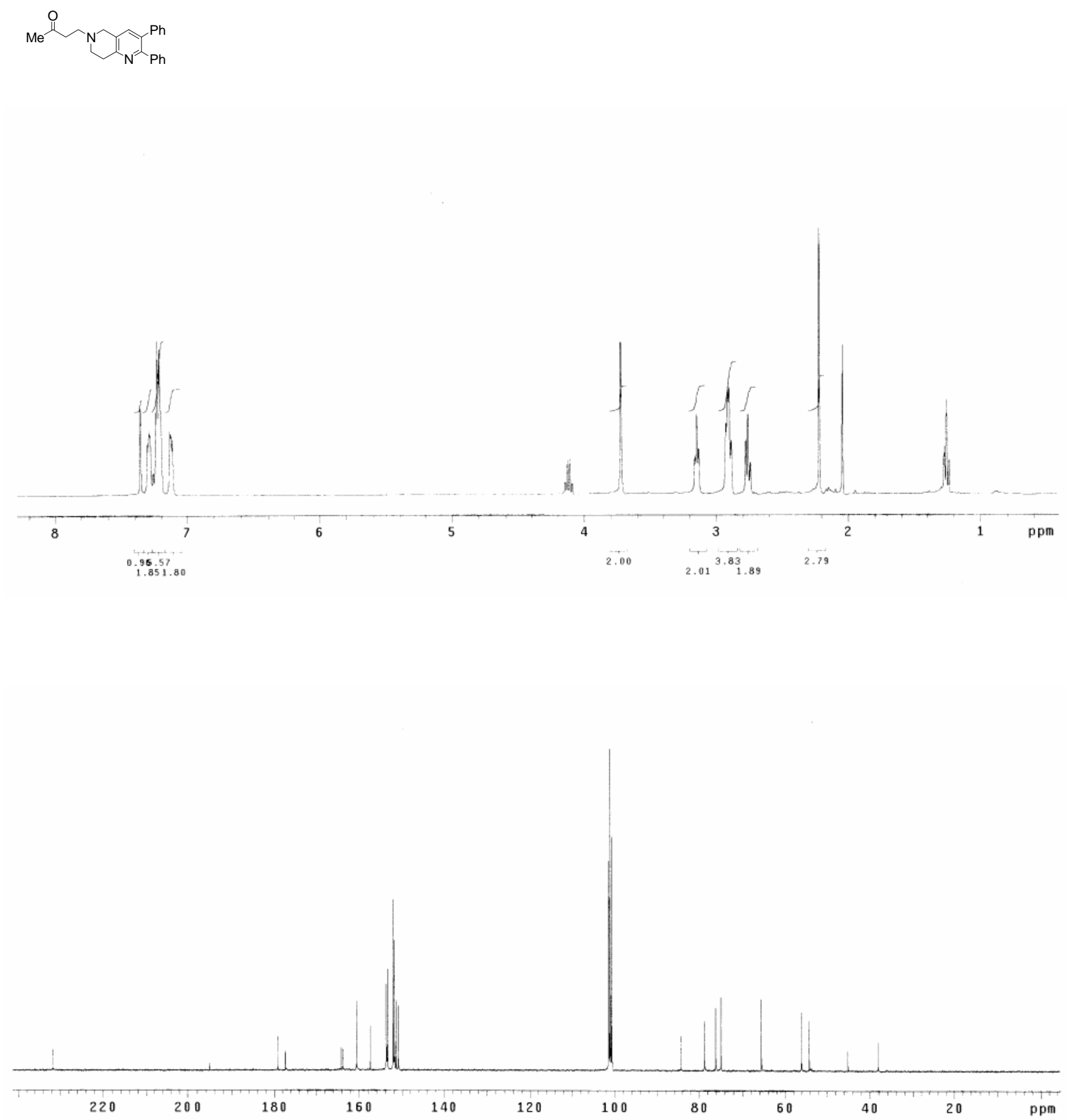

${ }^{21} \mathrm{H}(400 \mathrm{MHz})$ and ${ }^{13} \mathrm{C}$ NMR $(100 \mathrm{MHz})$ spectra were obtained in $\mathrm{CDCl}_{3}$ unless otherwise indicated. 


$$
\overbrace{}^{\text {ONt }}
$$
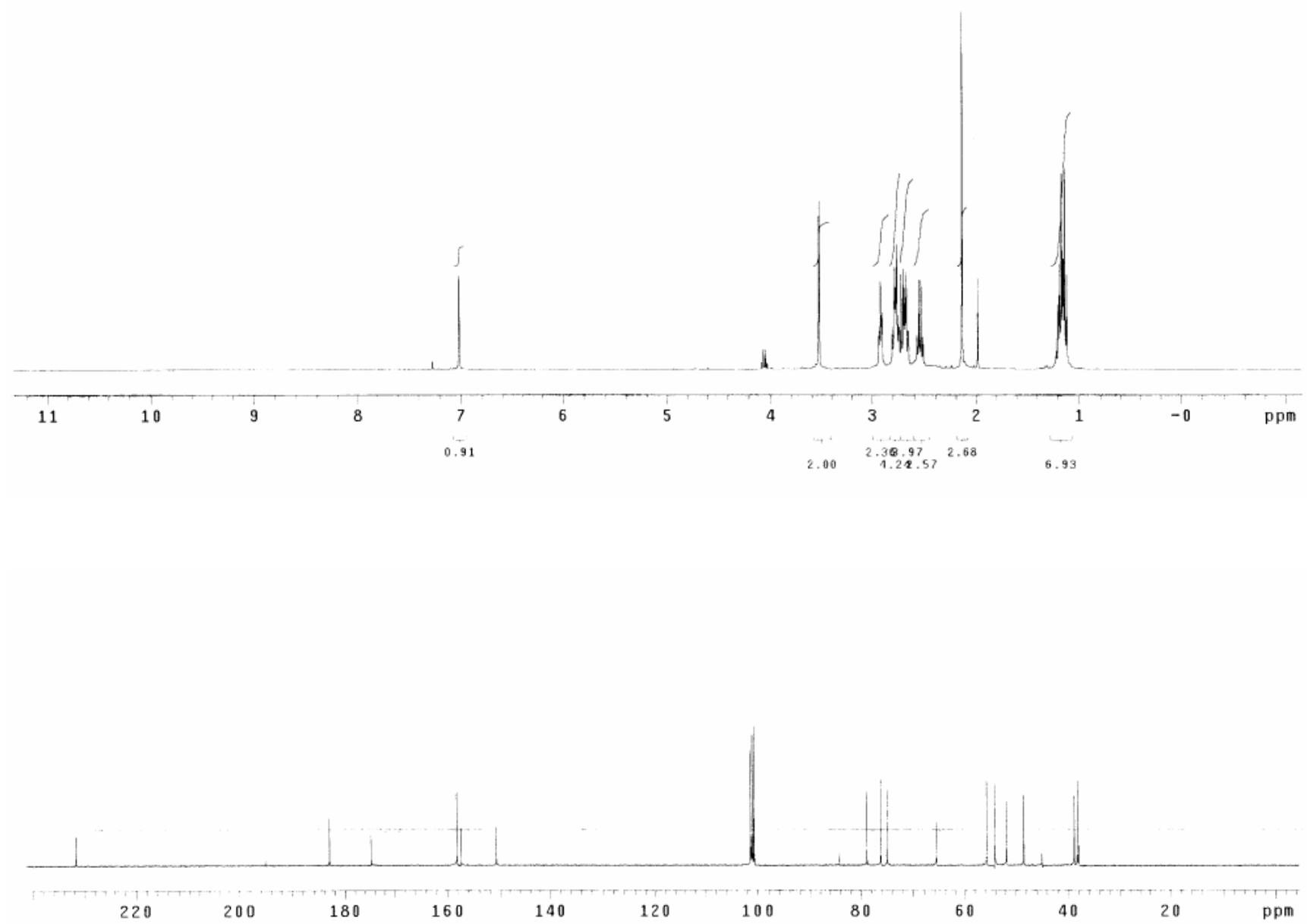
Me
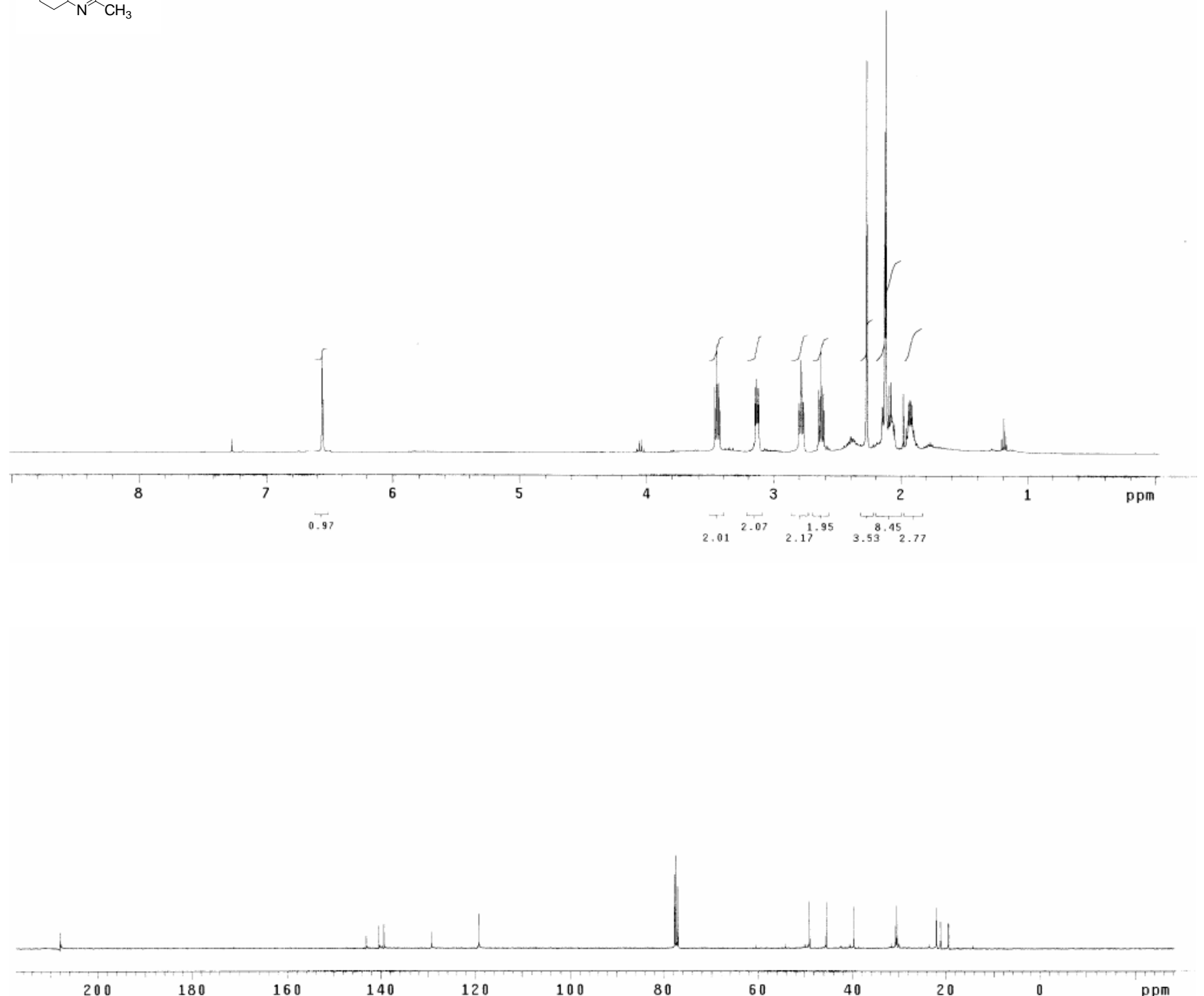
21

Oye
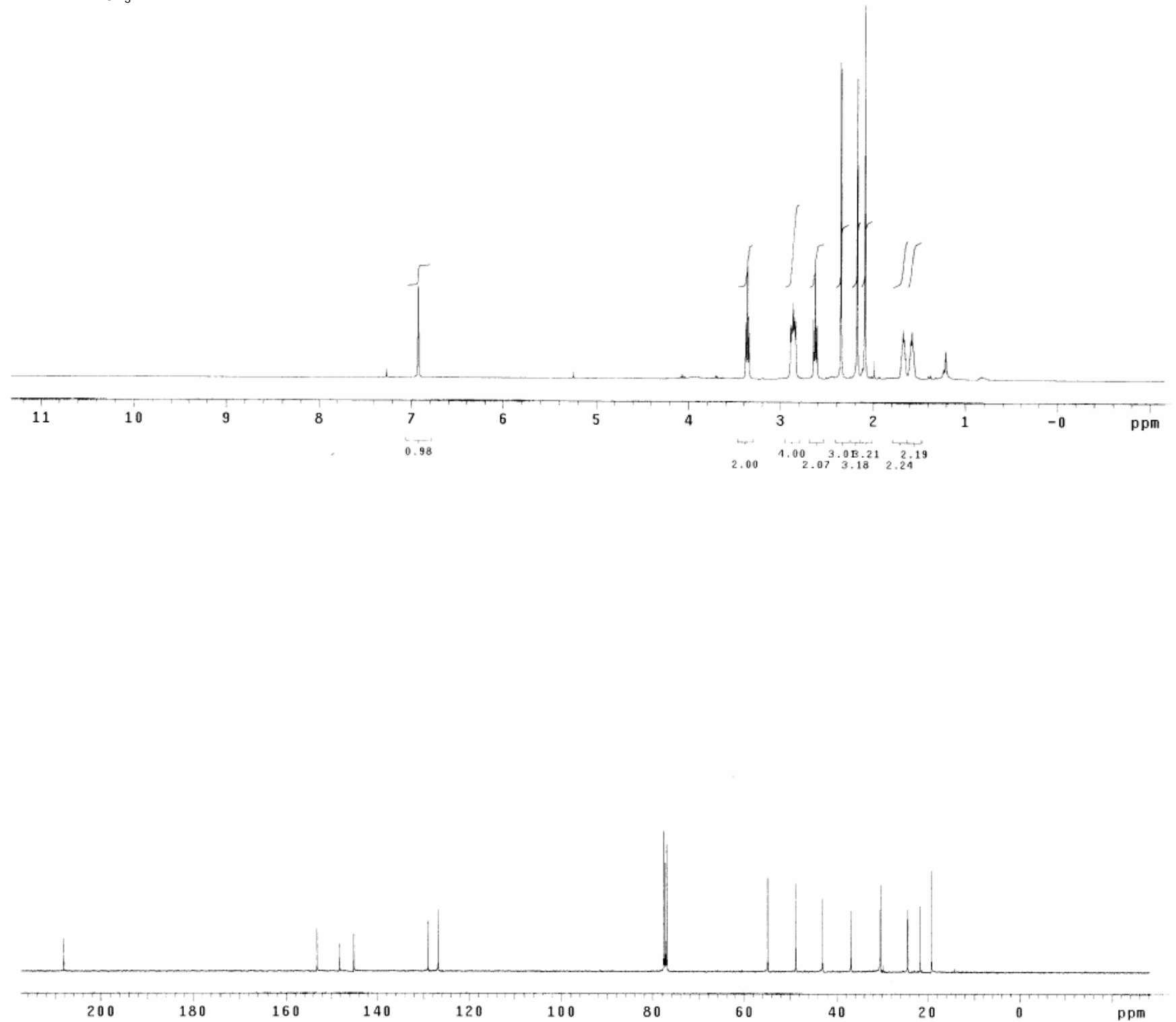

- S 34 - 

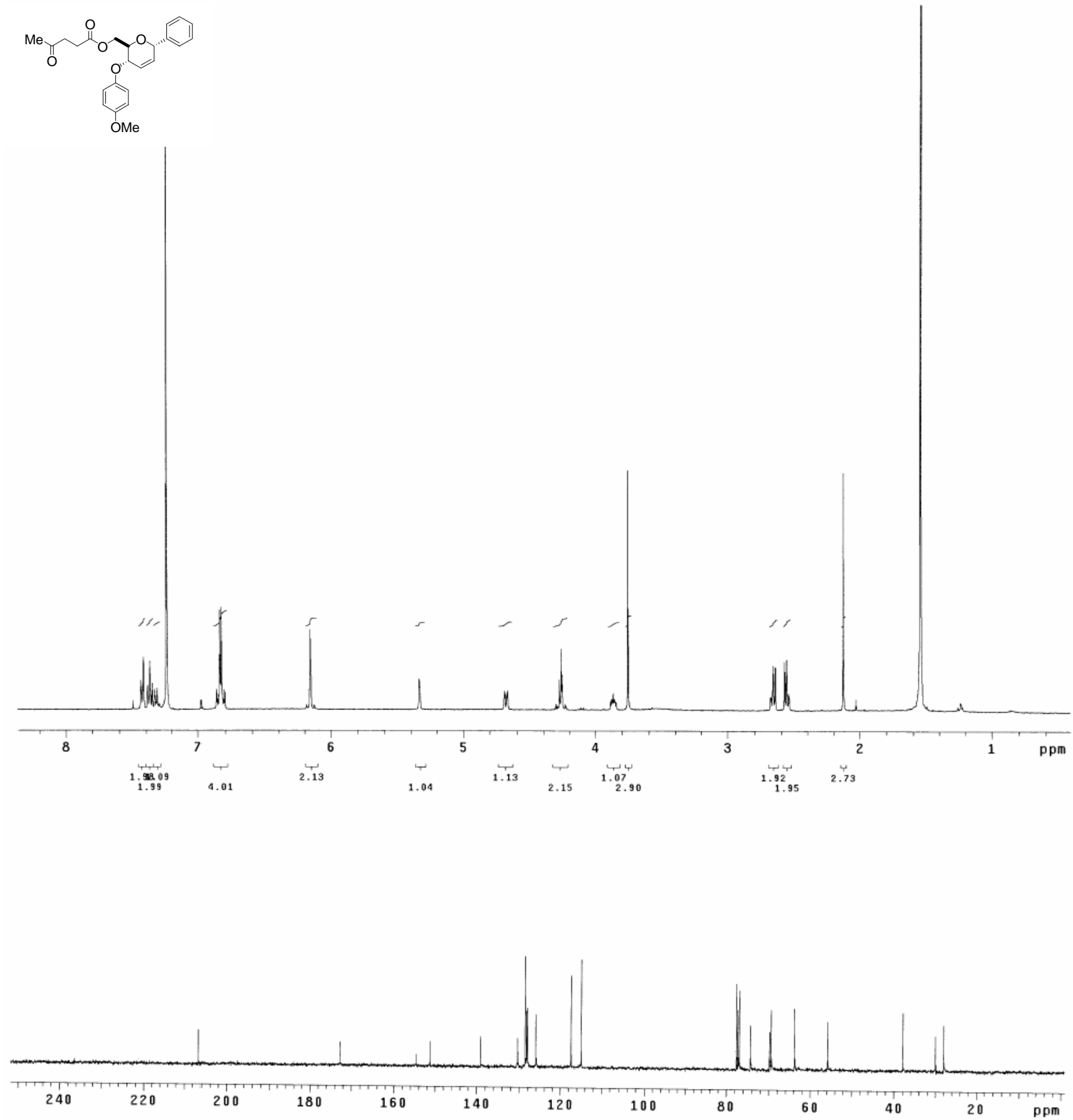

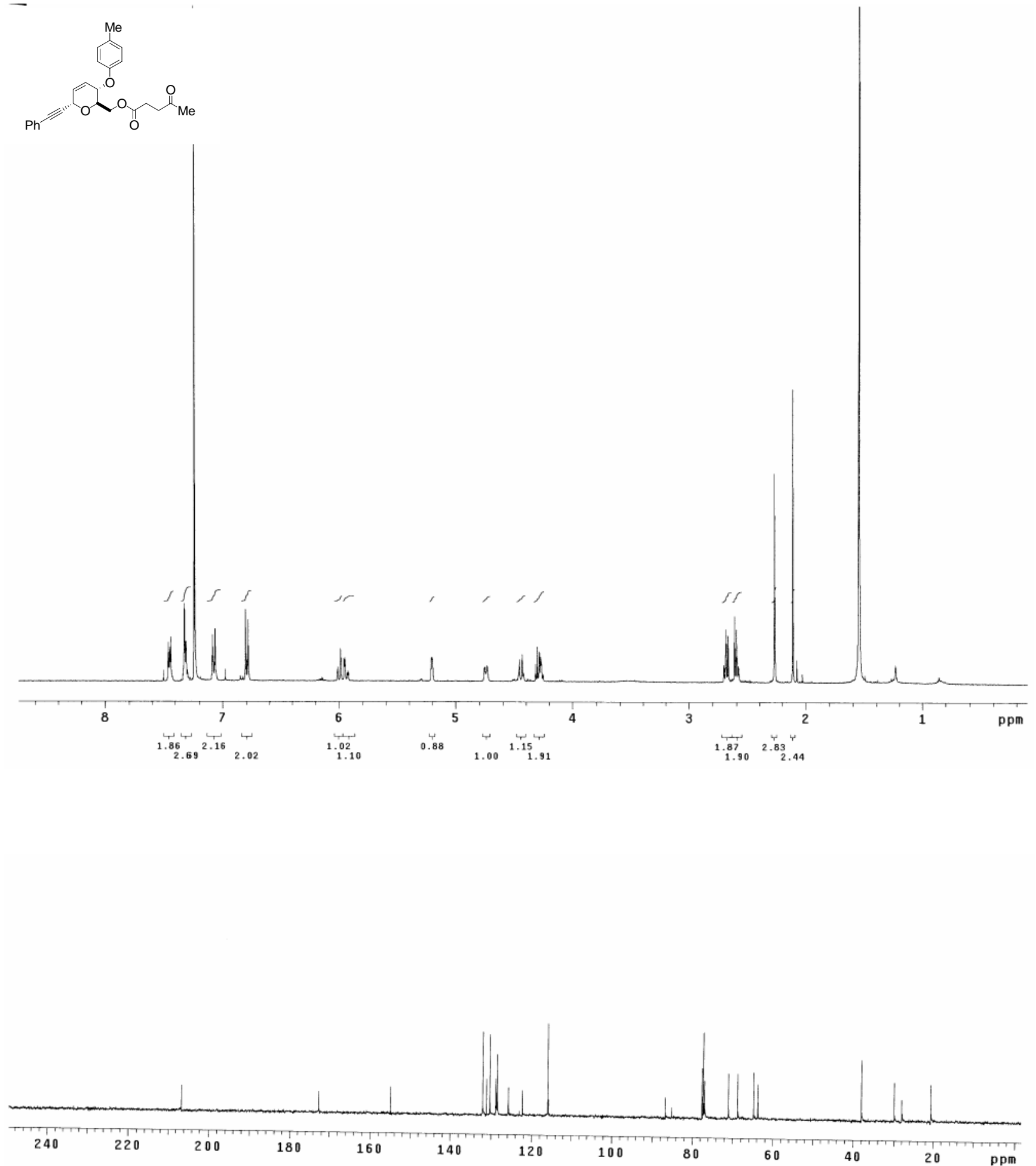
41
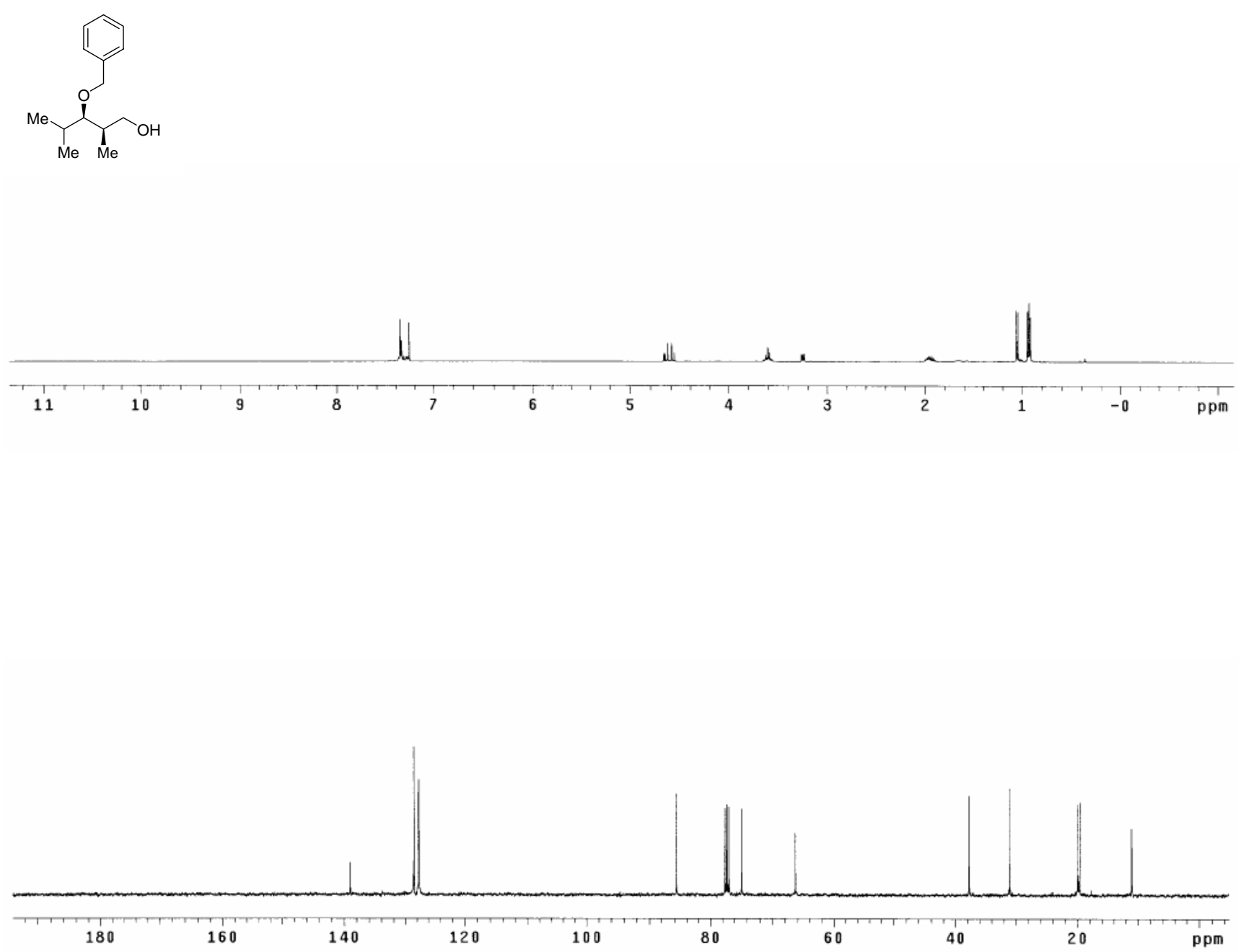
28

(n)
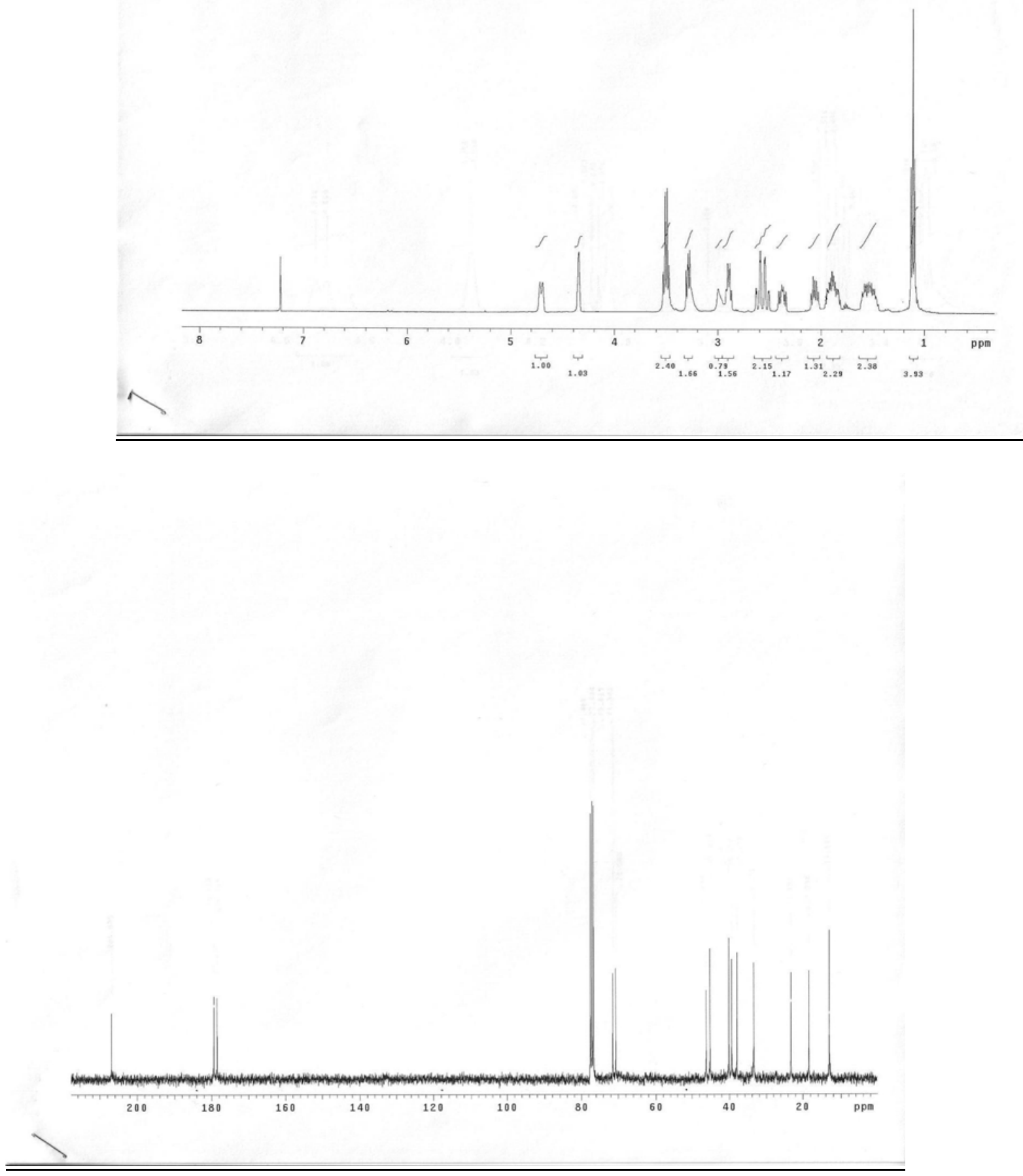

- S 38 - 
$>^{N-N_{O}^{-1}}$

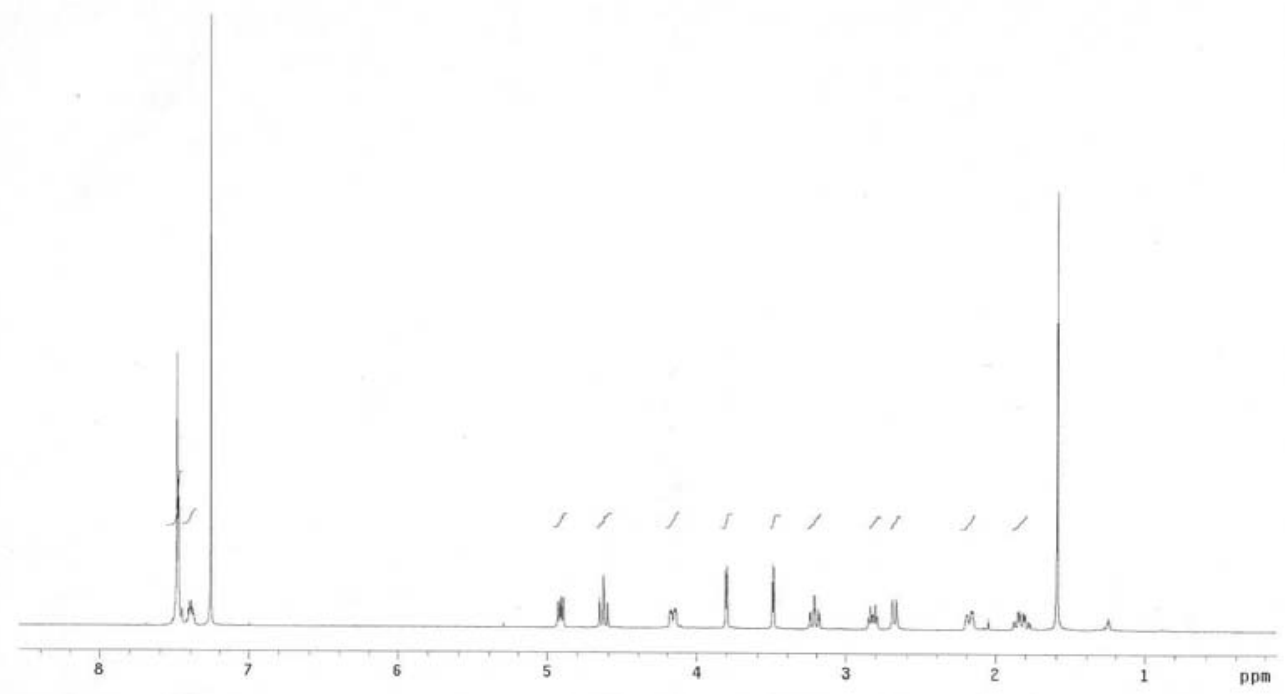

$>$

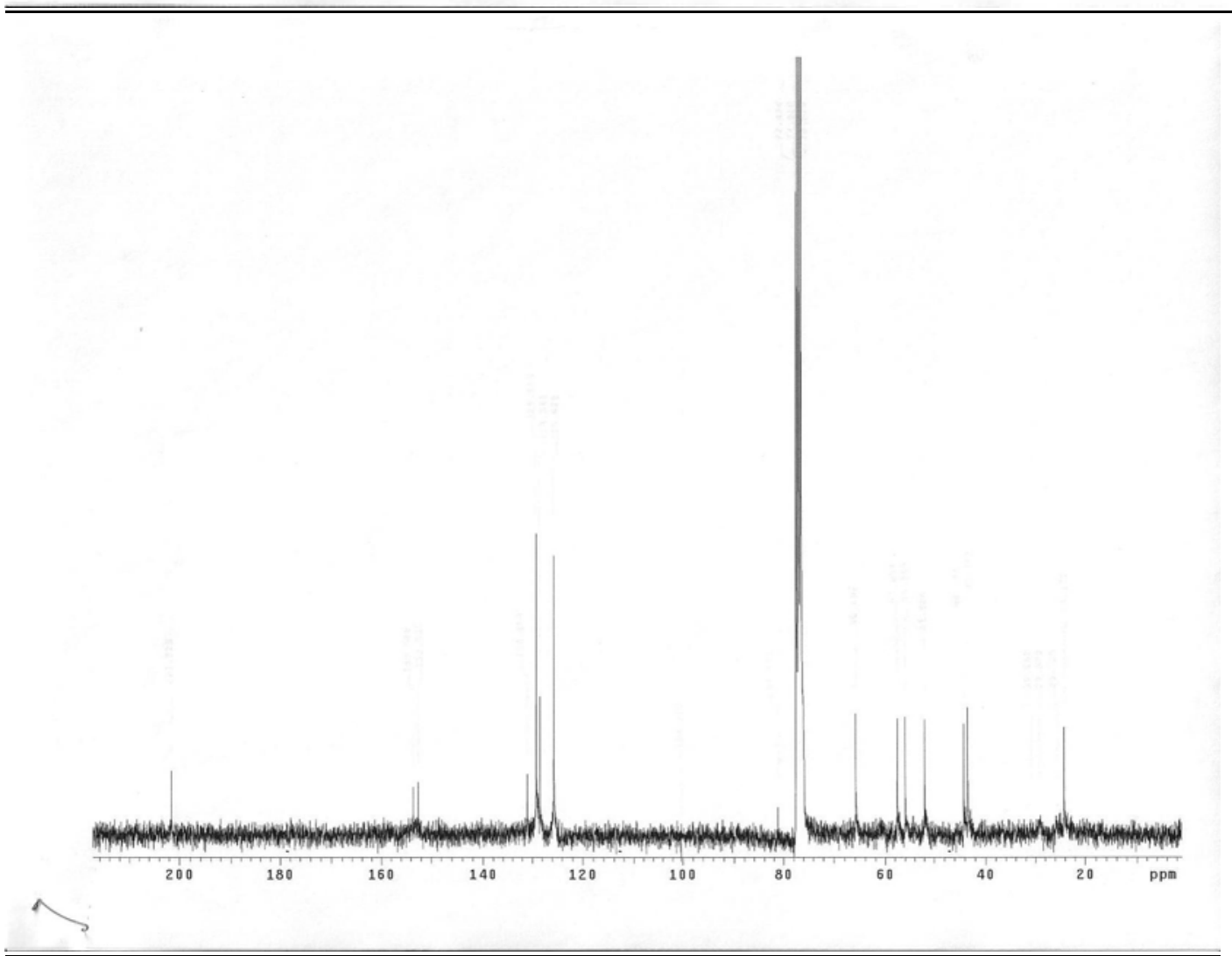

- S 39 - 
MeO
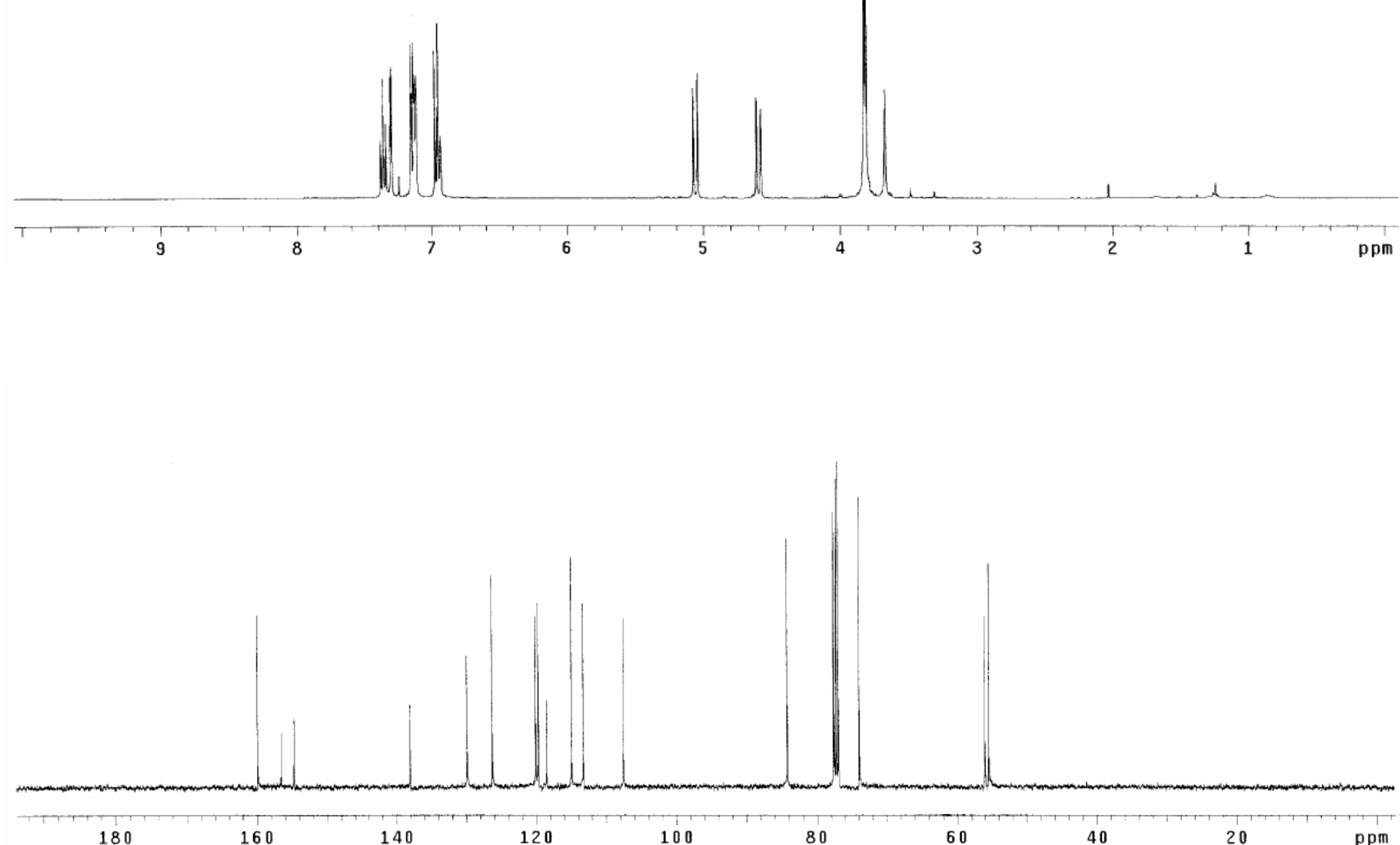

- S 40 - 

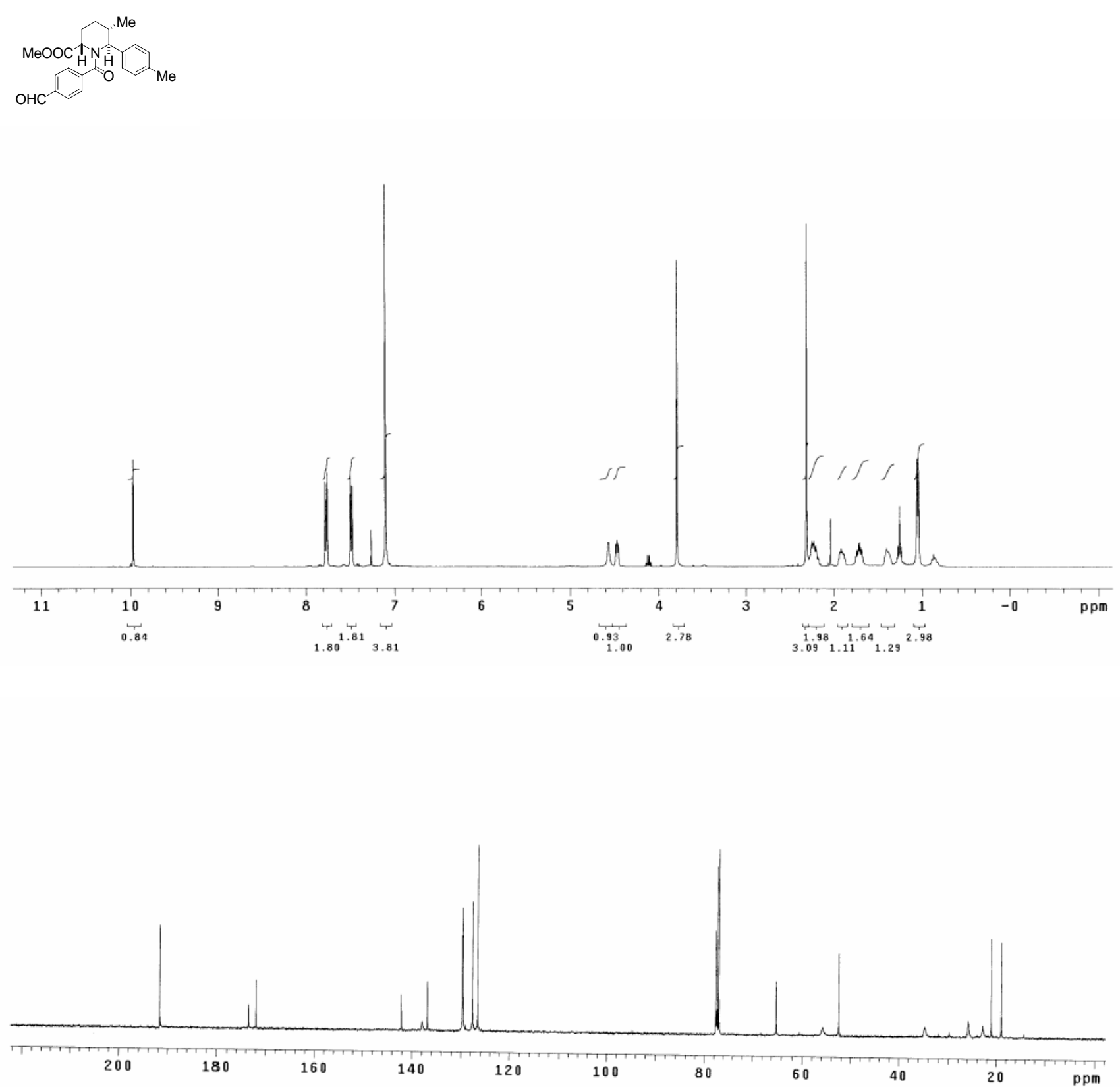


\section{Phth-6}
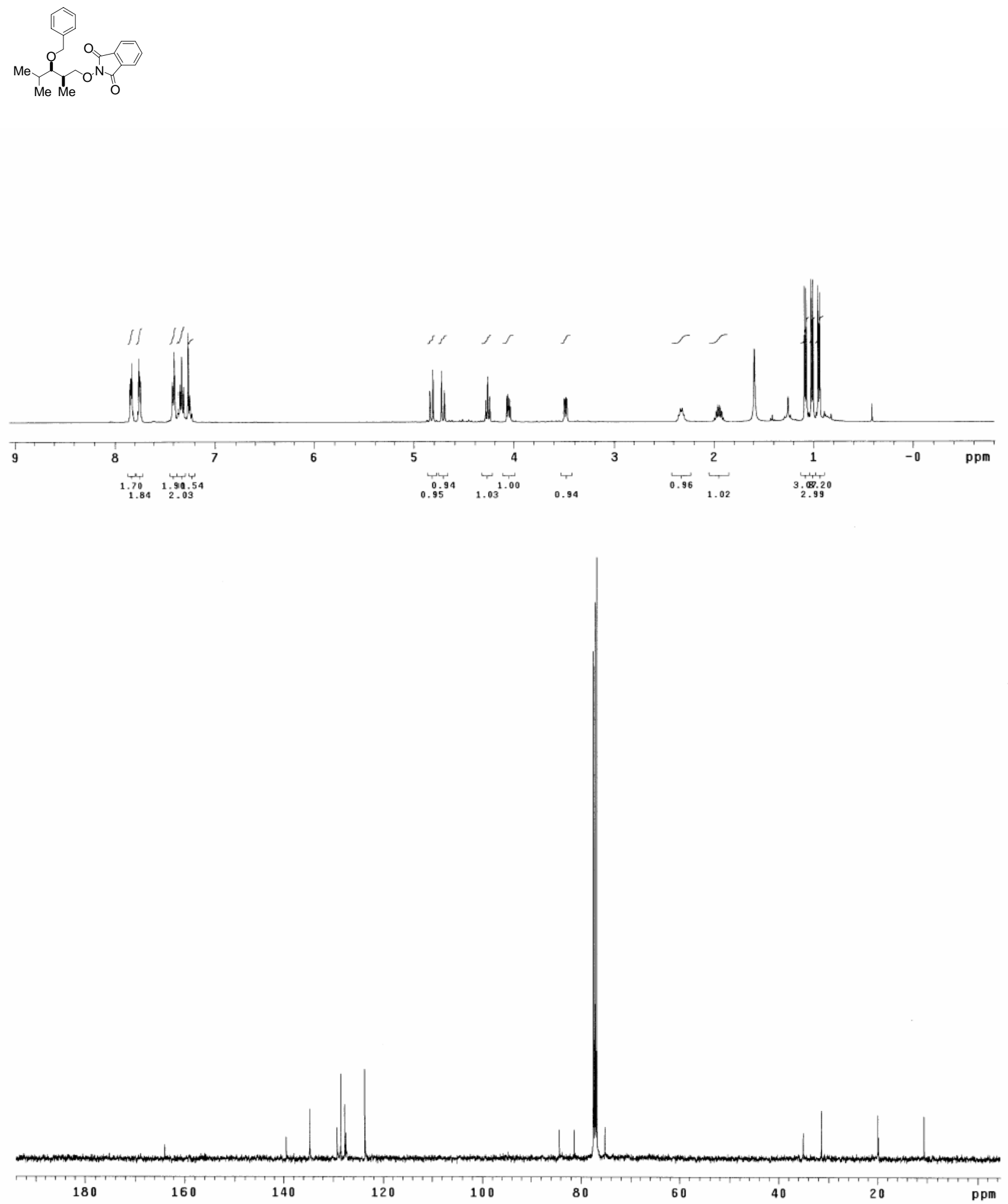
Phth-7
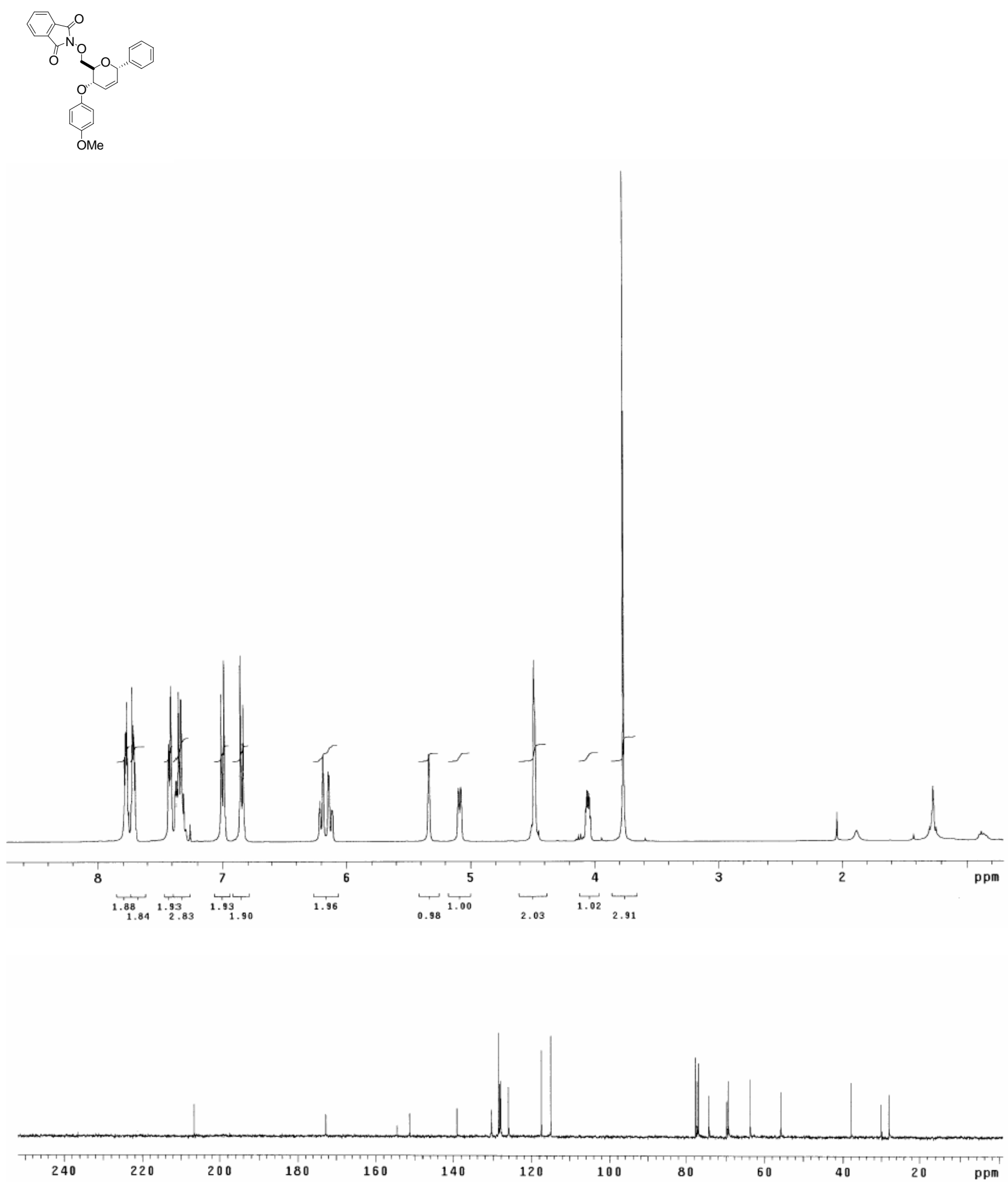


\section{Phth-8}
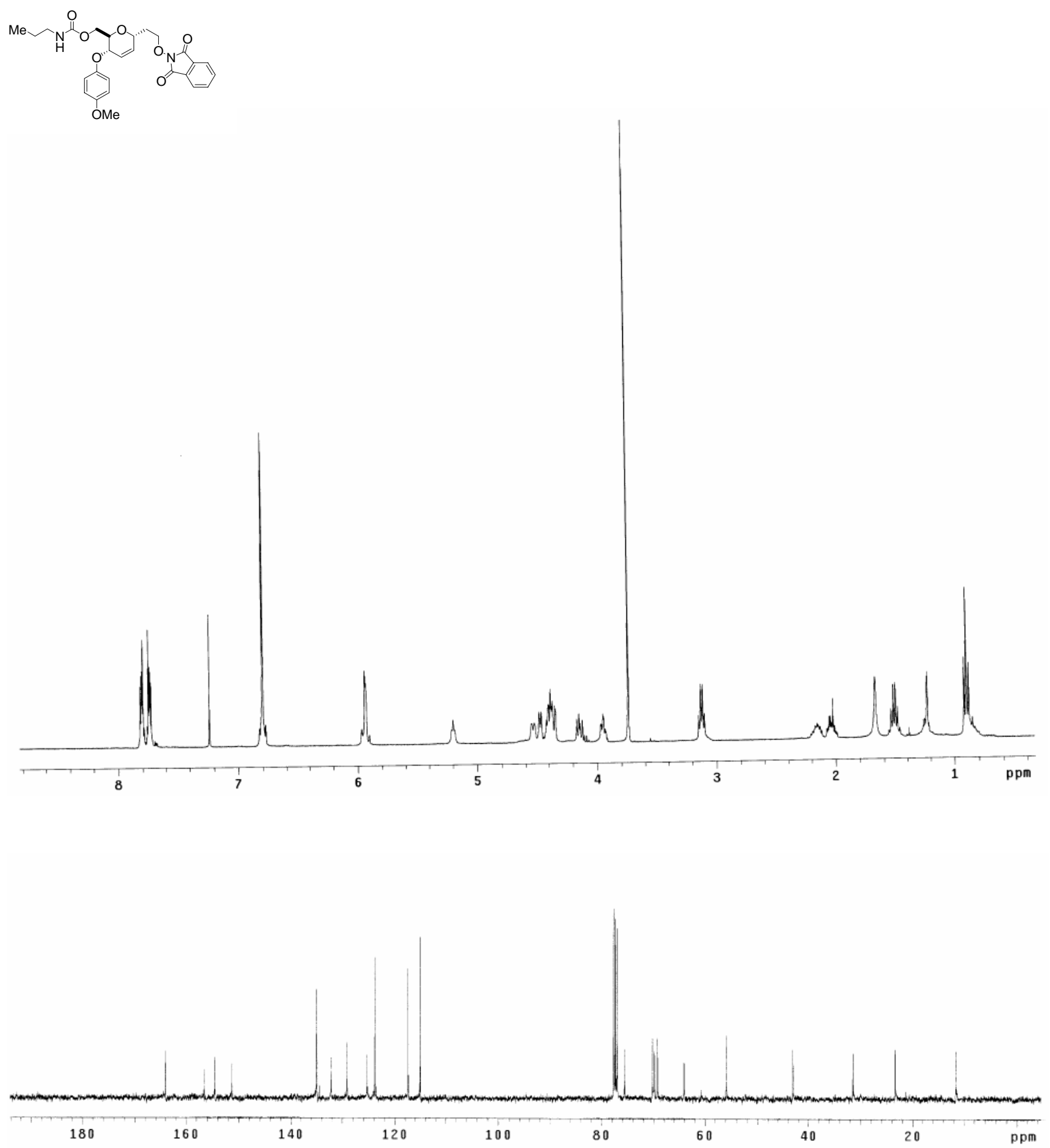
Phth-9

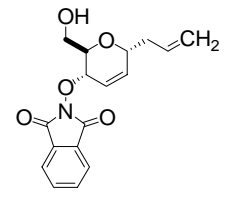
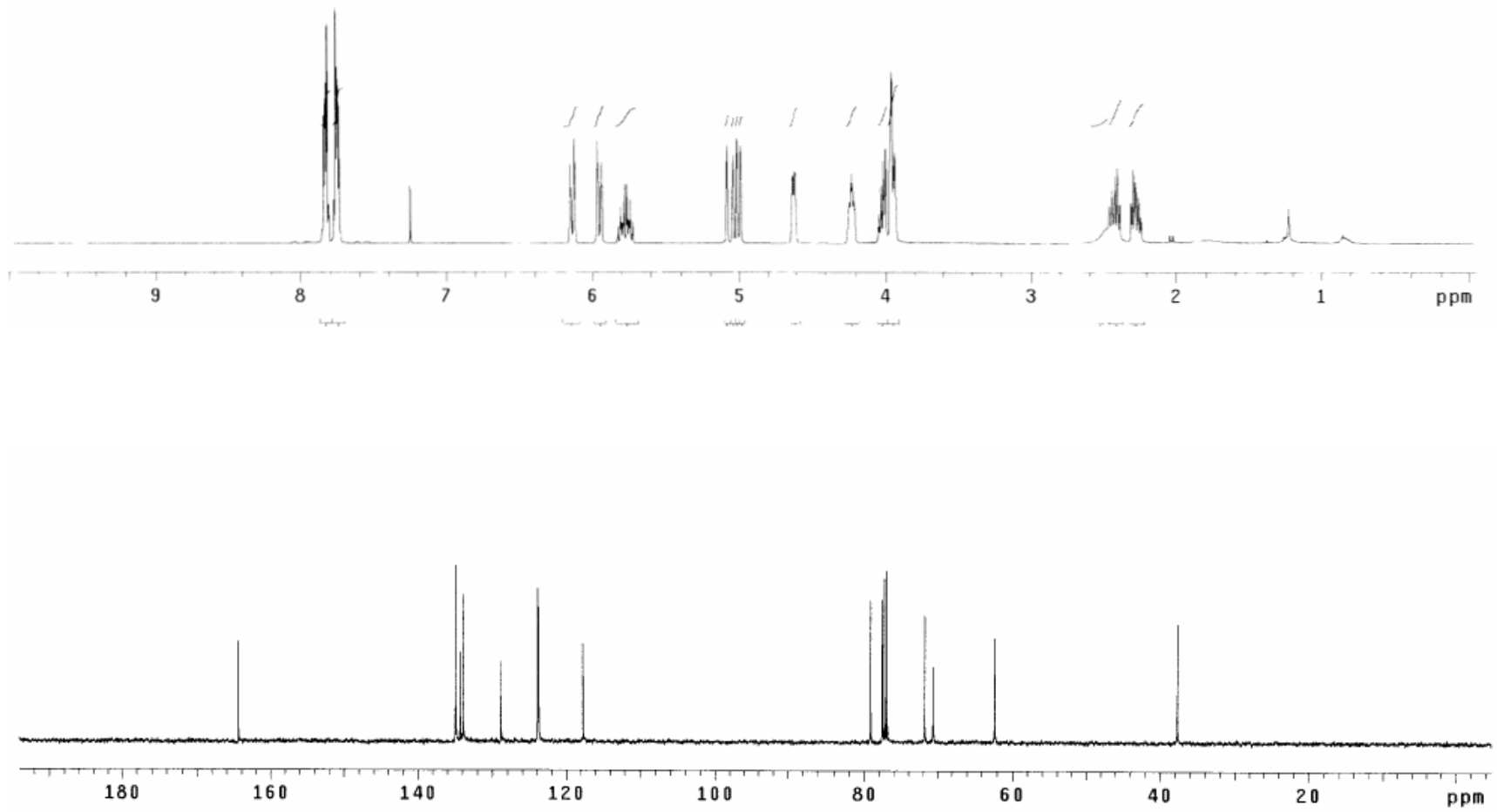


\section{Phth-10}

${ }_{\mathrm{AcO}}^{\mathrm{AcO}}>\mathrm{O}$
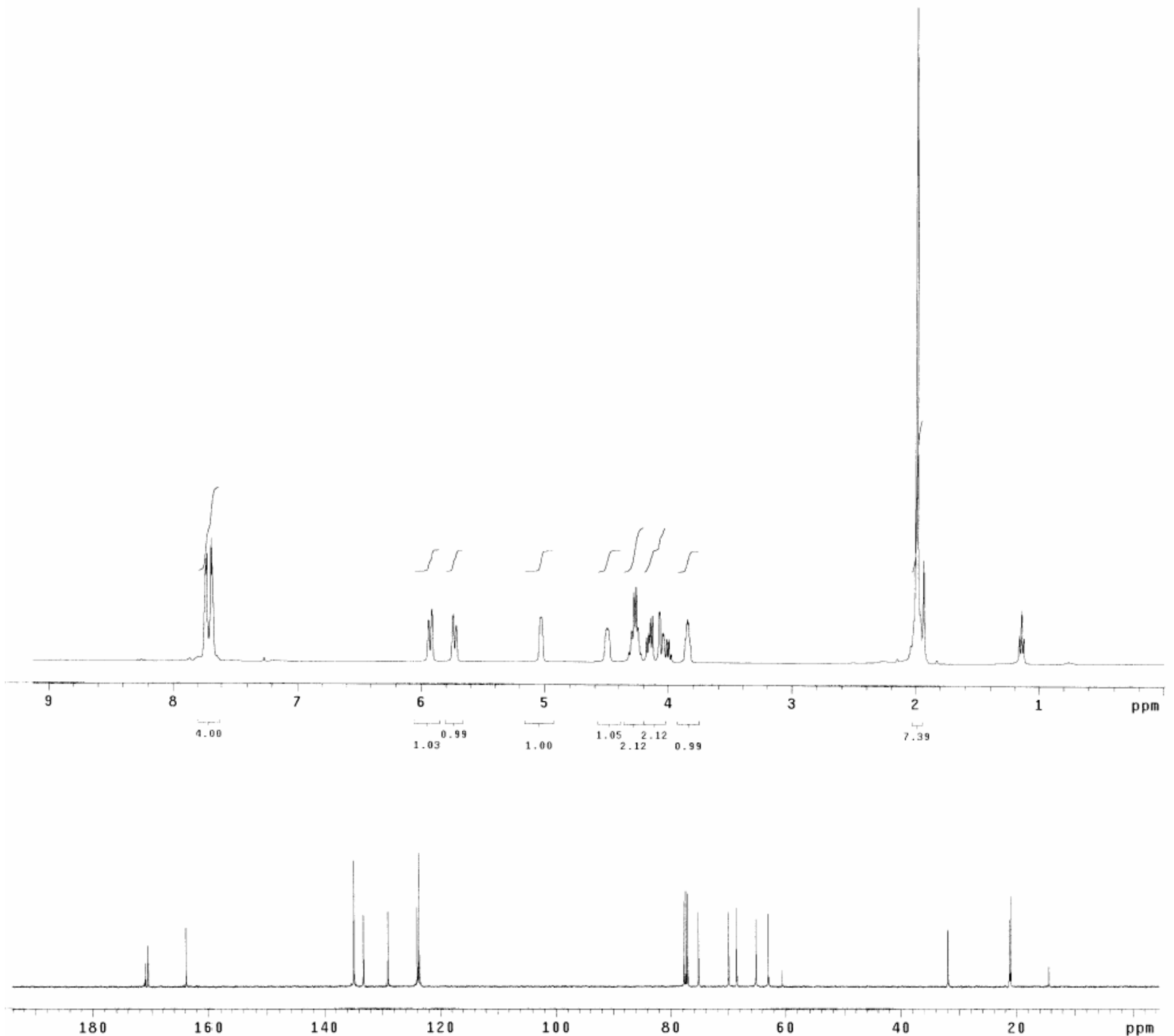


\section{Phth-12}
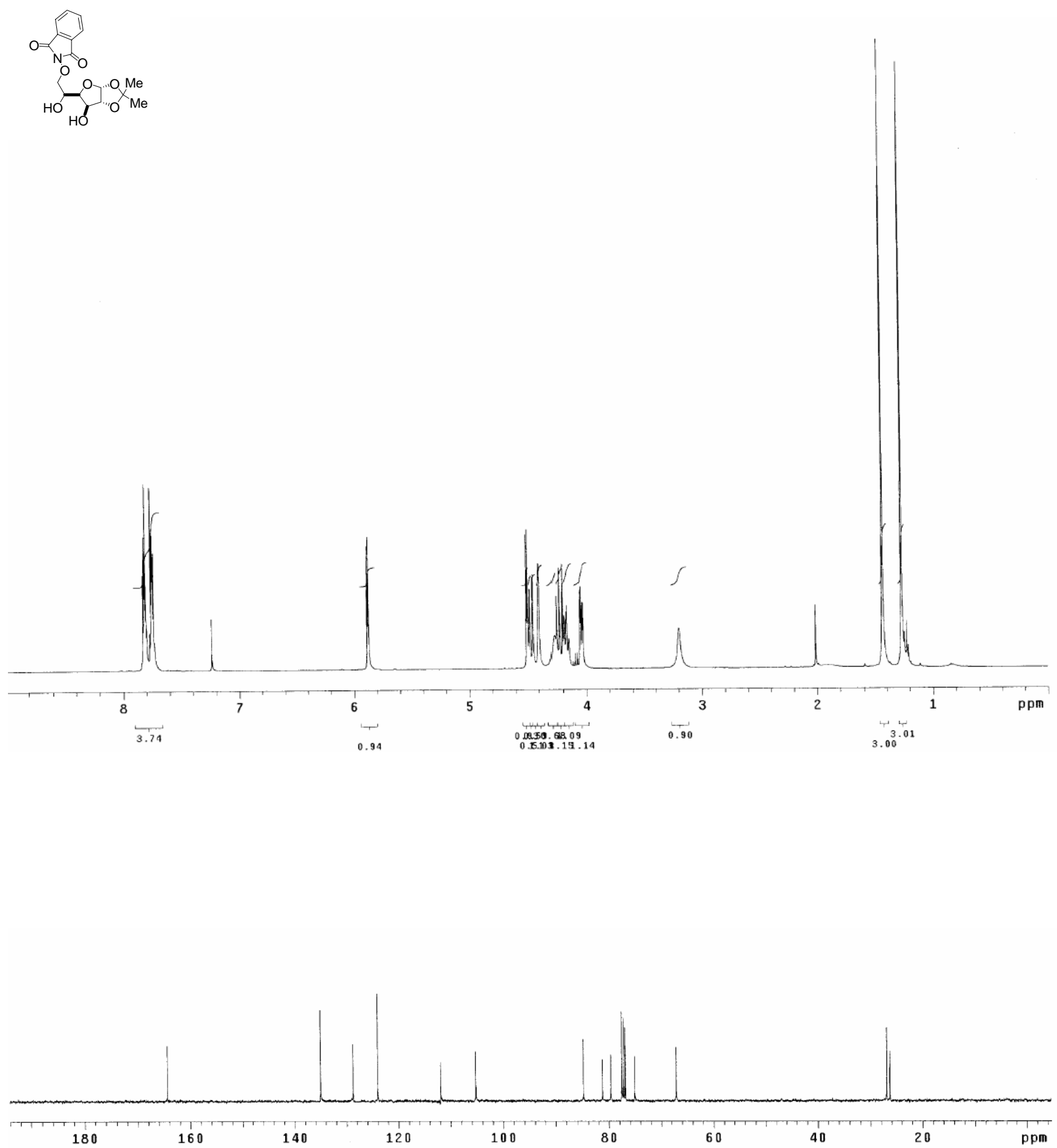
$\sum^{O} i^{O H}$
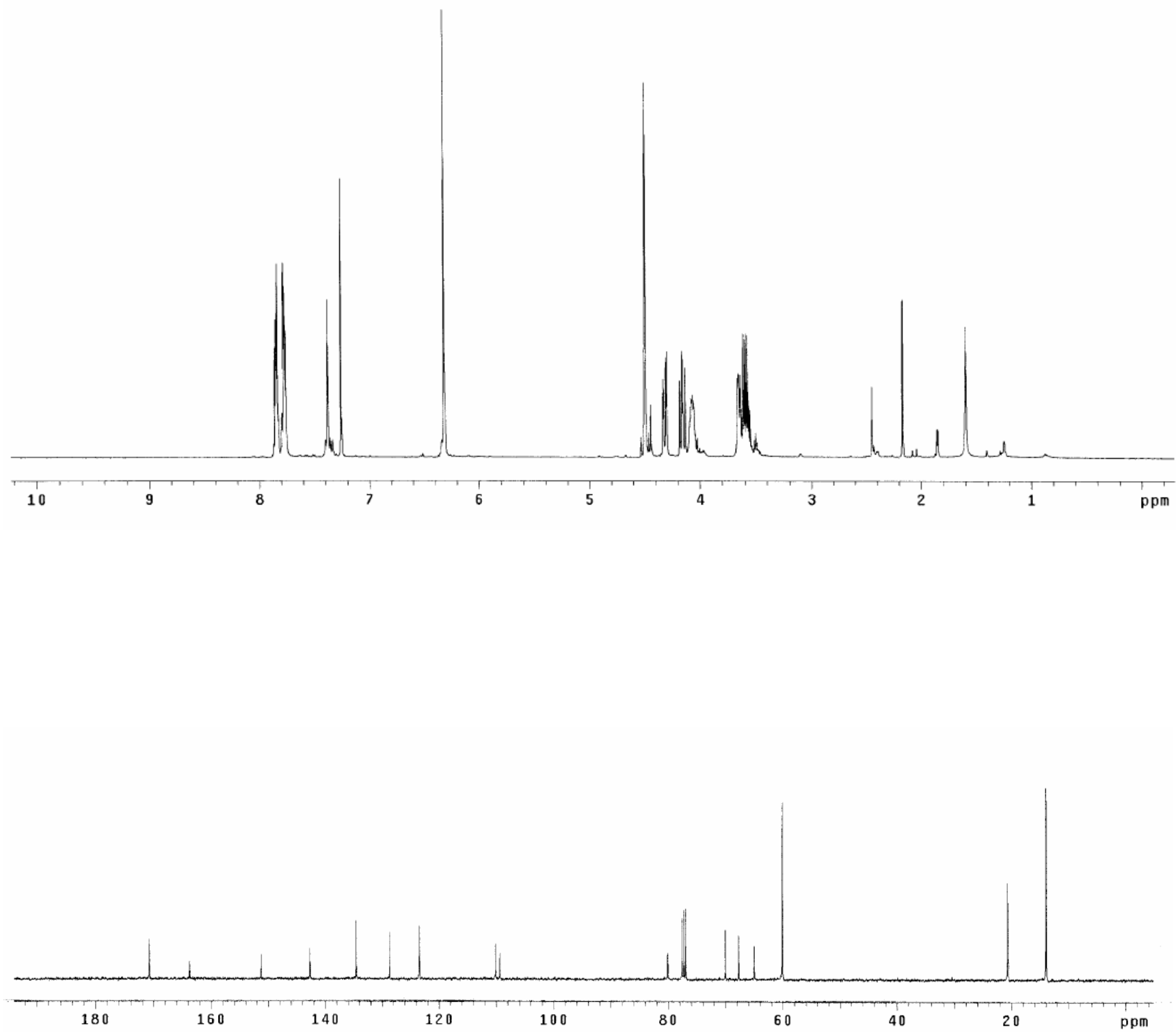


\section{Oxime 45}
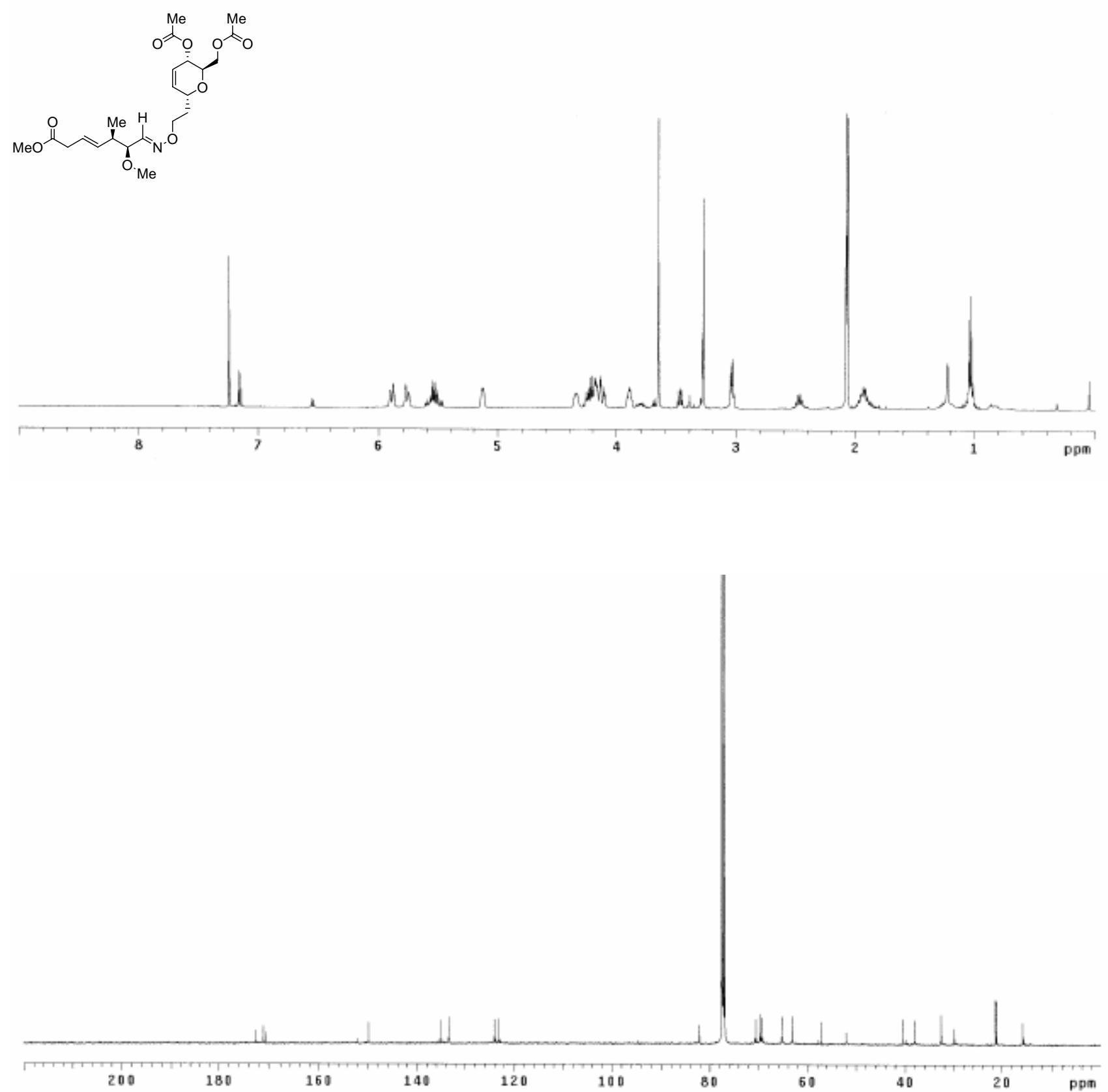


\section{Oxime 46}
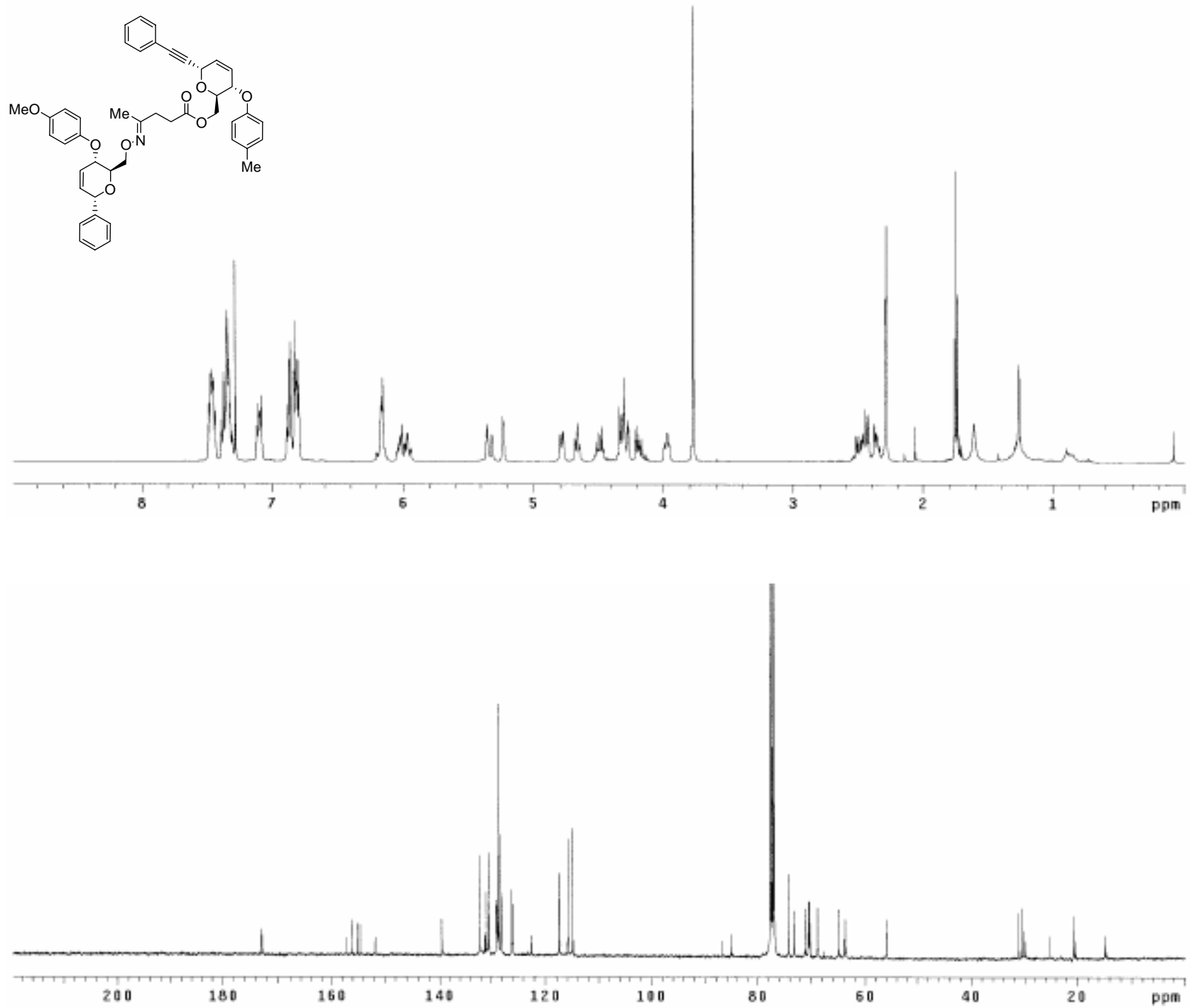


\section{Oxime 47}
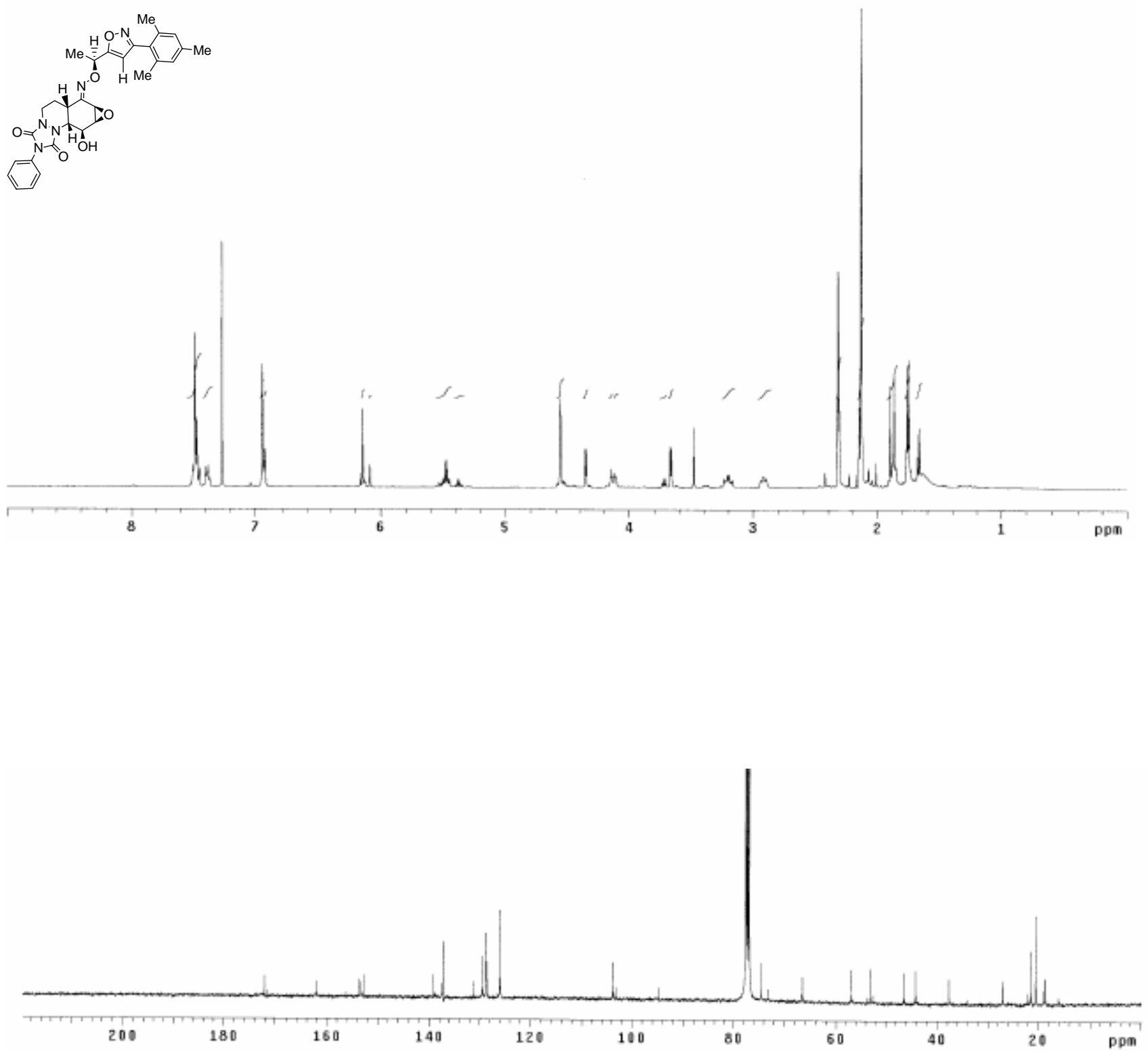


\section{Oxime 48}
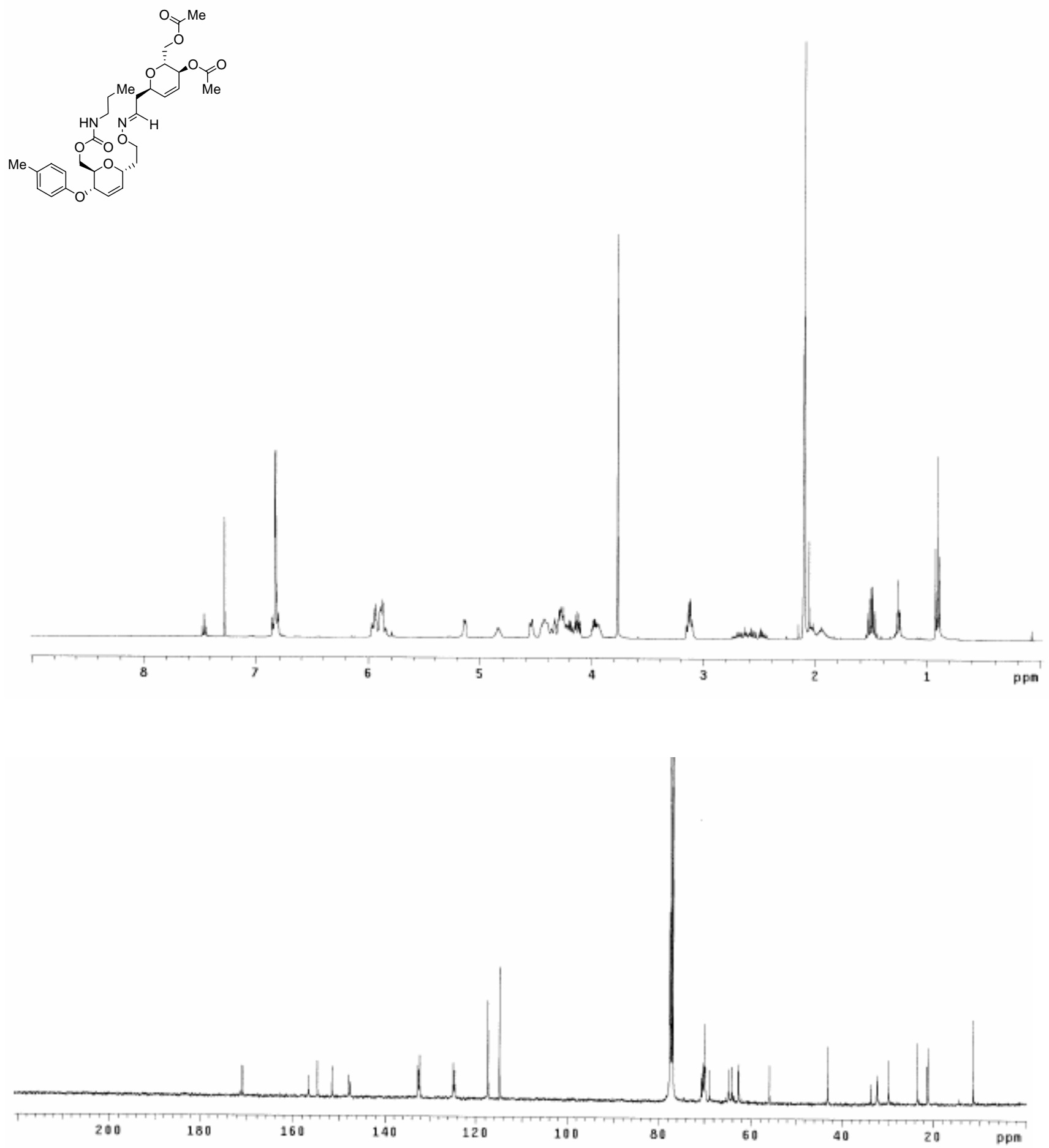

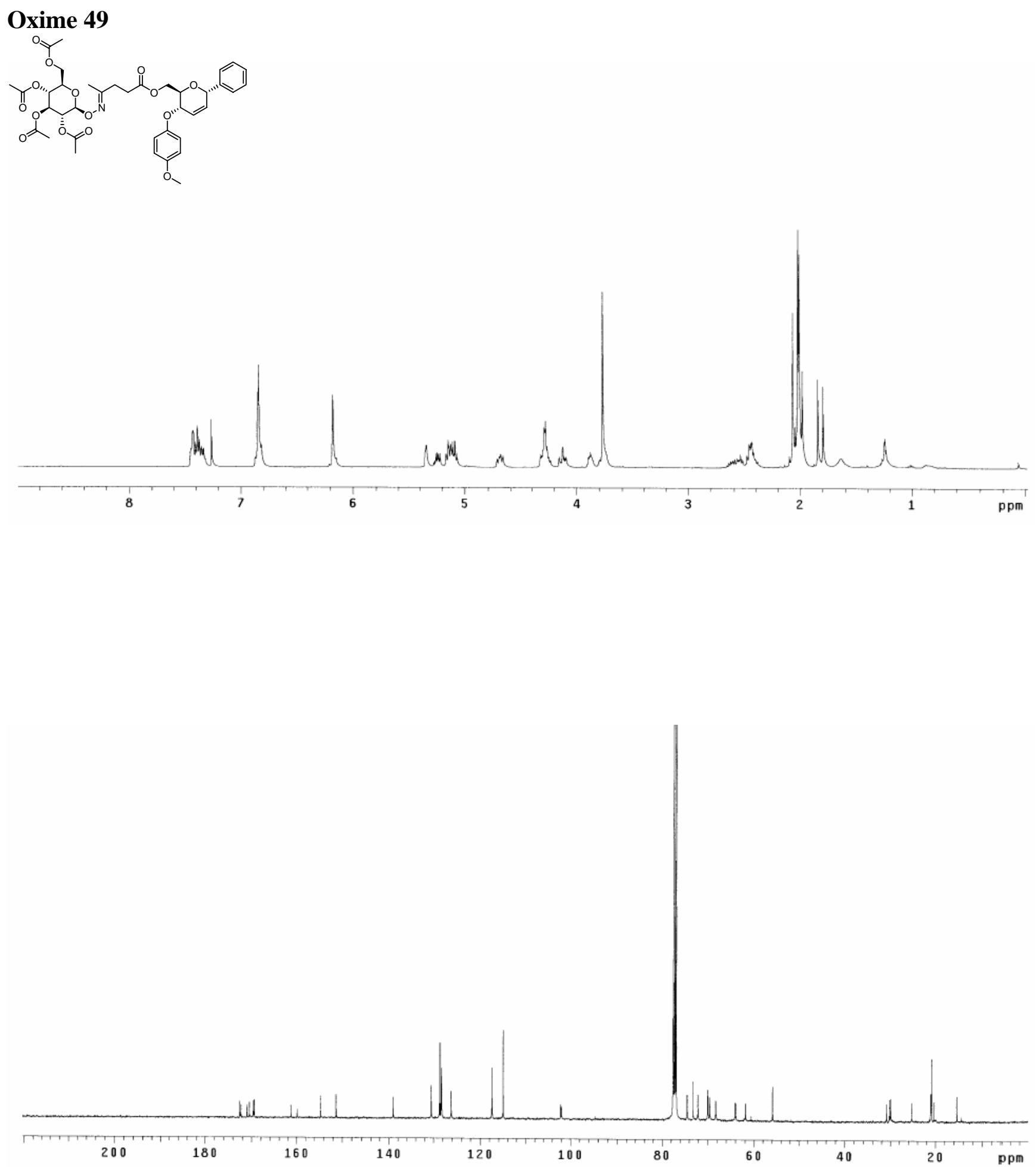

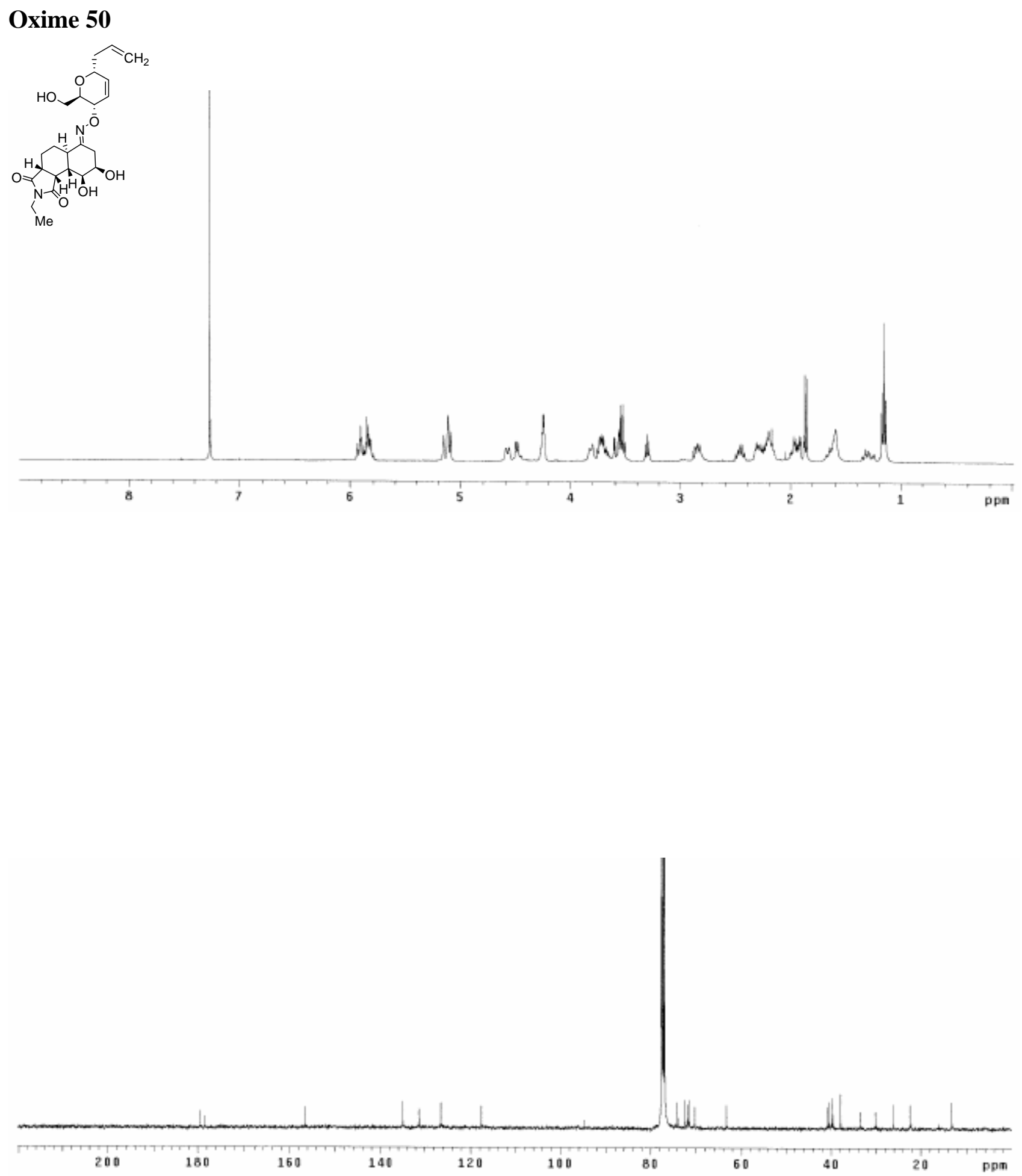


\section{Oxime 51}
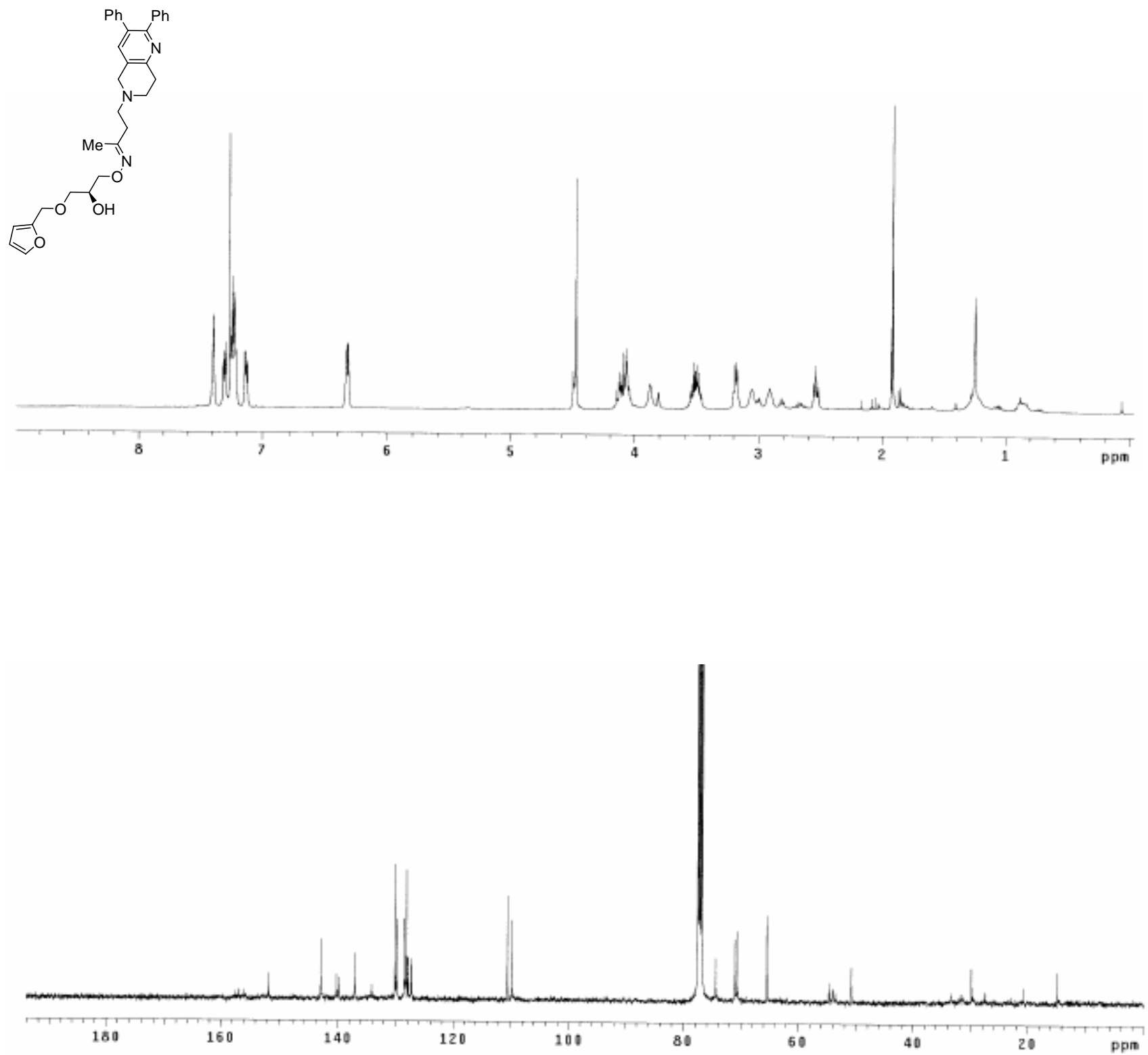

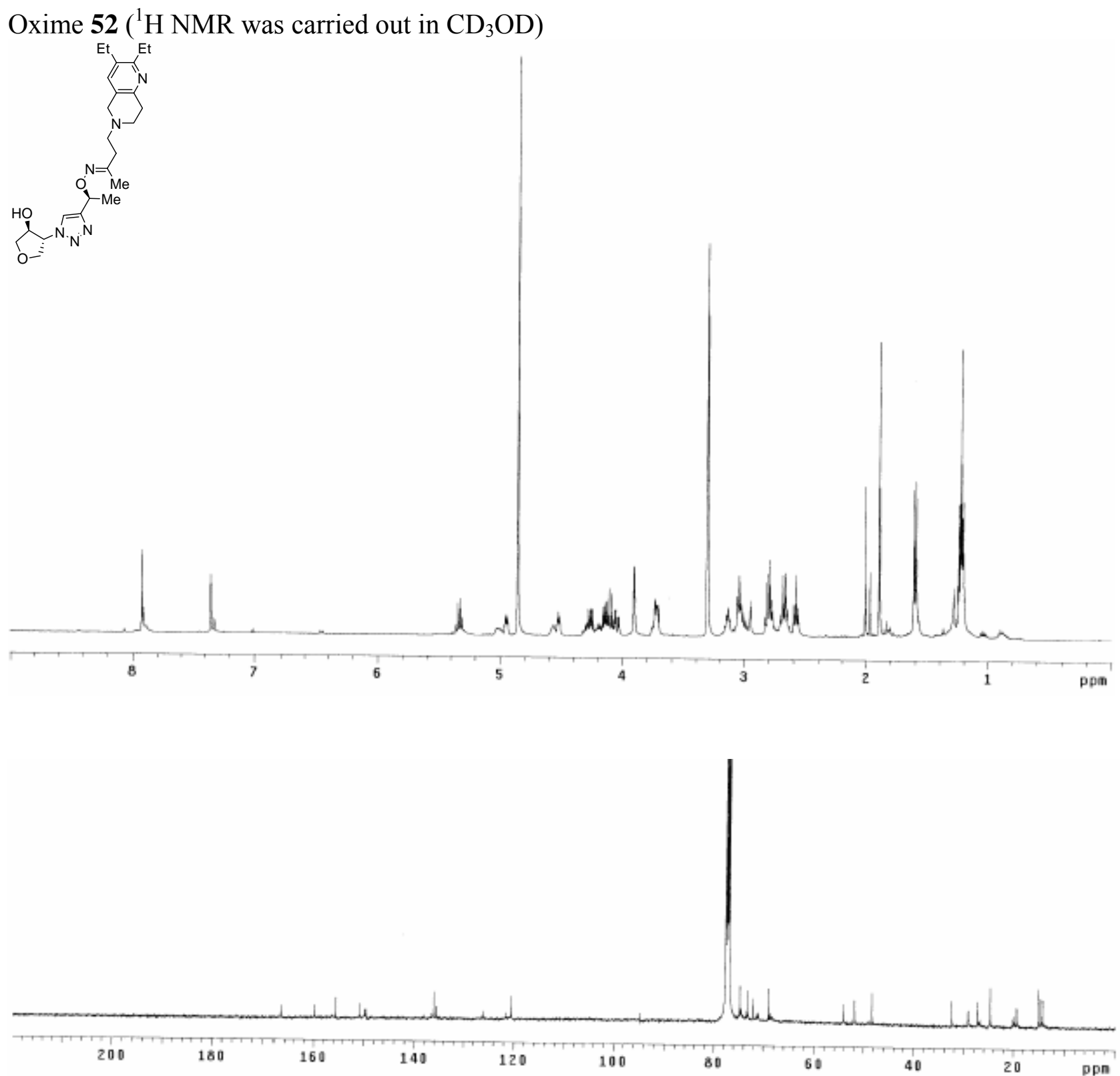
Oxime $53\left({ }^{1} \mathrm{H}\right.$ NMR was carried out in $\left.\mathrm{CD}_{3} \mathrm{OD}\right)$
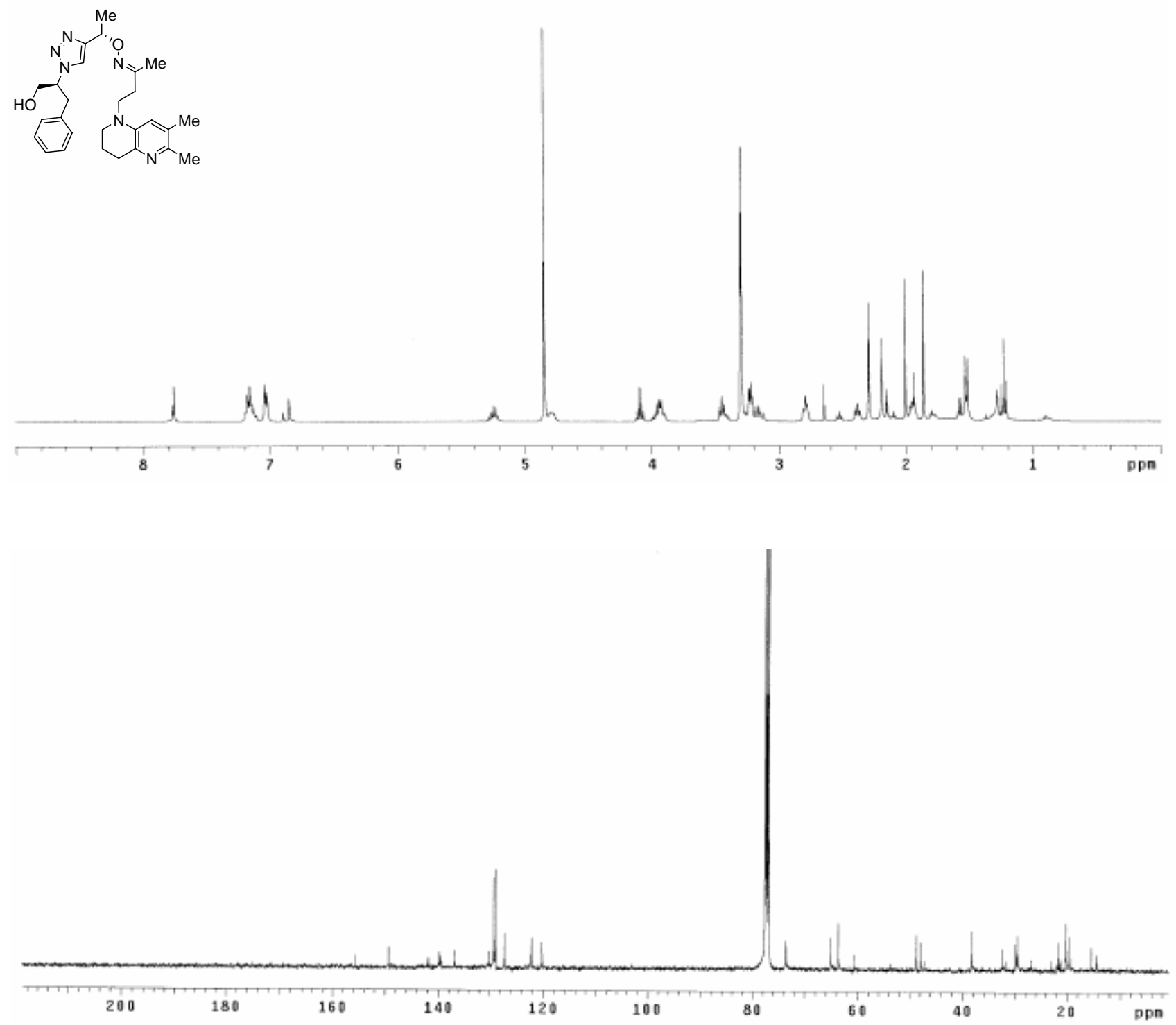


\section{Oxime 54}
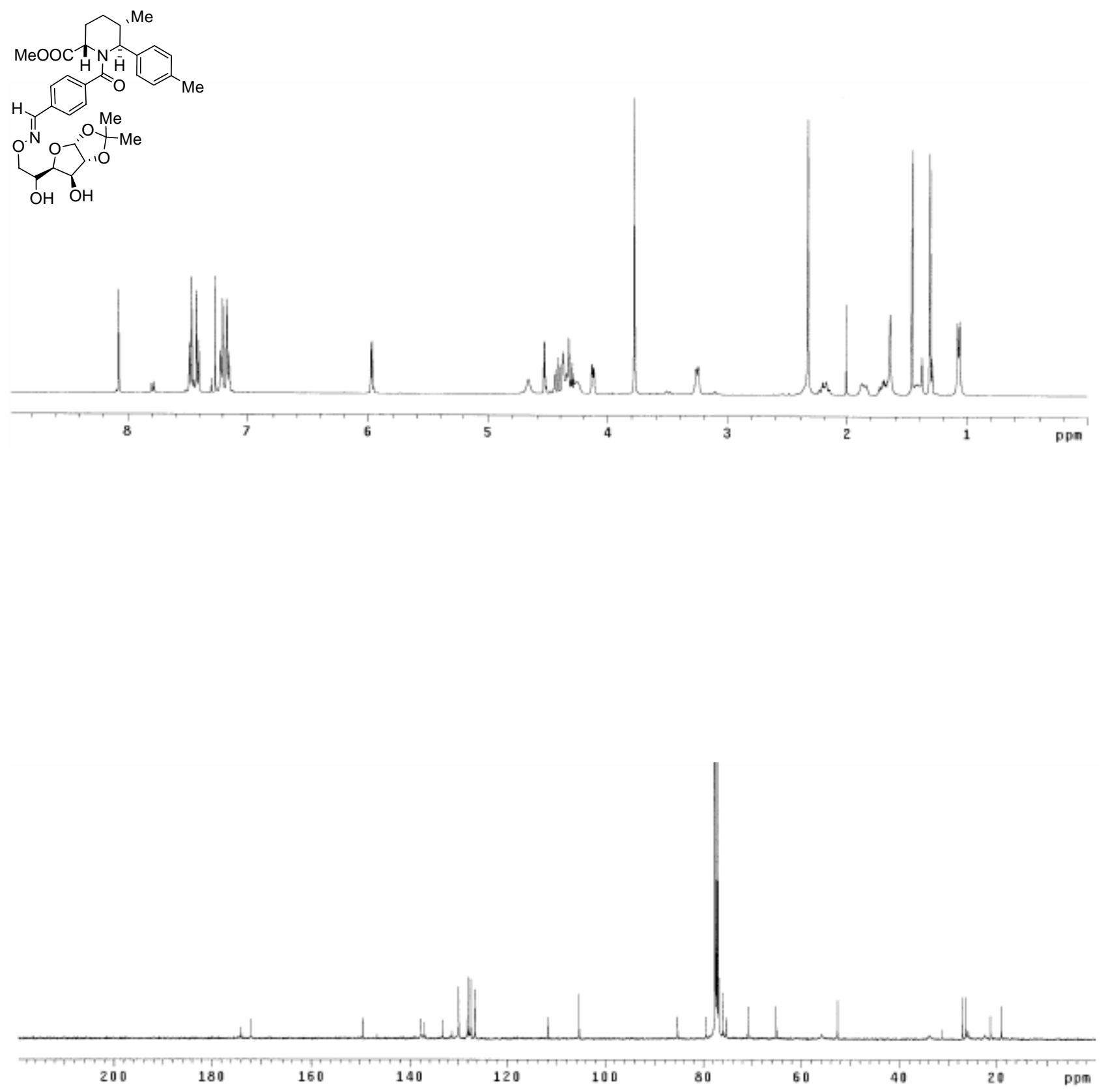


\section{Oxime 55}
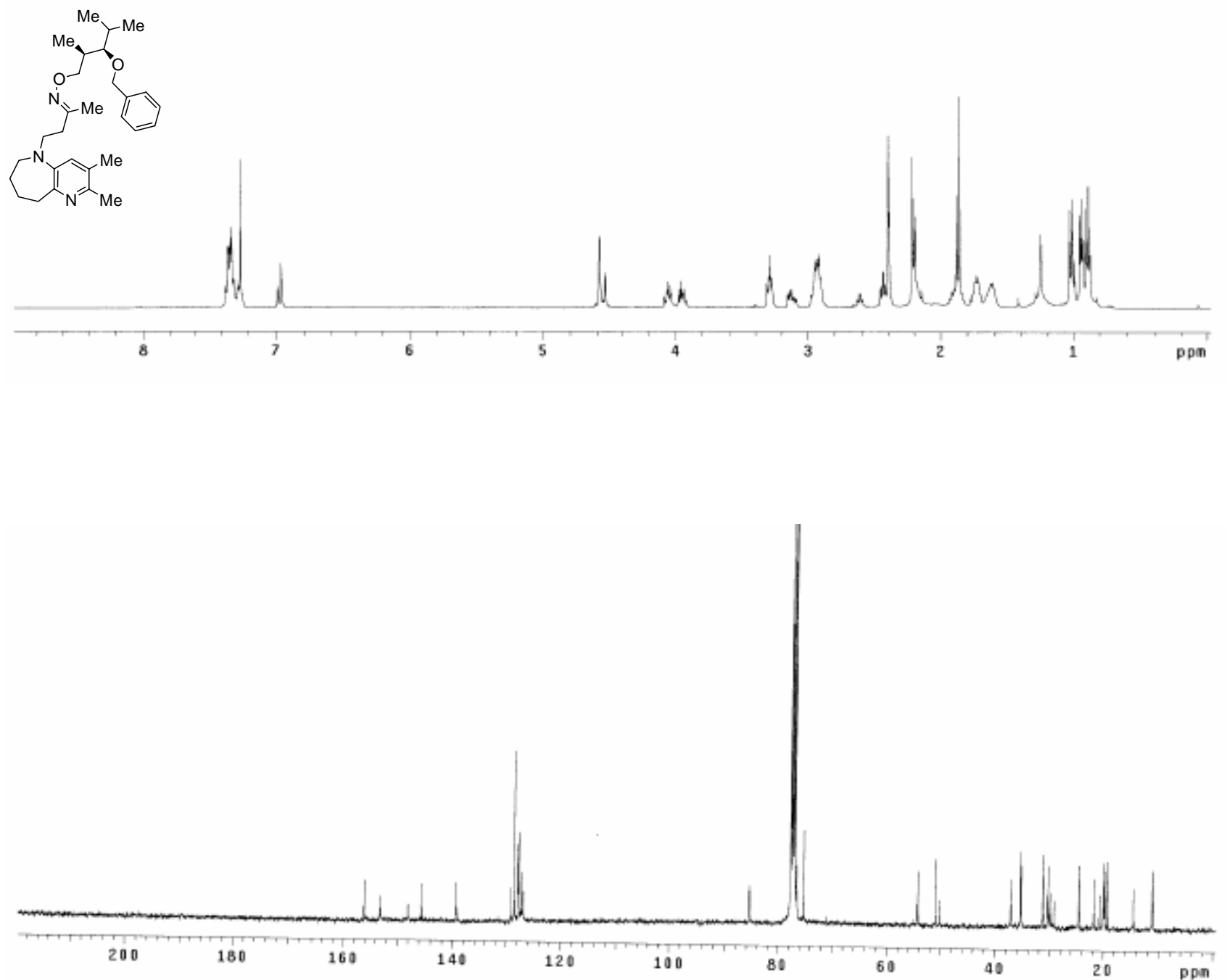


\section{Oxime 56}
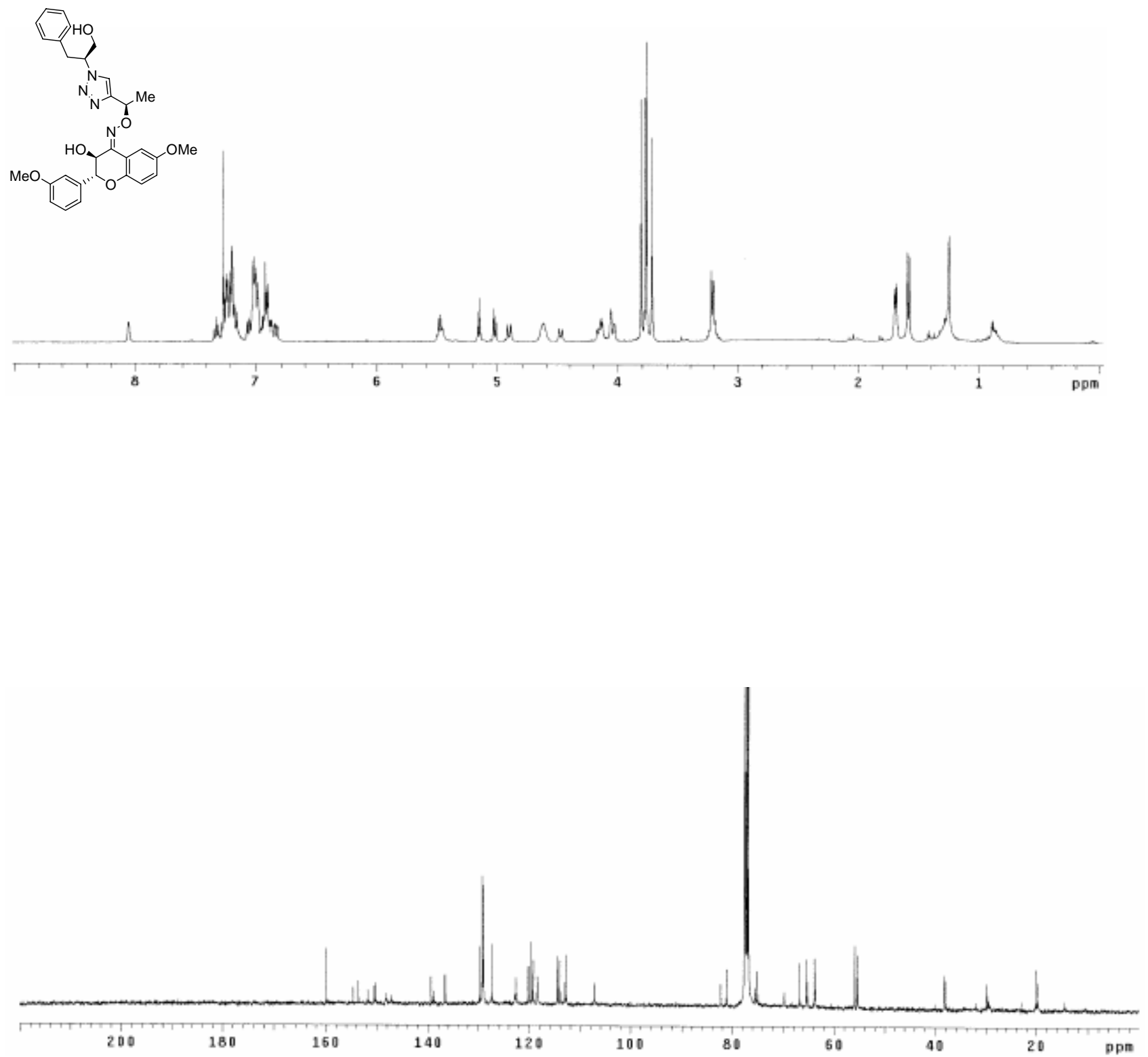
Openlynx Report -

Sample: 915

File:SS-Lib-B14A

Time:17:37:24

Printed: Fri Nov 05 11:14:53 2004

Sample Report (continued):

Sample 915 Vial 5,3:3 ID File SS-Lib-B14A3 Date 29-Oct-2004 Time 17:37:24 Description

ELSD Smooth (SG, 2x2)

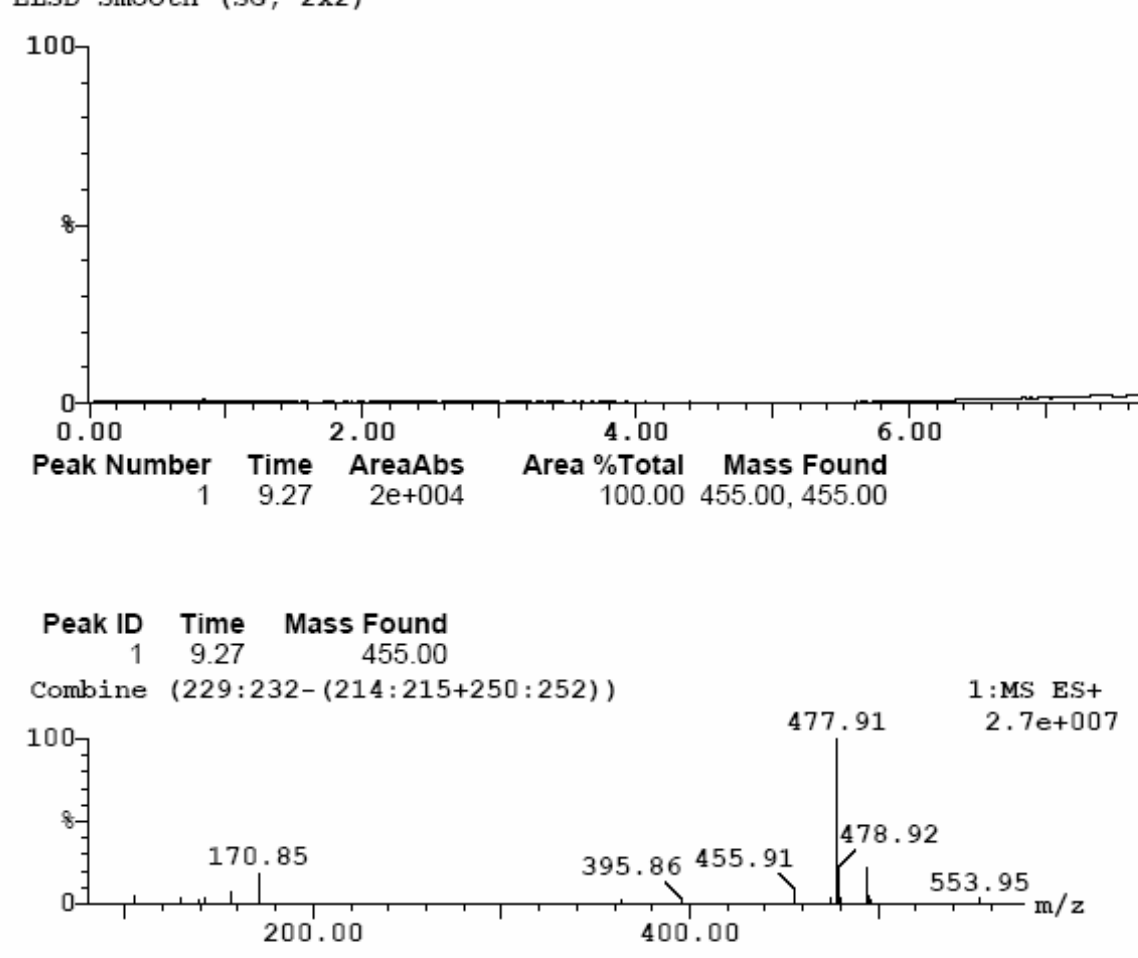

$1.4 e+005$
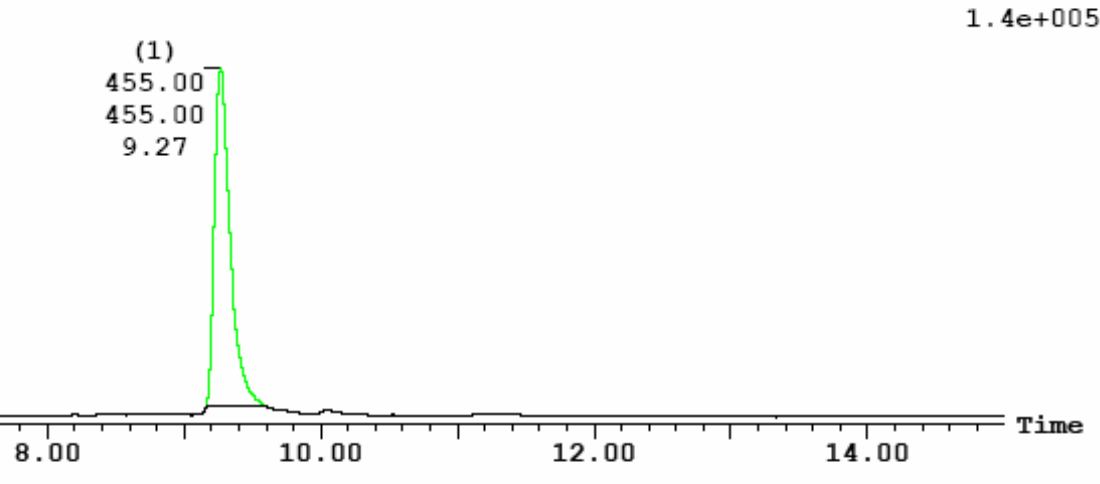

10.00

14.00

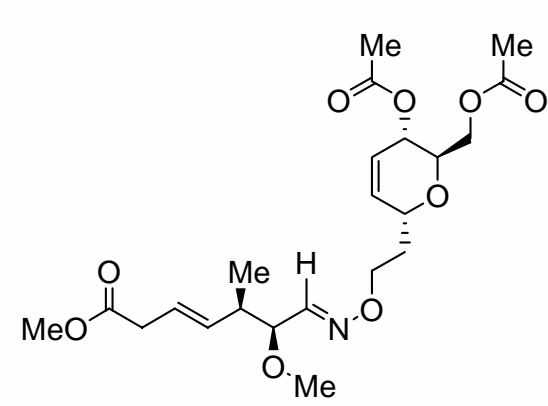




\begin{tabular}{lll}
\hline Openlynx Report - & Page 2 \\
Sample: 733 & Vial:5,3:15 & ID: \\
File:SS-Lib-B2A1 & Date:20-Oct-2004 & Time:04:35:28 \\
Description: & & \\
Printed: Thu Nov 04 21:37:19 2004 & &
\end{tabular}

Sample Report:

Sample 733 Vial 5,3:15 ID File SS-Lib-B2A1 Date 20-Oct-2004 Time 04:35:28 Description

ELSD Smooth (SG, 2x2)

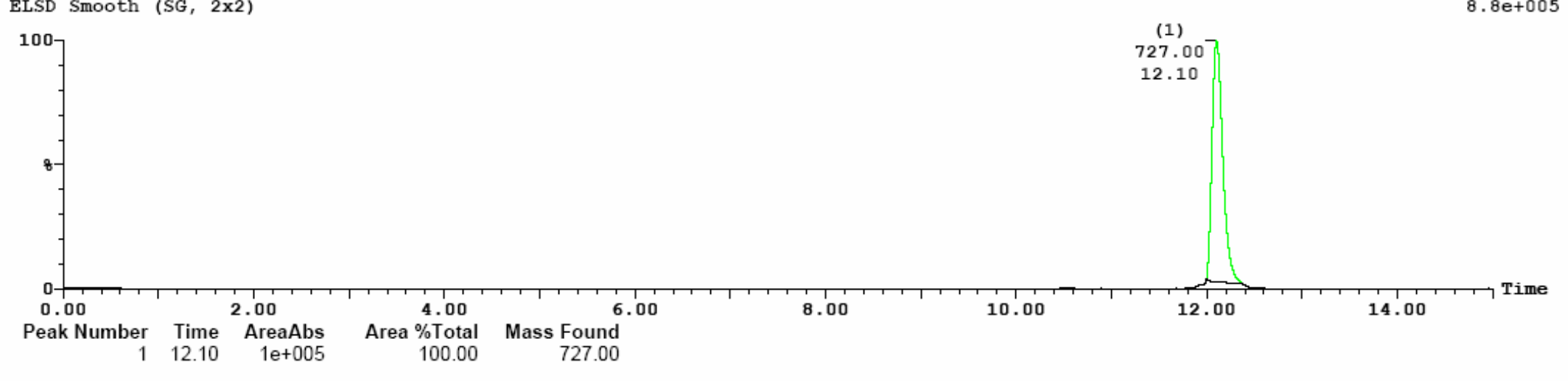

\section{Peak ID Time Mass Found}

Combine $(299: 302-(284: 285+320: 321))$

$1:$ MS ES+
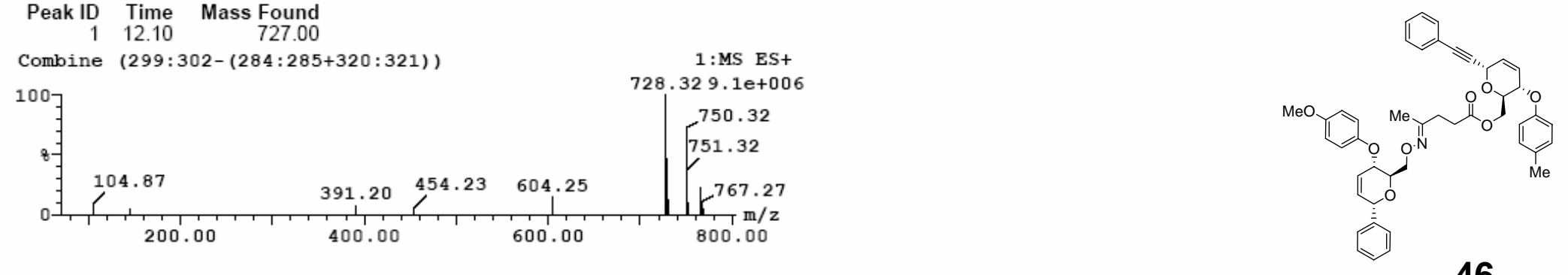

SP-2 


\begin{tabular}{lll}
\hline Openlynx Report - & & Page 1 \\
Sample: 867 & Vial:5, $1: 25$ & ID: \\
File:SS-Lib-B10A11 & Date:30-Oct-2004 & Time:06:39:58 \\
Description: & & \\
Printed: Sat Jan 29 16:17:12 2005 & & \\
\hline
\end{tabular}

Sample Report:

Sample 867 Vial 5,1:25 ID File SS-Lib-B10A11 Date 30-Oct-2004 Time 06:39:58 Description BLSD Smooth (SG, 3×3)

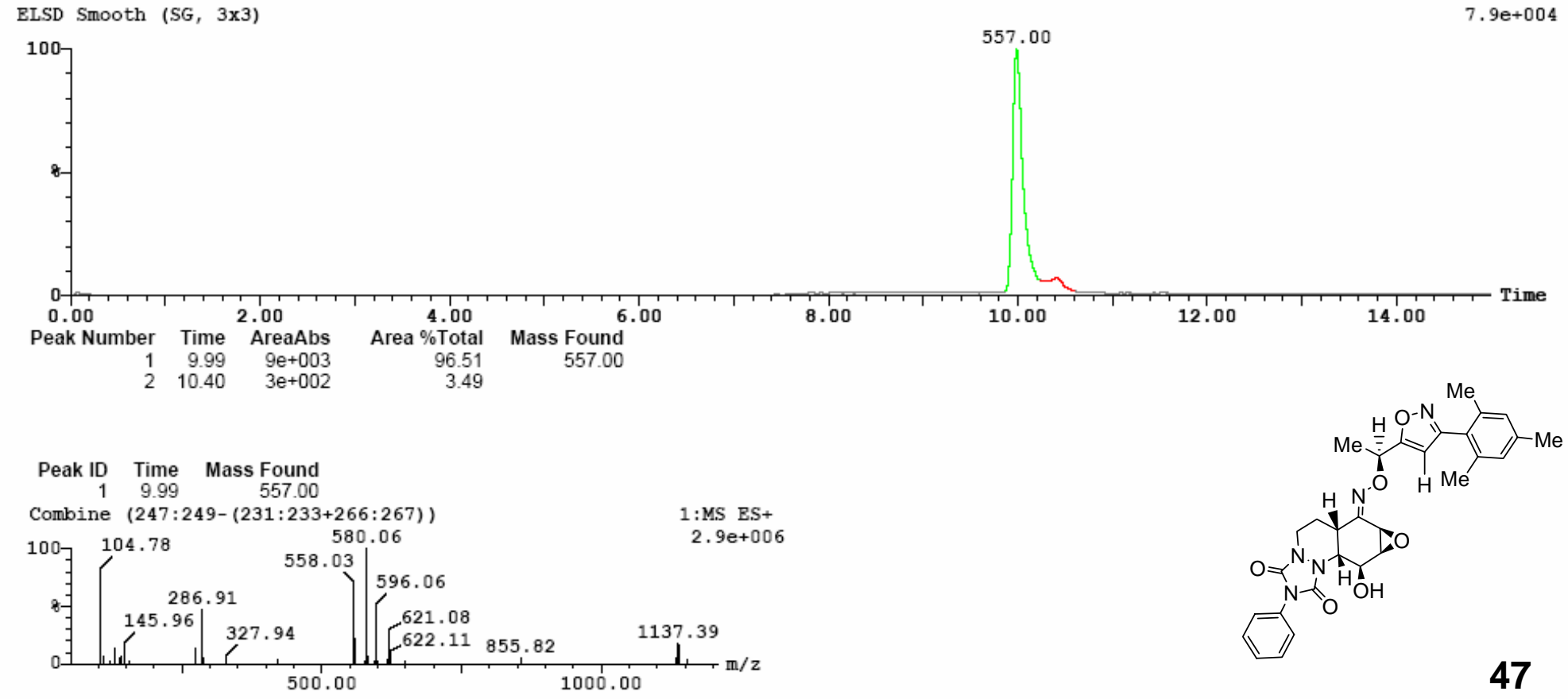




\begin{tabular}{lll}
\hline Openlynx Report - & & Page 3 \\
Sample: 749 & Vial:5,3:30 & ID: \\
File:SS-Lib-B3A2 & Date:20-Oct-2004 & Time:09:21:09 \\
Description: & & \\
Printed: Thu Nov 04 21:45:04 2004 & &
\end{tabular}

Printed: Thu Nov 04 21:45:04 2004

Sample Report (continued):

Sample 749 Vial 5,3:30 ID File SS-Lib-B3A2 Date 20-Oct-2004 Time 09:21:09 Description

ELSD Smooth (SG，2x2)

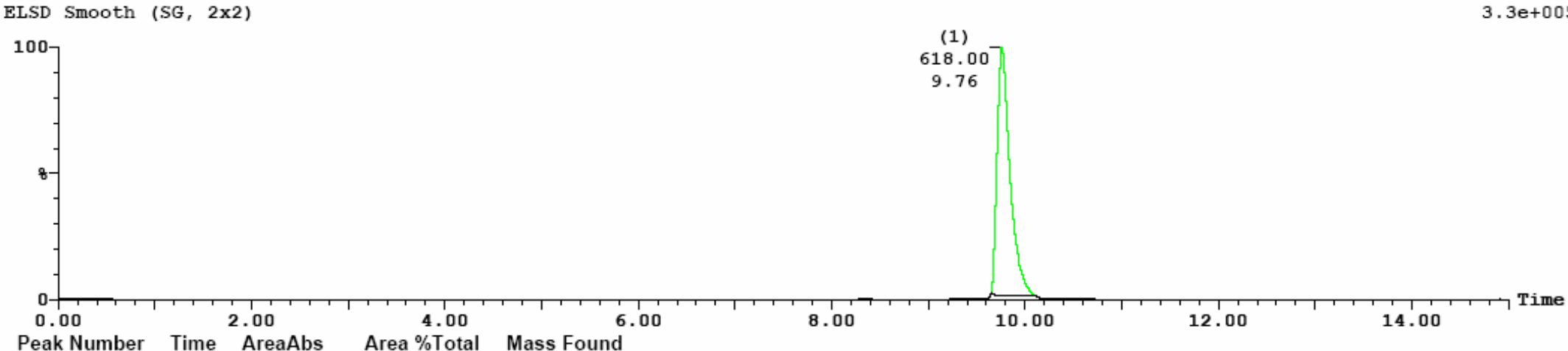
Peak Number Time $\begin{array}{rr}\text { AreaAbs } \\ 5 \mathrm{e}+004\end{array}$

Peak ID Time Mass Found
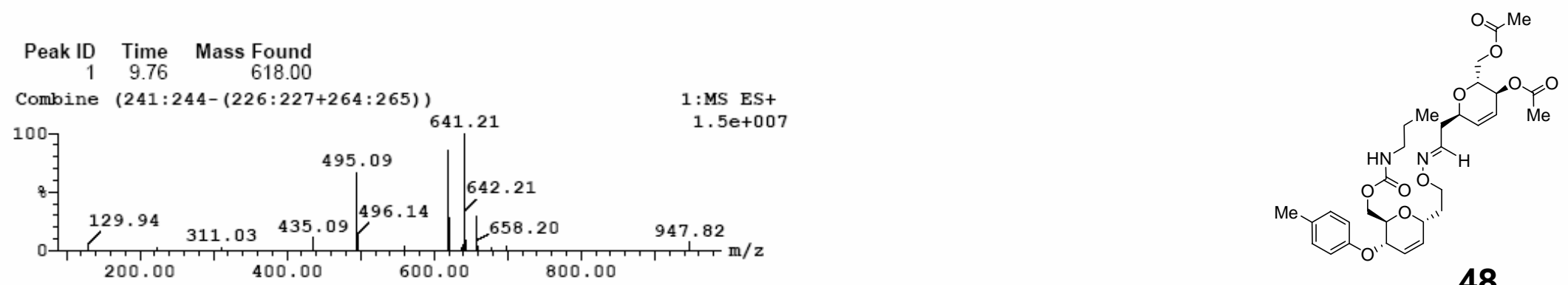


\begin{tabular}{lll}
\hline Openlynx Report - & & Page 14 \\
Sample: 730 & Vial:5,3:13 & ID: \\
File:SS-Lib-B1A13 & Date:20-Oct-2004 & Time:03:54:29 \\
Description: & & \\
Printed: Thu Nov 04 21:33:16 2004 & &
\end{tabular}

Printed: Thu Nov 04 21:33:16 2004

Sample Report (continued):

Sample 730 Vial 5,3:13 ID File SS-Lib-B1A13 Date 20-Oct-2004 Time 03:54:29 Description

ELSD Smooth (SG，2×2)

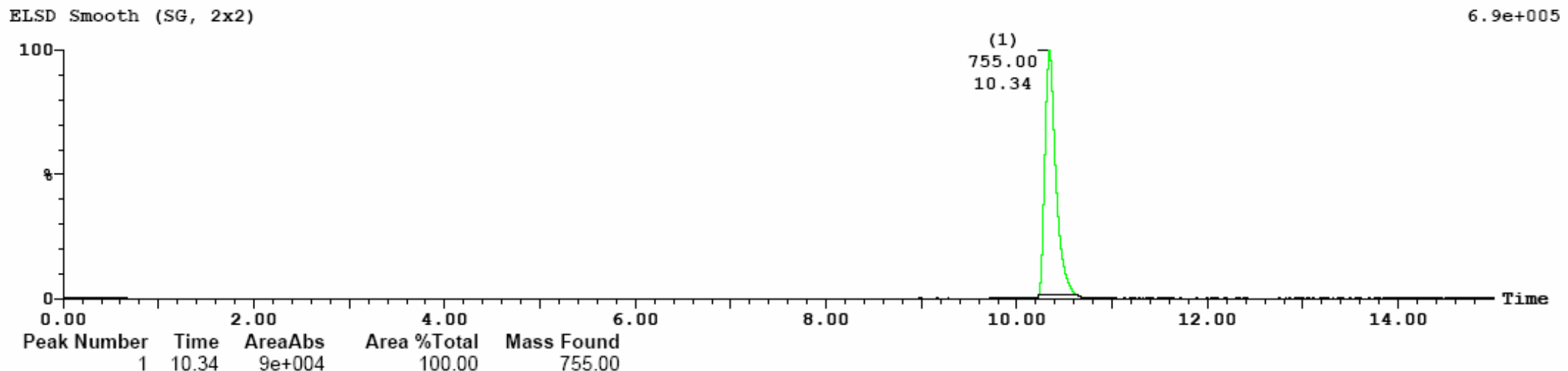

Peak Number Time AreaAbs Area \%Total Mass Found

\section{Peak ID Time Mass Found}

Combine $(256: 258-(241: 242+276: 278))$
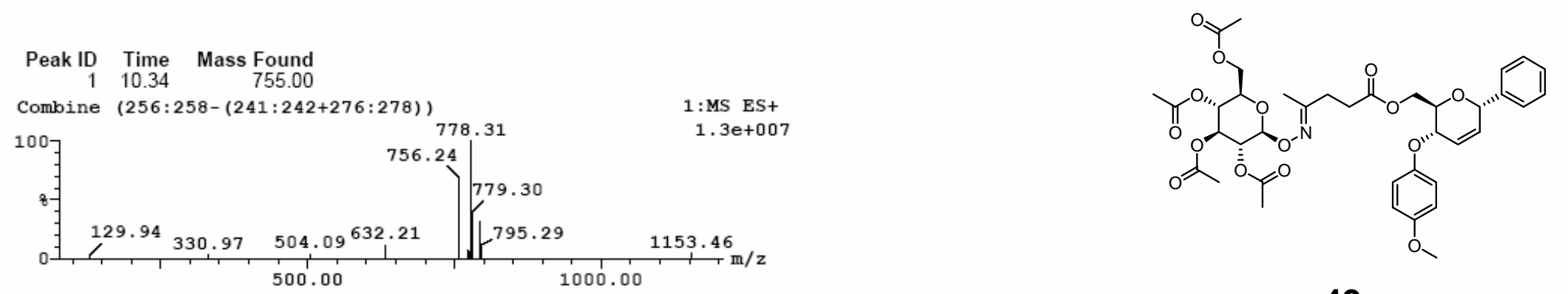


\begin{tabular}{lll}
\hline Openlynx Report - & & Page 8 \\
Sample: 844 & Vial:5,3:10 & ID: \\
File:SSS-Lib-B9A7 & Date:01-Nov-2004 & Time:01:57:11 \\
Description: & & \\
Printed: Fri Nov 05 11:36:16 2004 & &
\end{tabular}

Printed: Fri Nov 05 11:36:16 2004

Sample Report (continued):

Sample 844 Vial 5,3:10 ID File SSS-Lib-B9A7 Date 01-Nov-2004 Time 01:57:11 Description

ELSD Smooth (SG, $2 \times 2)$

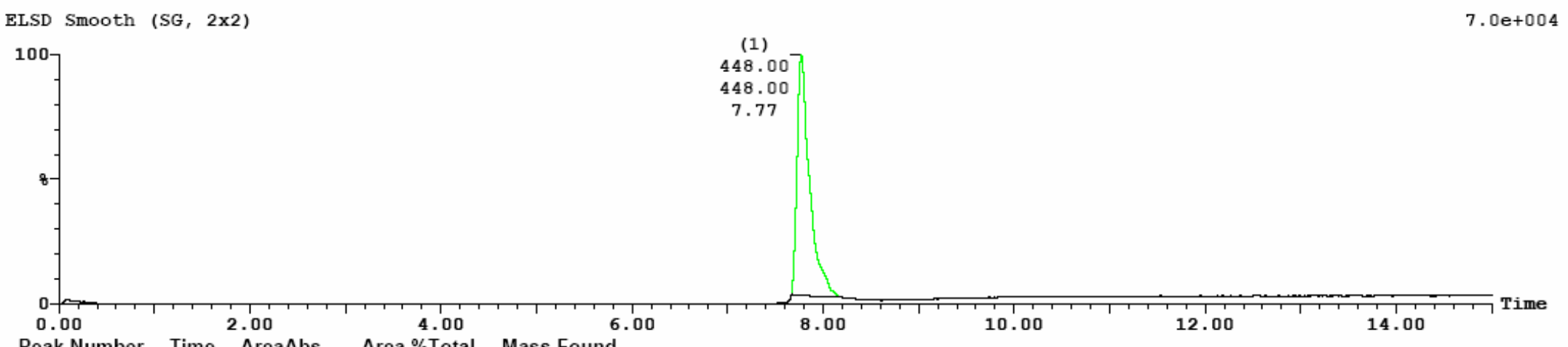

Peak Number Time AreaAbs Area \%Total Mass Found

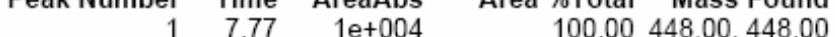

Peak ID Time Mass Found

Combine $(192: 194-(177: 178+215: 217)) \quad 1:$ MS ES+
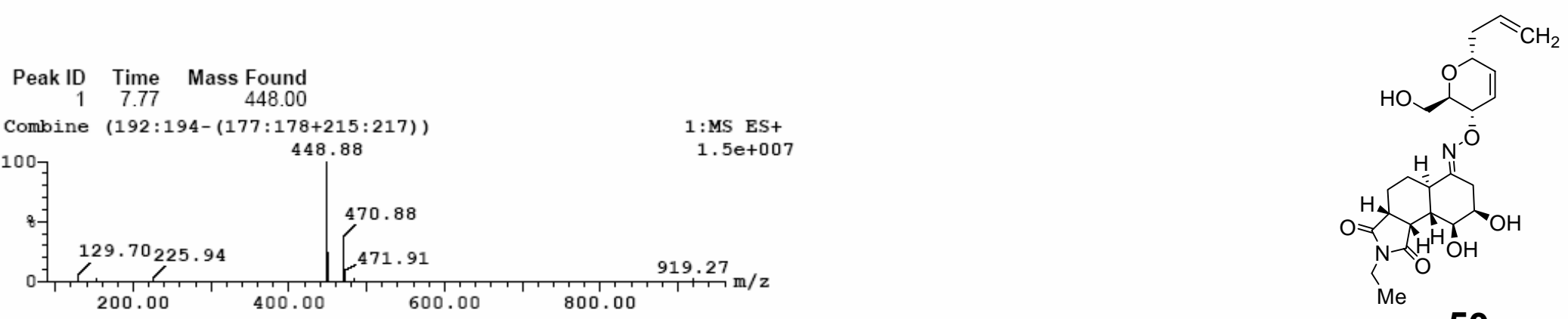

SP-6 
Description:

Vial:5,3:8

Date:31-Oct-2004

Time:00:04:14

Printed: Fri Nov 05 11:20:08 2004

Sample Report (continued):

Sample 905 Vial 5,3:8 ID File SSS-Lib-B13A8 Date 31-Oct-2004 Time 00:04:14 Description

ELSD Smooth (SG，2x2)

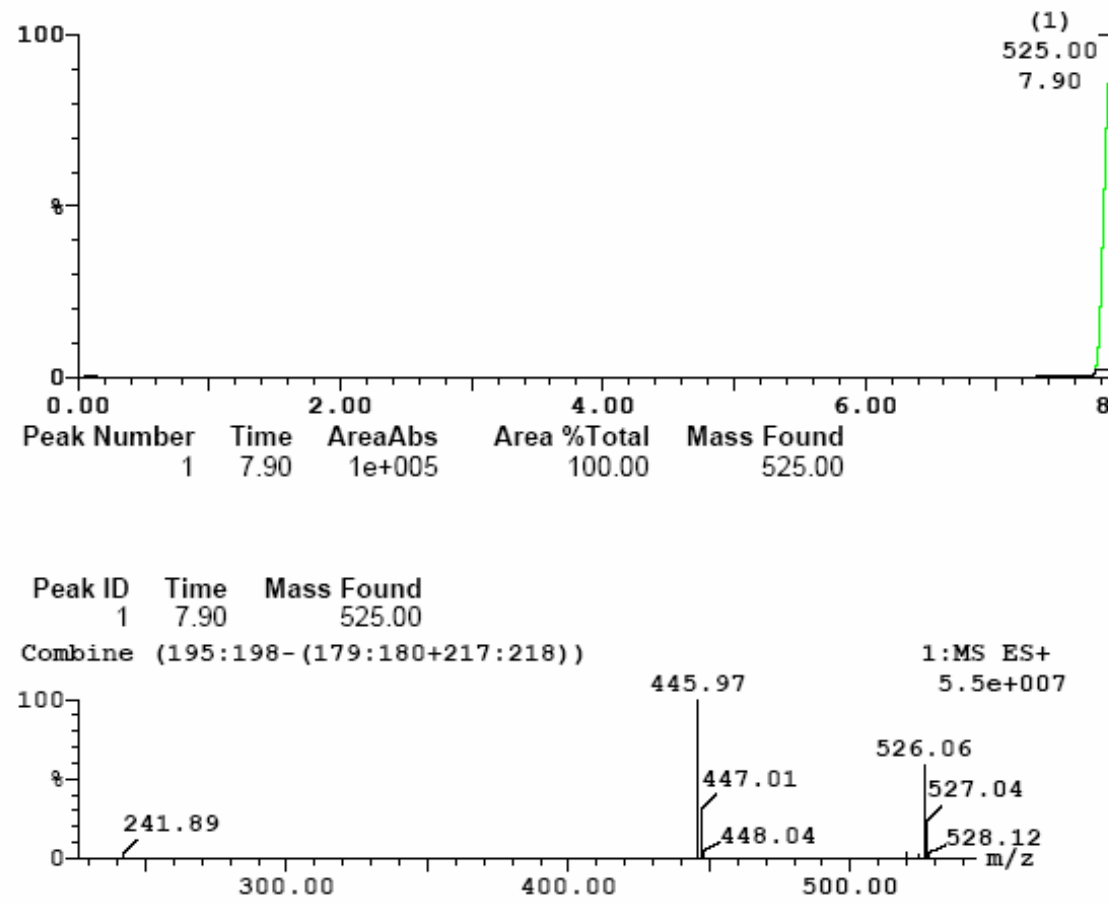

$6.2 e+005$

$$
\text { (1) }
$$$$
25.00
$$$$
90
$$

10.00

12.00

14.00

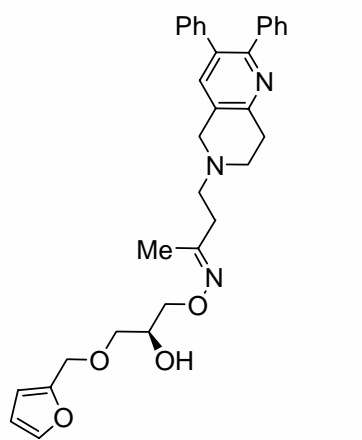


Openlynx Report -

Sample: 891

Vial:5,3:42

Date:01-Nov-2004

Time:22:58:00

Description:

Printed: Fri Nov 05 17:57:43 2004

Sample Report (continued):

Sample 891 Vial 5,3:42 ID File SSSS-Lib-B12A9 Date 01-Nov-2004 Time 22:58:00 Description

ELSD Smooth (SG，2x2)

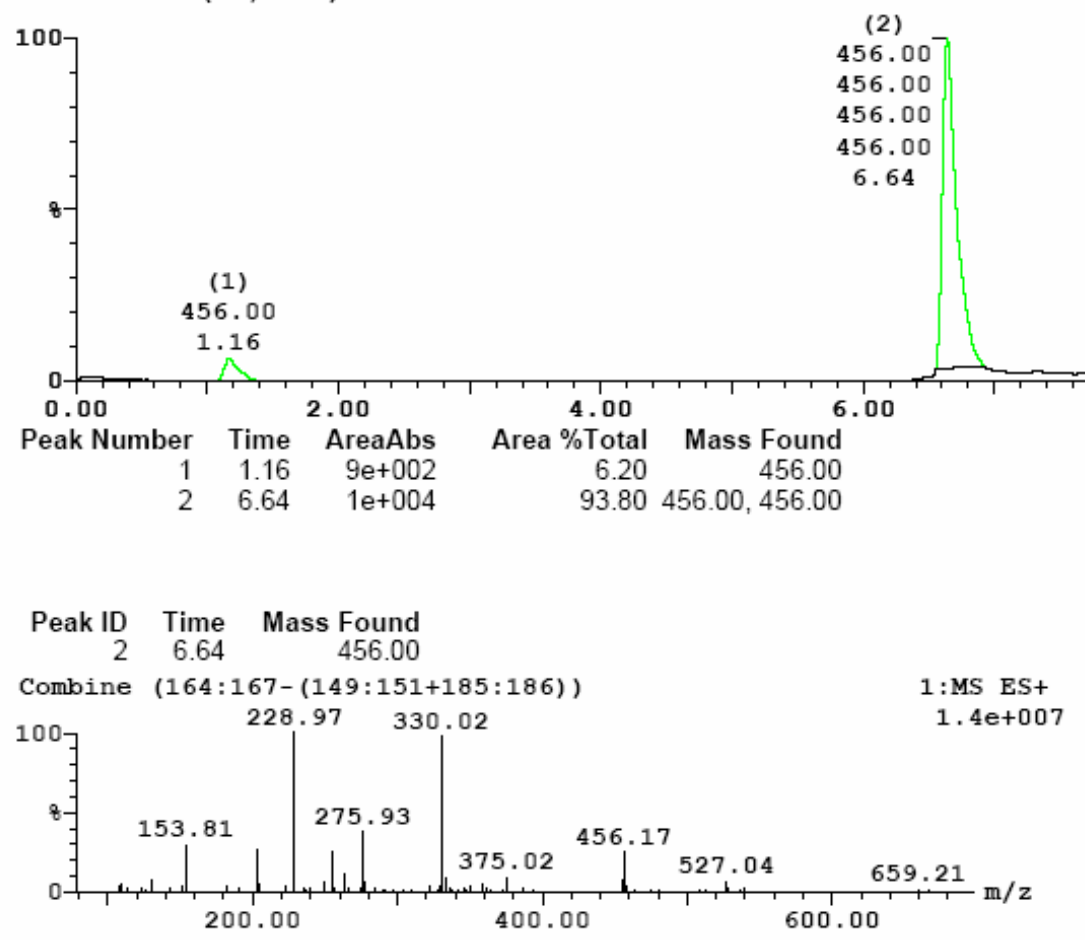

$1.1 e+005$

.00

10.00

12.00

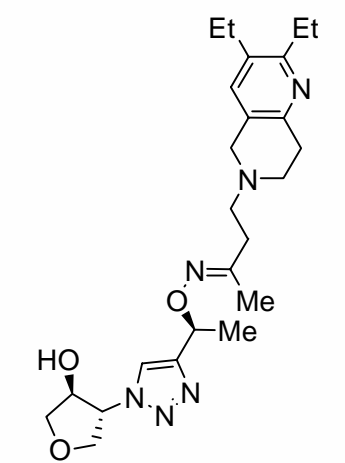

52

SP-8 


\begin{tabular}{lll}
\hline Openlynx Report - & & \\
Sample: 789 & Vial:5,3:34 & ID: \\
File:SSS-Lib-B5A10 & Date:31-Oct-2004 & Time:06:45:56 \\
Description: & & \\
Printed: Sat Jan 29 16:23:55 2005 & & \\
\hline
\end{tabular}

Sample Report:

Sample 789 Vial 5,3:34 ID File SSS-Lib-B5A10 Date 31-Oct-2004 Time 06:45:56 Description

BLSD Smooth (SG, 3×3)

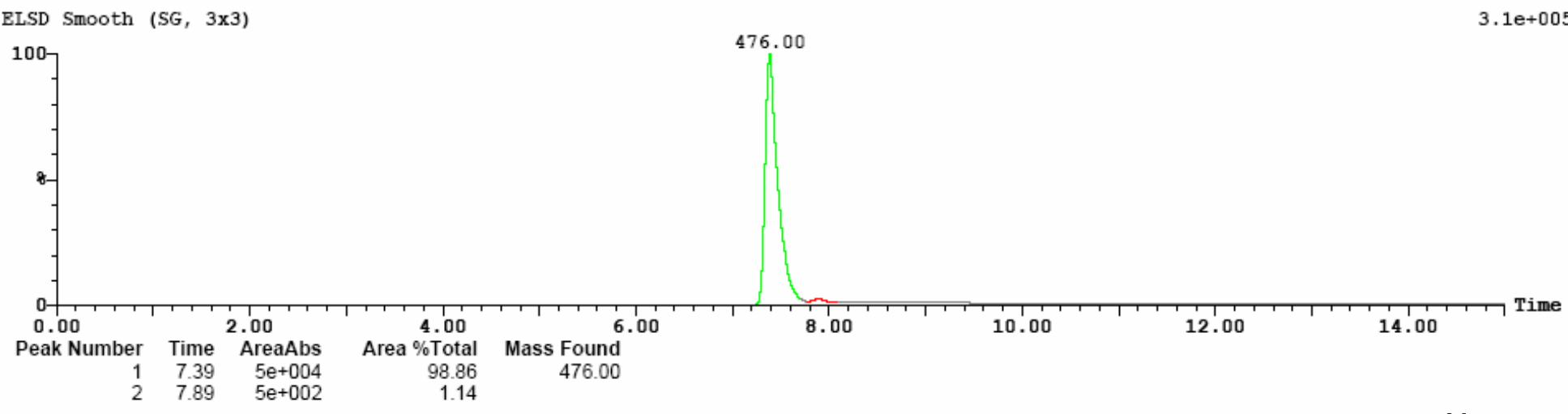

$3.1 \mathrm{e}+005$

$$
\begin{array}{rrr}
\text { Peak ID } & \text { Time } & \text { Mass Found } \\
1 & 7.39 & 476.00
\end{array}
$$

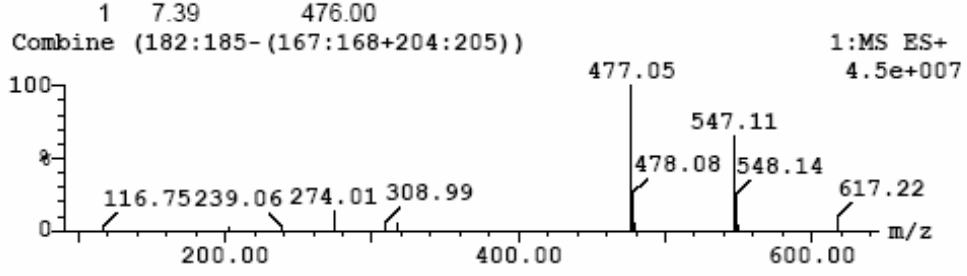

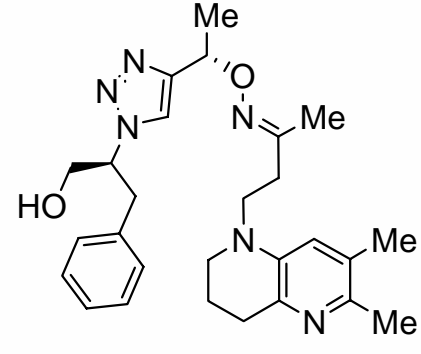

53 
Openlynx Report -

Sample: 798

File:SS-Lib-B6A6

Vial: $5,1: 6$

Date:24-Oct-2004

Description:

ID:

Printed: Thu Nov 04 22:28:55 2004

Sample Report (continued):

Sample 798 Vial 5,1:6 ID File SS-Lib-B6A6 Date 24-Oct-2004 Time 18:37:07 Description

ELSD Smooth (SG，2×2)

100

$6.0+005$

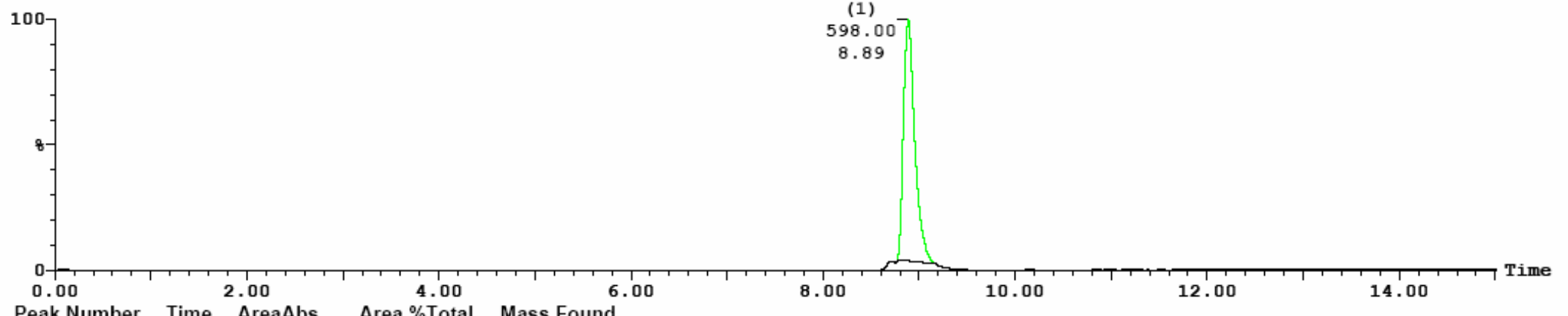

Peak Number Time AreaAbs Area \%Total Mass Found

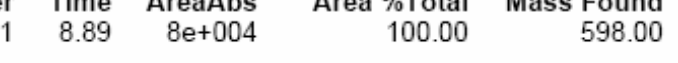

\section{$\begin{array}{rrr}\text { Peak ID } & \text { Time } & \text { Mass Found } \\ 1 & 8.89 & 598.00\end{array}$}

$\begin{array}{ccc}1 & 8.89 & 598.00 \\ \text { Combine } & (220: 222-(204: 206+240: 241))\end{array}$
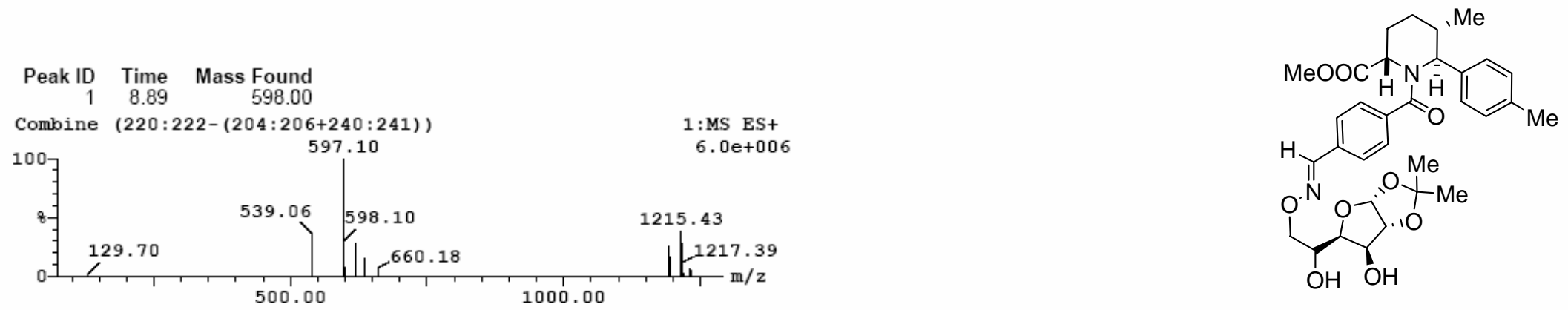
Openlynx Report -

Sample: 774

Vial: $5,1: 12$

Date:23-Oct-2004

Fescription:

Printed: Fri Nov 05 11:21:54 2004

Sample Report (continued):

Sample 774 Vial 5,1:12 ID File SS-Lib-B4A12 Date 23-Oct-2004 Time 22:24:06 Description

ELSD Smooth $(\mathrm{SG}, 2 \times 2)$

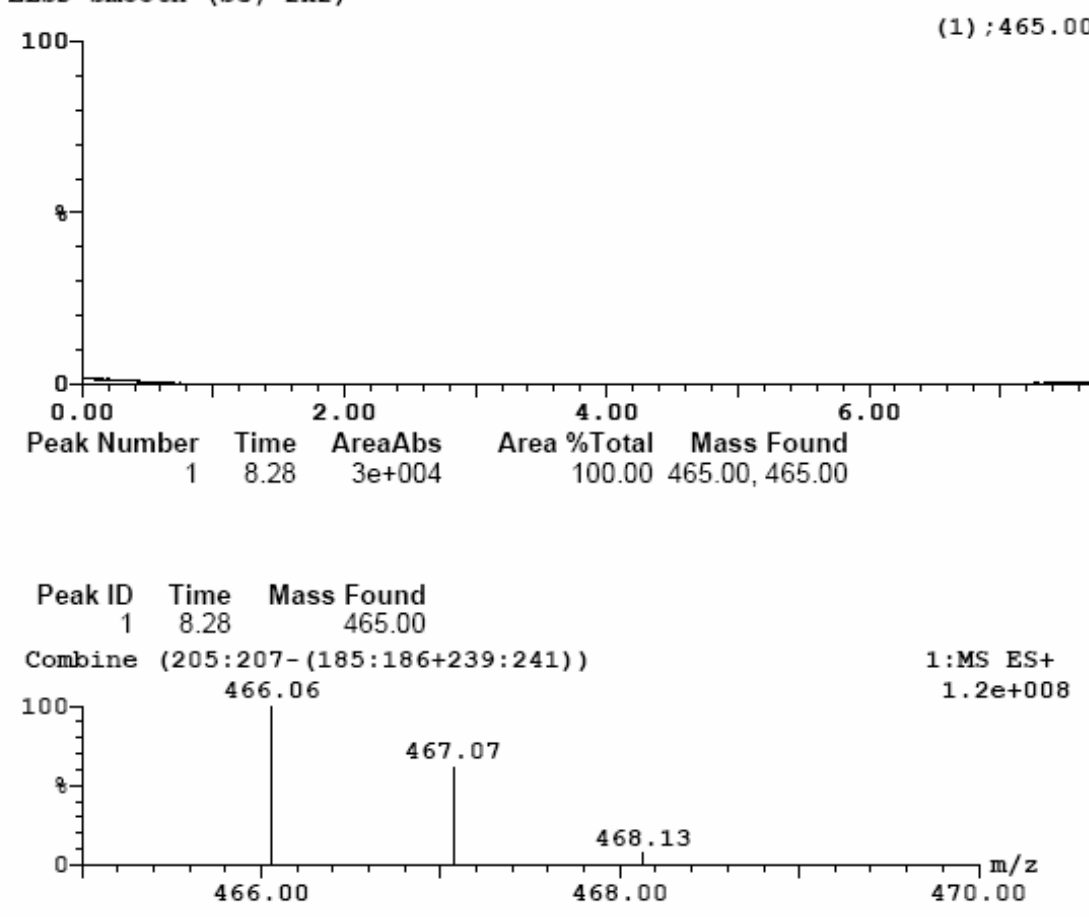

$7.3 e+004$

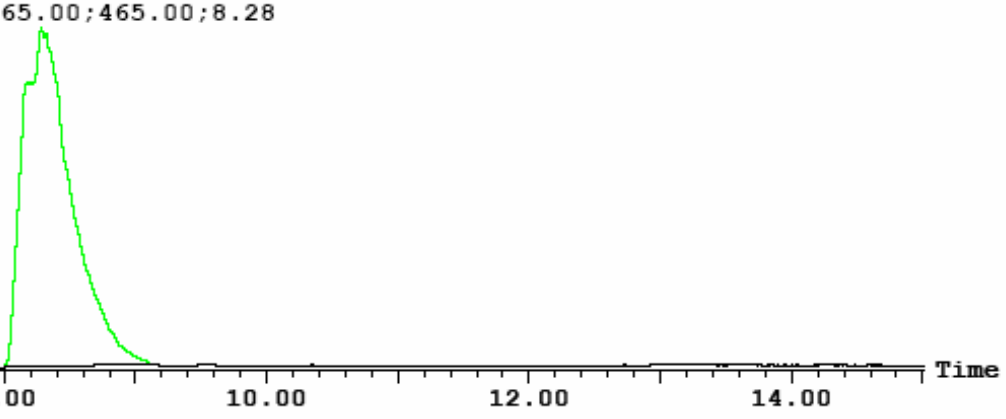

\section{SP-11}

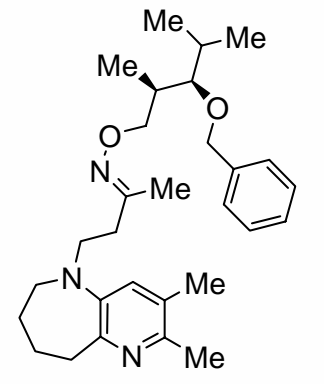

55 
Openlynx Report

Sample: 836

Vial:5,1:42

Date:30-Oct-2004

Page 14

File:SS-Lib-B8A14

Time:09-34:27

Printed: Fri Nov 05 11:25:43 2004

Sample Report (continued):

Sample 836 Vial 5,1:42 ID File SS-Lib-B8A14 Date 30-Oct-2004 Time 09:34:27 Description

ELSD Smooth (SG, 2x2)

100

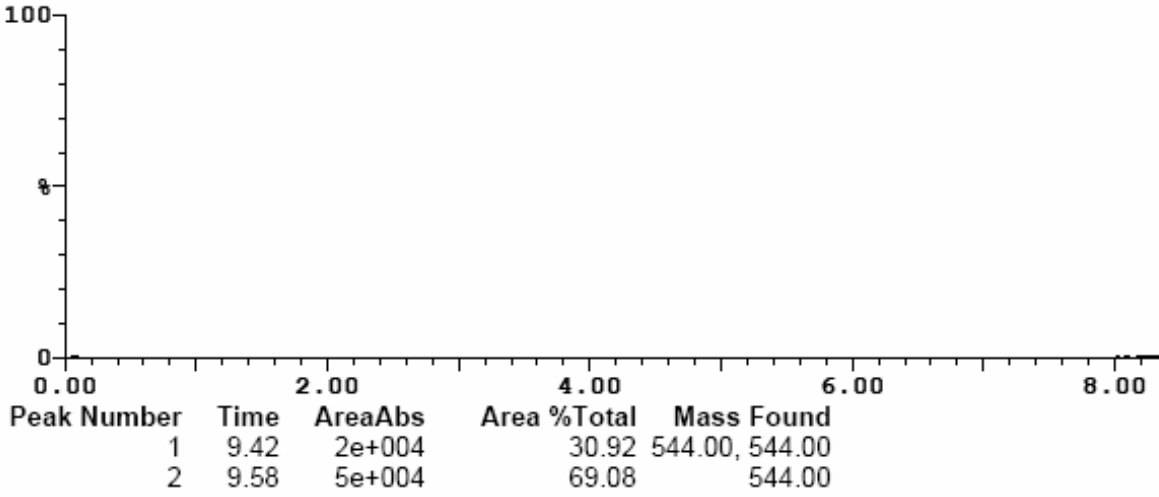

(2)

544.00

9.58

(1)

544.00

544.00

9.42

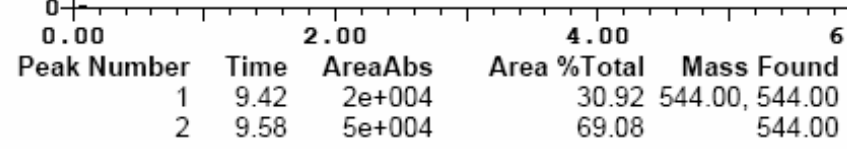

\section{$\begin{array}{rrr}\text { Peak ID } & \text { Time } & \text { Mass Found } \\ 1 & 9.42 & 544.00\end{array}$}

Combine $(233: 235-(218: 219+248: 249)) \quad 1:$ MS ES+

$1007545.06 \quad 6.4 \mathrm{e}+007$

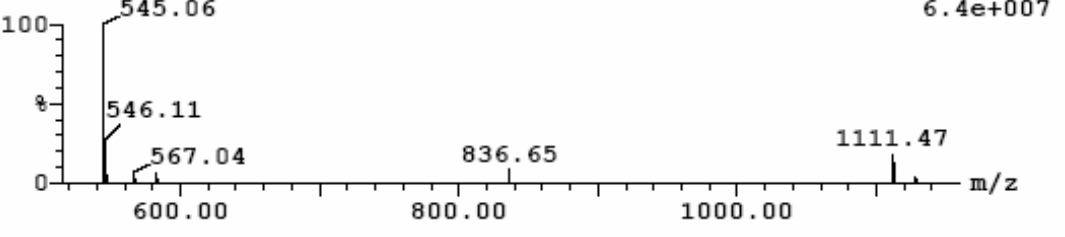

3. $9 e+005$

600.00 800.00 1000.00 


\begin{tabular}{lll}
\hline Openlynx Report - & & Page 2 \\
Sample: 718 & Vial:5,3:1 & ID: \\
File:SS-Lib-B1A1 & Date:20-Oct-2004 & Time:00:09:12 \\
Description: & & \\
Printed: Thu Nov 04 21:33:16 2004 & &
\end{tabular}

Printed: Thu Nov 04 21:33:16 2004

Sample Report:

Sample 718 Vial 5,3:1 ID File SS-Lib-B1A1 Date 20-Oct-2004 Time 00:09:12 Description

ELSD Smooth (SG, 2x2)

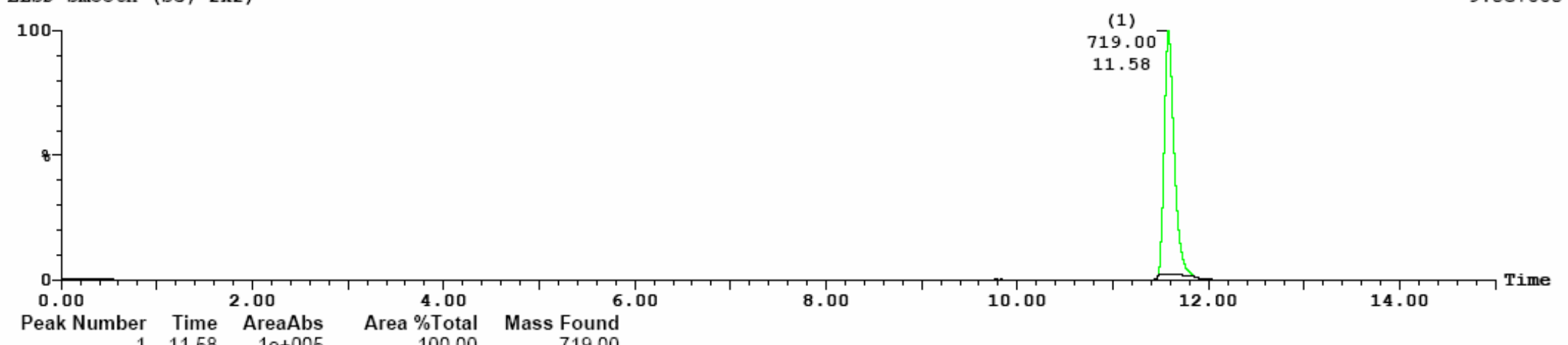

Peak Number Time AreaAbs Area \%Total Mass Foun

\section{Peak ID Time Mass Found}

Combine $(286: 289-(271: 273+306: 308))$

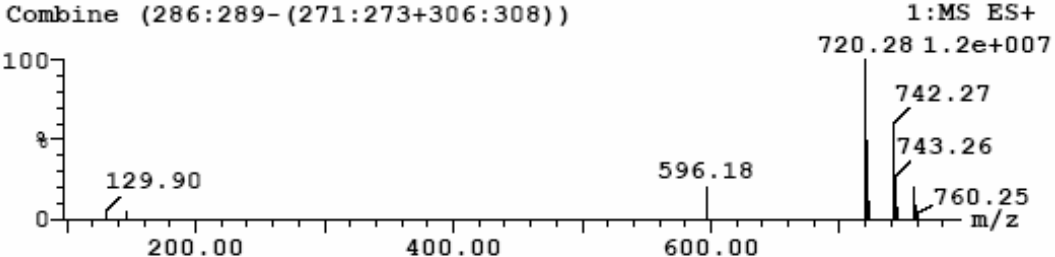

200.00

400.00
$.5 e+005$

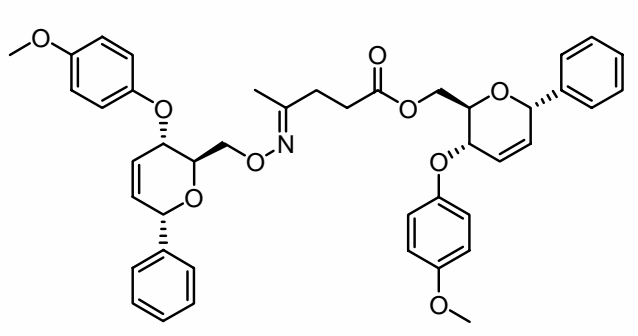

\section{SP-13}




\begin{tabular}{lll}
\hline Openlynx Report - & & Page 3 \\
Sample: 734 & Vial:5,3:16 & ID: \\
File:SS-Lib-B2A2 & Date:20-Oct-2004 & Time:04:55:56 \\
Description: & & \\
Printed: Thu Nov 04 21:37:19 2004 & &
\end{tabular}

Sample Report (continued):

Sample 734 Vial 5,3:16 ID File SS-Lib-B2A2 Date 20-Oct-2004 Time 04:55:56 Description

ELSD Smooth (SG，2x2)

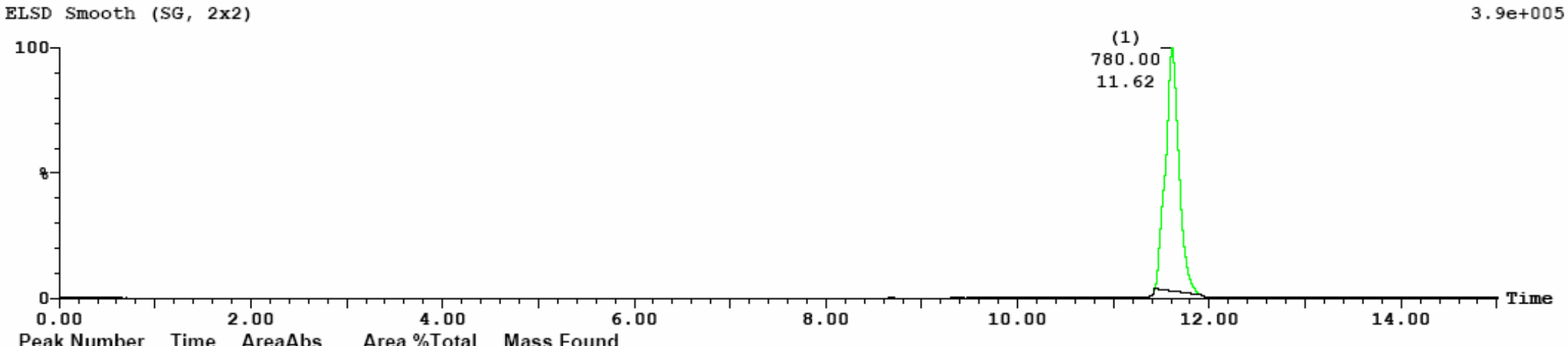

Peak Number Time AreaAbs Area \%Total Mass Found

$\begin{array}{rrr}\text { Peak ID } & \text { Time } & \text { Mass Found } \\ 1 & 11.62 & 780.00\end{array}$

Combine $(287: 290-(270: 272+308: 309)) \quad \begin{gathered}1: \text { MS ES+ } \\ 9.7 \mathrm{e}+006\end{gathered}$

Combine $(287: 290-(270: 272+308: 309)) \quad \begin{gathered}1: \text { MS ES+ } \\ 9.7 \mathrm{e}+006\end{gathered}$
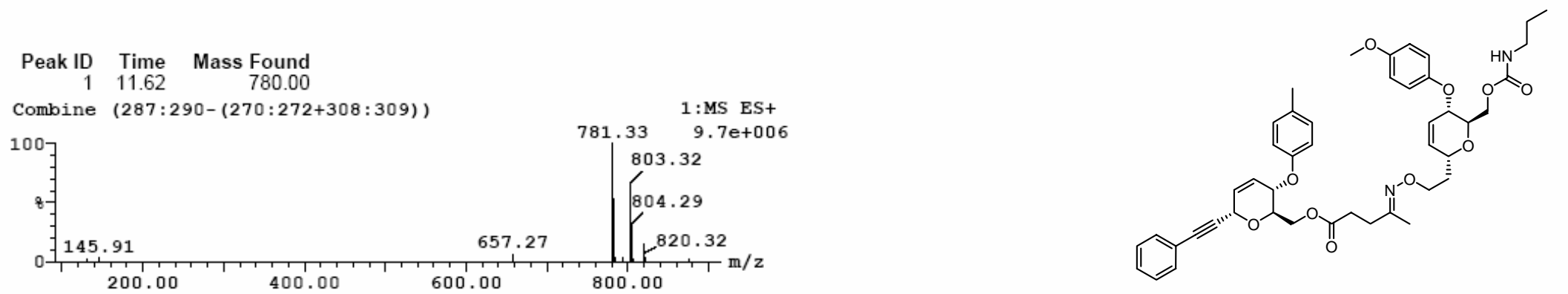
Openlynx Report

Sample: 750

File:SS-Lib-B3A3

Description:

Vial:5,3:31

Date:20-Oct-2004

ID:

Time:09:41:37

Printed: Thu Nov 04 21:45:04 2004

Sample Report (continued):

Sample 750 Vial 5,3:31 ID File SS-Lib-B3A3 Date 20-Oct-2004 Time 09:41:37 Description

ELSD Smooth (SG，2×2)

100

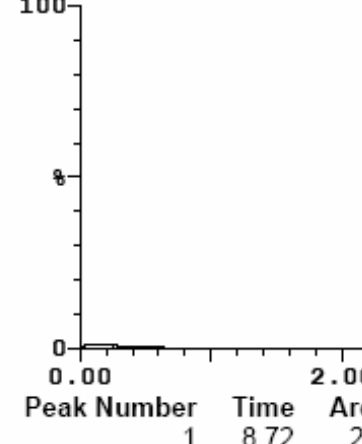

Peak Number Time AreaAbs

$\begin{array}{rr}\text { Time } & \text { AreaAbs } \\ 8.72 & 2 \mathrm{e}+004\end{array}$

(1)

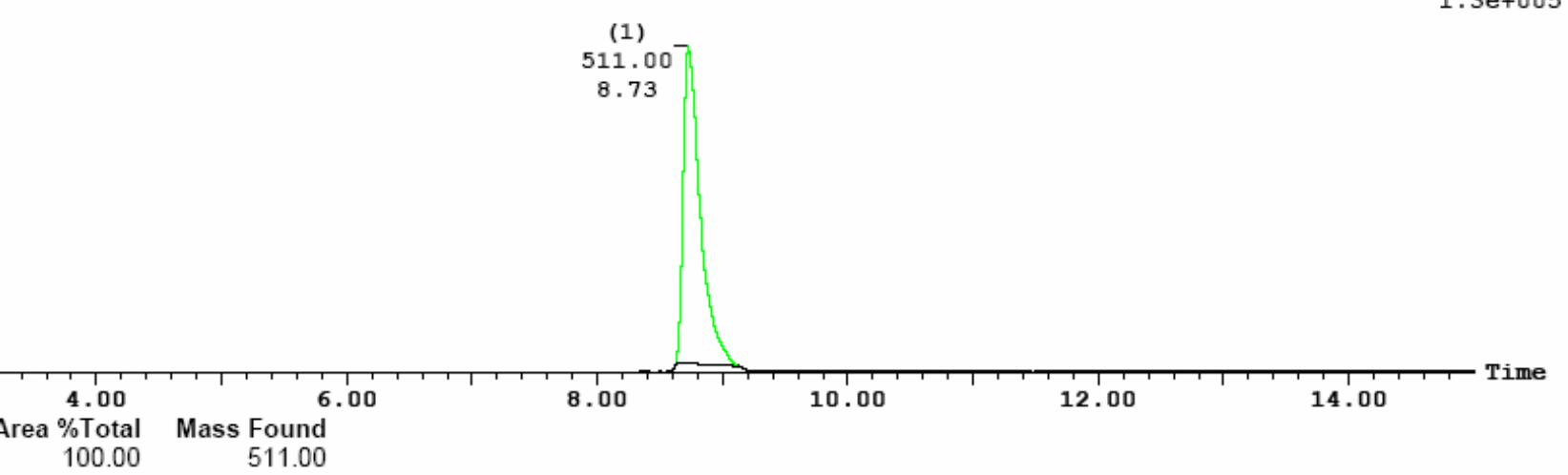

$\begin{array}{rrr}\text { Peak ID } & \text { Time } & \text { Mass Found } \\ 1 & 8.72 & 511.00\end{array}$

Combine $(216: 218-(201: 202+239: 240))$

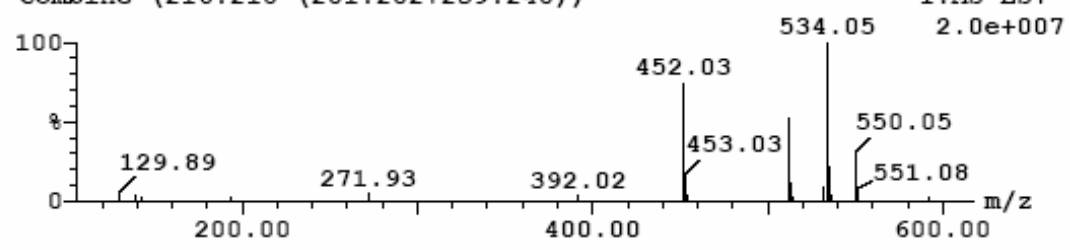

Page 4 
Openlynx Report -

Sample: 768

File:SS-Lib-B4A6

Vial:5,1:4

Date:24-Oct-2004

Description:

ID:

Time:21:47:50

Printed: Fri Nov 05 11:21:54 2004

Sample Report (continued):

Sample 768 Vial 5,1:4 ID File SS-Lib-B4A6 Date 24-Oct-2004 Time 21:47:50 Description

ELSD Smooth (SG，2×2)

$1.1 e+005$

100

$$
\text { (1) }
$$

463.007

$$
\begin{aligned}
& 463.00 \\
& 463.00
\end{aligned}
$$

$$
4.46
$$

8

$-$

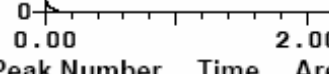

Peak Number Time AreaA

$\begin{array}{rrr}1 & \text { Time } & \text { AreaAbs } \\ & 4.46 & 4 \mathrm{e}+004\end{array}$

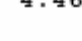

$\begin{array}{rrr}\text { Peak ID } & \text { Time } & \text { Mass Found } \\ 1 & 4.46 & 463.00\end{array}$

Combine (110:112-(93:94+143:144))

1007464.04

$1:$ MS ES+

1. $2 \mathrm{e}+008$
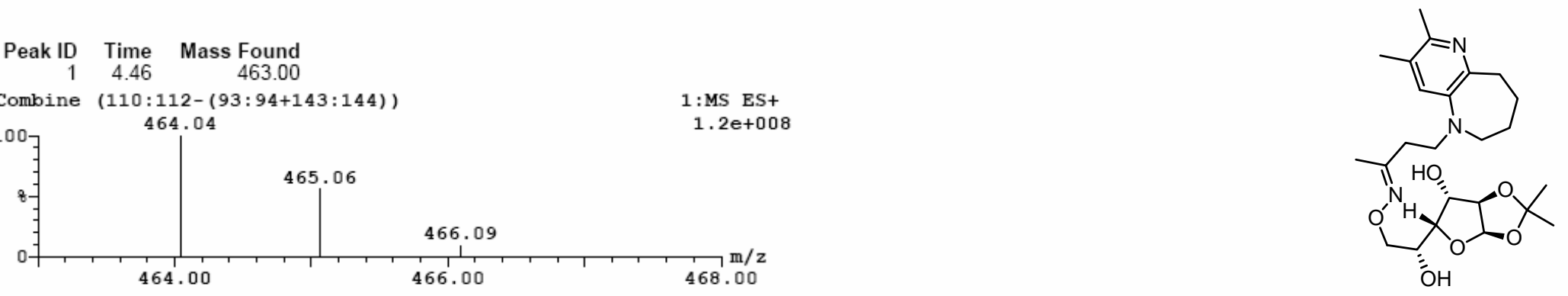


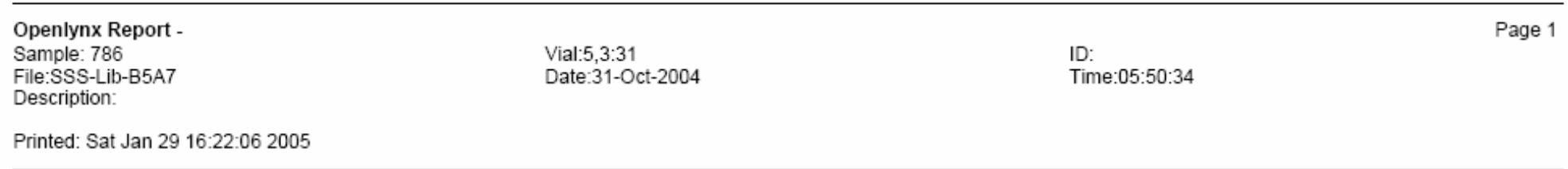

Sample Report:

Sample 786 Vial 5,3:31 ID File SSS-Lib-B5A7 Date 31-Oct-2004 Time 05:50:34 Description ELSD Smooth (SG, 3x3)

$2.1 e+005$

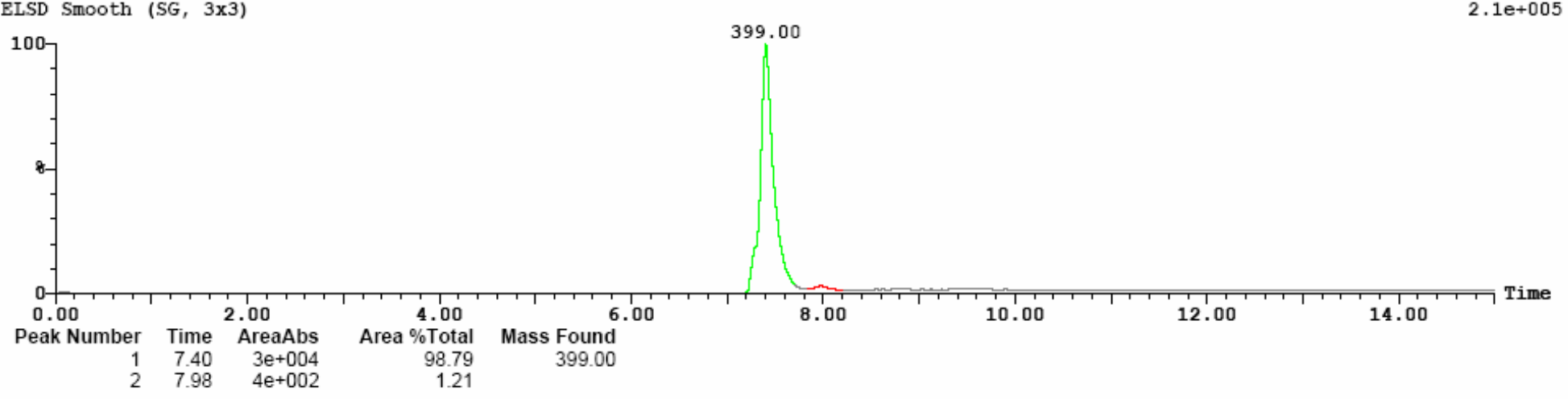

Peak ID Time Mass Found
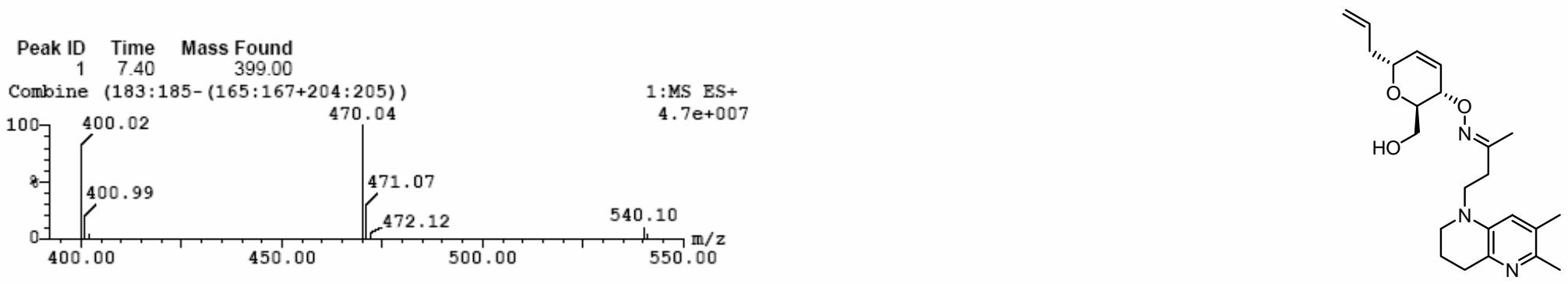


\begin{tabular}{lll}
\hline Openlynx Report - & & Page 9 \\
Sample: 800 & Vial:5,1:8 & ID: \\
File:SS-Lib-B6A8 & Date:21-Oct-2004 & Time:03:21:27
\end{tabular}

Description:

Printed: Thu Nov 04 22:28:55 2004

Sample Report (continued):

Sample 800 Vial 5,1:8 ID File SS-Lib-B6A8 Date 21-Oct-2004 Time 03:21:27 Description

ELSD Smooth (SG，2x2)
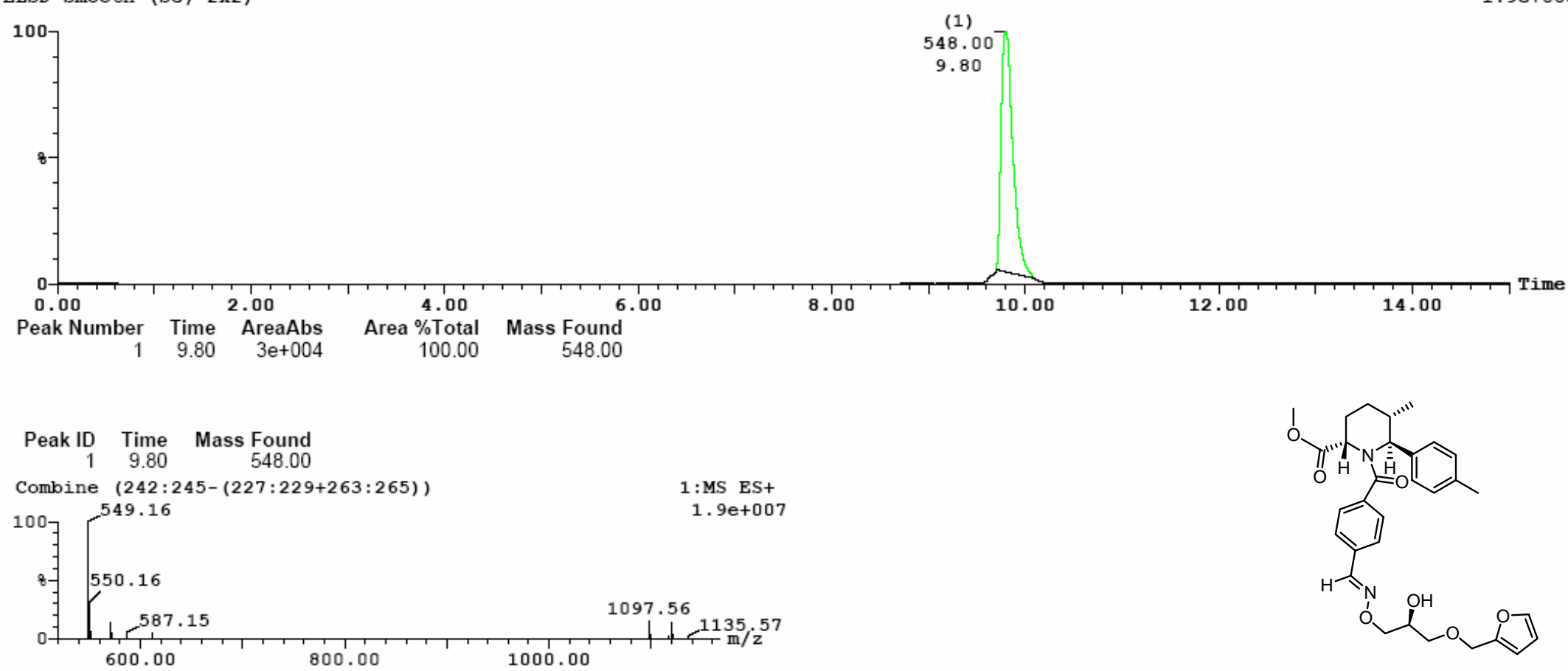
Openlynx Report -

Sample: 816

Vial: $5,1: 4$

Date:30-Oct-2004

Time: $18: 45: 56$

Printed: Thu Nov 04 22:27:51 2004

Sample Report (continued):

Sample 816 Vial 5,1:4 ID File SSS-Lib-B7A9 Date 30-Oct-2004 Time 18:45:56 Description

ELSD Smooth (SG，2×2)

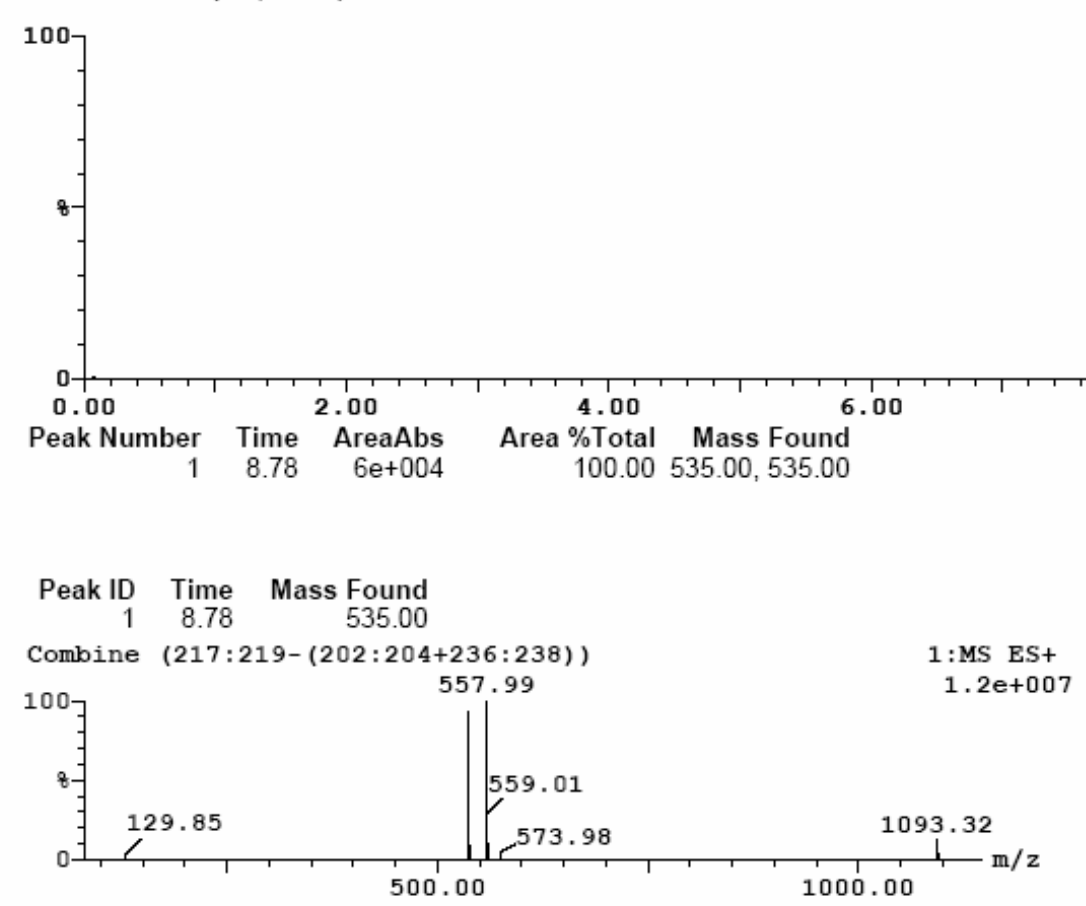

$5.1 e+005$ (1) 535.00 535.00
535.00 8.78

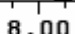

12.00

14.00

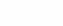

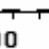

00

(2)

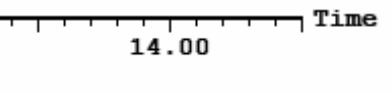


Printed: Fri Nov 05 11:25:43 2004

Sample Report (continued):

Sample 832 Vial 5,1:38 ID File SS-Lib-B8A10 Date 27-Oct-2004 Time 17:38:50 Description

ELSD Smooth (SG，2x2)

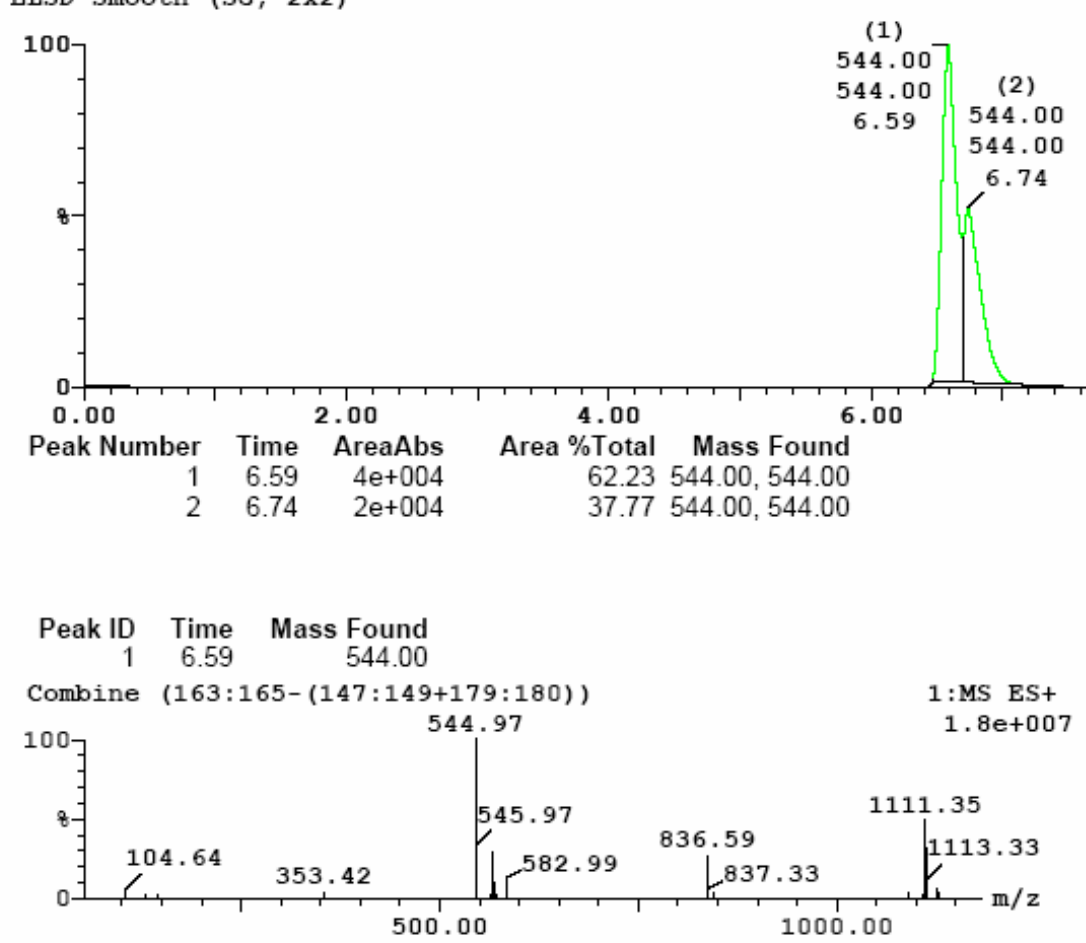$$
\text { 6223 } 54400,5440
$$

1000.00
$3.2 e+005$

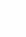

14.00

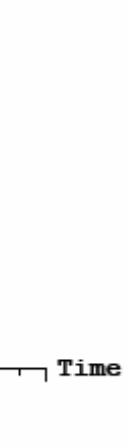

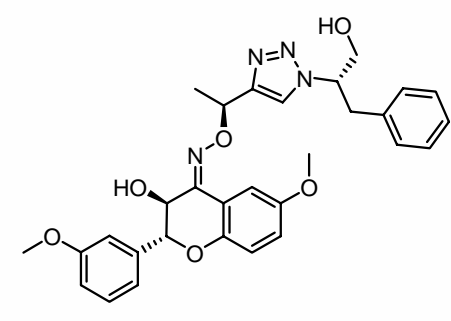


Openlynx Report -

Sample: 848

Vial:5, $1: 11$

Date: $30-$ Oct-2004

Page 12

Description:

Printed: Fri Nov 05 11:36:16 2004

Sample Report (continued):

Sample 848 Vial 5,1:11 ID File SS-Lib-B9A11 Date 30-Oct-2004 Time 01:57:13 Description

ELSD Smooth (SG, 2x2)

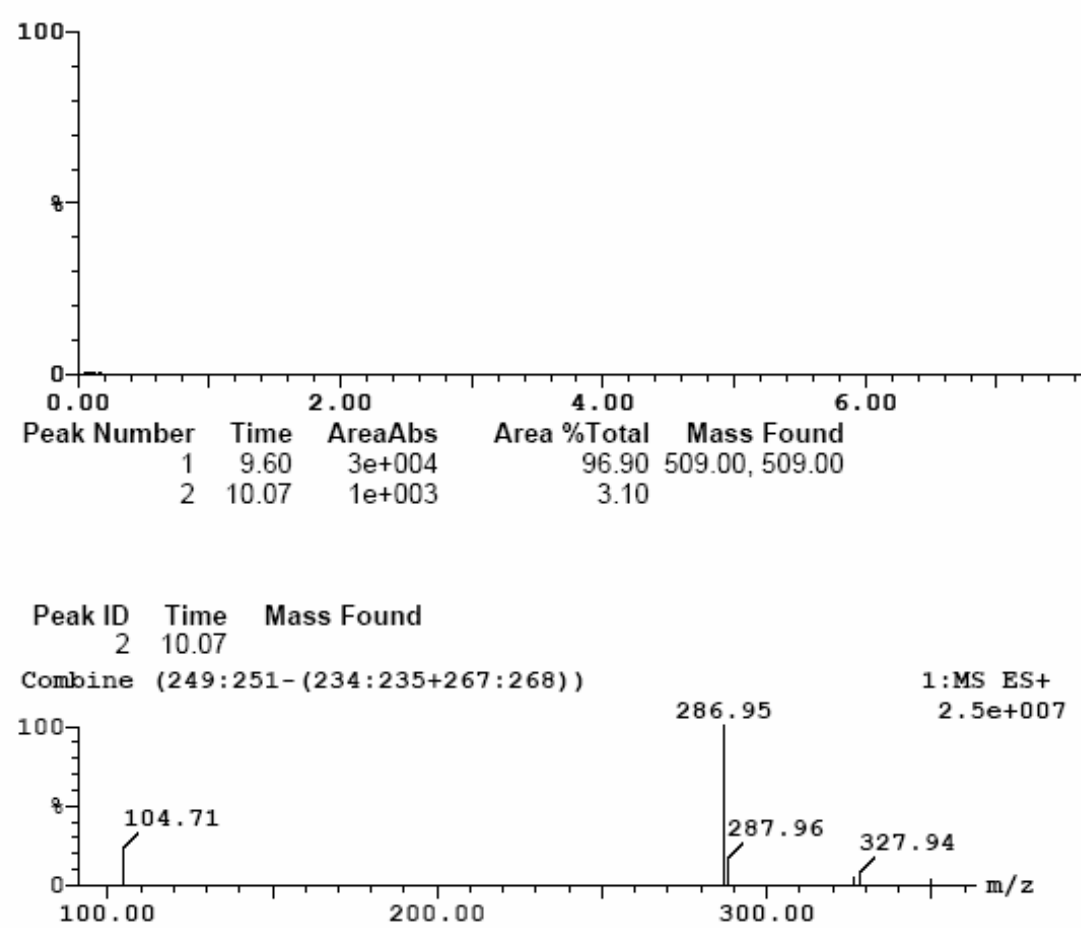

$2 \cdot 2 e+005$

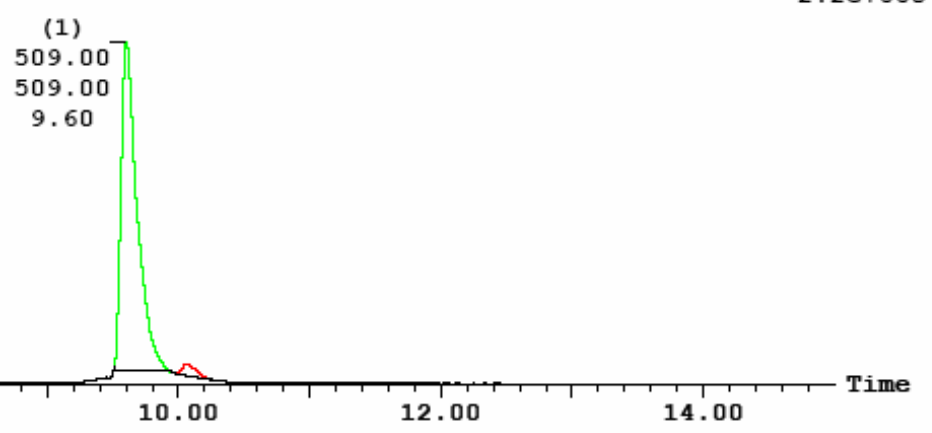

509.00

12.00

8.00

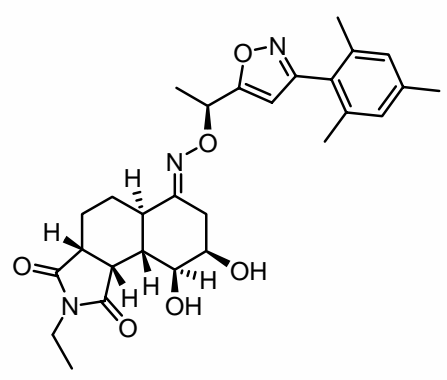


Openlynx Report -

Sample: 864

File:SS-Lib-B10A12

Description:

Printed: Fri Nov 05 12:12:00 2004

Sample Report (continued):

Sample 864 Vial 5,1:26 ID File SS-Lib-B10A12 Date 30-Oct-2004 Time 07:01:47 Description

ELSD Smooth (SG，2x2)

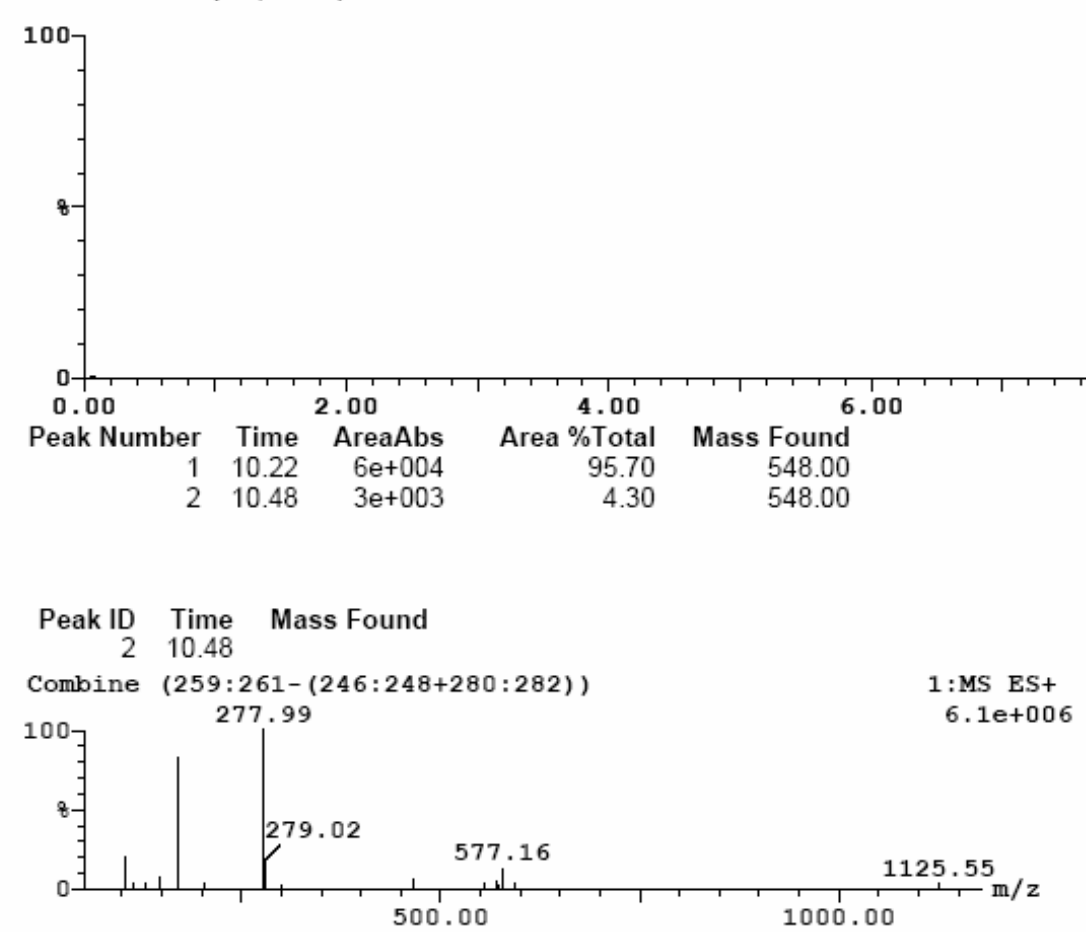

Vial:5, 1:26

Date:30-Oct-2004 
Printed: Fri Nov 05 12:12:57 2004

Sample Report (continued):

Sample 880 Vial 5,1:13 ID File SS-Lib-B11A13 Date 21-Oct-2004 Time 19:52:08 Description

ELSD Smooth $(\mathrm{SG}, 2 \times 2)$

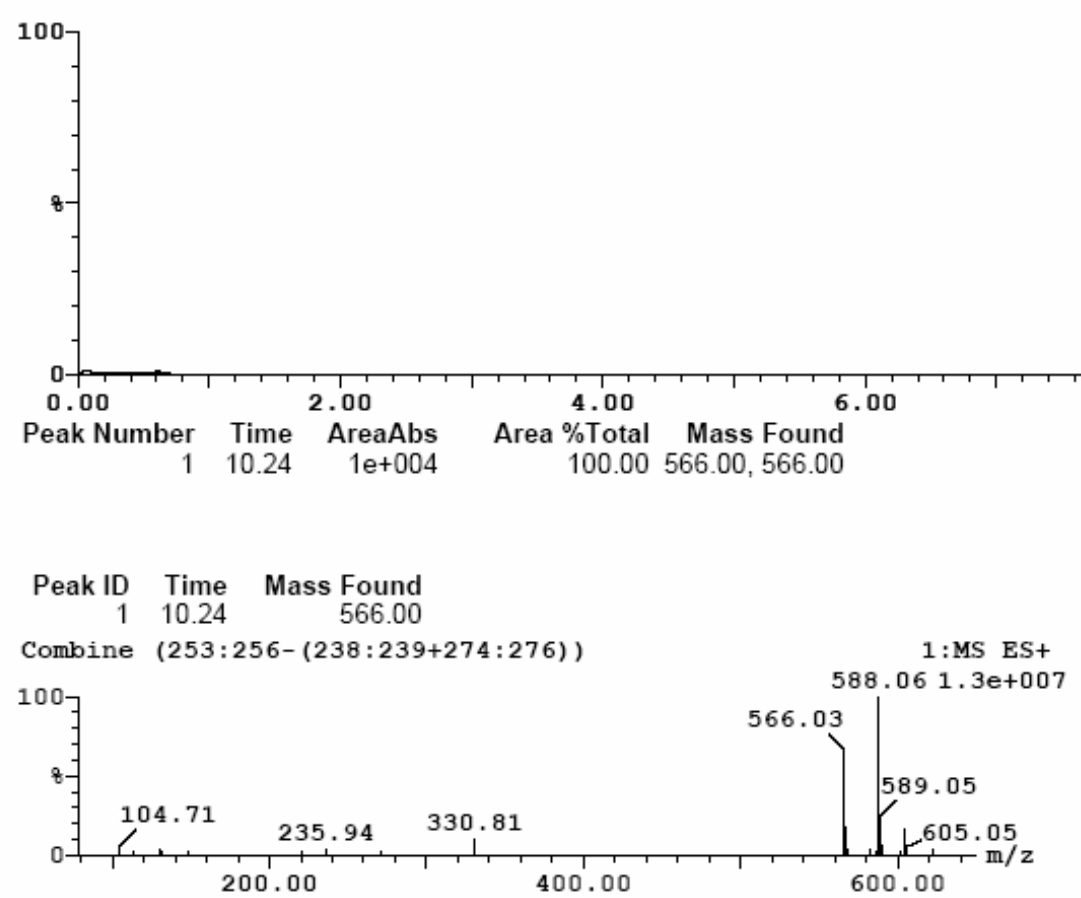

Time:19:52:08 
Openlynx Report -

Sample: 895

File:SSS-Lib-B12A13

Description:

Printed: Fri Nov 05 17:57:43 2004

Sample Report (continued):

Sample 895 Vial 5,3:24 ID File SSS-Lib-B12A13 Date 31-Oct-2004 Time 03:41:20 Description

ELSD Smooth (SG，2x2)

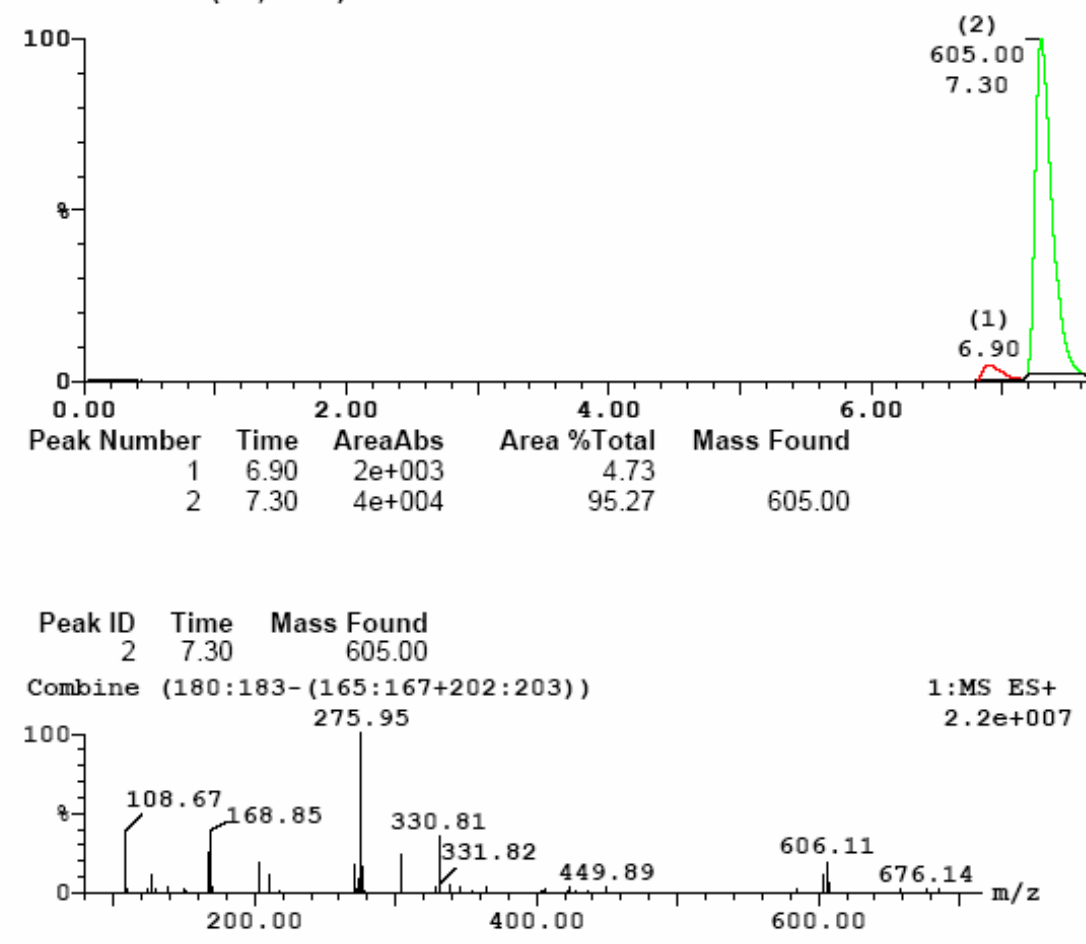

Page 14

Time:03:41:20

20




\section{Printed: Fri Nov 05 11:20:08 2004}

Sample Report (continued):

Sample 903 Vial 5,3:6 ID File SSS-Lib-B13A6 Date 30-Oct-2004 Time 23:27:16 Description

ELSD Smooth $(\mathrm{SG}, 2 \times 2$

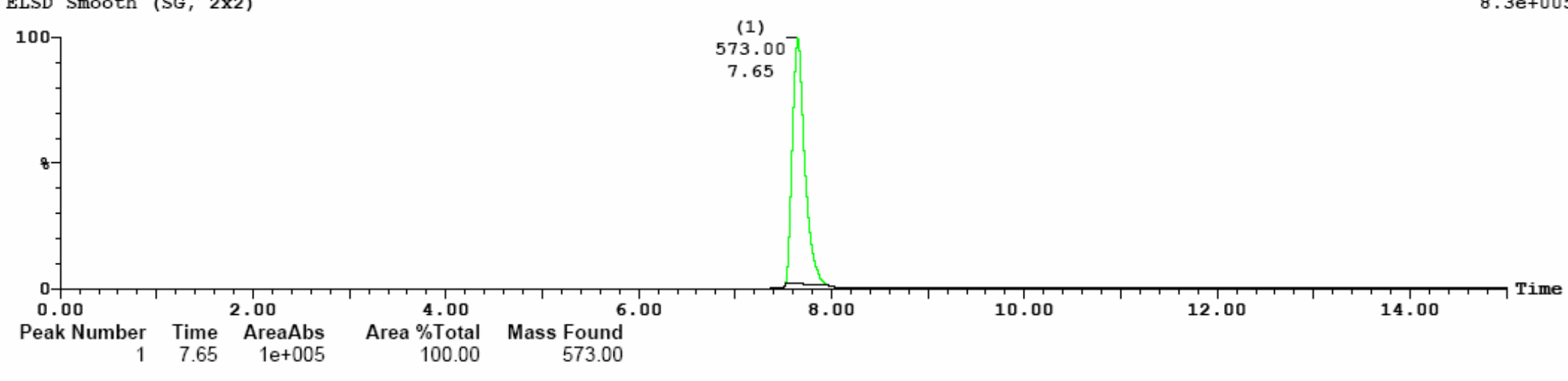

$\begin{array}{rrr}\text { Peak ID } & \text { Time } & \text { Mass Found } \\ 1 & 7.65 & 573.00\end{array}$

Combine $(189: 191-(173: 175+210: 211))$

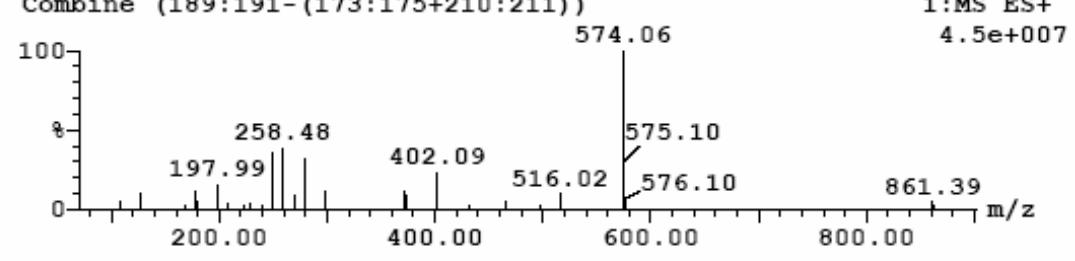

ID:
Time:23:27:16 
Printed: Fri Nov 05 11:14:53 2004

Sample Report (continued):

Sample 926 Vial 5,3:14 ID File SS-Lib-B14A14 Date 26-Oct-2004 Time 23:21:28 Description

ELSD Smooth (SG, 2x2

100

$$
\text { (1) }
$$

444.00

444.00

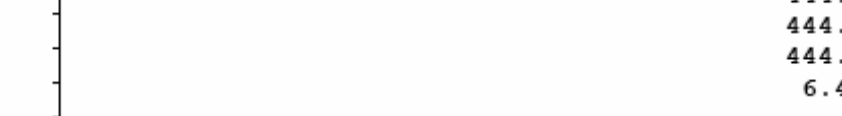

$$
8
$$
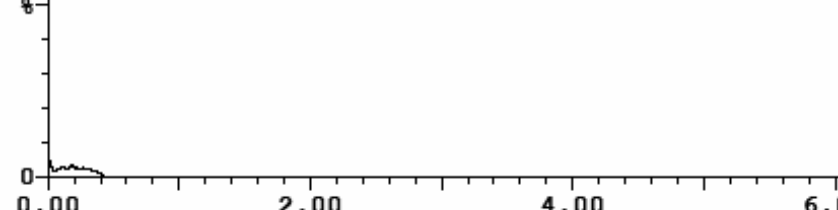

$\begin{array}{cccc}0.00 & 2.00 & 4.00 & 6 . \\ \text { Peak Number } & \text { Time } & \text { AreaAbs } & \text { Area } \% \text { Total Mass Found }\end{array}$

$$
6.40
$$

\section{$\begin{array}{rrr}\text { Peak ID } & \text { Time } & \text { Mass Found } \\ 1 & 6.40 & 444.00\end{array}$}

Combine (158:160-(140:142+179:181))
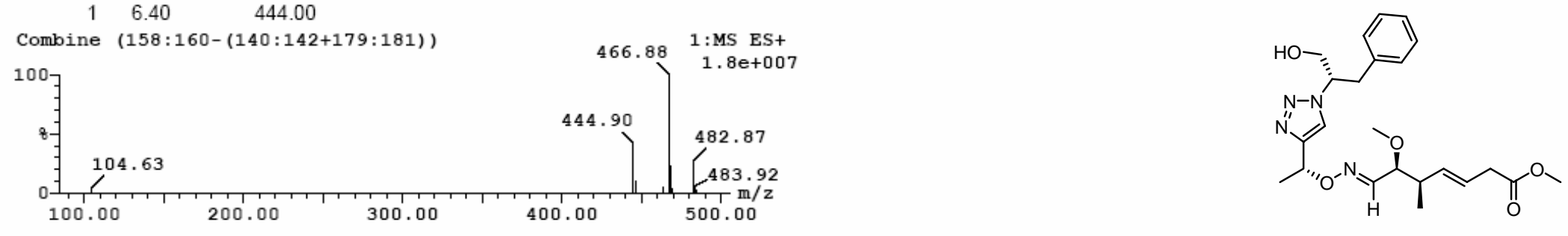


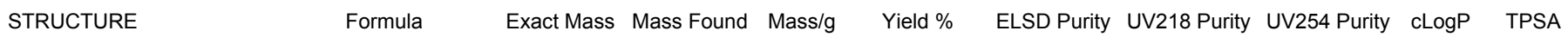

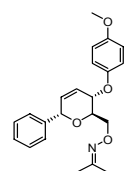

$\begin{array}{llllllllll}\mathrm{C} 22 \mathrm{H} 25 \mathrm{NO} 4 & 367.1784 & 367.9 & 0.0063 & 90.5 & >99 \% & \mathrm{~N} / \mathrm{A} & \mathrm{N} / \mathrm{A} & 5.75 & 49.30\end{array}$

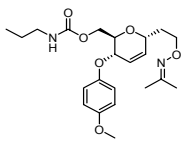

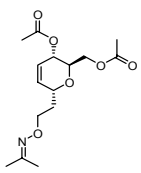

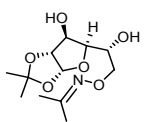<smiles>CC(C)CC(C)C</smiles><smiles>C1CCOC1</smiles>

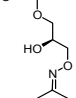<smiles>CC(C)=C(C)C1=NC1NC1CO1</smiles><smiles>CC(C)CC1CCCC1C(C)(C)C</smiles>

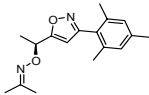<smiles>CC(C)CC(C)OC(C)(C)C1CCCCC1</smiles>

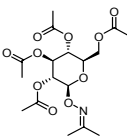

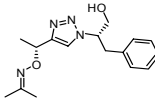

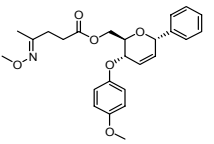

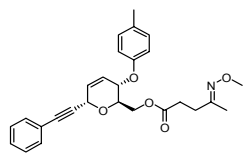

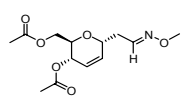

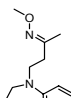

C22H32N2O6

420.2260

421

0.0078

97.9

$>99 \%$

N/A

N/A

$5.10 \quad 87.63$

C15H23NO6

313.1525

314.

0.0055

92.7

$>99 \%$

$91.9 \%$

N/A

$2.31 \quad 83.44$

C12H21NO6

275.1369

275.

0.0050

95.9

$98.5 \%$

N/A

$0.42 \quad 89.76$

226.

0.0042

98.4

N/A

$85.5 \%$

N/A

$2.54 \quad 51.06$

C11H17NO

227.115

228

0.004

92.9

N/A

$85.9 \%$

N/A

$2.15 \quad 64.20$

C11H18N4

254.1379

254

0.0046

95.5

$98.8 \%$

$92.6 \%$

NIA

$0.95 \quad 81.78$

$\mathrm{C} 16 \mathrm{H} 22 \mathrm{~N} 4$

C17H22N2

277.204

278

0.0050

95.2

N/A

$>99 \%$

N/A

$5.11-30.83$

403.1478

403

0.0075

98.2

$96.5 \%$

N/A

N/A

$1.09 \quad 136.05$

$\mathrm{C} 16 \mathrm{H} 22 \mathrm{~N} 4 \mathrm{O}$

302.174

302

0.0057

99.5

$>99 \%$

$>99 \%$

N/A

$3.20 \quad 72.54$

$\mathrm{C} 25 \mathrm{H} 29 \mathrm{NO}$

439.199

440

0.008

97.3

$>99 \%$

$>99 \%$

$95.6 \%$

$5.69 \quad 75.61$

C27H29NO5

447.2046

447.

0.0081

95.6

$>99 \%$

$>99 \%$

$>99 \%$

$5.87 \quad 66.37$

C13H19NO

285.121

285

C16H25N3O

275.1998 $\begin{array}{lll}275.9 & 0.0052 \quad 99.7\end{array}$

$\begin{array}{lll}275.9 & 0.0052 \quad 99.7\end{array}$
N/A

$>99 \%$
N/A

$>99 \%$ 
STRUCTURE

$\int_{n=1}^{\circ}$

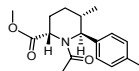

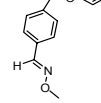

-
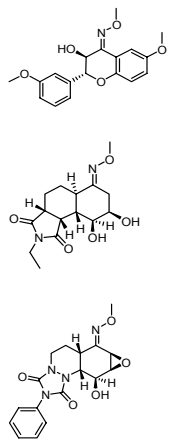

10

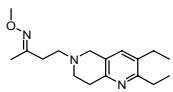

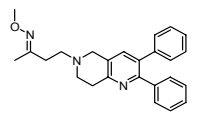<smiles>CCCCC(C)C(C)CCCC(C)C</smiles>

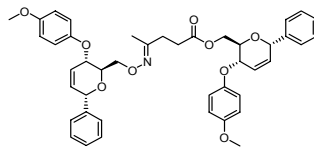

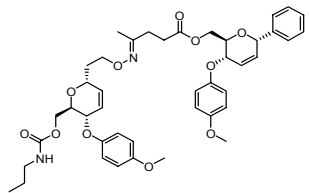

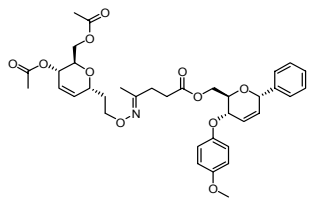

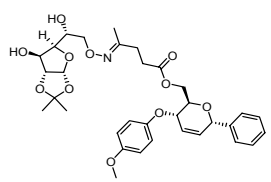

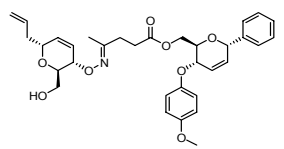

\begin{abstract}
Formula
\end{abstract}
Exact Mass Mass Found Mass/g

Yield \%

ELSD Purity UV218 Purity UV254 Purity cLogP TPSA

C15H23N3O

261.1841

261.

0.0049

99.0

$95.2 \%$

N/A

$88.7 \%$

$3.52 \quad 37.73$

$\mathrm{C} 24 \mathrm{H} 28 \mathrm{~N} 2 \mathrm{O}$

408.2049

408.9

0.0074

95.6

$>99 \%$

$>99 \%$

$>99 \%$

$4.55 \quad 68.21$

C17H24N2O5S

368.1406

368

0.0051

72.4

$96.8 \%$

N/A

N/A

$2.28 \quad 94.07$

C18H19NO5

329.1263

329.9

0.0051

81.0

$>99 \%$

$>99 \%$

$\begin{array}{lll}86.4 \% & 3.07 \quad 69.53\end{array}$

C15H

310.1529

311.

0.0056

95.3

$>99 \%$

$>99 \%$

N/A

$0.18 \quad 99.43$

C17H18N405

358.1277

359.1

0.0063

92.2

$>99 \%$

$>99 \%$

$>99 \%$

$0.79 \quad 103.30$

$\mathrm{C} 15 \mathrm{H} 23 \mathrm{NO} 2$

249.1729

250.

0.0044

93.2

N/A

$91.2 \%$

N/A

$3.71 \quad 30.83$

C17H27N3O

289.215

289

0.0052

94.9

$98.6 \%$

N/A

$91.9 \%$

$3.42 \quad 37.73$

$\mathrm{C} 25 \mathrm{H} 27 \mathrm{~N} 3 \mathrm{O}$

385.215

385.

0.0072

98.6

$98.3 \%$

C11H19NO4

229.1314

254.

0.0043

99.0

N/A

C43H45NO9

719.3094

720

0.0126

92.1

$>99 \%$

$>99 \%$

$>99 \%$

$>99 \%$

$5.50 \quad 37.73$

C43H52N2O11

772.357

773.

0.0141

96.3

$>99 \%$

$>99 \%$

$98.0 \%$

$8.87 \quad 141.64$

C36

665.2836

666.2

0.0113

89.7

$>99 \%$

$>99 \%$

$97.2 \%$

$6.30 \quad 137.45$

$\mathrm{C} 33 \mathrm{H} 41 \mathrm{NO} 11$

627.2680

628.1

0.0105

88.3

$>99 \%$

$>99 \%$

N/A

$4.41 \quad 143.76$

C33H39NO8

577.2676

578.

0.0097

88.7
$>99 \%$ 
STRUCTURE

OC

Formula

606.2690

$\mathrm{C} 37 \mathrm{H} 42 \mathrm{~N} 4 \mathrm{O} 7$

654.3054

655

$\mathrm{C} 38 \mathrm{H} 42 \mathrm{~N} 2 \mathrm{O}$

638.2992

$639.2 \quad 0.0114$

94.2

$98.8 \%$

$95.9 \%$

$94.5 \%$

$9.44 \quad 101.64$

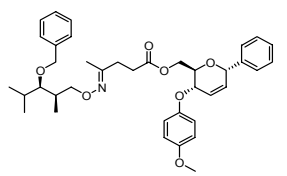

$\mathrm{C} 38 \mathrm{H} 47 \mathrm{NO} 7$

629.3353

630

0.0110

92.2

$>99 \%$

$>99 \%$

$94.5 \%$

8.88

84.84

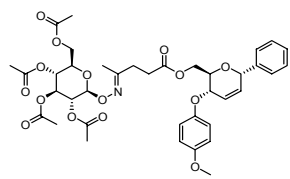

$\mathrm{C} 38 \mathrm{H} 45 \mathrm{NO} 15$

755.278

756

0.0142

99.2

$>99 \%$

$89.9 \%$

$93.7 \%$

$5.08 \quad 190.06$

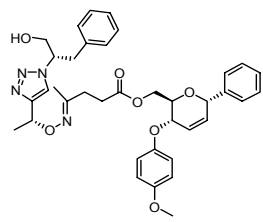

654.3054

655.

0.0118

95.2

$>99 \%$

$96.0 \%$

$94.2 \%$

$6.97 \quad 126.55$

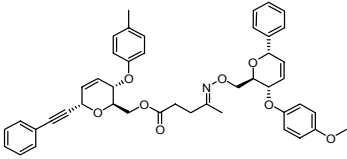

$\mathrm{C} 45 \mathrm{H} 45 \mathrm{NO} 8$

727.3145

728

0.0134

97.2

$>99 \%$

$96.1 \%$

$95.7 \%$

$9.71 \quad 94.07$

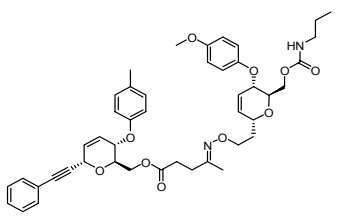

C45H52N2O10

780.3622

781

0.0147

99.4

$>99 \%$

$>99 \%$

$>99 \%$

9.0

132.41

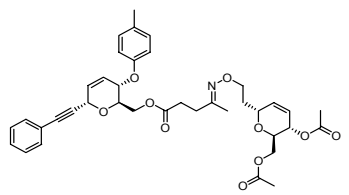

C38H43NO10

673.2887

674

0.0119

93.3

$>99 \%$

$>99 \%$

$>99 \%$

$6.49 \quad 128.22$

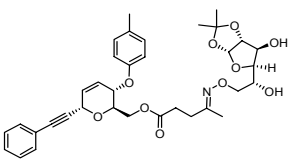

$\mathrm{C} 35 \mathrm{H} 41 \mathrm{NO}$

635.2730

636.1

0.011

95.5

$>99 \%$

$>99 \%$

$>99 \%$

$4.60 \quad 134.53$

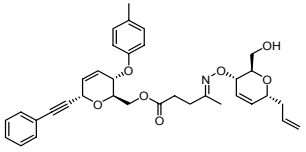

585.2727

586

0.0110

99.2

$>99 \%$

$>99 \%$

$98.4 \%$

\begin{tabular}{ll}
95.83 \\
\hline
\end{tabular} 


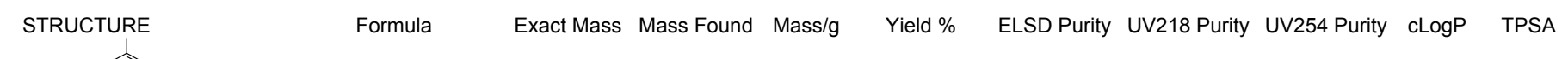
I) C34H37NO8 91.6 $>99 \%$ $>99 \%$ $6.11 \quad 108.97$

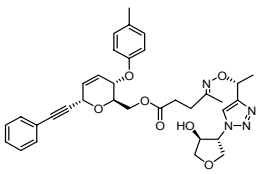
C34H38N4O7 614.2741 $615.2 \quad 0.0113$

97.1

$>99 \%$

$>99 \%$

$>99 \%$

$4.91 \quad 126.55$

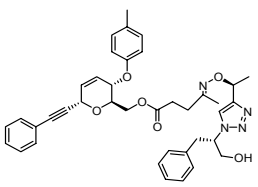

$\mathrm{C} 39 \mathrm{H} 42 \mathrm{~N} 4 \mathrm{O} 6$

662.3104

663.3

0.0125

99.6

$>99 \%$

$>99 \%$

$>99 \%$

$7.16 \quad 117.32$

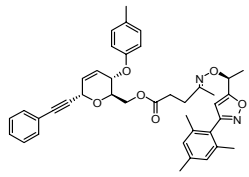

$\mathrm{C} 40 \mathrm{H} 42 \mathrm{~N} 2 \mathrm{O} 6$

646.3043

647

0.0112

91.4

$>99 \%$

$90.0 \%$

$91.1 \%$

$9.63 \quad 92.40$

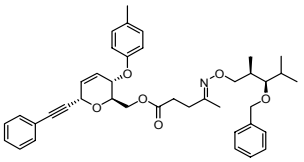

C40H47NO6

637.3403

638.3

0.0118

97.7

$>99 \%$

$>99 \%$

$97.9 \%$

$9.07 \quad 75.61$

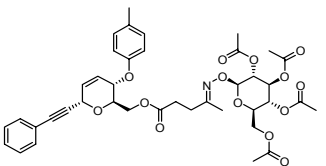

$\mathrm{C} 40 \mathrm{H} 45 \mathrm{NO} 14$

763.2840

764.

0.0134

92.6

$>99 \%$

$97.4 \%$

$96.6 \%$

$5.27 \quad 180.83$

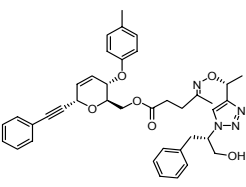

$\mathrm{C} 39 \mathrm{H} 42 \mathrm{~N} 4 \mathrm{O} 6$

662.3104

663.3

0.0120

95.6

$>99 \%$

$97.1 \%$

$95.3 \%$

$7.16 \quad 117.32$

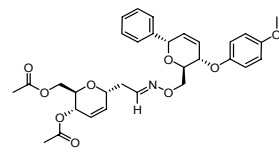

C31H35NO9

566

0.0097

90.5

$>99 \%$

$>99 \%$

N/A

$4.97 \quad 111.14$

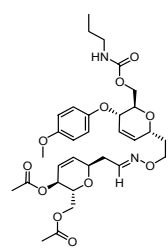

C31H42N2O11

618.2789

619

0.0116

99.0

$>99 \%$

$>99 \%$

N/A

$4.32 \quad 149.48$

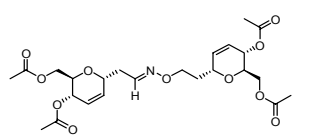

$\mathrm{C} 24 \mathrm{H} 33 \mathrm{NO} 11$

512

0.0091

93.9

$>99 \%$

N/A

N/A

$1.53 \quad 145.29$

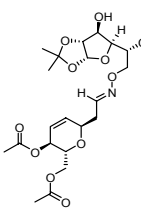

C21H31NO11

473.189

474.

0.008

91.

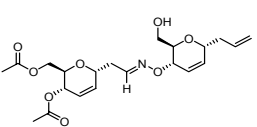

C21H29NO8

423.1893

424.0

0.0079

98.5

$>99 \%$

$>99 \%$

N/A

$1.76 \quad 112.90$ 
STRUCTURE Formula Exact Mass Mass Found Mass/g Yield \% ELSD Purity UV218 Purity UV254 Purity cLogP TPSA

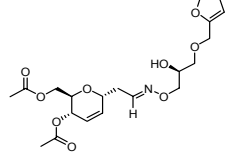

$\begin{array}{llllllllll}\mathrm{C} 2 \mathrm{H} 27 \mathrm{NO} & 425.1686 & 426.0 & 0.0078 & 96.8 & >99 \% & \mathrm{~N} / \mathrm{A} & \mathrm{N} / \mathrm{A} & 1.37 & 126.04\end{array}$

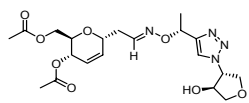

$\mathrm{C} 2 \mathrm{OH} 28 \mathrm{~N} 4 \mathrm{O} 8$

$\begin{array}{lllll}452.1907 & 453.1 & 0.0080 & 93.4 & >99 \%\end{array}$

N/A

N/A

$0.17 \quad 143.62$

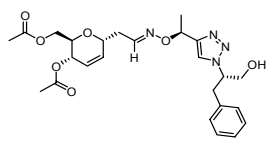

500.2271

501.

0.0094

99.2

$>99 \%$

N/A

N/A

$2.42 \quad 134.39$

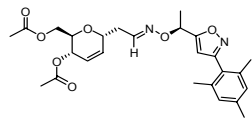

485.1

0.0091

99.2

$>99 \%$

$92.0 \%$

$93.3 \%$

$4.89 \quad 109.47$

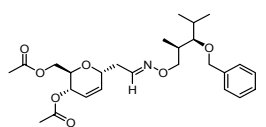

$\mathrm{C} 26 \mathrm{H} 37 \mathrm{NO} 7$

475.2570

476.

0.0086

$95.5 \quad>99 \%$

$96.1 \%$

N/A

$4.33 \quad 92.68$

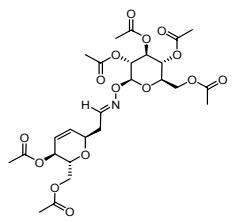

$\mathrm{C} 26 \mathrm{H} 35 \mathrm{NO}$

601.2007

602.2

0.0112

98.3

$>99 \%$

N/A

N/A

$0.31 \quad 197.90$

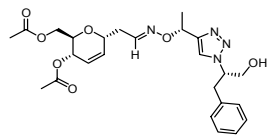

$\mathrm{C} 25 \mathrm{H} 32 \mathrm{~N} 4 \mathrm{O}$

500.227

501

0.008

90.7

$>99 \%$

$96.5 \%$

N/A

$2.42 \quad 134.39$

"10.

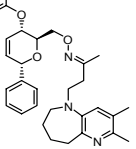

$\mathrm{C} 34 \mathrm{H} 41 \mathrm{~N} 3 \mathrm{O} 4$

555.3097

556.1

0.0104

98.8

$>99 \%$

$93.3 \%$

$98.2 \%$

$8.00 \quad 65.43$

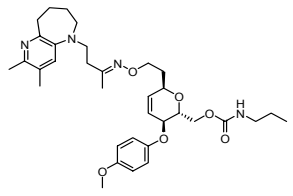

C34H48N4O6

608.3574

609.

0.0113

98.0

$98.7 \%$

$93.5 \%$

$97.7 \%$

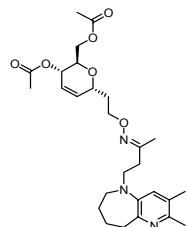

C27H39N3O6

502

90.5

$>99 \%$

N/A

$>99 \%$

$4.78 \quad 99.57$

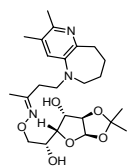

C24H37N3O6

463.2682

0.0082

93.4

$>99 \%$

$84.0 \%$

$95.6 \%$

$2.90 \quad 105.89$

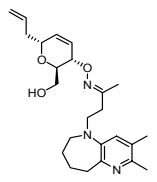

$\mathrm{C} 24 \mathrm{H} 35 \mathrm{~N} 3 \mathrm{O} 3$

413.2678

414

91.9

$>99 \%$

$>99 \%$

$>99 \%$

67.19

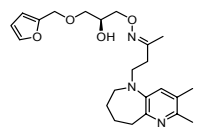

$\mathrm{C} 23 \mathrm{H} 33 \mathrm{~N} 3 \mathrm{O}$

415.2471

416

0.0076

96.6

$>99 \%$

$90.9 \%$

$>99 \%$

$4.40 \quad 80.33$ 


\begin{tabular}{|c|c|c|c|c|c|c|c|c|c|c|}
\hline STRUCTURE & Formula & Exact Mass & Mass Found & Mass/g & Yield \% & ELSD Purity & UV218 Purity & UV254 Purity & cLogP & TPSA \\
\hline & $\mathrm{C} 23 \mathrm{H} 34 \mathrm{~N} 6 \mathrm{O} 3$ & 442.2692 & 443.0 & 0.0082 & 97.8 & $>99 \%$ & $>99 \%$ & $>99 \%$ & 3.21 & 97.91 \\
\hline
\end{tabular}
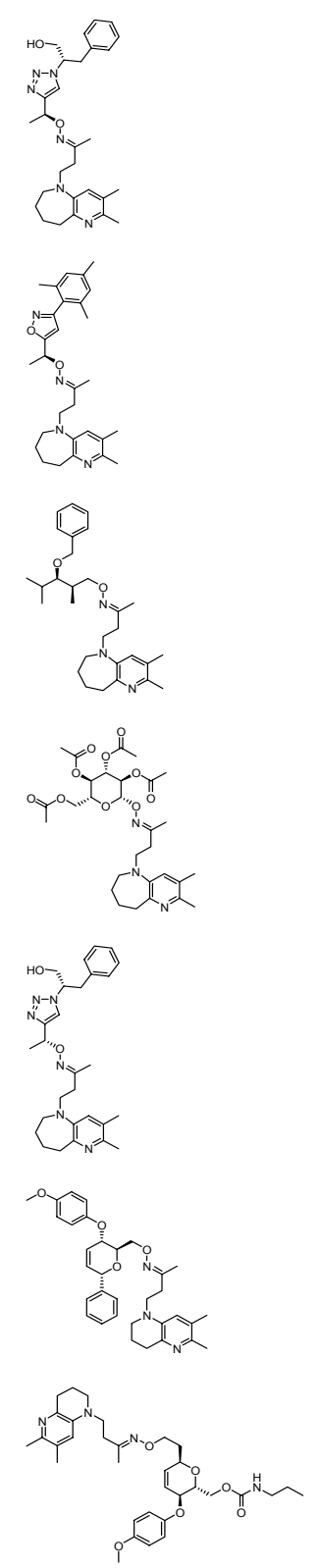

C33H46N4O6

594.3417

C28H38N6O2

490.3056

491.

0.0091

97.9

$>99 \%$

C33H39N3O4

541.2941

542.

0.0102

99.4

$97.6 \%$

$86.9 \%$

$93.4 \%$

$94.1 \%$

$96.1 \%$

3.56

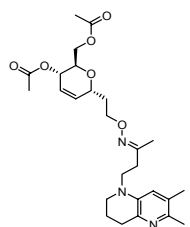

C26H37N3O6

487.2682

488

0.009

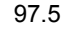

$97.7 \%$

N/A

$94.6 \%$

4.

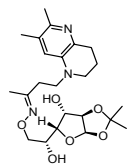

C23H35N3O6

449.2526

449

0.0078

91.6

$>99 \%$

N/A

$93.8 \%$

$2.2 \%$

$6.92 \quad 103.76$

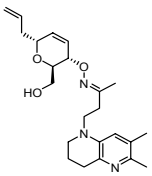

$\mathrm{C} 23 \mathrm{H} 33 \mathrm{~N} 3 \mathrm{O} 3$

399.2522
0.0072

95.1

$98.8 \%$
N/A

$93.5 \%$

4.59 
STRUCTURE

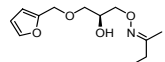

$\mathbb{N}^{n} \mathbb{N}^{N}$

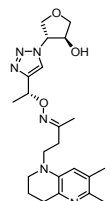

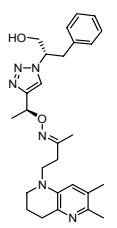

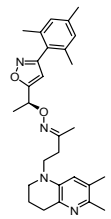

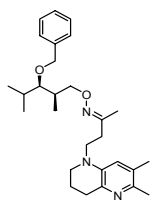

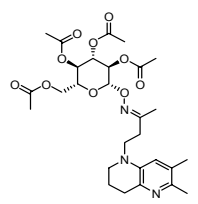

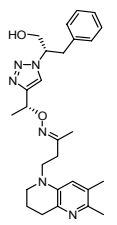

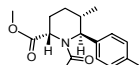

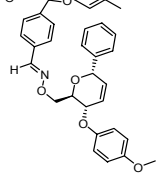

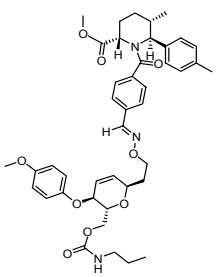

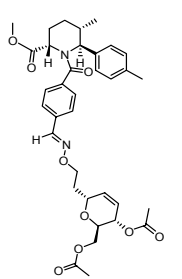

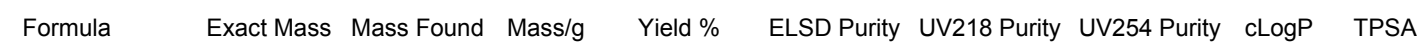
$\begin{array}{llllllllll}\mathrm{C} 22 \mathrm{H} 31 \mathrm{~N} 3 \mathrm{O} 4 & 401.2315 & 402.0 & 0.0074 & 97.3 & 98.5 \% & \mathrm{~N} / \mathrm{A} & 95.9 \% & 3.97 & 80.33\end{array}$ $\mathrm{C} 22 \mathrm{H} 32 \mathrm{~N} 6 \mathrm{O3}$

428.2536 429.0

0.0081

99.8

$>99 \%$

N/A

$96.8 \%$

$2.77 \quad 97.91$

$\mathrm{C} 27 \mathrm{H} 36 \mathrm{~N} 6 \mathrm{O} 2$

476.2900

477.1

0.0080

88.6

$98.9 \%$

N/A

$96.1 \%$

$5.02 \quad 88.67$

$\mathrm{C} 28 \mathrm{H} 36 \mathrm{~N} 4 \mathrm{O} 2$

460.2838

461.0

0.0086

98.6

$98.4 \%$

$85.6 \%$

$89.8 \%$

$7.49 \quad 63.76$

$\mathrm{C} 28 \mathrm{H} 41 \mathrm{~N} 3 \mathrm{O} 2$

451.3199

452.1

0.0082

95.9

$>99 \%$

N/A

$97.8 \%$

$6.93 \quad 46.96$

C28H39N3O

577.2635

578.

0.0100

91.4

$96.7 \%$

N/A

$90.4 \%$

$3.13 \quad 152.18$

$\mathrm{C} 27 \mathrm{H} 36 \mathrm{~N} 6 \mathrm{O} 2$

476.2900

477.0

0.0087

96.4

$>99 \%$

N/A

$97.1 \%$

$5.02 \quad 88.67$

$\mathrm{C} 42 \mathrm{H} 44 \mathrm{~N} 2 \mathrm{O} 7$

688.3149

689.3

0.0128

98.1

$>99 \%$

$98.3 \%$

$97.2 \%$

$8.38 \quad 95.91$

$\mathrm{C} 42 \mathrm{H} 51 \mathrm{~N} 3 \mathrm{O}$

741.3625

742.

0.013

$98.5 \%$

$90.7 \%$

$87.8 \%$

C35H42N2O9

634.2890

635.2

0.0120

99.8

$>99 \%$

$>99 \%$

$>99 \%$

$5.16 \quad 130.06$ 


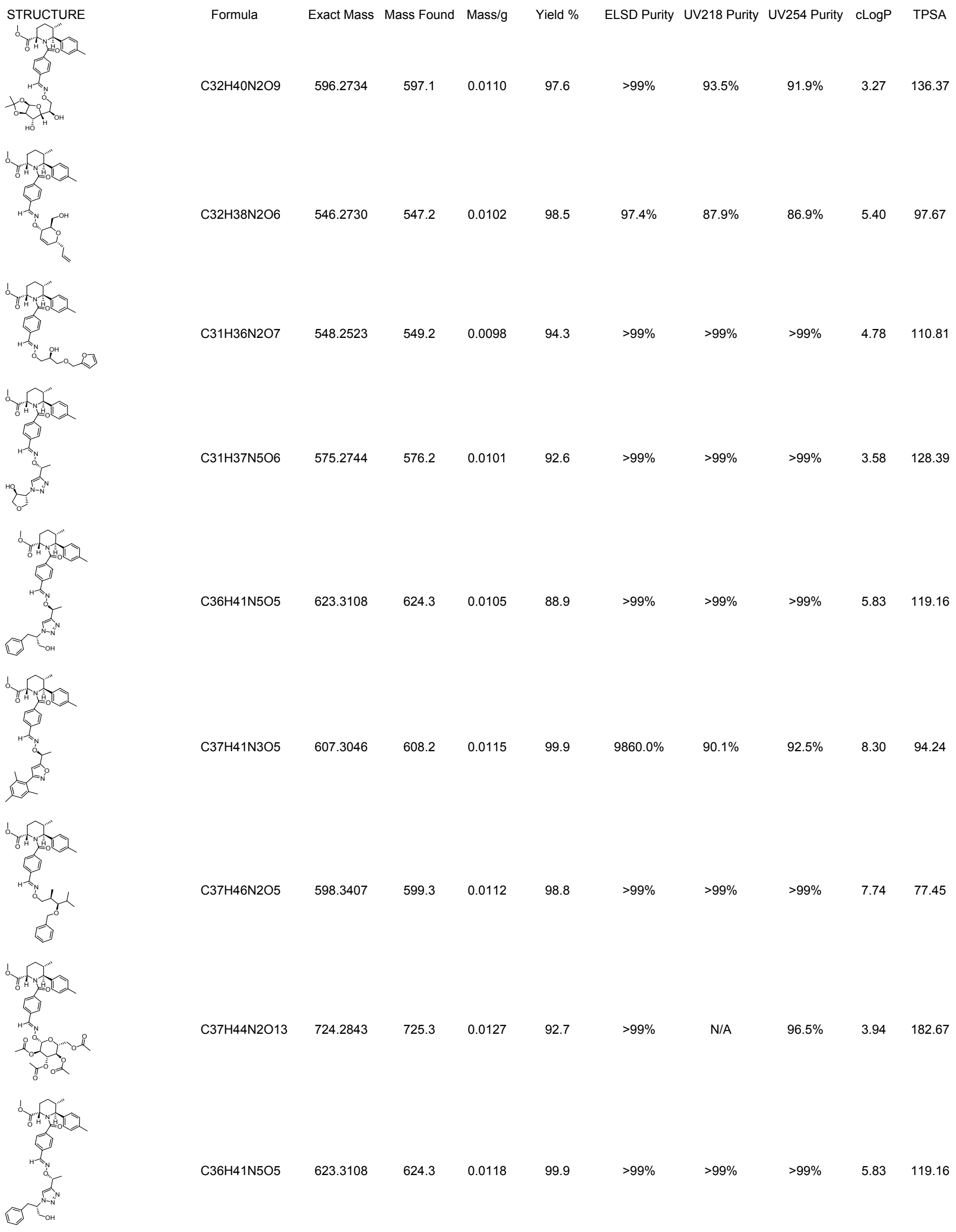




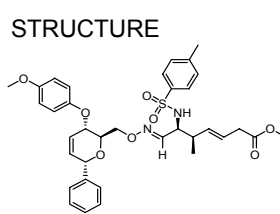

Formula

Exact Mass Mass Found Mass/g

Yield \%

C35H40N2O8S

648.2505

649

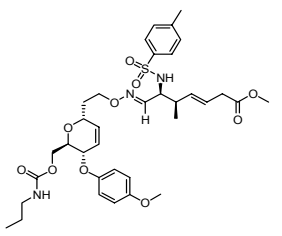

C35H47N3O10S

701.2982

702.3

0.0122

91.8

$>99 \%$

$>99 \%$

N/A

$5.46 \quad 160.11$

C28H38N2O10S

594.2247

595.1

0.0102

90.6

$>99 \%$

N/A

N/A

$2.89 \quad 155.92$

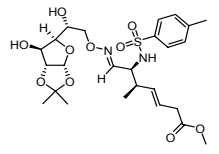

C25H36N2O10

556.2091

557

0.0073

69.2

$>99 \%$

$78.7 \%$

N/A

$1.00 \quad 162.23$
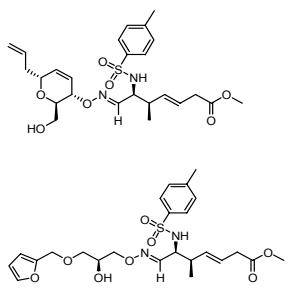

C24H32N2O8

508.1879

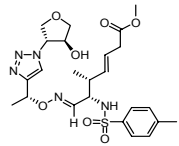

C24H33N5O7S

535.2101

536.0

0.0098

96.7

$>99 \%$

$>99 \%$

N/A

$1.31 \quad 154.25$

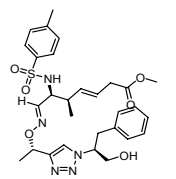

C29H37N5O6

583.2465

584

0.010
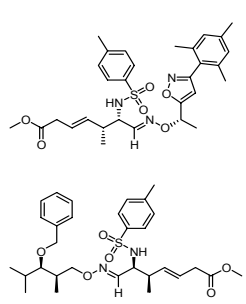

$\mathrm{C} 30 \mathrm{H} 42 \mathrm{~N} 2 \mathrm{O} 6 \mathrm{~S}$

558.2764

559

0.0102

96.4

$>99 \%$

$>99 \%$

$5.47 \quad 103.31$

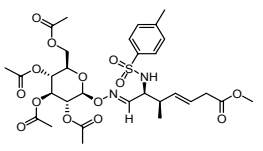

$\mathrm{C} 30 \mathrm{H} 40 \mathrm{~N} 2 \mathrm{O} 14$

684.2200

685.2

0.0128

98.7

$>99 \%$

N/A

N/A

$1.67 \quad 208.53$

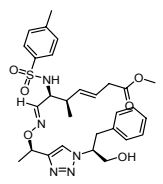

C29H37N5O6

583.2465

584.

0.0110

99.5

$>99 \%$

$92.5 \%$

N/A

$3.56 \quad 145.02$

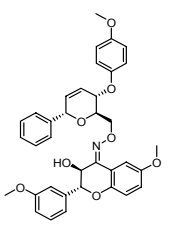

$\mathrm{C} 36 \mathrm{H} 35 \mathrm{NO} 8$

609.2363

610.1

0.0110

95.3

$>99 \%$

$94.2 \%$

$>99 \%$

$6.90 \quad 97.23$ 


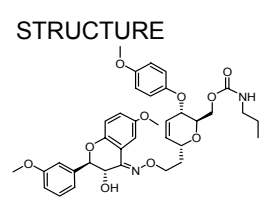

Formula Exact Mass Mass Found Mass/g Yield \% ELSD Purity UV218 Purity UV254 Purity cLogP TPSA

$\begin{array}{llllllllll}\mathrm{C} 36 \mathrm{H} 42 \mathrm{~N} 2 \mathrm{O} 10 & 662.2839 & 663.0 & 0.0122 & 97.2 & >99 \% & >99 \% & >99 \% & 6.25 & 135.56\end{array}$

$\underbrace{N_{0}}_{0}$

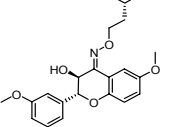

C29H33NO10

555.2104

555

0.0104

98.8

$>99 \%$

$97.5 \%$

$97.9 \%$

$3.68 \quad 131.37$

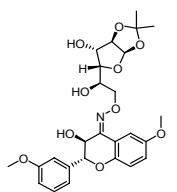

$\mathrm{C} 26 \mathrm{H} 31 \mathrm{NO} 10$

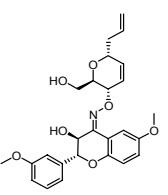

C26H29NO7

467.1944

$467.9 \quad 0.0087$

98.3

$>99 \%$

$>99 \%$

$>99 \%$

$3.92 \quad 98.99$

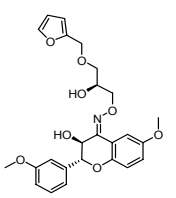

$\mathrm{C} 25 \mathrm{H} 27 \mathrm{NO} 8$

469.1737

469.

0.0086

96.7

$>99 \%$

$3.30 \quad 112.13$

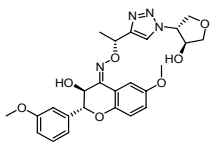

$\mathrm{C} 25 \mathrm{H} 28 \mathrm{~N} 4 \mathrm{O} 7$

496.1958

496

0.008

91.4

$>99 \%$

$>99 \%$

$98.3 \%$

$2.11 \quad 129.71$

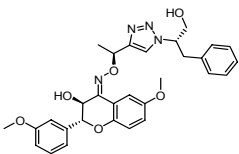

C3OH32N4

544.2322

545.

0.0101

97.9

$>99 \%$

$>99 \%$

$>99 \%$

$4.35 \quad 120.47$

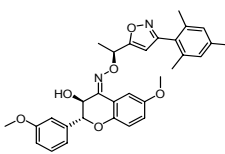

C31H32N2

528

0.0098

97.9

$97.2 \%$

$84.0 \%$

$90.1 \%$

$6.83 \quad 95.56$

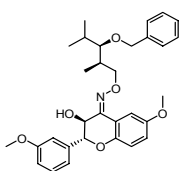

C31H37NO6

519.2621

520

0.0096

97.5

$>99 \%$

$96.1 \%$

$94.5 \%$

$6.26 \quad 78.76$

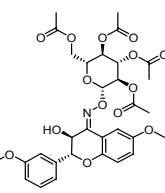

C31H35NO14

645.2058

646.1

0.0122

99.8

$96.6 \%$

$83.4 \%$

$89.1 \%$

$2.46 \quad 183.98$

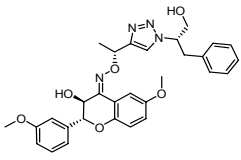

C3OH32N4

545.1

0.0097

94.0

$>99 \%$

$95.9 \%$

$>99 \%$

$4.35 \quad 120.47$

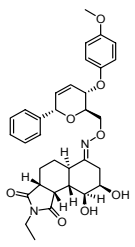

C33H38N2

$591.0 \quad 0.0071$

63.4

$87.8 \%$

$70.5 \%$

$68.3 \%$

$4.23 \quad 127.14$ 

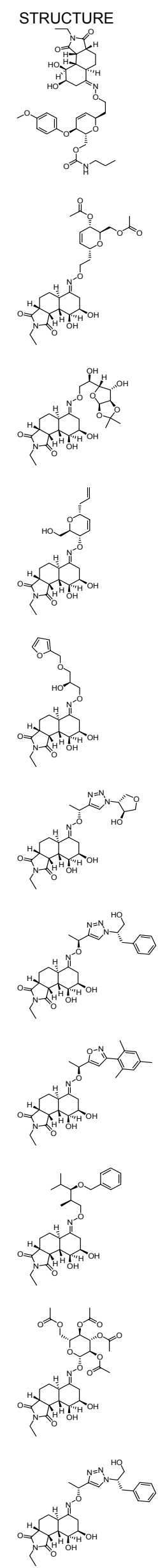

Formula

Exact Mass Mass Found Mass/g

Yield \%

ELSD Purity UV218 Purity UV254 Purity cLogP TPSA

C33H45N3O10

643.3105

644.

0.0078

63.6

$82.6 \%$

$64.4 \%$

$65.1 \%$

$3.58 \quad 165.47$

C26H36N2O10 536.2370

537

0.0101

99.4

$>99 \%$

$91.0 \%$

N/A

$0.79 \quad 161.28$

$\mathrm{C} 23 \mathrm{H} 34 \mathrm{~N} 2 \mathrm{O} 10$

498.2213

498

0.0085

90.0

$>99 \%$

$>99$

N/A

-1.09 167.59

$\mathrm{C} 23 \mathrm{H} 32 \mathrm{~N} 2 \mathrm{O} 7$

448.2210

448

90.6

$>99 \%$

$89.7 \%$

N/A

$1.03 \quad 128.90$

$\mathrm{C} 22 \mathrm{H} 30 \mathrm{~N} 2 \mathrm{O}$

450.200

50.2002

451.

0.0082

96.1

$>99 \%$

$>99$

N/A

$0.63 \quad 142.04$

C22H31N5

477.222

478.

0.0089

98.4

$>99 \%$

$92.6 \%$

N/A

$-0.56 \quad 159.61$

C27H35N5O6

526.

92.4

$>95 \%$

N/A

$1.69 \quad 150.38$

C28H35N3O

509.2526

510

0.0060

62.0

$96.9 \%$

$74.4 \%$

$68.9 \%$

$4.16 \quad 125.47$

C28H40N2O6

500.288

501.

97.0

$>99 \%$

$85.3 \%$

N/A

$3.59 \quad 108.67$

C28H38N2O

626.2323

627.0

0.0109

91.8

$>99 \%$

$>99 \%$

N/A

$-0.43 \quad 213.89$ 


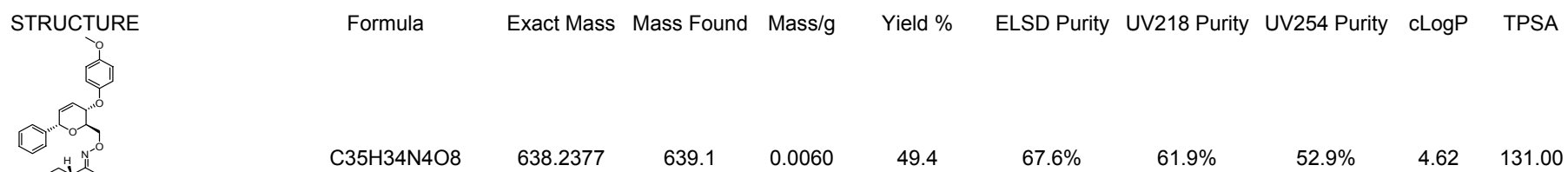

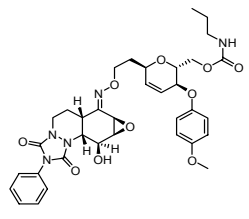

C35H41N5O10 691.2853

$\mathrm{C} 28 \mathrm{H} 32 \mathrm{~N} 4 \mathrm{O} 10$

584.2118
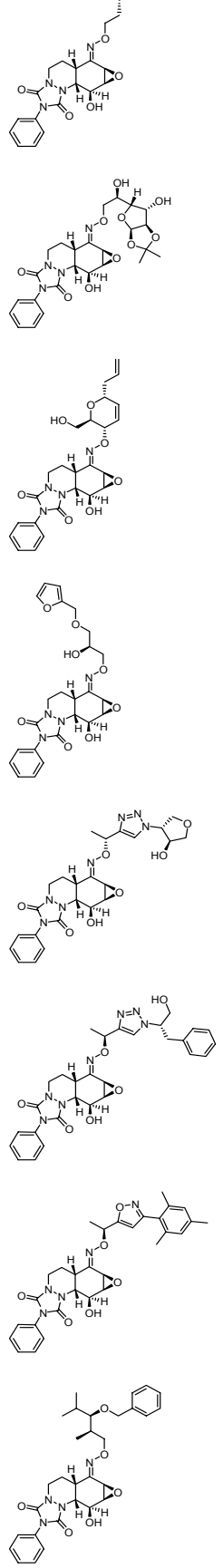

C25H30N4O10

$\mathrm{C} 25 \mathrm{H} 28 \mathrm{~N} 4 \mathrm{O} 7$

$\mathrm{C} 24 \mathrm{H} 26 \mathrm{~N} 4 \mathrm{O} 8$

498.1751

$\mathrm{C} 24 \mathrm{H} 27 \mathrm{~N} 7 \mathrm{O} 7$

C29H31N7O6

573.233

$\mathrm{C} 30 \mathrm{H} 31 \mathrm{~N} 5 \mathrm{O} 6$

$\mathrm{C} 30 \mathrm{H} 36 \mathrm{~N} 4 \mathrm{O} 6$
546.1962

546.

0.0097

93.7

$97.4 \%$

$89.0 \%$

N/A

$-0.49 \quad 171.46$

496.1958

497

0.0093

98.9

$97.9 \%$

$78.7 \%$

$91.6 \%$

(n)

499

99.6

525.1972

525

0.0089

89.4

$83.0 \%$

$58.3 \%$

$69.3 \%$

$-0.17 \quad 163.48$

574

0.0090

82.7

$90.6 \%$

$75.2 \%$

$82.8 \%$

2.0

154.24

557.2274

558

0.0066

62.9

$95.4 \%$

$84.2 \%$

$89.0 \%$

4.

548.2635

549.1

0.0099

95.3

$95.7 \%$

$92.0 \%$

$95.6 \%$

$3.98 \quad 112.53$ 

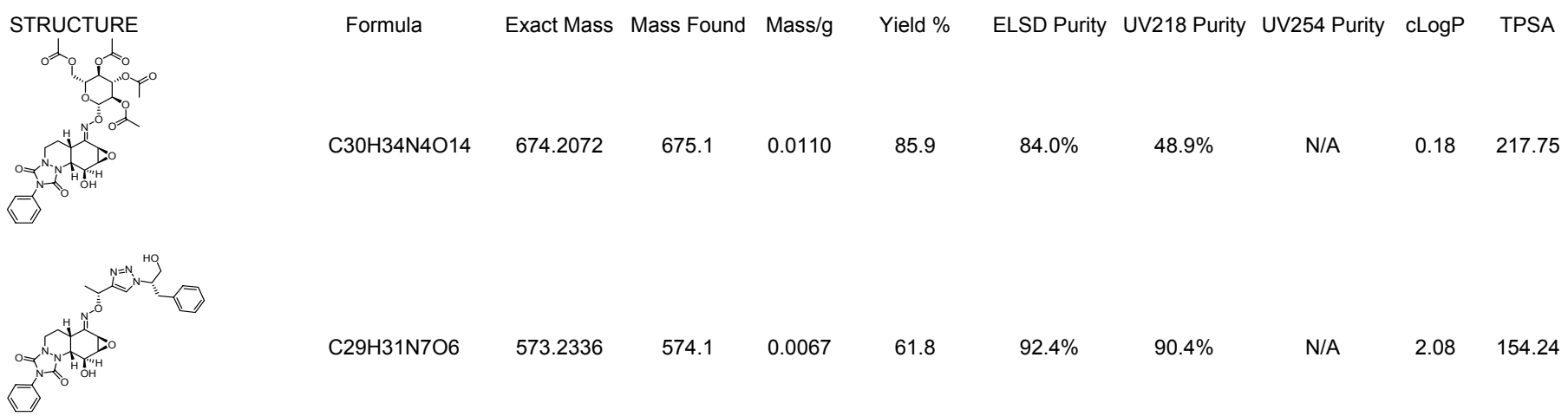

$\begin{array}{llllllllll}\mathrm{C} 29 \mathrm{H} 31 \mathrm{~N} 7 \mathrm{O} 6 & 573.2336 & 574.1 & 0.0067 & 61.8 & 92.4 \% & 90.4 \% & \mathrm{~N} / \mathrm{A} & 2.08 & 154.24\end{array}$

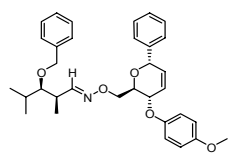

$\begin{array}{llllllllll}\mathrm{C} 33 \mathrm{H} 39 \mathrm{NO} 05 & 529.2828 & 530.1 & 0.0099 & 98.7 & >99 \% & 95.0 \% & 84.2 \% & 7.55 & 58.53\end{array}$

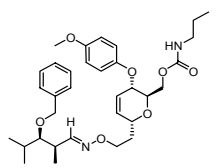

$\begin{array}{llllllllll}\mathrm{C} 33 \mathrm{H} 46 \mathrm{~N} 2 \mathrm{O} 7 & 582.3305 & 583.2 & 0.0101 & 91.5 & >99 \% & >99 \% & \mathrm{~N} / \mathrm{A} & 6.90 & 96.87\end{array}$

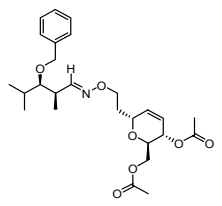

$\begin{array}{llllllllll}\mathrm{C} 26 \mathrm{H} 37 \mathrm{NO} & 475.2570 & 476.1 & 0.0084 & 93.3 & >99 \% & >99 \% & \mathrm{~N} / \mathrm{A} & 4.33 & 92.68\end{array}$

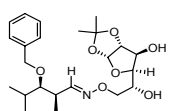

$\begin{array}{llllllllll}\mathrm{C} 23 \mathrm{H} 35 \mathrm{NO} 07 & 437.2414 & 438.0 & 0.0080 & 96.5 & >99 \% & 88.2 \% & \mathrm{~N} / \mathrm{A} & 2.44 & 98.99\end{array}$<smiles>CCC1CCC(CC(C)C(C)C(C)C)C1C(C)C</smiles>

$\mathrm{C} 23 \mathrm{H} 33 \mathrm{NO}$

387.2410

$\begin{array}{lll}387.9 & 0.0072 & 98.1\end{array}$

$>99 \%$

$>99 \%$

$\begin{array}{lll}\text { N/A } & 4.56 \quad 60.29\end{array}$

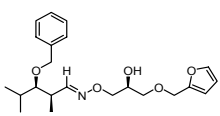

$\mathrm{C} 22 \mathrm{H} 31 \mathrm{NO}$

389.2202

390

0.007

94.9

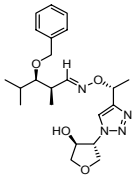

$\mathrm{C} 22 \mathrm{H} 32 \mathrm{~N} 4 \mathrm{O}$

417

0.0078

98.9

$>99 \%$

N/A

N/A

$2.75 \quad 91.01$

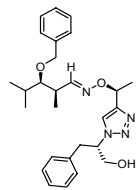

$\mathrm{C} 27 \mathrm{H} 36 \mathrm{~N} 4 \mathrm{O} 3$

464.2787

465.0

0.0088

100.0

$>99 \%$

$96.4 \%$

N/A

$5.00 \quad 81.78$

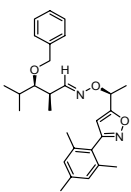

$\mathrm{C} 28 \mathrm{H} 36 \mathrm{~N} 2 \mathrm{O} 3$

448.2726

449.0

0.0080

94.2

$98.6 \%$

$94.1 \%$

$88.1 \%$

$7.47 \quad 56.86$

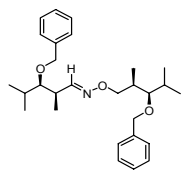

$\mathrm{C} 28 \mathrm{H} 41 \mathrm{NO}$

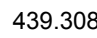

440

0.008

98.5

$95.0 \%$

N/A 


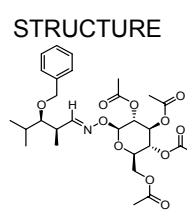

Formula

Exact Mass Mass Found Mass/g

Yield \%

ELSD Purity UV218 Purity UV254 Purity cLogP TPSA<smiles>CC(C)C(N=NC(N)(CO)Cc1ccccc1)C(C)C(Oc1ccccc1)C(C)C</smiles>

C28H39NO11

565.2523

566

95.2

$>99 \%$

$>99 \%$

N/A

$3.11 \quad 145.29$

$\mathrm{C} 27 \mathrm{H} 36 \mathrm{~N} 4 \mathrm{O} 3$

464.2787

465.1

0.0083

94.3

$>99 \%$

$>99 \%$

$\begin{array}{lll}\text { N/A } & 5.00 \quad 81.78\end{array}$<smiles>CCc1cc2c(nc1CC)CCN(CCC(C)=NOCc1ccc(-c3ccccc3)cc1)CC2Oc1ccc(OC)cc1</smiles>

C35H43N3

569.3254

570.

99.6

$>99 \%$

$91.3 \%$

N/A

$7.47 \quad 65.43$

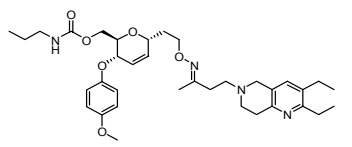

C35H50N4O6

622.3730

623.1

0.0118

99.9

$>99 \%$

$>99 \%$

N/A

$6.82 \quad 103.76$

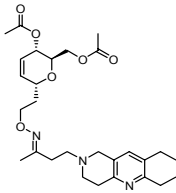

$\mathrm{C} 28 \mathrm{H} 41 \mathrm{~N} 3$

515.2995

516.

0.0093

95.2

$>99 \%$

N/A

$91.9 \%$

$4.25 \quad 99.57$

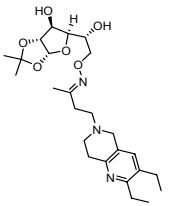

C25H39N3O

477.2839

477.

0.0089

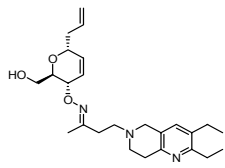

$\mathrm{C} 25 \mathrm{H} 37 \mathrm{~N} 3 \mathrm{O} 3$

427.2835

428.0

0.0081

100.0

$>99 \%$

N/A

N/A

$4.49 \quad 67.19$

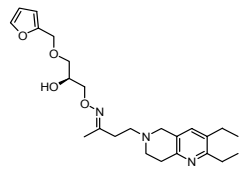

429.2628

430

0.0076

93.4

$>99 \%$

N/A

$>99 \%$

$3.87 \quad 80.33$

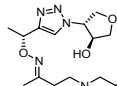

nis

C24H36N6

456.284

456

0.0080

92.

(n)

504.3213

505

0.009

99.4

$>99 \%$

N/A

$98.3 \%$

$4.93 \quad 88.67$

ind

篮

$\mathrm{C} 30 \mathrm{H} 40 \mathrm{~N} 4 \mathrm{O}$

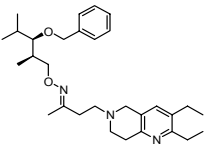

$\mathrm{C} 30 \mathrm{H} 45 \mathrm{~N} 3 \mathrm{O}$

479.3512

480.1

0.0083

91.4

$>99 \%$

N/A

$89.1 \%$

$6.83 \quad 46.96$

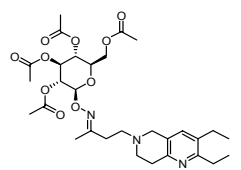

$\mathrm{C} 3 \mathrm{OH} 43 \mathrm{~N} 3 \mathrm{O} 10$

605.2948

606

$0.0113 \quad 98.5 \quad 95.3 \%$

N/A

$92.0 \%$

$3.03 \quad 152.18$ 


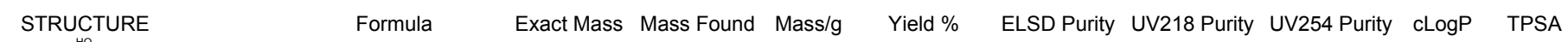

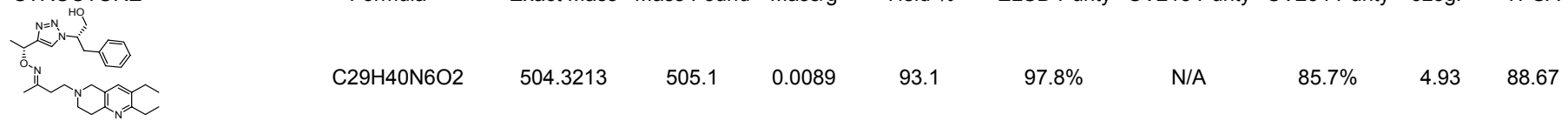

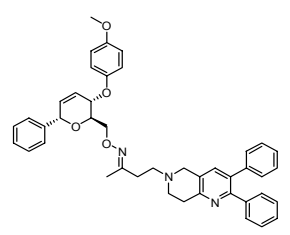

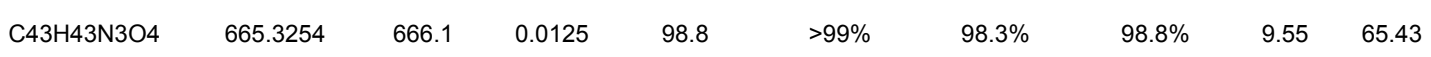

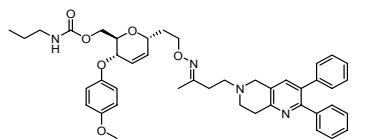

C43H50N4O6

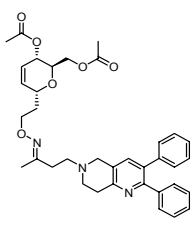

C36H41N3O6

611.2995

612

0.0108

92.8

$>99 \%$

$97.8 \%$

$>99 \%$

$6.33 \quad 99.57$

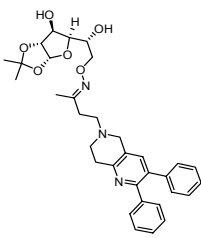

C33H39N3O

574

0.0100

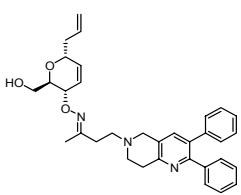

C33H37N3O3

523.2835

524

0.0088

88.3

$>99 \%$

$97.6 \%$

$97.6 \%$

$6.56 \quad 67.19$

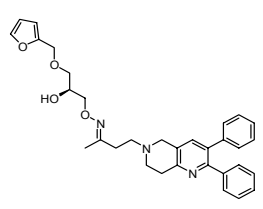

C32H35N3O4

525.2628

526.1

0.0089

89.4

$>99 \%$

$97.9 \%$

$>99 \%$

$5.95 \quad 80.33$

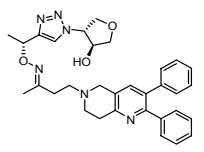

553.1

0.0099

94.2

$>99 \%$

$>99 \%$

$>99 \%$

$4.75 \quad 97.91$

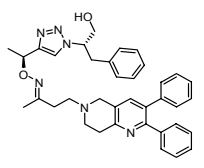

C37H40N6

600.3213

601.2

0.0108

94.9

$>99 \%$

$>99 \%$

$>99 \%$

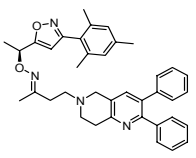

$\mathrm{C} 38 \mathrm{H} 40 \mathrm{~N} 4 \mathrm{O} 2$

584.3151

585.

97.5

$98.6 \%$

$92.0 \%$

$90.3 \%$

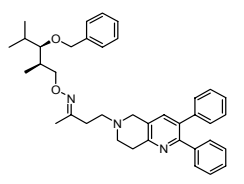

$\mathrm{C} 38 \mathrm{H} 45 \mathrm{~N} 3 \mathrm{O} 2$

575.3512

576.1

0.0109

99.9

$>99 \%$

$>99 \%$

$98.9 \%$

$8.91 \quad 46.96$

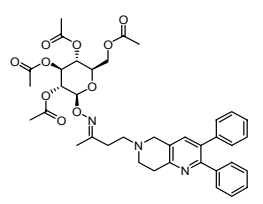

C38H43N3O10

701.2948

702.

0.0129

97.2

$97.3 \%$

$94.5 \%$

$95.0 \%$

$5.11 \quad 152.18$ 
STRUCTURE

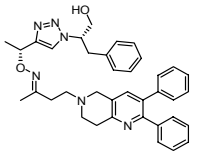

Formula

Exact Mass Mass Found Mass/g

Yield \%

ELSD Purity UV218 Purity UV254 Purity cLogP TPSA

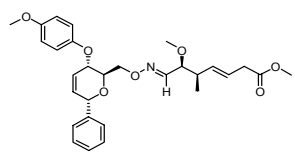

$\mathrm{C} 2 \mathrm{H} 42 \mathrm{~N} 2 \mathrm{O}$

562.2890

563.0

0.0105

98.5

$>99 \%$

$90.0 \%$

N/A

$4.84 \quad 123.17$

455.2155

455
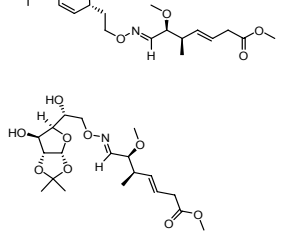

(1)

C19H29NO

367.1995

368

0.0064

92.0

$97.2 \%$

$>95 \%$

N/A

$2.28 \quad 86.60$

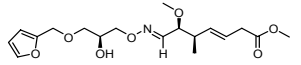

$\mathrm{C} 18 \mathrm{H} 27 \mathrm{NO}$

369.1788

369

0.0069

98.6

$98.4 \%$

$94.4 \%$

N/A

$1.88 \quad 99.74$

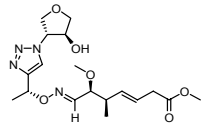

$\mathrm{C} 18 \mathrm{H} 28 \mathrm{~N} 4 \mathrm{O}$

(1)

C23H32N4O5

444.2373

444

0.0081

96.2

$>99 \%$

$94.4 \%$

N/A

$2.94 \quad 108.08$

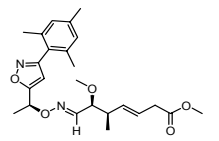

$\mathrm{C} 24 \mathrm{H} 32 \mathrm{~N} 2 \mathrm{O} 5$

428.2311

429.0

0.0077

94.9

$96.1 \%$

$89.1 \%$

$86.8 \%$

$5.41 \quad 83.17$

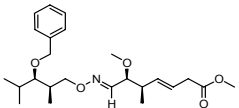

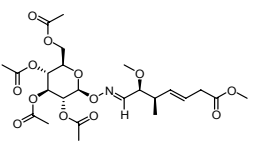

$\mathrm{C} 24 \mathrm{H} 35 \mathrm{NO} 13$

545.2108

546.0

0.0096

92.9

$98.9 \%$

N/A

N/A

$0.83 \quad 171.59$

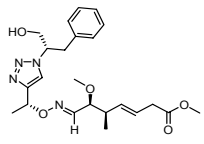

$\mathrm{C} 23 \mathrm{H} 32 \mathrm{~N} 4 \mathrm{O} 5$

444.2373
$0.0061 \quad 72.0$

$>99 \%$

$84.5 \%$

N/A

$2.94 \quad 108.08$

\section{9}

.

(2)

